\author{
UNIVERSIDADE DE SÃO PAULO \\ INSTITUTO DE FÍSICA DE SÃO CARLOS
}

THOMAS KAUÊ DAL'MASO PERON

Dynamics of Kuramoto oscillators in complex networks

São Carlos 



\section{Dynamics of Kuramoto oscillators in complex networks}

Thesis submitted to the Physics Graduate Program at the São Carlos Institute of Physics, University of São Paulo, to obtain the degree of Doctor of Sciences.

Concentration area: Applied Physics Option: Computational Physics Advisor: Prof. Dr. Francisco Aparecido Rodrigues

Corrected version

(Original version available on the Program Unit)

São Carlos 
I AUTHORIZE THE REPRODUCTION AND DISSEMINATION OF TOTAL OR PARTIAL COPIES OF THIS DOCUMENT, BY CONVENCIONAL OR ELECTRONIC MEDIA FOR STUDY OR RESEARCH PURPOSE, SINCE IT IS REFERENCED.

Cataloguing data revised by the Library and Information Service of the IFSC, with information provided by the author

Peron, Thomas Kauê Dal'Maso

Dynamics of Kuramoto oscillators in complex networks / Thomas Kauê Dal'Maso Peron; advisor Francisco Aparecido Rodrigues - revised version -São Carlos 2017.

$148 \mathrm{p}$.

Thesis (Doctorate - Graduate Program in Computational Physics) -- Instituto de Física de São Carlos, Universidade de São Paulo - Brasil, 2017.

1. Complex networks. 2. Synchronization. 3. Kuramoto model. 4. Nonlinear dynamics. I. Rodrigues, Francisco Aparecido, advisor. II. Title. 


\section{Acknowledgements}

I would like to thank Prof. Francisco Rodrigues for supervising this PhD and for offering me all the scientific opportunities that I had in this project, and in particular for giving me the freedom to explore my ideas. I am very grateful to Prof. Luciano da Fontoura Costa for hosting me in his group during the last six years and for the many thoughtprovoking discussions about science and music in the weekly rehearsals. My deepest gratitude to Prof. Jürgen Kurths for welcoming me multiple times in the Potsdam-Institut für Klimafolgenforschung (PIK). I cannot put into words how greatly I have benefited from his collaboration and all the experience of working with his group in the wonderful campus of Telegrafenberg in Potsdam. I also thank Prof. Lutz Schimansky-Geier for his invaluable comments and for guiding our research on stochastic oscillators.

Throughout the course of my PhD I have had the opportunity to work with amazing collaborators. This thesis is definitely a result of intense joint work with them. In particular, I am thankful to Peng Ji for starting a random chat about Kuramoto oscillators during a lunch time near the Pappelallee campus. That conversation evolved to very productive four years of collaborative research along with many insightful and funny discussions. I want to thank Deniz Eroglu for being such an amazing friend; life in Potsdam would certainly be a lot less fun without him. I also need to thank him for giving me the opportunity to see what a beautiful country Turkey is and for the memorable time there. Moreover, his comments to this thesis are very much appreciated. I consider myself very lucky to have met and worked with Bernard Sonnenschein, whose friendship and collaboration in these years I am very grateful for. I cannot describe how much I have learned through our stimulating and almost continuous Skype talks. Many thanks to Paul Schultz for all his help in Potsdam and for allowing me to show my (aero)drumming skills with PIK Band.

I feel very grateful to many other people I had the chance to meet in PIK and in Humboldt-Universität zu Berlin, specially Stefan Ruschel, Ozgur Afsar, Tim Kittel, Lyuba Tupikina, Justus Kromer, David Hansmann and Carsten Grabow.

I would also like to thank my brazilian collaborators Cesar Comin, Filipi Nascimento, Guilherme Ferraz de Arruda and Diego Amancio; and my officemates Cynthia Couto and Henrique Ferraz de Arruda for creating a very pleasant work atmosphere in the São Carlos Institute of Physics. Further thanks to Edmilson Roque dos Santos for the many discussions on coupled oscillators and life in general; and to Vinicius Sciuti for the assistance with local issues while I was abroad. I also want to thank Lucas T. Assirati for reading and giving comments on parts of this thesis. 
I am deeply thankful to my mother and first teacher Eunice Maria Dal'Maso for all her confidence and efforts to bring me where I am today.

Finally, my profound thanks to Alessandra for her support, encouragement, patience, understanding and for kindly tolerating the months apart.

I acknowledge FAPESP for the financial support under the grants 2012/22160-7 and 2015/02486-3 (BEPE scholarship). 
"The good student, then, ought to be humble and docile, free alike from vain cares and from sensual indulgences, diligent and zealous to learn willingly from all, to presume never upon his own knowledge, to shun the authors of perverse doctrine as if they were poison, to consider a matter thoroughly and at length before judging of it, to seek to be learned rather than merely to seem so, to love such words of the wise as he has grasped, and ever to hold those words before his gaze as the very mirror of his countenance. And if some things, by chance rather obscure, have not allowed him to understand them, let him not at once break out in angry condemnation and think that nothing is good but what he himself can understand. This is the humility proper to a student's discipline." (Hugh of St. Victor, Didascalicon, B. 3, Ch. 13) 



\section{Abstract}

PERON, T.K.DM. Dynamics of Kuramoto oscillators in complex networks. 2017. 148 p. Tese (Doutorado em Ciências) - Instituto de Física de São Carlos, Universidade de São Paulo, São Carlos, 2017.

Synchronization of an ensemble of oscillators is an emergent phenomenon present in several complex systems, ranging from biological and physical to social and technological systems. The most successful approach to describe how coherent behavior emerges in these complex systems is given by the paradigmatic Kuramoto model. For decades, this model has been traditionally studied in globally coupled topologies. However, besides being intrinsically dynamical, complex systems exhibit very heterogeneous structure, which can be represented as complex networks. This thesis is dedicated to the investigation of fundamental problems regarding the collective dynamics of Kuramoto oscillators coupled in complex networks. First, we address the effects on network dynamics caused by the presence of triangles, which are structural patterns that permeate real-world networks but are absent in random models. By extending the heterogeneous degree mean-field approach to a class of configuration model that generates random networks with variable clustering, we show that triangles weakly affect the onset of synchronization. Our results suggest that, at least in the low clustering regime, the dynamics of clustered networks are accurately described by tree-based theories. Secondly, we analyze the influence of inertia in the phases' evolutions. More precisely, we substantially extend the mean-field calculations to second-order Kuramoto oscillators in uncorrelated networks. Thereby hysteretic transitions of the order parameter are predicted with good agreement with simulations. Effects of degree-degree correlations are also numerically scrutinized. In particular, we find an interesting dynamical equivalence between variations in assortativity and damping coefficients. Potential implications to real-world applications are discussed. Finally, we tackle the problem of two intertwined populations of stochastic oscillators subjected to asymmetric attractive and repulsive couplings. By employing the Gaussian approximation technique we derive a reduced set of ODEs whereby a thorough bifurcation analysis is performed revealing a rich phase diagram. Precisely, besides incoherence and partial synchronization, peculiar states are uncovered in which two clusters of oscillators emerge. If the phase lag between these clusters lies between zero and $\pi$, a spontaneous drift different from the natural rhythm of oscillation emerges. Similar dynamical patterns are found in chaotic oscillators under analogous couplings schemes.

Keywords: Complex networks. Synchronization. Kuramoto model. Nonlinear dynamics. 



\section{Resumo}

PERON, T.K.DM. Dinâmica de osciladores de Kuramoto em redes complexas. 2017. 148 p. Tese (Doutorado em Ciências) - Instituto de Física de São Carlos, Universidade de São Paulo, São Carlos, 2017.

Sincronização de conjuntos de osciladores é um fenômeno emergente que permeia sistemas complexos de diversas naturezas, como por exemplo, sistemas biológicos, físicos, naturais e tecnológicos. A abordagem mais bem sucedida na descrição da emergência de comportamento coletivo em sistemas complexos é fornecida pelo modelo de Kuramoto. Durante décadas, este modelo foi tradicionalmente estudado em topologias completamente conectadas. Entretanto, além de ser intrinsecamente dinâmicos, tais sistemas complexos possuem uma estrutura altamente heterogênea que pode ser apropriadamente representada por redes complexas. Esta tese é dedicada à investigação de problemas fundamentais da dinâmica coletiva de osciladores de Kuramoto acoplados em redes. Primeiramente, abordamos os efeitos sobre a dinâmica das redes causados pela presença de triângulos - padrões que estão omnipresentes em redes reais mas estão ausentes em redes gerados por modelos aleatórios. Estendemos a abordagem via campo-médio para uma variação do modelo de configuração tradicional capaz de criar topologias com número variável de triângulos. Através desta abordagem, mostramos que tais padrões estruturais pouco influenciam a emergência de comportamento coletivo em redes, podendo a dinâmica destas ser descrita em termos de teorias desenvolvidas para redes com topologia local semelhante a grafos de tipo árvore. Em seguida, analisamos a influência de inércia na evolução das fases. Mais precisamente, generalizamos cálculos de campo-médio para osciladores de segunda-ordem acoplados em redes sem correlação de grau. Demonstramos que na presença de efeitos inerciais o parâmetro de ordem do sistema se comporta de forma histerética. Ademais, efeitos oriundos de correlações de grau são examinados. Em particular, verificamos uma interessante equivalência dinâmica entre variações nos coeficientes de assortatividade e amortecimento dos osciladores. Possíveis aplicações para situações reais são discutidas. Finalmente, abordamos o problema de duas populações de osciladores estocásticos sob a influência de acoplamentos atrativos e repulsivos. Através da aplicação da aproximação Gaussiana, derivamos um conjunto reduzido de EDOs através do qual as bifurcações do sistema foram analisadas. Além dos estados asíncrono e síncrono, verificamos a existência de padrões peculiares na dinâmica de tal sistema. Mais precisamente, observamos a formação de estados caracterizados pelo surgimento de dois aglomerados de osciladores. Caso a defasagem entre estes grupos é inferior a $\pi$, um novo ritmo de oscilação diferente da frequência natural dos vértices emerge. Comportamentos dinâmicos similares são observados em osciladores caóticos sujeitos a acoplamentos análogos. 
Palavras-chave: Redes Complexas. Sincronização. Modelo de Kuramoto. Dinâmica nãolinear. 


\section{List of Figures}

Figure 1 - Typical dependence of the order parameter $R$ as function of the coupling $\lambda$ for a population of first-order Kuramoto oscillators coupled in the fully connected graph considering $g(\omega)=\left[\pi\left(\omega^{2}+1\right)\right]^{-1}$. Dots are obtained by numerically evolving Eqs. 2.12 with $N=10^{4}$ oscillators. Solid line is derived by solving Eq. 2.19. . . . . . . . . . . . . . . . . 41

Figure 2 - (a) Illustration of a damped pendulum of mass $m$ subjected to a constant torque $\omega$. (b) Schematic diagram of $\omega \times\langle\dot{\theta}\rangle$. . . . . . . . . . . 47

Figure 3 - Stability diagram of Eq. $2.47 \ldots \ldots$. . . . . . . . . . 49

Figure 4 - Hysteretic synchrony of the second-order Kuramoto model. Critical conditions (Eq. 2.55) for the backward propagation of the system are depicted in the plot. Dashed lines are used to highlight the critical point $\left(\lambda_{c}^{\text {low }}, R_{c}^{\mathrm{II}}\right)$ predicted by the theory. Theoretical solutions are derived by solving Eqs. 2.50-2.53. Dots are obtained by numerically evolving Eqs. 2.39 considering $N=10^{3}$ oscillators, $\alpha=1, m=1, \Omega_{\mathrm{M}}=5$ and $d t=0.01$. Order parameter $R$ is averaged over the time span $t \in[200,500]$.

Figure 5 - Example of a node attached to three single edges and two triangles with $k_{i}=s_{i}+2 t_{\triangle i}=7$. Therefore, the $i$ th element of the joint degree sequence is $\{3,2\} \ldots \ldots \ldots \ldots \ldots 6$

Figure 6 - Synchronization diagram for networks constructed with the configuration model for clustered networks considering (a) $P\left(s, t_{\triangle}\right)$ as a double Poisson degree distribution (Eq. 3.12) and (b) double SF degree distribution $P\left(s, t_{\triangle}\right) \propto s^{-\gamma} t_{\triangle}^{-\gamma}$, where $\gamma=3$. Lines are obtained by numerically solving Eq. 3.10 for different values of $\lambda$ and dots are obtained numerically evolving Eqs. 2.23. Each point is an average over 10 different realizations. Other parameters: $N=10^{3}$ and $\langle k\rangle=20 . \quad$. . . 58

Figure 7 - Synchronization diagrams calculated as in Fig. 6 for random networks with degree distribution (a) $P(k)=e^{-\langle k\rangle}\langle k\rangle^{k} / k$ !, (b) $P(k) \propto k^{-\gamma}$, where $\gamma=3$; and joint degree distribution generated by Eq. 3.14. Each point is an average over ten network realizations. Solid lines correspond to the theoretical prediction from Eq. 3.10. . . . . . . . . . . . . . 59

Figure 8 - Synchronization diagrams calculated as in the other figures for random networks with degree distribution (a) $P(k)=e^{-\langle k\rangle}\langle k\rangle^{k} / k$ !, (b) $P(k) \propto$ $k^{-\gamma}$ with $\gamma=3$, and joint degree distribution generated by Eq. 3.14 with $f=1$. Each point is an average over ten network realizations. Solid lines correspond to the theoretical prediction from Eq. 3.10. . . . 
Figure 9 - Parameter space of Eq. 4.16 for when the coupling is (a) increased and (b) decreased. The red area represents the region with the stable fixed point solution, whereas the gray area indicates the parameter combinations that yield stable limit cycles. The triangles show the evolution in the parameter space of oscillators with degree $k=8$ and 20, starting in incoherence in (a) [coherence in (b)] and approach the synchronous state (incoherence) as the coupling is increased (decreased). Parameters: $\alpha=0.1, D=0.1,\langle k\rangle=10, N=3000$, and $P(k) \propto k^{-\gamma}$, with $\gamma=3 \ldots \ldots \ldots \ldots \ldots \ldots \ldots$

Figure 10 - (a) Analytical and numerical curves of the order parameter $r$ and (b) evolution of $C(\lambda r)$ for the case in which the coupling strength is increased. For the analytical curve, we set $C(\lambda r)=0$ for $\lambda<\lambda_{c}^{\mathrm{I}}$. In both panels the theoretical results are derived from Eqs. 4.8, 4.13, 4.15 and 4.18 using the range of synchronous degrees for increasing (Eq. 4.18) and decreasing (Eq. 4.20) coupling strength. Parameters of the model: $\alpha=0.1, D=0.1$. Simulation parameters: SF network with $N=3000$ nodes, $\langle k\rangle=10, k_{\min }=5$ and degree distribution $P(k) \sim k^{-\gamma}$, with $\gamma=3$.

Figure 11 - (a) Average frequency $\langle\omega\rangle_{k}$ of oscillators with same degree $k$ and (b) range of degrees of synchronous nodes. The simulation parameters are the same as in Fig. 10. . . . . . . . . . . . . . . . . 70

Figure 12 - Synchronization diagrams for networks with degree distribution $P(k) \sim$ $k^{-\gamma}$ for different exponent $\gamma$. Lower (upper) curves correspond to the forward (backward) propagation of the coupling strength. The analytical plots are calculated from the summation of Eqs. 4.22 and 4.24. All networks have $N=3000$ and $\langle k\rangle=10 \ldots \ldots$. . . . . . . . 71

Figure 13 - Synchronization diagrams for different values of the disorder parameter $q$. Analytical results are obtained from the summation of $r_{\text {lock }}^{\mathrm{q}}$ and $r_{\mathrm{drift}}^{\mathrm{q}}$. The same network topology used in Fig. 2 was used in the simulations of all panels in this figure. Lower (upper) curves correspond to the forward (backward) propagation of the coupling strength. Other parameters: $N=3000,\langle k\rangle=10, \alpha=0.1, D=0.1$ and distribution of quenched disorder as $g(\varepsilon)=1 / 2 \varepsilon$ for $\varepsilon \in[-q, q]$. . . . . . . . . . . . .

Figure 14 - Synchronization diagram $r(\lambda)$ for (a) $\mathcal{A}=-0.3$, (b) $\mathcal{A}=-0.1$, (c) $\mathcal{A}=0.1$ and $(\mathrm{d}) \mathcal{A}=0.3$. The dissipation coefficient is fixed at $\alpha=1$ with natural frequencies given by $\Omega_{i}=k_{i}-\langle k\rangle$, as in Fig. 15. All networks considered have $N=10^{3},\langle k\rangle=6$ and $P(k) \sim k^{-\gamma}$, where $\gamma=3$. 
Figure 15 - Synchronization diagram $r(\lambda)$ with (a) $\alpha=0.2$, (b) $\alpha=0.4$, (c) $\alpha=0.6$ and (d) $\alpha=0.8$ for disassortativity $\mathcal{A}=-0.3$. With increasing $\alpha$, onset of synchronization and hysteresis decreases. The natural frequency of each oscillator is $\Omega_{i}=k_{i}-\langle k\rangle$ and the networks have $N=10^{3}$ and $\langle k\rangle=6$. The degree distribution follows a power-law $P(k) \sim k^{-\gamma}$, where $\gamma=3$. Curves in which points are connected by solid lines (resp. dashed lines) correspond to the forward (resp. backward) continuations of the coupling strength $\lambda$.

Figure 16 - Contour plot on $\alpha-\mathcal{A}$ plane colored according to (a) the maximal order parameter $\mathcal{M O}$, (b) the maximal order parameter difference $\mathcal{M D}$ and (c) the hysteresis area $\mathcal{S}$. $\mathcal{A}$ and $\alpha$ are varied within the interval $[-0.3,0.3]$ and $[0.2,1]$, respectively. For each pair $(\alpha, \mathcal{A}), 40$ times simulations are performed with the coupling strength in the interval as in Fig. 15, i.e $\lambda \in[0,25]$.

Figure 17 - Parameters are the same as in Fig. 15, except that the natural frequencies are randomly selected from a Lorentzian distribution $g(\Omega)=$ $1 /\left(\pi\left(1+\Omega^{2}\right)\right) \ldots \ldots \ldots \ldots \ldots \ldots \ldots$

Figure 18 - Parameters are the same as in Fig. 14, except that natural frequencies are randomly selected from a Lorentzian distribution $g(\Omega)=1 /(\pi(1+$ $\left.\Omega^{2}\right)$. . . . . . . . . . . . . . . . .

Figure 19 - Contour plots similar as in Fig. 16 for networks with a natural frequency distribution given by $g(\Omega)=1 /\left(\pi\left(1+\Omega^{2}\right)\right)$. . . . . . . . . . . .

Figure 20 - Schematic visualization of the asymmetric interactions between subpopulations. . . . . . . . . . . . . . . . . . . 90

Figure 21 - Snapshots of oscillators' phases. (a) $\pi$-state with $K_{0}=0, G_{0}=0$, $\Delta K=8, \Delta G=2$ yielding $r_{1} \simeq 0.92, r_{2} \simeq 0.92,|\delta| \simeq \pi, r \simeq 0$ and $|\Omega| \simeq 0$. (b) Traveling wave state at $K_{0}=1, G_{0}=18, \Delta K=8$ and $\Delta G=2$ leading to $r_{1} \simeq 0.71, r_{2} \simeq 0.98,|\delta| \simeq 0.77 \pi$ and $|\Omega| \simeq 14 . \quad . \quad 92$

Figure 22 - Bifurcation diagrams for (a) $K_{0}=2, G_{0}=3$ and (b) $\Delta K=8, \Delta G=2$. Solid lines are derived from Eqs. 5.36 and 5.37, dashed lines obtained with MATCONT (168). "Zero-lag sync" denotes partially synchronized states with zero phase lag between the two subpopulations. Parameter regions in which $\delta=\pi$ are labeled as " $\pi$-states". Traveling wave states (TW1 and TW2) are the regions in the parameter space for which the oscillators exhibit non-zero values for the wave speed $\Omega$. (c) Zooms showing coexistence of traveling waves with other collective states. Red dotted lines (plotted only here) obtained with MATCONT. . . . . . . . . . 94 
Figure 23 - Order parameters $r_{1}, r_{2}, r$ and phase lag $|\delta|$ as a function of average in-coupling strength $K_{0}$ for (a) $G_{0}=0$ and (b) $G_{0}=2$. Remaining parameters in both cases: $\Delta K=8$ and $\Delta G=2$. (c) $\Delta K=3, \Delta G=10$ and $G_{0}=2$. In all panels, dots are obtained by numerically evolving Eqs. 5.1 with $N=10^{4}$ oscillators. Solid lines are obtained by numerically solving Eqs. 5.31, 5.32 and 5.32. In order to detect the hysteresis in (b), forward $(\triangleright)$ and backward propagations $(\triangleleft)$ are performed. Shaded areas are colored according to the respective regions in the parameter spaces of Fig. 22. . . . . . . . . . . . . . . . . . . . . . . . . 96

Figure 24 - Comparison between simulations (colormaps) and theory (lines). (a) and (b) depict the local order parameters $r_{2}$ and $r_{1}$, respectively; and (c) shows the global order parameter $r$. (d) Absolute value of the wave speed $|\Omega|$. Inset shows zoomed area with rescaled colormap. Simulations in panels (a)-(d) were performed with initial conditions were randomly chosen from uniform distribution defined in $[-\pi, \pi]$. In panels (e)-(g) simulations are repeated with initial conditions as $\theta_{i}(0)=1 \forall i$. Remaining parameters are the same as in Fig. 22(b). . . . . . . . . . . 98

Figure 25 - Spontaneous drift measured by the the locking frequency $\left|\langle\dot{\psi}\rangle_{t}\right|$, Eq. 5.34 (theory [lines] vs. simulation [dots]) and wave speed $|\Omega|$, Eq. 5.30 only simulation) . . . . . . . . . . . . . . . . . . . . 99

Figure 26 - (a) Order parameters $r_{1}, r_{2}$ and $r$; and (b) phase lag $\delta$ for a random binary network with degrees $k_{1}=10$ and $k_{2}=15$. Remaining parameters: $N=1000, \Delta K=12, \Delta G=2, G_{0}=3$ and $D=0.5 \ldots \ldots 2$

Figure 27 - Phase portrait of the dynamics of the Rössler system (Eq. 5.46) with (a) phase-coherent attractor $(a=0.15, b=0.4, c=8.5)$ and (b) funnel attractor $(a=0.2, b=0.4, c=7.5) \ldots \ldots \ldots 104$

Figure 28 - Snapshots of the oscillators' trajectories of the weighted $x$-coupled Rössler model [Eq. 5.51 with phase-coherent attractor $(a=0.15, b=$ 0.2 , and $c=10$ ). (a) $\pi$-state where $\delta=\pi$ with $K_{0}=0$ and $G_{0}=0$. (b) Lag sync state for which $\delta<\pi$ with $K_{0}=0$ and $G_{0}=0.2$. Gray line in the background corresponds to a trajectory of a randomly selected oscillator in order to depict typical dynamics. Remaining parameters: $N=200, \Delta K=0.1, \Delta G=0.1$, and $D=0.03$. See the supplemental material of (161) for the animated version of the figure. . . . . . . . 106 
Figure 29 - (a) Map showing the regions with escaping trajectories for weighted $x$-coupled Rössler oscillators (Eq. 5.51) in the phase-coherent regime considering $N=500, D=0, \Delta K=0.1$, and $\Delta G=1$. For each pair $\left(G_{0}, K_{0}\right)$ the initial conditions $\left[x_{i}(0), y_{i}(0), z_{i}(0)\right] \forall i$ are randomly drawn according to a uniform distribution in the range $[-1,1]$. Simulation time $T=1 \times 10^{5}$. Solid black curves fitting the boundaries of the escaping region are obtained through the relation $\langle\langle K G\rangle\rangle=c$, where $c_{\mathrm{I}}=c_{\mathrm{III}}=$ 11.8 and $c_{\mathrm{II}}=c_{\mathrm{IV}}=-0.66$. Square at the center depicts the parameter region of Fig. 30. Area of escaping region as function of (b) simulation time $T$ for fixed $D=0$ and (c) as function of $D$ for $T=5 \times 10^{3} \ldots 108$

Figure 30 - Colormaps showing (a) order parameter $r$, (b) phase lag $\delta$ and (c) speed of phase wave $\left|\Omega-\omega_{0}\right|$ for weighted $x$-coupled Rössler oscillators (Eq. 5.51) with phase-coherent (upper triangles) and funnel (lower triangles) attractors considering $D=0.03$. (d) Area of TW state in which $\left|\Omega-\omega_{0}\right| \geq \varepsilon$, where we consider $\varepsilon=0.3$; and $\pi$-state for $\delta \geq \varepsilon$ with $\varepsilon=3$. Remaining parameters: $\Delta K=0.1, \Delta G=1, N=10^{3}, 50 \times 50$ grid, simulation time $T=5 \times 10^{3}$. Curves in the interface between incoherent and partial synchronized state for phase-coherent oscillators are given by the condition $2 D_{\text {eff }}=\langle\langle K G\rangle\rangle$, here with $D_{\text {eff }} \approx 0$. Horizontal dashed lines correspond to the critical points $K_{0 c}= \pm \Delta K / 2$. See also Appendix B for diagrams with $\Delta K=\Delta G=0 \ldots \ldots$. . . . . . . 109

Figure 31 - Colormaps for the phase-coupled model (Eq. 5.57) showing (a) (total) order parameter $r$, (b) phase lag $\delta$, (c) the spontaneous drift measured by $\left|\Omega-\omega_{0}\right|$, where $\Omega$ is the wave speed (Eq. 5.55) and $\omega_{0}$ the natural frequency. (d) Area of TW state in which $\left|\Omega-\omega_{0}\right| \geq \varepsilon$, where we consider $\varepsilon=0.3$; and $\pi$-state for $\delta \geq \varepsilon$ with $\varepsilon=3$. For each point in the $70 \times 70$ grid, simulations are performed by evolving equations (5.57) considering initial conditions $\rho_{i}(0)$ and $z_{i}(0)$ randomly chosen from uniform distribution $[-1,1]$ and $\phi_{i}(0)$ uniformly distributed in the range $[-\pi, \pi]$. Other parameters: $N=10^{3}, \Delta K=1.45, \Delta G=1$, $D=0.03$, averaging over $t \in[4000,5000]$ and $d t=0.05$. Curves in the interface between incoherent and partial synchronized state are given by the condition $2 D=\langle\langle K G\rangle\rangle$. Horizontal dashed lines correspond to the critical points $K_{0 c}= \pm \Delta K / 2$. See Appendices B.4 and B.5 for additional diagrams of the phase-coupled model. . . . . . . . . . . 110 
Figure 32 - Colormaps for weighted $x$-coupled Rössler oscillators with phase-coherent $(a=0.15, b=0.2$ and $c=10)$ and funnel attractors $(a=0.2, b=0.2$ and $c=7)$ in the absence of coupling mismatches, i.e. $\Delta K=0, \Delta G=0$. Parameters: $N=10^{3}, d t=0.05$. For each $\left(G_{0}, K_{0}\right)$ in the $50 \times 50$ grid, the quantities are averaged over $\mathrm{t} \in[4700,5000]$. Initial conditions $\left(x_{i}(0), y_{i}(0), z_{i}(0)\right)$ are selected from an uniform distribution in the range $[-1,1] \ldots \ldots \ldots \ldots$. . . . . . . . . . . . . . . . . . . . .

Figure 33 - Dependence of incoherent TWs on the system size for $K_{0}=0.5, G_{0}=$ $-0.5, \Delta K=0.1, \Delta G=1$ and $D=0.03$. Each point is an average over 100 different realizations. Size of errorbars is smaller than the dots' sizes. 141

Figure 34 - Examples of time evolution of order parameters for phase-coherent oscillators in (a) $\pi$-state $\left(K_{0}=G_{0}=0\right)$, (b) lag sync $\left(K_{0}=0.25\right.$, $\left.G_{0}=-0.1\right)$; and oscillators with funnel attractor also in (c) $\pi$-state $\left(K_{0}=G_{0}=0\right)$ and $(\mathrm{d})$ lag sync $\left(K_{0}=0.03, G_{0}=0.5\right)$. Other parameters: $\Delta K=0.1, \Delta G=1, D=0.03$ and $N=10^{3}$. Shaded area corresponds to the time averaging window considered in Fig. 30 . . . . . 142

Figure 35 - Colormaps for weighted $x$-coupled Rössler oscillators with phase-coherent attractor $(a=0.15, b=0.2$ and $c=10)$. (a) $r_{1}$, (b) $r_{2}$, (c) $r$, (d) $\left|\Omega-\omega_{0}\right|$ and (e) $\delta$. Remaining parameters: $\Delta K=0.1, \Delta G=1, D=0.03$ and $N=10^{3}$. Notice in panel (d) that the white upper left and lower right regions are undefined since the phase lag is undefined. On the other hand, the frequency difference [(e)] defined via the Hilbert transform (Eq. 5.55) indicates a strict acceleration. . . . . . . . . . . . . . . 143

Figure 36 - Colormaps for phase-coupled Rössler oscillators with phase-coherent attractor $(a=0.15, b=0.2$ and $c=10)$ for $\Delta K=1.45, \Delta G=1$, $D=0.03$ and $N=10^{3}$. Concerning (d) see the remark on the phase lag in Fig 35. . . . . . . . . . . . . . . . . . 143 
Figure 37 - The absolute value of the difference between the mean phases of the two populations, $\delta$, in dependence on the mean in- and out-coupling strengths, $K_{0}$ and $G_{0}$, respectively. The two panels show zoomed regions from Fig. 31, with different transition scenarios into the TW states. In (a) the TW region is surrounded by zero-lag states, whereas in (b) the TW states are surrounded by $\pi$-states. Parameters: $N=10^{3}$, $\Delta K=1.45, \Delta G=1, D=0.03, d t=0.05$. For each $\left(G_{0}, K_{0}\right)$ in the $100 \times 100$ grid, the quantities averaged over $t \in[4000,5000]$. Dashed magenta line in panel (a) marking the interface between Incoherence and Zero-lag sync is obtained by the critical condition $2 D=\langle\langle K G\rangle\rangle$, where $\langle\langle\ldots\rangle\rangle=\int d K^{\prime} \int d G^{\prime} \ldots P\left(K^{\prime}, G^{\prime}\right)$ and $P(K, G)$ is the joint probability distribution of in- and out-couplings. Horizontal dashed lines in both panels correspond to the critical points $K_{0 c}= \pm \Delta K / 2$. . . . . . 144

Figure 38 - Colormaps for (a) $\left|\Omega-\omega_{0}\right|$ and (b) $\left|\langle\dot{\psi}\rangle_{t}-\omega_{0}\right|$ of phase-coupled Rössler oscillators. Parameters: $N=10^{3}, \Delta K=1.45, \Delta G=1, D=0.03$,

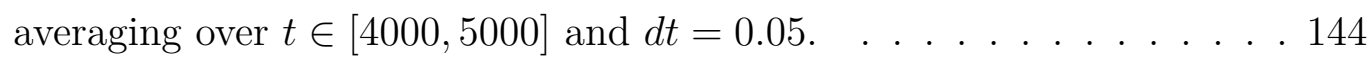

Figure 39 - Spontaneous drift measured by locking frequency $\langle\dot{\psi}\rangle_{t}$ and mean ensemble frequency $\Omega$. Parameters: $N=10^{4}, \Delta K=1.45, \Delta G=1, G_{0}=2.5$, $D=0.03$, averaging over $t \in[4000,5000]$ and $d t=0.05 \ldots \ldots 145$

Figure 40 - Order parameter $r$ and mean ensemble frequency $\Omega$ as functions of $G_{0}$ in regions of incoherence and zero-lag synchronization for oscillators with funnel attractors. Parameters: $N=10^{3}, \Delta K=0.1, \Delta G=1$, $K_{0}=0.5$ and $D=0.03 \ldots \ldots \ldots \ldots$. . . . . . . . . . . . . . . . . . . 



\section{List of Abbreviations and Acronyms}

BA Barabási-Albert

ER Erdős-Rényi

MFA Mean-field approximation

WS Watts-Strogatz 



\section{List of Symbols}

A

$\mathcal{A}$

$\alpha$

$\beta$

$c_{i}$

D

E

$k$

$\langle k\rangle$

$k_{\min }$

$k_{\max }$

$P(k)$

$\lambda$

$\lambda_{c}$

$\lambda_{c}^{\mathrm{I}}$

$\lambda_{c}^{\mathrm{D}}$

$\langle\cdot\rangle$

$\langle\cdot\rangle_{t}$

$N$

$\theta$

$\phi$

$t$

$\omega$
Adjacency matrix

Assortativity

Damping constant

Critical exponent of the phase transition

Local clustering coefficient

Noise strength

Number of edges

Degree

Network average degree

Minimum degree

Maximum degree

Degree distribution

Coupling strength

Critical coupling strength

Critical coupling strength for increasing coupling branch

Critical coupling strength for decreasing coupling branch

Ensemble average

Temporal average

Network size

Phase

Phase in the rotating frame

Time

Natural frequency 


$\begin{array}{ll}g(\omega) & \text { Natural frequency distribution } \\ \psi & \text { Mean phase } \\ \Omega & \text { Locking frequency } \\ R & \text { Guramoto order parameter } \\ r & \text { works } \\ r_{i} & \text { Cocal order parameter } \\ r_{\text {lock }} & \text { Contribution of drifting oscillators to the order parameter } \\ r_{\text {drift }} & \text { Order parameter associated to the decreasing branch } \\ r^{\mathrm{D}} & \text { Order parameter associated to the increasing branch } \\ r^{\mathrm{I}} & \text { Transitivity }\end{array}$




\section{Contents}

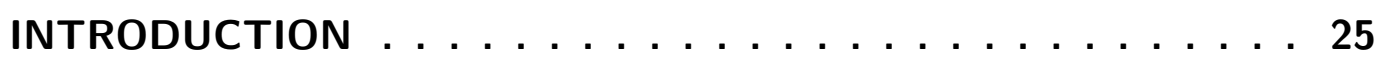

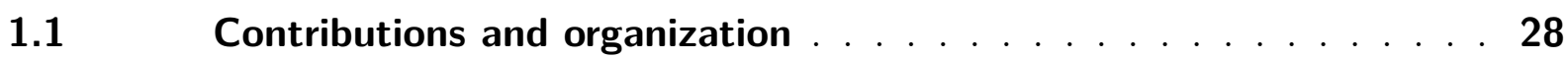

2 BASIC CONCEPTS $\ldots \ldots \ldots \ldots \ldots \ldots \ldots \ldots$

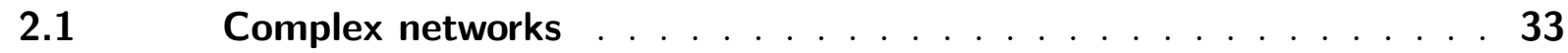

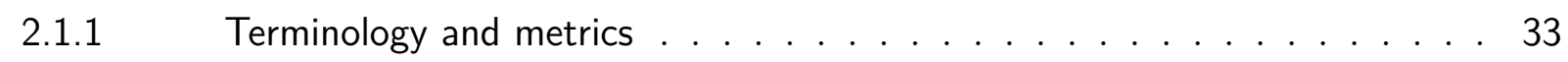

2.1.2 Network models . . . . . . . . . . . . . . . . . . . . . . . . 35

2.1.2.1 Poisson random graphs - The Erdös-Rényi model . . . . . . . . . . . . . 35

2.1.2.2 Small-world networks - The Watts-Strogatz model . . . . . . . . . . . 36

2.1.2.3 Barabási-Albert model . . . . . . . . . . . . . . . . . . 37

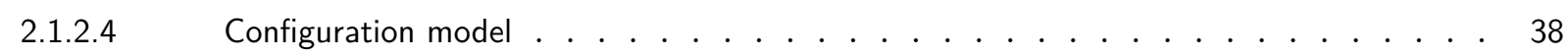

2.2 The Kuramoto model . . . . . . . . . . . . . . . . . 39

2.2.1 Mean-field theory in uncorrelated networks . . . . . . . . . . . . 42

2.2.2 The second-order Kuramoto model . . . . . . . . . . . . . . . . . 46

2.2.2.1 Mean-field theory: Fully connected graph . . . . . . . . . . . . . . . 46

3 SYNCHRONIZATION OF NETWORKS WITH NON-VANISHING TRANSITIVITY . . . . . . . . . . . . . . . 53

$3.1 \quad$ Early approaches on dynamics of clustered networks . . . . . . 54

3.2 Configuration model for clustered networks - The Newman-Miller

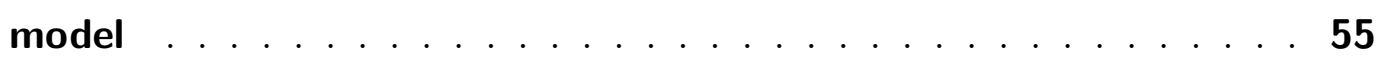

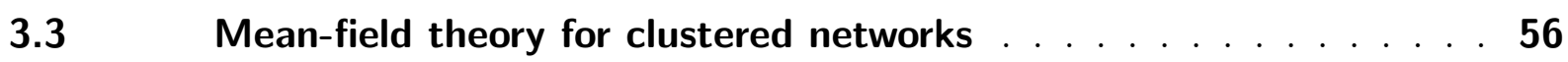

$3.4 \quad$ Numerical simulations . . . . . . . . . . . . . . . . . 58

3.5 Remarks on the effects of triangles in the Newman-Miller model . . 61

4 SECOND-ORDER KURAMOTO MODEL ON COMPLEX NET-

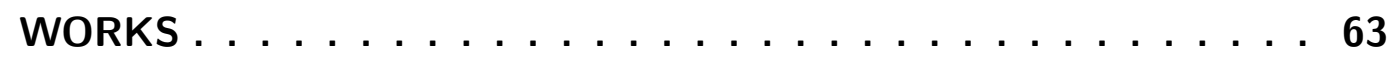

4.1 Second-order Kuramoto model on uncorrelated networks . . . . . 64

4.1 .1 The model . . . . . . . . . . . . . . . . . . . . . . 64

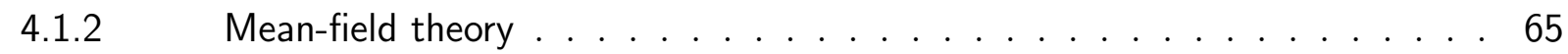

4.1 .3 Synchronization boundaries . . . . . . . . . . . . . . 67

4.1.4 Mean-field theory vs. numerical experiments . . . . . . . . . . . . . 69

4.1.5 Disturbing the correlation between frequencies and degrees . . . . . . 72

4.2 Second-order Kuramoto model in correlated networks . . . . . . 74

$4.3 \quad$ Summary and discussions $\ldots \ldots \ldots \ldots 1 \ldots \ldots$ 

INTERACTIONS . . . . . . . . . . . . . . . 83

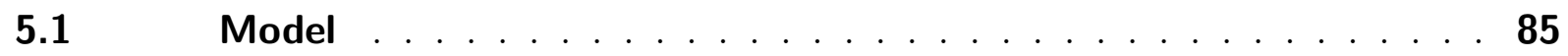

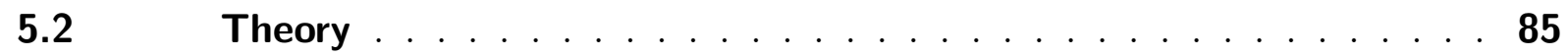

5.2.1 Gaussian approximation . . . . . . . . . . . . . . 85

5.2.1.1 Illustration - Globally coupled oscillators . . . . . . . . . . . . . . 86

5.2.1.2 Oscillators with asymmetric interactions . . . . . . . . . . . . . 88

5.2 .2 Bifurcation diagrams . . . . . . . . . . . . . . . . 92

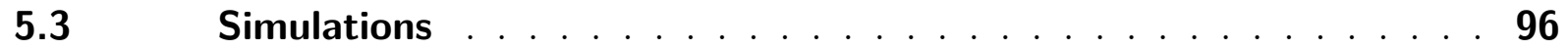

5.4 Towards complex networks with attractive and repulsive asymmetric connections . . . . . . . . . . . . . . . . . . . . 99

$5.5 \quad$ Asymmetric networks of noisy chaotic attractors . . . . . . . . 102

5.5.1 Rössler systems with diffusive coupling . . . . . . . . . . . . . . . 105

5.5.2 Phase-coupled Rössler systems . . . . . . . . . . . . . . . . . 110

5.6 Summary and outlook . . . . . . . . . . . . . . 112

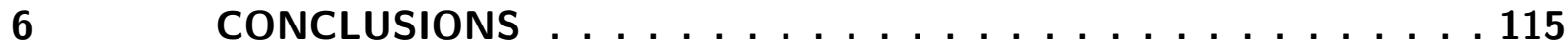

BIBLIOGRAPHY . . . . . . . . . . . . . . . . . 121

APPENDIX A - CONTRIBUTION OF DRIFTING OSCILLATORS IN THE SECOND-ORDER KURAMOTO MODEL133

APPENDIX B - ADDITIONAL DIAGRAMS FOR COUPLED RÖSSLER OSCILLATORS . . . . . . . . . . . . . 139

B.1 Incoherent traveling waves in the absence of coupling mismatch . 139

B.2 Dependence of Incoherent TWs on system size . . . . . . . . . . 139

B.3 Evolution of order parameters . . . . . . . . . . . . . . . 140

B.4 Local order parameters . . . . . . . . . . . . . . . . . . . . 140

B.5 Additional diagrams for the phase-coupled model . . . . . . . . . 141

B.6 Comparison between locking and mean ensemble frequencies . . . . 142

APPENDIX C - LIST OF PUBLICATIONS . . . . . . . . . . 147 


\section{Introduction}

Manifestations of synchronization are ubiquitously seen in natural, physical, technological and social domains.(1) The essence behind synchronization is the adjustment of rhythms of interacting oscillatory objects. Every system - regardless of its type - that exhibits periodic movement is amenable to display emergent synchronous behavior. And it is not necessary to recur to sophisticated examples to show the pervasiveness and importance of this phenomenon in our daily lives. Just remember the last time you have been to a concert. Right after the end of a piece, one notices the disordered applause, but suddenly the chaotic sound is replaced by an unison steady beat. At the same time, in the heart of each person in the audience, the coordinated frequency discharges of thousands of pacemakers cells create the rhythmic activity that sustain the cardiac cycles. These synchronous pulses find a beautiful visual analogy in the communal flashing of fireflies that have amused natural scientists for centuries $(1,2)$ - as the physician Engelbert Kaempfer vividly wrote after his excursion to Bangkok in 1680 (3): "The glowworms (...) sometimes hide their light all at once, and a moment after make it appear again with the utmost regularity and exactness (...)."

The examples above can be regarded as cases where synchronization plays a constructive role in the respective systems. There are, however, situations in which coherent motion becomes pernicious, such as in synchronous discharges patterns behind the neuronal pathologies of epilepsy $(4,5)$ and Parkinson disease.(6) The onset of collective synchronization can also be extremely dangerous in engineering, as exemplified by the incident of the London Millennium Bridge on its opening day in 2000.(2) Within minutes after being released to the public, thousands of visitors felt the bridge swaying from one side to the other. In order to not loose balance, pedestrians unconsciously tried to adjust their pace with the lateral movement, enhancing even more the deflections which reached amplitudes up to 20 centimetres.(2) Rather than being vibrations caused by, for instance, a resonance of the structure with wind (as in the famous case of the Tacoma Narrows Bridge), the phenomenon observed in London was actually a product of the positive feedback between the bridge's movement and the pace tuning of the crowd.(2) These facts not only show the fascinating similarity in the emergence of self-organized order across different realms of science, but also make it evident the vital importance in understanding how and when synchronization occurs.

The first report of synchronization is a letter dated from 26 February 1665 written by the dutch physicist Christiaan Huygens to his father.(1) In the document, Huygens writes that, while being forced to stay in his bead due to an illness, he noticed that two pendulum clocks that were hanging on the wall - he is also the inventor of such 
instruments - were swinging in harmony without any apparent reason. Intrigued with his discovery, Huygens conducted a series of experiments in order to uncover the cause of this "odd kind of sympathy".(1) The set up involved hanging two clocks with different physical dimensions on a common wooden beam and with controllable distances from one another. As described in his letters, when placed sufficiently far apart, noticeable differences in the clocks' pace were measured. Yet, insofar as they were brought closer, synchrony was restored and persisted for days. Interestingly, even if intentionally perturbed, the sympathetic movement would be reestablished within a short time. At first, Huygens' guess was that air currents shared by the pendula were causing the synchronization. However, after performing tests with boxes to prevent any passage of air, he realized that the agreement between the clocks was due to imperceptible movements of the beam; or, in modern terminology, mutual synchronization arose by virtue of coupling. With almost accidental observations, it was then inaugurated the field of coupled oscillators. Remarkably, despite the long time of these findings, Huygens' experiments are still a matter of scrutiny in modern times. For recent experimental advances on synchronization of clocks we refer the reader to $(7,8)$. Other notorious historical moments in the discovery of synchronization include the observation of frequency locking of pairs of organ-pipes by Lord Rayleigh, and the detection of synchronization properties of triode generators by Eccles and Vincent.(1)

Although the first report on a synchronization phenomenon dates back to Huygens in the 17th century (1), the topic of spontaneous emergence of collective behavior in large populations of oscillators was only brought to higher attention after the work by Wiener $(9,10)$. Wiener was interested in explaining the generation of frequencies closer to $10 \mathrm{~Hz}$ - the so called alpha rhythms - in electroencephalogram (EEG) exams. His intuition was that this particular phenomenon was somehow related with the same mechanism behind the coherent behavior in other biological systems, such as in the synchronous flash of fireflies. More precisely, he hypothesized that these alpha rhythms were caused by interactions of populations of oscillators in the brain. The idea was that the natural frequencies of these oscillators were distributed around $10 \mathrm{~Hz}$ and that this common rhythm would be achieved through a self-organized process of frequency adjustment. That is, in this hypothetical scenario, oscillators running too fast would have theirs frequencies reduced to the average, and slower ones would be forced to speed it up. To support these claims, Wiener used experimental data available at his time and further developed a mathematical approach that turned out to be unfruitful. $(2,11)$

Wiener's idea was interesting and anticipating, but at the same time too complex to get analytical insights from it. A more promising approach was later developed by Winfree $(12,13)$, who was the first to properly state the problem of collective synchronization mathematically. Winfree realized that the modelling of biological rhythms should be treated differently from traditional problems in mechanics that have been studied for 
many years in physics. He understood that biological oscillators should be self-sustained and non-identical (instead of being conservative and identical); and interactions should be considered to be weak in the sense that the influence of coupling should not drive oscillators away from their limit-cycle. In other words, the external stimuli to a given oscillator is required to not alter its amplitude, but only its period. Under these assumptions, Winfree formulated a model in which oscillators are described solely in terms of their phases and have distributed natural frequencies as a way to incorporate biological variability. $(12,13)$ By performing numerical simulations, Winfree found that spontaneous synchronization emerges as a threshold process akin to a phase transition. His main finding was: If the spread of the frequencies is higher compared to the coupling between the oscillators, then each oscillator would run at its own natural frequency, causing the population to behave incoherently. On the other hand, in case the coupling is sufficiently strong to overcome the heterogeneity in the frequencies, then the system spontaneously locks into synchrony.(12)

Deeply motivated by these results and the interesting analogy with statistical physics, in 1975 Kuramoto simplified Winfree's approach to obtain a solvable model preserving the fundamental assumption of having oscillators with distributed frequencies. $(14,15)$ The key approximation in what latter would be known as the Kuramoto model was the replacement of the generic coupling function in Winfree's model by a sinusoidal coupling depending on the phase difference between two interacting oscillators. Besides providing great analytical tractability, the Kuramoto model established itself as an unified framework to quantitatively and qualitatively analyse synchronization phenomena in a wide variety of complex systems.(16)

The results obtained by Kuramoto $(14,15)$ made a huge impact on the scientific community and gave rise to a plethora of extensions and fundamental new discoveries.(11, 16) However, although the paradigm introduced in $(14,15)$ was successful in explaining the onset of synchronous behavior in large populations, the model was still relying on a crucial unrealistic assumption: The global coupling. In the same way that identical oscillators seems as a rather strong condition to describe biological systems, all-to-all connectivity also appears to be hardly observed in nature. However, a twist in coupled oscillators theory happened in 1998. In that year, Watts and Strogatz (17) introduced a simple yet ingenious coupling scheme initially intended to study the synchronous chirping of crickets. Precisely, their original goal was to uncover how the inclusion of shortcuts would affect the ability to synchronize of crickets placed in a regular lattice. Such a experiment was never performed by the authors, but their study on the topological properties of the proposed network model became a milestone in the theory of complex systems as being one of the starting points of modern network science.(18)

For decades the research on coupled oscillators has been concentrated on the complexity of the nonlinear dynamics of the models without the concern of including further 
complexity arising from heterogeneity in the connections among oscillators. $(11,16)$ On the other hand, since the work by Watts and Strogatz (17), an overwhelming number of evidences ${ }^{1}$ has been published showing that the architecture of real-world networks is far from being as simple as the fully connected and regular topologies for long considered in nonlinear sciences. The rise of the so-called complex networks has then introduced a new dimension in the study of dynamical systems. Researchers started to be puzzled by the question of how the connectivity pattern between the elements in a network can influence the performance of a given dynamical process. It is in this context that the present thesis is inserted. In particular, we will address fundamental issues regarding the interplay between structure and collective dynamics of Kuramoto oscillators coupled in complex networks. In the next section, we state the main research questions addressed here.

\subsection{Contributions and organization}

The present work is dedicated to the investigation of fundamental problems regarding the collective dynamics of Kuramoto oscillators in complex networks. In particular, this thesis has three main broad themes which are comprised in Chapters 3, 4 and 5. We explain our goals and contributions in detail below and subsequently, in Chapter 2, we provide an overview on the important terminology and basic concepts that will be used throughout this thesis.

The highest goal in network science has been the accurate modelling of real complex systems. This task includes the characterization of their structure as well as the comprehension of dynamical processes on top of them. Unfortunately, progresses in the latter topic have been much more slower than in the former. More precisely, while the topology of real-world networks has been extensively mapped and analysed through numerous sets of methods and metrics $(18,21)$, it is still not clear how precisely certain types of structural patterns affect network dynamics. The reason for this resides in the fact that dynamical processes are usually studied by considering networks generated through random models. Although this methodology allows great analytical tractability, network models mimic the architecture of real-world networks only up to a certain extend. That is, while certain salient topological characteristics are indeed reproduced, many other distinguishing features are neglected. Of particular interest is the property of clustering (18), which refers to the tendency of groups of three nodes to form a fully connected subgraph or, in other words, a triangle. As we shall see, triangles can be seen as the simplest structural pattern that is omnipresent in real-world networks, but absent in the majority of random network models. (18) Thus, if the thorough understanding of real network is sought, it is

$\overline{1}$ For a comprehensive list of references, see, for instance, the classical reviews (19-23) and books (18, $24,25)$. 
mandatory to take into account realistic network features such as clustering in the models employed in the study of dynamical processes. We will tackle this issue in the context of synchronization of phase oscillators. Precisely, in Chapter 3 we will address the following question

- How do triangles affect the emergence of synchronization?

To this end, we will consider a recent generalization of the traditional configuration model that is able to create networks with tunable levels of clustering while maintaining other network metrics constant. Furthermore, in order to analytically assess the synchronous behavior of clustered and unclustered networks, we will generalize to the latter model the traditional heterogeneous degree mean-field description of coupled phase oscillators. Chapter 3 also provides an overview of the recent alternative approaches to account for clustering in network dynamics against which we will contrast our original results.

Following the same canon of Chapter 3, Chapter 4 is also devoted to a subject relevant for the study of real-world networks. One of the most paradigmatic examples of man-made systems that crucially rely on the synchronous operation of its units are power grids. Under normal conditions, power generators and consumers operate in a stable synchronous state; however, such a reliable functioning is constantly subjected to threats such as structural or human failures, intentional attacks or natural disasters which can trigger massive blackouts with potentially terrible consequences and economic losses.(2629) Usually, blackouts happen as cascade processes. At first, unexpected events cause single elements in the power grid, such as transmission lines and generators, to collapse. This failure can then induce the desynchronization of neighbouring units, leading them to no longer operate at the grid's rated frequency $(50$ or $60 \mathrm{~Hz}$ ). These voltage oscillations may in turn force components to be switched off, provoking a chain reaction in which more nodes in the grid are destabilized and finally culminating in a large blackout.(26-29) How fast these events propagate through the system and the extension of the damage produced are points intrinsically related with the connectivity pattern of the grid. In other words, the ability of a power grid to withstand failures crucially depends on its underlying network topology. Therefore, it is of ultimate importance to deepen the understanding of how structure and dynamics are related in this context.

A suitable way to evaluate the large scale dynamics of power grids is by virtue of a variant of the Kuramoto model that includes inertial effects in the phases' evolution - the second-order Kuramoto model.(30,31) Surprisingly, coupled second-order oscillators have attracted considerable less attention in the network synchronization field than its classical version.(32) Given this gap in the literature, we will take a step back in the analysis of real power grids and rather focus on theoretical aspects of the model in general networks. In particular, Chapter 4 is devoted to the question: 
- How does the presence of inertia change the collective dynamics of Kuramoto oscillators in a complex network?

To answer it, we firstly extend the self-consistent analysis carried out by Tanaka et al. $(30,31)$, who are the first authors to adapt Kuramoto's original calculations to the problem of inertial oscillators in the fully connected graph. More precisely, we generalize the calculations in $(30,31)$ so as to take into account the influence of network topology. This contribution in Chapter 4 consists in the first mean-field treatment of second-order Kuramoto oscillators in uncorrelated networks.(33) Besides these theoretical developments, we also investigate the collective dynamics of inertial oscillators in the presence of degree-degree correlation by performing extensive numerical simulations. By doing so, we uncover an interesting dynamical equivalence between changes in network structure associated with degree mixing properties and variations in the oscillators' damping coefficient.

In Chapter 5 we give attention to another particularity of the network structure - we study the impact of the nature of the connections on the overall network dynamics. Chapters 3 and 4 are restricted to analyze populations of oscillators connected via positive and symmetric couplings in the sense that nodes always feel a positive response coming from the rest of the population. Indeed, this mutual attraction of phases is the essence behind the spontaneous transition from incoherence to partially synchronous states; that is, as the coupling becomes stronger, more oscillators lock in coherent motion, increasing the influence of the mean-field on the oscillators. This process creates a positive feedback between coherence and coupling which then leads to the formation of a macroscopic synchronous component. $(11,15)$ However, what could be expected if the mean-field is set to repel oscillators in a nonuniform fashion? In Chapter 5, we consider the general case in which nodes interact through asymmetric (directed) attractive and negative couplings. More precisely, in the last part of our investigations, we address the question

- What other collective dynamical states can emerge if network connections cease to be purely symmetric and attractive?

Or, in other words, is it possible to observe other states different from the classical incoherent and partially synchronized states? As will become clear later, the motivation for such an endeavour is rooted in the seminal work on "oscillator glasses" by Daido (34), and analogies of phase oscillator models with excitatory and inhibitory interactions in neuronal networks (35), and models of opinion formation.(36) Also in this chapter, we enhance the level of complexity in our model by including the influence of stochastic forces in order to take into account realistic aspects present in experimental situations. (37) This characteristic further allows us to employ the so-called Gaussian approximation (38) technique through which a detailed bifurcation analysis is achieved. As we shall see, peculiar 
patterns of synchronization are uncovered as a consequence of the coexistence of positive and negative responses to the populations' mean-field.

Finally, Chapter 6 presents our overall conclusions. 



\section{Basic concepts}

\subsection{Complex networks}

In this section we shall introduce the relevant concepts of network science that will be used throughout this thesis. The list of network metrics and generating models below is by far not exhaustive. For a comprehensive review on this topic we refer the reader to classical texts in the field (see, for instance, $(18,20,21,39)$ ).

\subsubsection{Terminology and metrics}

Networks are mathematically modelled as a set of nodes $\mathcal{V}$ and a set edges $\mathcal{E}$ connecting the nodes. Vertex and link are terms that are often used as synonyms for nodes and edges, respectively. In this thesis we consider that each node is labelled as $i \in\{1, \ldots, N\}$, where $N$ is the total number of nodes. We will also refer to $N$ as the size of the network. A convenient way to represent a network is in terms of the adjacency matrix A, whose elements are defined as

$$
A_{i j}= \begin{cases}1 & \text { if nodes } i \text { and } j \text { are connected by an edge } \\ 0 & \text { otherwise }\end{cases}
$$

When the adjacency matrix is symmetric $\left(A_{i j}=A_{j i}\right)$ the network is called undirected.

The number of connections attached to a given node is called its degree and it is expressed as

$$
k_{i}=\sum_{j=1}^{N} A_{i j}
$$

The network average degree is then given by

$$
\langle k\rangle=\frac{1}{N} \sum_{j=1}^{N} k_{i} .
$$

An important quantity related to the statistical characterization of networks is the degree distribution $P(k)$, which measures the probability that a randomly selected node has degree $k$. Furthermore, the $n$-th moment of the degree distribution is defined as

$$
\left\langle k^{n}\right\rangle=\sum_{k} k^{n} P(k) .
$$

A network feature that will be recurrent in these pages is the property of clustering, which denotes the propensity that neighbours of a given node are also neighbours of 
themselves.(18) A straightforward way to quantify clustering is by calculating the local clustering coefficient that is the probability that two nodes $j$ and $k$ are connected given that both are connected to $i$ forming a triangle (17):

$$
\begin{aligned}
c_{i} & =\frac{\text { number of triangles attached to node } i}{\text { number of triples intermediated by node } i} \\
& =\frac{2 \sum_{k>j} A_{i j} A_{i k} A_{j k}}{k_{i}\left(k_{i}-1\right)}
\end{aligned}
$$

where a triple is a sequence of three nodes $\{i, j, k\}$ connected by two edges. Triangles can also be referred as cycles of order three.

On the global scale, the clustering of the network can be measured by the respective average of $c_{i}$, i.e. $\left\langle c_{i}\right\rangle=N^{-1} \sum_{j=1} c_{i}$; or by the network transitivity ${ }^{1}$

$$
\mathcal{T}=\frac{3 \times(\text { number of triangles in the network })}{\text { (number of connected triples })}=\frac{3 N_{\triangle}}{N_{3}}
$$

The vast majority of real-world networks tends to exhibit significantly higher levels of clustering than those observed in random network models.(41) This fact will be particular relevant to motivate our study in Chapter 3 on the impact of the occurrence of triangles on the collective dynamics of phase oscillators. Noteworthy, the expression "clustered network" will be employed throughout this thesis to refer to networks that posses nonvanishing values of $\mathcal{T}$ or $\left\langle c_{i}\right\rangle$.

Networks can also be characterized in term of degree-degree correlations (42), which in turn can be analyzed by means of the conditional probability $P\left(k^{\prime} \mid k\right)$, that is the probability that a node of degree $k$ is connected with a node of degree $k^{\prime}$. In uncorrelated networks, $P\left(k^{\prime} \mid k\right)$ does not depend on $k$ and the only relevant function for networks characterization is $P(k)$.(42) Due to the difficulties in estimating the conditional probability of degree-degree connectivity in empirical data (18), researchers commonly use the assortativity coefficient $\mathcal{A}$ as a measure of degree-degree correlation. The correlation between a pair of random variables is calculated by taking into account the Pearson correlation coefficient. It is then natural to recur to this approach to determine the correlations in networks (43), i.e.:

$$
\mathcal{A}=\frac{\frac{1}{E} \sum_{j>i} k_{i} k_{j} A_{i j}-\left[\frac{1}{E} \sum_{j>i} \frac{1}{2}\left(k_{i}+k_{j}\right) A_{i j}\right]^{2}}{\frac{1}{E} \sum_{j>i} \frac{1}{2}\left(k_{i}^{2}+k_{j}^{2}\right) A_{i j}-\left[\frac{1}{E} \sum_{j>i} \frac{1}{2}\left(k_{i}+k_{j}\right) A_{i j}\right]^{2}},
$$

1 One could be tempted to state that $\left\langle c_{i}\right\rangle$ and $\mathcal{T}$ can be employed interchangeably to quantify how globally clustered a network is, since both measures are bounded between 0 and 1 , and are, apparently quantifying the same property. However, we should notice that high average local clustering does not necessarily imply high values of transitivity. In fact, there is a series of real-world networks that exhibit appreciate values of $\left\langle c_{i}\right\rangle$ with $\mathcal{T}$ being significantly lower. Curiously, it is also possible to show that, for a particular class of network known as windmill graphs, $\left\langle c_{i}\right\rangle \rightarrow 1$ whereas $\mathcal{T} \rightarrow 0$, in the limit $N \rightarrow \infty(40)$. 
where $E$ is the total number of edges in the network.

Another fundamental concept in networks is the length of a geodesic path $d_{i j}$, which is the minimum number of edges that a walker must cross to reach node $j$ departing from $i$. The average of all geodesic path lengths is referred as the average shortest path length and it is given by (18)

$$
\ell=\frac{1}{N(N-1)} \sum_{i \neq j} d_{i j} .
$$

Many important topological and dynamical properties of networks are directly related with the quantity above. For instance, a network is said to be small-world as long as the average shortest path length shows a scaling $\ell \sim \log N$ as $N \rightarrow \infty$.(18) We comment more about this in the next section.

\subsubsection{Network models}

In network theory, a model is a set of rules for the generation of an ensemble of graphs, usually with the goal to reproduce some aspects encountered in some real systems. This section reviews the most studied random network models in the context of dynamical process and which will be constantly mentioned in this work.

\subsubsection{Poisson random graphs - The Erdős-Rényi model}

In a series of papers (44-47) between the 1950s and 1960s, the Hungarian mathematicians Paul Erdős and Alfréd Rényi (ER) established the foundations of modern network science by introducing probabilistic methods in classical graph theory. The networks studied by them are generated by the following mechanism: Starting with $N$ disconnected nodes, edges are placed between pairs of nodes with probability $p$. The set of all possible networks created by repeating this process is called the $G_{N, p}$ ensemble. We will refer to members of this ensemble as ER networks.

Since each node is connected to the rest $N-1$ other vertices with probability $p$, the average degree is then given by $\langle k\rangle=p(N-1)$. Furthermore, one can show that in the thermodynamic limit $N \rightarrow \infty$ the degree distribution converges to (18)

$$
P(k)=\frac{\langle k\rangle^{k} e^{-\langle k\rangle}}{k !},
$$

and that the average shortest path length scales as (18)

$$
\ell \sim \frac{\log N}{\log \langle k\rangle}
$$

The process of establishing a connection in networks created by the ER model is completely random, in striking contrast with real-world structures, where usually internal 
rules of the underlying real system is the main factor driving the link assignment. $\mathrm{Cu}$ riously, despite this intrinsic difference, the estimative of $\ell$ indeed fits with the values observed in some social networks. (18) However, this is the only similarity that these systems share. For instance, in the limit $N \rightarrow \infty$, it is possible to demonstrate that $\mathcal{T} \rightarrow 0$, which by no means reflects the very clustered topology of a wide variety of networks found in nature.(18)

Although we started this section mentioning that the conception of a random graph model is usually made having in mind a given aspect present in real systems, Erdös and Rényi had in fact no intentions in creating a realistic network representation. They were purely interested in the mathematical puzzle of unveiling the topological properties of a discrete system in which connections between its elements arise by chance. Nevertheless, it is worth mentioning an interesting preceding paper by the Russian mathematicians Anatol Rapoport and Ray Solomonoff.(48) There, using neuronal networks as a metaphor, the authors considered a particular kind of bond percolation problem in which axons (links) are randomly assigned to pairs of neurons (nodes). The network model proposed in (48) turns out to be similar to the one proposed later by Erdős and Rényi; actually Rapoport and Solomonoff even predicted the critical condition $\langle k\rangle_{c}=1$ for the emergence of a giant connected component later recalculated much more rigorously by the Hungarians researchers. But perhaps even more remarkable is the outlook given in (48) - the authors speculated that their findings could be potentially useful in problems concerning the connectivity of neuronal networks, mathematical genetics and spread of diseases in social systems. It was then anticipated, in a surprising way, questions extensively investigated in nowadays network science. Noteworthy, even though there are a few contributions (49-51) addressing random graphs prior to Erdős and Rényi, the credit for the foundation of random graph theory is still attributed to the latter authors. As remarked by Bollobás (52), it was only in (44) that the formalism employed today was introduced, whereas in previous papers the approaches were rather deterministic and focused on enumeration problems.

\subsubsection{Small-world networks - The Watts-Strogatz model}

In the point of view of designing models for real-world topologies, the ER networks have the drawback of lacking of clustered structure. Although the scale of the geodesic distances reproduced by the model is reasonable with some empiric observations, the transitivity levels are too unrealistic. In 1998, this problem left by the ER model seemed to be solved by Watts and Strogatz (WS), who introduced (17) a new class of random graph model known as the small-world network (or WS model). Curiously, as mentioned in the introduction part, its discovery is intimately related with the study of synchronization of oscillators. Inspired by the experiments of Stanley Milgram in the 1960s (53) and stories of the "six degrees of separation" in pop culture (2), the authors in (17) started to wonder 
about the effect of reducing the average shortest length on the collective dynamics of biological oscillators. Their goal was to develop a network structure that could be tuned between completely random and fully ordered via controlling a single parameter. The simple algorithm to construct such a WS network is given by the following steps:

1. Place the nodes in a one-dimensional ring with periodic boundary conditions.

2. Connect every node to its respective $k$ nearest neighbours in both directions so that $k_{i}=2 k \forall i$.

3. Rewire each edge with probability $p$.

For $p=0$ we have the initial perfectly ordered ring, while $p=1$ generates fully randomized networks similar to the ER model, but with slightly different degree distribution. The distinguishing feature of WS model lies in intermediate values of $p$. Precisely, for $p \in[0.01,0.05]$ the networks constructed are said to belong to the small-world regime, which is characterized by the coexistence of high values of $\mathcal{T}$ with distances of order $\ell \sim \log N$. In (17), the authors further compared the estimates obtained through their model with calculations of $\mathcal{T}$ and $\ell$ for the electrical power grid of the western United States, the collaboration graph of film actors and the neuronal network of the nematode worm C. elegans. Interestingly, the measures obtained with the WS networks were compatible with the ones empirically observed. Given that the real networks considered in (17) belong to different domains (social, technological and biological), the small-world paradigm appeared to be a potential candidate for an unifying feature of many complex systems. However, the model by Watts and Strogatz suffers from the shortcoming of yielding too narrow degree distributions. This consisted in a huge discrepancy in describing real degree distributions specially after the discovery of the highly heterogeneous topology of scale-free networks.

\subsubsection{Barabási-Albert model}

Almost concomitantly with the publication of (17), many empirical evidences started to appear suggesting that the distribution of connections in real networks can actually greatly deviate from the single peaked distributions reproduced by ER and WS models.(54-56) These studies showed that systems such as the World Wide Web and networks of scientific citation have highly-skewed degree distributions that are more accurately fitted by power laws as

$$
P(k) \sim k^{-\gamma}
$$

Networks whose degree distribution follow Eq. 2.11 are usually termed scale-free networks, denoting the scale invariance of the argument of the power law function. This scale-free 
character has profound implications in the topological organization of nodes. For instance, in ER networks, the distribution of degrees is highly concentrate around the average $\langle k\rangle$; that is, for Poisson choices of $P(k)$, the standard deviation is given by $\sigma_{k}=\sqrt{k}$, thus almost the entire network has degrees in the range $k=\langle k\rangle \pm \sqrt{\langle k\rangle}$. In other words, $\langle k\rangle$ is a well defined scale in the system. The same is valid for the WS model. Scale-free networks with $\gamma<3$, on the other hand, have a divergent second moment $\left\langle k^{2}\right\rangle$ as $N \rightarrow \infty$, meaning that fluctuations around the average can be arbitrary large. Thus, when selecting a node uniformly at random, any outcome for its degree can be expected. In this sense, the average degree tells us no useful information and the network is said to be "scale-free".

The first network model designed with the aim to reproduce the scale-free property of real networks is by Barabási and Albert (BA) (57), and it relies on two basic assumptions: First, the network is no longer treated as static, instead it is considered to be a living organism, with new nodes constantly being added at each step of the generating process; and second, new nodes tend to link preferentially to well connected nodes. The reproduction of the scale-free nature of real-world networks by the growth and preferential attachment mechanism represents, together with the WS model, a great breakthrough in the theory of networks. However, although very insightful, the BA model is restricted in the creation of networks with a single power law exponent, namely $\gamma=3$. While generalizations of the classical preferential attachment have been proposed in order to account for different values of $\gamma$, none of these variations escape from the curse of lacking clustering in the thermodynamic limit.(18)

\subsubsection{Configuration model}

The three previously described models have one characteristic in common: No matter how we change the parameters in them, the resulted degree distribution is always restricted to a single class of function - Poisson, scale-free or binomial in the WS model. This can be rather unfortunate if one seeks more flexibility in modelling the heterogeneity of connections of some observed network. A solution to this problem is provided by Molloy and Reed with the so-called configuration model.(58) In this model, instead of setting the linking mechanism in order to obtain the network topology a posteriori, we first choose a desired degree distribution $\tilde{P}(k)$ from which a degree sequence $\left\{k_{1}, k_{2}, \cdots, k_{N}\right\}$ is drawn. In other words, we first choose the level of heterogeneity that we want our network to have. To construct the network, we must then join the "half-edges" (or stubs, as commonly referred in network literature (18)) uniformly at random in order to match with the potential degrees in the original degree sequence. At the end of this process, the obtained degree distribution $P(k)$ will not be exactly as the one prescribed $\tilde{P}(k)$; however the larger the networks, the better the agreement between them.

Networks constructed via the above described mechanism can be useful as null- 
models to investigate whether a certain topological or dynamical property of a network is a product arising by pure chance or if it is due to a phenomenon related to its functioning. Examples of the use of such a methodology can be found, for instance, in the detection of motifs (59), which are subgraph patterns that are detected in real networks but are unlike to be formed in synthetic ones. Unfortunately, the degree distribution is the only property of a real network that the model is able to mimic; clustering and degree-degree correlations tend to vanish as the size $N$ diverges. We should not, however, downsize the value of this model in face of this fact. Thanks to its great analytical tractability, one has witnessed a tremendous progress in the study of several dynamical processes on heterogeneous topologies in the last years.(39) Furthermore, advances have recently started to be achieved towards extensions of the model (60) designed to incorporate more elaborated topological features such as high clustering. Section 3.2 will introduce the pioneer of this class of models and later in Sec. 3.3 we will use it as a substrate to study the dynamics of phase oscillators the presence of non-null values of clustering.

\subsection{The Kuramoto model}

The Kuramoto model consists of a population of $N$ phase oscillators whose evolution is dictated by the governing equations $(14,15)$

$$
\dot{\theta}_{i}=\omega_{i}+\frac{\lambda}{N} \sum_{j=1}^{N} \sin \left(\theta_{j}-\theta_{i}\right), \quad i=1 \ldots, N,
$$

where $\theta_{i}$ denotes the phase of the $i$ th oscillator, $\lambda$ is the coupling strength and $\omega_{i}$ the natural frequencies, which are distributed according to a given probability density $g(\omega)$. In his original approach, Kuramoto considered $g(\omega)$ to be unimodal and symmetric centered at $\omega=\bar{\omega}$, which can be assumed to be $\bar{\omega}=0$ after a shift. In the examples of this chapter, we also consider $\bar{\omega}=0$ and symmetric choices of $g(\omega)$, without loss of generality. Kuramoto further introduced the order parameter $(14,15)$

$$
R e^{i \psi(t)}=\frac{1}{N} \sum_{j=1}^{N} e^{i \theta_{j}(t)},
$$

in order to quantify the overall synchrony of the population. This quantity has the interesting interpretation of being the centroid of a set of $N$ points $e^{i \theta_{j}}$ distributed in the unit circle in the complex plane. If the phases are uniformly spread in the range $[0,2 \pi]$ then $R \approx 0$ meaning that there is no synchrony among the oscillators. On the other hand, when all the oscillators rotate grouped into a synchronous cluster with the same average phase $\psi(t)$ we have $R \approx 1$. We can rewrite the set of Eqs. 2.12 using the mean-field quantities $R$ and $\psi$ by multiplying both sides of Eq. 2.13 by $e^{-i \theta_{i}}$ and equating the imaginary parts 
to obtain

$$
\dot{\theta}_{i}=\omega_{i}+\lambda R \sin \left(\psi-\theta_{i}\right)
$$

In this formulation, the phases $\theta_{i}$ seem to evolve independently from each other, but the interaction is actually set through $R$ and $\psi$. Furthermore, note that the effective coupling is now proportional to the order parameter $R$, creating a feedback relation between coupling and synchronization. More specifically, small increments in the order parameter $R$ end up by increasing the effective coupling in Eq. 2.14 attracting, in this way, more oscillators to the synchronous group. From this process, a self-consistent relation between the phases $\theta_{i}$ and the mean-field is found, i.e. $R$ and $\psi$ will define the evolution of $\theta_{i}$, but at the same time, the phases $\theta_{i}$ self-consistently yield the mean-field through Eq. 2.13.

In the limit of infinite number of oscillators, the system can be described by the probability density $\rho(\theta, \omega, t)$ so that $\rho(\theta, \omega, t) d \theta$ gives the fraction of oscillators with phase betweeen $\theta$ and $\theta+d \theta$ at time $t$ for a given natural frequency $\omega$. Since $\rho$ is nonnegative and $2 \pi$-periodic in $\theta$, we have that it satisfies the normalization condition

$$
\int_{-\pi}^{\pi} \rho(\theta, \omega, t) d \theta=1
$$

Furthermore, the density $\rho$ should obey the continuity equation

$$
\frac{\partial \rho}{\partial t}+\frac{\partial}{\partial \theta}(\rho v)=0
$$

where $v(\theta, \omega, t)=\omega+\lambda R \sin (\psi-\theta)$ is the angular velocity of a given oscillator with phase $\theta$ and natural frequency $\omega$ at time $t$. In the continuum limit, the order parameter $R$ and the average phase $\psi$ defined in Eq. 2.13 are determined in terms of the probability density $\rho(\theta, \omega, t)$ as

$$
R e^{i \psi(t)}=\int_{-\pi}^{\pi} \int_{-\infty}^{\infty} e^{i \theta} \rho(\theta, \omega, t) g(\omega) d \omega d \theta
$$

Equations 2.16 and 2.17 admit the trivial solution $R=0$, which corresponds to the stationary distribution $\rho=1 / 2 \pi$, characterizing the incoherent state. In the partial synchronized state $(0<R<1)$, the continuity equation (2.16) yields in the stationary regime $(\partial \rho / \partial t=0)$

$$
\rho(\theta, \omega)= \begin{cases}\delta\left[\theta-\psi-\arcsin \left(\frac{\omega}{\lambda R}\right)\right], & \text { if }|\omega| \leq \lambda R \\ \frac{\sqrt{\omega^{2}-(\lambda R)^{2}}}{2 \pi|\omega-\lambda R \sin (\theta-\psi)|} & \text { otherwise. }\end{cases}
$$

The solutions for the stationary distribution assert that in the partial synchronized state the oscillators are divided into two groups. Specifically, those with frequencies $|\omega| \leq \lambda R$ correspond to the oscillators entrained by mean-field, i.e. the oscillators that evolve locked in a common average phase $\psi(t)=\Omega t$, where $\Omega$ is the average frequency of the population. On the other hand, the oscillators with $|\omega|>\lambda R$ (referred as drifting oscillators) rotate incoherently. Inserting the stationary distributions in Eq. 2.17, one obtains the 


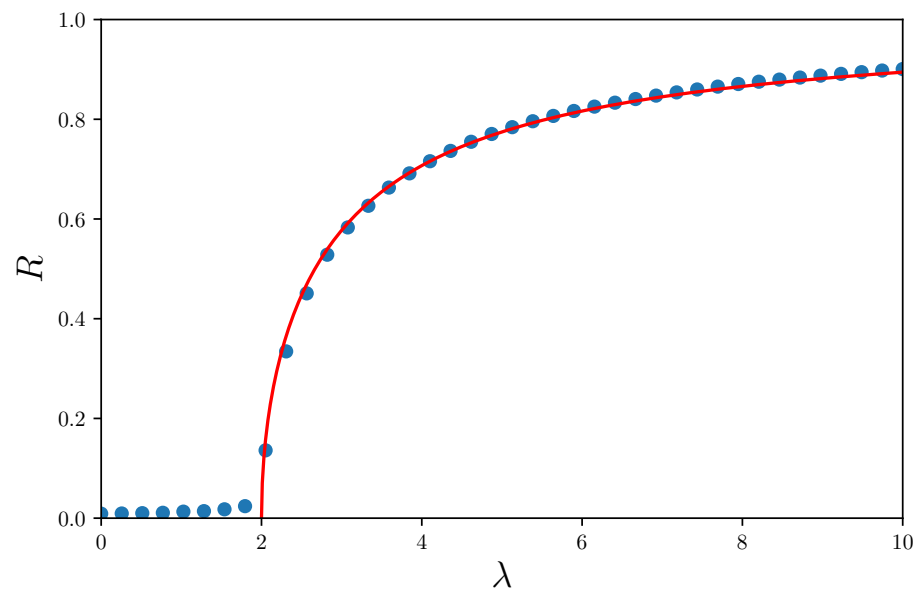

Figure 1 - Typical dependence of the order parameter $R$ as function of the coupling $\lambda$ for a population of first-order Kuramoto oscillators coupled in the fully connected graph considering $g(\omega)=\left[\pi\left(\omega^{2}+1\right)\right]^{-1}$. Dots are obtained by numerically evolving Eqs. 2.12 with $N=10^{4}$ oscillators. Solid line is derived by solving Eq. 2.19.

Source: By the author.

self-consistent equation for $R$

$$
R=\lambda R \int_{-\pi / 2}^{\pi / 2} \cos ^{2} \theta g(\lambda R \sin \theta) d \theta
$$

where the integral that corresponds to the contribution of drifting oscillators vanished due to $g(\omega)=g(-\omega)$ and the symmetry $\rho(\theta+\pi,-\omega)=\rho(\theta, \omega)$. By letting $R \rightarrow 0^{+}$in Eq. 2.19, we get

$$
\lambda_{c}^{\mathrm{KM}}=\frac{2}{\pi g(0)},
$$

which is the critical coupling strength for the onset of synchronization firstly obtained by Kuramoto.(14,15) Moreover, expanding the right-hand side of Eq. 2.19 in powers of $\lambda R$, given that $g^{\prime \prime}(0)<0$, yields

$$
R \sim \sqrt{\frac{-16\left(\lambda-\lambda_{c}\right)}{\pi \lambda_{c}^{4} g^{\prime \prime}(0)}},
$$

for $\lambda \rightarrow \lambda_{c}$. Thus, near the transition point, the order parameter yields the form $R \sim$ $\left(\lambda-\lambda_{c}\right)^{\beta}$ with $\beta=1 / 2$, clearly showing an analogy to a second-order phase transition observed in magnetic systems. Figure 1 shows a typical behavior of the order parameter $R$ as coupling $\lambda$ is varied considering Lorentzian natural frequency distribution $g(\omega)=$ $\left[\pi\left(\omega^{2}+1\right)\right]^{-1}$.

Besides being a timely contribution for the understanding of how synchronization in large populations of mutually coupled oscillators sets in, Kuramoto's analysis also established a link between mean-field techniques in statistical physics and nonlinear dynamics. However, although undoubtedly brilliant and insightful, his approach was not rigorous 
and left many questions that puzzled researchers throughout the following years $(16,61)$, making it still a matter of fundamental research in recent times.(62-68)

In parallel with the studies on the traditional Kuramoto model, one has witnessed the emergence of an overwhelming number of works focused on particular effects caused by the introduction of heterogeneous connection patterns, letting the interactions to be no longer only restricted to global coupling. In the next section we discuss the required approximations to analytically treat the problem in uncorrelated networks, i.e. networks in which the degree of connected nodes are not correlated ( $\mathcal{A}=0$ in Eq. 2.7). Subsequently, we will treat the second-order Kuramoto on the fully connected graph.

\subsubsection{Mean-field theory in uncorrelated networks}

The generalization of the Kuramoto model in complex networks is obtained by including the connectivity in the coupling term as

$$
\dot{\theta}_{i}=\omega_{i}+\sum_{j=1}^{N} \lambda_{i j} A_{i j} \sin \left(\theta_{j}-\theta_{i}\right)
$$

The mere definition of the model (2.22) carries together the first problem when treating phase coupled oscillators in complex topologies: the choice of $\lambda_{i j}$. In principle, one should choose $\lambda_{i j}$ in a way that the model is well behaved in the thermodynamic limit $N \rightarrow$ $\infty$ or to avoid an obvious bias in the dynamics due to the system's size. For the fully connected graph these guidelines are easily satisfied by adopting $\lambda_{i j}=\lambda / N$, since in this topology the number of neighbors that an oscillator has grows linearly with $N$. Therefore, the normalization of the coupling strength in complex networks should be defined to incorporate the same scaling found in the dependence of the connectivity of the nodes on the system size. However, the problem in finding a suitable normalization resides in the fact that different network models have different scalings, making the definition of $\lambda_{i j}$ to be not unique. This fact led many researchers to simply adopt a homogeneous coupling as $\lambda_{i j}=\lambda \forall i, j$ yielding $(32,69)$

$$
\dot{\theta}_{i}=\omega_{i}+\lambda \sum_{j=1}^{N} A_{i j} \sin \left(\theta_{j}-\theta_{i}\right) .
$$

Establishing the interaction between the oscillators to be via a constant coupling without any further normalization seems to be more appropriate when comparing the synchronization properties of different networks given the different scaling arising from different topologies. Nevertheless, the search for an intensive coupling is indeed an important issue regarding the formulation and the analysis of the model in complex networks, specially concerning the determination of the critical coupling for the onset of synchronization. Arenas et al. (69) provide an interesting discussion about the different prescriptions for 
intensive couplings and also the corresponding consequences of each choice in the network dynamics. In this thesis we adopt the constant coupling formulation (2.23) unless another definition is explicitly stated.

By defining the local order parameter

$$
r_{i} e^{i \psi_{i}(t)}=\sum_{j=1}^{N} A_{i j} e^{i \theta_{j}(t)}
$$

it is possible to rewrite Eqs. 2.23 as

$$
\dot{\theta}_{i}=\omega_{i}+\lambda r_{i} \sin \left(\psi_{i}-\theta_{i}\right) .
$$

In contrast with the model in the fully connected graph, the decoupled equations are not expressed in terms of a common field equally felt by all oscillators, instead each oscillator feels an equivalent coupling that only accounts for the interaction with its immediate neighborhood. This brings us to one of the most employed approaches in the analytical treatment of dynamical processes in networks, namely the consideration of mean-field approximations (MFAs).(39) Such an approximation scheme is translated in the Kuramoto dynamics by supposing the existence of a global mean-field $r e^{i \psi}$ that relates with each $r_{i} e^{i \psi_{i}}$ through (32)

$$
r e^{i \psi}=\frac{1}{k_{i}} r_{j} e^{i \psi_{i}}=\frac{1}{k_{i}} \sum_{j=1}^{N} A_{i j} e^{i \theta_{j}} .
$$

The relation above basically states that the local mean-fields should be proportional to the global one weighted by the local connectivity, i.e. $r_{i}=r k_{i}$. This assumption is reasonable to be adopted only if the network under study has a sufficient large average degree with vanishing degree-degree correlations and without the presence of communities, i.e. groups of nodes that are more connected within themselves than with the rest of the network (69, 70) (see $(71,72)$ for a thorough study on the validity of MFAs for different dynamical processes in networks). Using Eq. 2.26, the phases evolution are decoupled as

$$
\dot{\theta}_{i}=\omega_{i}+\lambda r k_{i} \sin \left(\psi-\theta_{i}\right) .
$$

Note that the local topology of node $i$ is incorporated in its effective coupling via the degree $k_{i}$, in contrast with the fully connected graph. For this reason, the effects of the network topology on dynamics can be masked if the coupling strength in Eq. 2.22 is set as $\lambda_{i j}=\lambda / k_{i}$, where the normalization by the degree ends up neutralizing the heterogeneous interactions with the mean-field. It is worth mentioning that the MFA in Eq. 2.26 is equivalent to the so-called annealed network approximation.(23) In this approach, one replaces the adjacency matrix term $A_{i j}$ by the ensemble average $\tilde{A}_{i j}$ corresponding to the probability that two nodes with degrees $k_{i}$ and $k_{j}$ are connected in the configuration model, i.e.

$$
\tilde{A}_{i j}=\frac{k_{i} k_{j}}{N\langle k\rangle}
$$


yielding

$$
\dot{\theta}_{i}=\omega_{i}+\lambda k_{i} \sum_{j=1}^{N} \frac{k_{j}}{N\langle k\rangle} \sin \left(\theta_{j}-\theta_{i}\right) .
$$

In effect, the original complex network is mapped to a fully connected weighted graph whose new adjacency matrix $\tilde{A}_{i j}$ conserves the former degree sequence, i.e. $k_{i}=\sum_{j} A_{i j}=$ $\sum_{j} \tilde{A}_{i j}$, resembling in this way the original connectivity pattern. Furthermore, Eq. 2.29 motivates the following definition of the global order parameter:

$$
r e^{i \psi(t)}=\frac{1}{N\langle k\rangle} \sum_{j=1}^{N} k_{j} e^{i \theta_{j}(t)}
$$

which is equivalent to Eq. 2.26 and leads precisely to Eq. 2.27.

One of the main advantages of the approximation scheme of Eqs. 2.26 and 2.28 is that it straightforwardly enables a formulation of the model in the continuum limit which in turn allows further analytical tractability in the same spirit of Kuramoto's original approach. To this end, assume that $\rho(\theta, t \mid \omega, k)$ is the density of oscillators with phase $\theta$ at time $t$ with given natural frequency $\omega$ and degree $k$ satisfying the normalization condition

$$
\int_{0}^{2 \pi} \rho(\theta, t \mid \omega, k) d \theta=1
$$

Supposing that the dynamics takes place in a uncorrelated network with degree distribution $P(k)$, we have that the probability that a randomly selected edge has at its end a node with phase $\theta$ at time $t$ with frequency $\omega$ and degree $k$ is given by $(32,73)$

$$
\frac{k P(k)}{\langle k\rangle} g(\omega) \rho(\theta, t \mid \omega, k)
$$

The equations for the phase evolution in the continuum limit are then obtained by switching the sum in the right-hand side of Eq. 2.29 by the average using Eq. 2.32, i.e. $(32,73)$

$$
\dot{\theta}=\omega+\frac{\lambda k}{\langle k\rangle} \int d \omega^{\prime} \int d k^{\prime} \int d \theta^{\prime} g\left(\omega^{\prime}\right) P\left(k^{\prime}\right) k^{\prime} \rho\left(\theta^{\prime}, t \mid \omega^{\prime}, k^{\prime}\right) \sin \left(\theta^{\prime}-\theta\right),
$$

Essentially, all nodes in the same degree class are assumed to be dynamically equivalent and the network is no longer described by the adjacency matrix, instead the network structure is now abstracted in the degree distribution and in two-vertex degree correlations. Such a coarse-grain technique is often referred as heterogeneous mean-field theory (74, 75) and has been extensively employed in the study of several dynamical processes on networks.(39)

In order to decouple Eq. 2.33 one can define the global order parameter as

$$
r e^{i \psi(t)}=\frac{1}{\langle k\rangle} \int d \omega \int d k \int d \theta P(k) k g(\omega) \rho(\theta, t \mid \omega, k) e^{i \theta(t)}
$$


Note that Eq. 2.34 is exactly the continuum limit version of the order parameter introduced in the annealed network approximation (2.30). Writing Eq. 2.33 in terms of the order parameter in the continuum limit, distribution $\rho(\theta, t \mid \omega, k)$ should then obey the continuity equation

$$
\frac{\partial \rho(\theta, t \mid \omega, k)}{\partial t}=-\frac{\partial}{\partial \theta}\{\rho(\theta, t \mid \omega, k)[\omega+\lambda k r \sin (\psi-\theta)]\}
$$

whose solutions in the stationary region, assuming $\psi=0$ without loss of generality, are given by

$$
\rho(\theta \mid \omega, k)=\left\{\begin{array}{ll}
\delta\left[\theta-\arcsin \left(\frac{\omega}{\lambda k r}\right)\right] & \text { if }|\omega| \leq \lambda k r \\
\frac{C(\omega, k)}{|\omega-\lambda k r \sin \theta|} & \text { otherwise, }
\end{array},\right.
$$

where $C(\omega, k)=\sqrt{\omega^{2}-(\lambda k r)^{2}}$ and $\delta(\cdot)$ is the Dirac delta function. The first and second terms correspond to the density of synchronous and drifting oscillators. Substituting Eq. 2.36 in Eq. 2.34 one obtains

$$
\int d k P(k) k=\lambda \int d k P(k) k^{2} \int_{-1}^{1} d x g(x \lambda r k) \sqrt{1-x^{2}}
$$

which tending $r \rightarrow 0^{+}$leads to critical coupling for the onset of synchronization given by $(32,73)$

$$
\lambda_{c}=\lambda_{c}^{\mathrm{KM}} \frac{\langle k\rangle}{\left\langle k^{2}\right\rangle}
$$

This equation is one of the most known results related to the dynamics of $\mathrm{Ku}$ ramoto oscillators in networks. It asserts that the value of the critical coupling for the onset of synchronization in the fully connected graph is rescaled by the ratio $\langle k\rangle /\left\langle k^{2}\right\rangle$ of the first two moments of the degree distribution $P(k)$. Therefore, according to Eq. 2.38, the more heterogeneous the network, the weaker the coupling strength required to synchronize its oscillators. This highlights the role played by the hubs in network dynamics acting improving the overall collective behavior. Furthermore, the mean-field scheme gives a finite $\lambda_{c}$ for SF networks with $\gamma>3$ in the thermodynamic limit $N \rightarrow \infty$, in agreement with simulations of sufficient large networks. $(69,76)$ However, problems arise for more heterogeneous networks with $2<\gamma<3$. In principle, one would expect partial synchronization to emerge for any $\lambda>0$, since $\left\langle k^{2}\right\rangle \rightarrow \infty$ in this range of $\gamma$. However, as extensive simulations show (69), that seems to be not the case even if the finite number of nodes is taken into account in the estimation of $\lambda_{c}$ using Eq. 2.38. More specifically, for $\gamma=3$, the second moment of the degree distribution scales with the system size as $\left\langle k^{2}\right\rangle \sim \ln N$, leading to $\lambda_{c} \sim 1 / \ln N .(23,24,69)$ Although a very high number of oscillators is indeed a limiting factor, simulations with reasonably large networks already present discrepancies with this estimate of $\lambda_{c}$. Notwithstanding, evidences show that in fact $\lambda_{c}$ for SF networks with $2<\gamma<3$ seems to converge to a constant value as the system size $N$ is increased, in striking contrast with the prediction of the MFA.(32) Therefore, the question left is what 
is the source of the disagreement between the result predicted in Eq. 2.38 and the results observed in simulations. Much has been conjectured about this puzzle $(23,24,39,69)$ but the problem remains open.

\subsubsection{The second-order Kuramoto model}

The Kuramoto model was firstly conceived with the goal to qualitatively explain how populations of oscillators fall into synchronization. However, since its formulation more than 40 years ago many natural phenomena have been described in terms of the collective dynamics of phase oscillators, a fact that not even Kuramoto himself could foresee.(77) The development of the second-order Kuramoto has its roots in one of the most eye-catching examples of synchronization in nature - the flashing of fireflies.(2) Although first-order oscillators such as those in Eq. 2.12 can phenomenologically explain some aspects behind the onset of collective behavior in these systems, when compared to experimental data (78), one notices that the model predicts much faster time scales to reach the stationary state. Another drawback of the classical model (2.12) in describing fireflies resides in the fact that infinite couplings are required to fully synchronize the population, whereas some species indeed appear to be able to sustain perfect coherence under stimulus.(2) In principle, this could be remedied by considering the oscillators to be identical, i.e. $\omega_{i}=\omega_{0} \forall i$. While this scheme yields states in which $R=1$, it still leaves the time-scale problem; besides, assuming that fireflies flash with identical natural frequencies seems rather unrealistc. In 1991, Ermentrout (79) shed light into this problem by developing a phase oscillator model able to describe more accurately the synchronous firing. His idea was to allow an adaptive effect in the natural frequencies. Indeed, this mechanism is biologically sound; fireflies such as Pteroptix malaccae can in fact increase and decrease their intrisinc period of oscillation by amounts up to 15\%.(80) Inspired by this finding, Tanaka et al. $(30,31)$ introduced the sinusoidal coupling in the model in $(79)$ and adapted the original self-consistent analysis by Kuramoto to the inertial case. In this section, we will briefly discuss the main ideas behind the approach in $(30,31)$, which we will later in Chapter 4 generalize to heterogeneous topologies as part of our original contributions.

\subsubsection{Mean-field theory: Fully connected graph}

The second-order Kuramoto model is given by the following set of equations

$$
m \ddot{\theta}_{i}=-\alpha \dot{\theta}_{i}+\omega_{i}+\frac{\lambda}{N} \sum_{j=1}^{N} \sin \left(\theta_{j}-\theta_{i}\right), i=1, \ldots, N
$$

where $m$ is the inertia and $\alpha$ is the damping factor associated to the oscillators and again $\omega_{i}$ is the natural frequency of the $i$ th oscillator. For the sake of clarity we will assume 
(a)

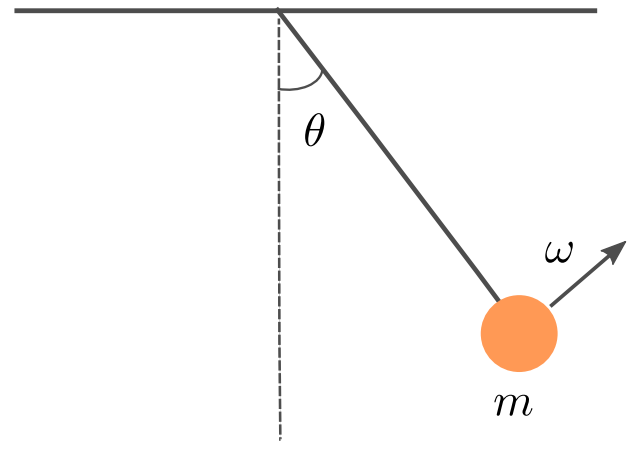

(b)

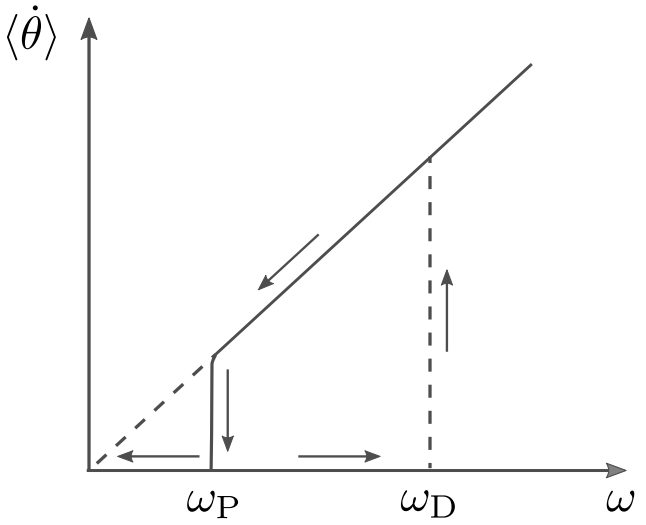

Figure 2 - (a) Illustration of a damped pendulum of mass $m$ subjected to a constant torque $\omega$. (b) Schematic diagram of $\omega \times\langle\dot{\theta}\rangle$.

Source: By the author.

in this section $\alpha=1$ without loss of generality. When stating this model the following question immediately crosses our minds: which changes in the collective dynamics of the first-order model could possibly arise if the inertial term is included? To answer this question, one could start by first writing Eq. 2.39 in terms of the mean-field quantities $R$ and $\psi$ as

$$
m \ddot{\theta}_{i}=-\dot{\theta}_{i}+\omega_{i}+\lambda R \sin \left(\psi-\theta_{i}\right),
$$

and then attempt to follow the same analysis as done by Kuramoto in his original papers $(14,15)$ and described in the beginning of Sec. 2.2. However, Eq. 2.40 is the same equation of damped driven pendulum, with $\omega_{i}$ playing the role of a constant torque applied to it. This fact already tells us a hint of the different dynamical aspects we can expect from the second-order model because the damped driven pendulum is known to exhibit hysteretic behavior in the plane $\left(\theta_{i}, \dot{\theta}_{i}\right)$. Precisely, suppose we have a pendulum of mass $m$ initially at rest subjected to a constant torque $\omega$. If we keep increasing $\omega$, the pendulum will start its movement only after a certain threshold $\omega_{\mathrm{D}}$ is crossed (see Fig. 2 for an illustration). On the other hand, if $\omega$ is now decreased, we will see that the system will continue to oscillate even for $\omega<\omega_{\mathrm{D}}$, being brought back to rest only when a certain torque value $\omega_{\mathrm{P}}$ is reached. Therefore, since the population of second-order Kuramoto oscillators (Eq. 2.39) can be seen as a collection of interacting pendulums, we then expect to observe some hysteretic behavior regarding the onset of synchronization in this system. Extending the thought experiment of the isolated pendulum to system (2.39) we foresee two possible scenarios: [I] starting in the asynchronous state with the whole population constituted by drifting oscillators $\left(R \approx 0\right.$ and $\left.\left|\left\langle\dot{\theta}_{i}\right\rangle\right|>0\right)$, as the coupling strength $\lambda$ is increased, the portion of oscillators with $\omega_{i}<\omega_{\mathrm{P}}(\lambda)$ will be locked $\left(\left|\left\langle\dot{\theta}_{i}\right\rangle\right|=0\right)$ and a giant synchronous component will start to be formed (see Fig. 2(b)). [II] In the opposite situation in which we initiate the system with a large $\lambda$ so that fully synchronized state 
is achieved $\left(R \approx 1\right.$ and $\left.\left|\left\langle\dot{\theta}_{i}\right\rangle\right|=0\right)$, if we decrease the coupling, the oscillators will then fall into incoherence as soon as $\omega>\omega_{\mathrm{D}}=\lambda r$ is satisfied, which is when the locked solution $\left(\ddot{\theta}_{i}=\dot{\theta}_{i}=0\right)$ ceases to be valid. Figure 4 shows a typical dependence of the order parameter $R$ as a function of $\lambda$ for a population of $N=10^{3}$ oscillators with Lorenztian frequency distribution as $g(\omega)=1 /\left[\pi\left(1+\omega^{2}\right)\right]$.

With this in mind one is able to develop a self-consistent analysis of the order parameter separately for cases I and II. Writing the total order parameter as $R^{\mathrm{I} \text { II }}=$ $R_{\text {lock }}^{\mathrm{I} \text { II }}+R_{\text {drift }}^{\mathrm{I} \text { II }}$, taking the continuum limit of (2.40), and following Sec. 2.2.1, we find that the contribution of the locked oscillators in the above discussed scenarios is given by $(30,31)$

$$
R_{\text {lock }}^{\mathrm{I}, \mathrm{II}}=\lambda R^{\mathrm{I}, \mathrm{II}} \int_{-\theta_{\mathrm{P}, \mathrm{D}}}^{\theta_{\mathrm{P}, \mathrm{D}}} \cos ^{2} \theta g\left(\lambda R^{\mathrm{I}, \mathrm{II}} \sin \theta\right) d \theta,
$$

where $\theta_{\mathrm{P}}=\arcsin \left[\omega_{\mathrm{P}} /\left(\lambda R^{\mathrm{I}}\right)\right]$ and $\theta_{D}=\arcsin \left[\omega_{\mathrm{D}} /\left(\lambda R^{\mathrm{II}}\right)\right]$. Analogously as in the first-order model, the contribution of the drifting oscillators follows $(30,31)$

$$
R_{\mathrm{drift}}^{\mathrm{IIII}}=\int_{|\omega|>\omega_{\mathrm{P}, \mathrm{D}}} \int e^{i \theta(t, \omega)} \rho_{\mathrm{drift}}(\theta, \omega) g(\omega) d \theta d \omega,
$$

where $\rho_{\text {drift }}(\theta, \omega)$ is the density of drifting oscillators with phase $\theta$ and frequency $\omega$. It turns out that $\rho_{\text {drift }}(\theta, \omega)$ cannot be determined as straightforwardly as in Sec. 2.2.1 due to the inertia term $m \ddot{\theta}$. A strategy to circumvent this problem is to switch the integral on $\theta$ by a temporal average over a period of oscillation, since $\rho_{\text {drift }}$ is a $2 \pi$-periodic function on $\theta .(31)$ More precisely, again as in Sec. 2.2.1, the density of drifting oscillators is still proportional to $\rho_{\text {drift }} \sim|\dot{\theta}|^{-1}$, which together with the normalization condition $\int_{0}^{2 \pi} \rho_{\text {drift }}(\theta, \omega) d \theta=$ $\int_{0}^{\tilde{T}} \rho_{\text {drift }}|\dot{\theta}| d t=1$ yields $(31)$

$$
\rho_{\text {drift }}(\theta, \omega)=\tilde{T}|\dot{\theta}|^{-1}=\frac{\tilde{\omega}}{2 \pi}|\dot{\theta}|^{-1},
$$

where $\tilde{T}$ and $\tilde{\omega}$ are the period and frequency of the drifting oscillators. Substituting the previous equation into Eq. 2.42 gives (31)

$$
R_{\text {drift }}^{\mathrm{I}, \mathrm{II}}=\frac{1}{2 \pi} \int_{|\omega|>\omega_{\mathrm{P}, \mathrm{D}}} \int_{0}^{\tilde{T}} e^{i \theta(t, \omega)} \tilde{\omega}|\dot{\theta}|^{-1} \dot{\theta} g(\omega) d t d \omega=\frac{1}{2 \pi} \int_{|\omega|>\omega_{\mathrm{P}, \mathrm{D}}} \int_{0}^{\tilde{T}} e^{i \theta(t, \omega)}|\tilde{\omega}| g(\omega) d t d \omega,
$$

where the following relations hold true: $\dot{\theta}>0$ for $\tilde{\omega}>0, \dot{\theta}<0$ for $\tilde{\omega}<0$, and $\tilde{\omega}(-\omega)=$ $-\tilde{\omega}(\omega)$. Considering $g(\omega)=g(-\omega)$, Eq. 2.44 is simplified to $(31)$

$$
R_{\mathrm{drift}}^{\mathrm{I}, \mathrm{II}}=\frac{1}{\pi} \int_{\omega>\omega_{\mathrm{P}, \mathrm{D}}} \int_{0}^{\tilde{T}} \cos \theta(t, \omega)|\tilde{\omega}| g(\omega) d t d \omega .
$$

Unfortunately, Eq. 2.45 offers no exact solution. Tanaka et al. (31) estimated the integral $\int_{0}^{\tilde{T}} \cos \theta(t, \omega) d t$ by employing the Poincaré-Lindstead method, whereby expansions up to $1 /(m \omega)^{3}$ were considered leading to (see Appendix A)

$$
R_{\mathrm{drift}}^{\mathrm{I}, \mathrm{II}} \simeq-m \lambda R \int_{\omega_{\mathrm{P}, \mathrm{D}}}^{\infty} \frac{1}{(m \omega)^{3}} g(\omega) d \omega .
$$




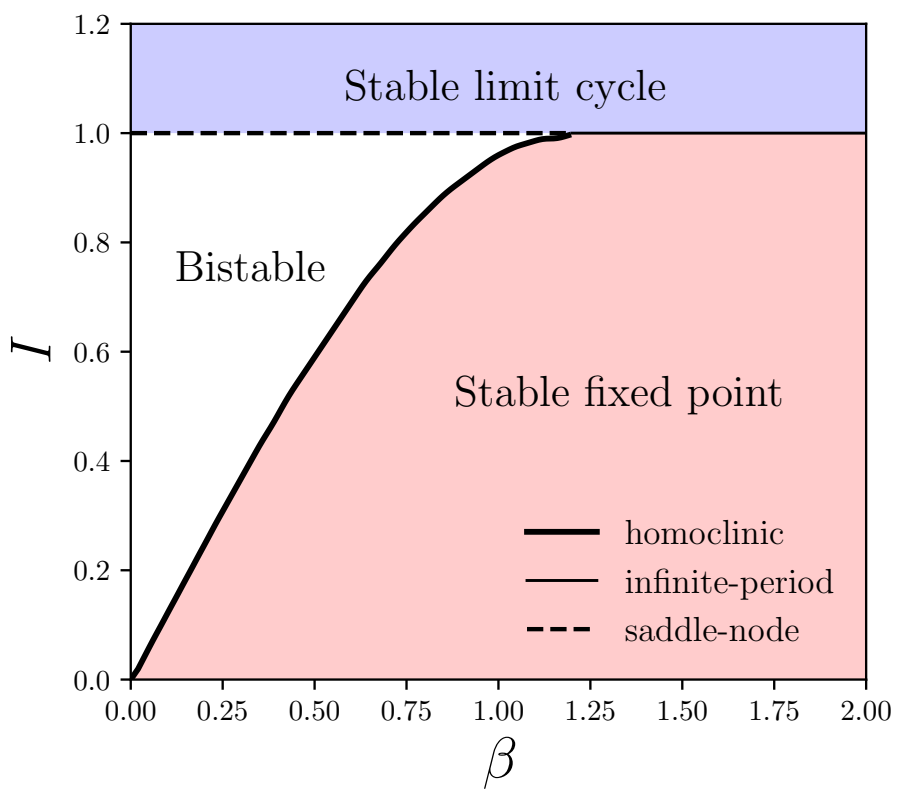

Figure 3 - Stability diagram of Eq. 2.47

Source: By the author.

The final task left to uncover the dependence of $R^{\mathrm{I} \text {,II }}$ on $\lambda$ is the determination of frequencies $\omega_{\mathrm{P}, \mathrm{D}}$. To this end, it is convenient to set the new time-scale $s=t \sqrt{\lambda R / m}$ and write Eq. 2.40 in the non-dimensional form as

$$
\frac{d^{2} \theta}{d s^{2}}+\beta \frac{d \theta}{d s}+\sin \theta=I
$$

from which we omitted the indexes $i$ and set $\psi=0$, without loss of generality. It turns out that Eq. 2.47 is the very same equation of a damped driven pendulum with effective damping coefficient $\beta=1 / \sqrt{\lambda R m}$ and subjected to the torque $I=\omega / \lambda R$. Formulated in this way, the problem of determining $\omega_{\mathrm{P}, \mathrm{D}}$ becomes straightforward since we know how the stability diagram of Eq. 2.47 looks like in terms of variables $\beta$ and $I$ (cf. the damped driven pendulum problem in (81) for an introductory text and (82) for a rigorous treatment). As shown in Fig. 3, for $I>1(\omega>\lambda R)$, system (2.47) operates in a stable limit cycle, meaning that the dynamics for this range of parameters is periodic. This region corresponds to the asynchronous regime due to the fact that for $\omega>\lambda R$ the locked solution of Eq. 2.40 is no longer valid. Supposing that $\beta \lesssim 1.2$, as $I$ is decreased a saddle-node bifurcation occurs at $I=1\left(\omega_{\mathrm{D}}=\lambda R\right)$ and the system enters in the region where the stable limit cycle and the stable fixed point solutions coexist in the phase space $\theta-\dot{\theta}$. Here, to which state system (2.47) will converge depends entirely on the initial conditions. At last, if torque $I$ is decreased even more, the stable limit cycle is destroyed in a homoclinic bifurcation and the only possible solution for Eq. 2.47 is the stable fixed point $\theta=\arcsin (\omega / \lambda R)$. By using the technique known as the Melnikov's method $(81,82)$, it is possible to show that 


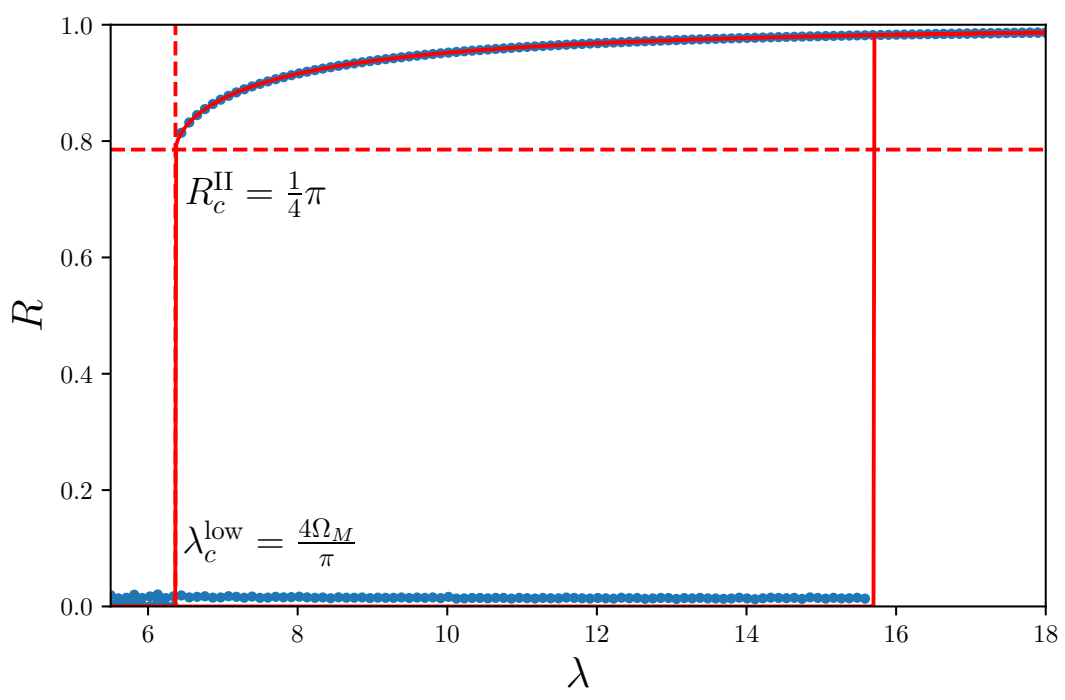

Figure 4-Hysteretic synchrony of the second-order Kuramoto model. Critical conditions (Eq. 2.55) for the backward propagation of the system are depicted in the plot. Dashed lines are used to highlight the critical point $\left(\lambda_{c}^{\text {low }}, R_{c}^{\mathrm{II}}\right)$ predicted by the theory. Theoretical solutions are derived by solving Eqs. 2.50-2.53. Dots are obtained by numerically evolving Eqs. 2.39 considering $N=10^{3}$ oscillators, $\alpha=1, m=1, \Omega_{\mathrm{M}}=5$ and $d t=0.01$. Order parameter $R$ is averaged over the time span $t \in[200,500]$.

Source: By the author.

this latter transition happens at $I \simeq 4 \beta / \pi$, which leads to (31)

$$
\omega_{\mathrm{P}} \simeq \frac{4}{\pi} \sqrt{\frac{\lambda R}{m}}
$$

Noteworthy, this sequence of transitions is only valid if the dimensionless damping $\beta$ is small enough; in the overdamped limit $(\beta \gtrsim 1.2$, see Fig. 3$)$ the stable limit cycle is annihilated by an infinite-period bifurcation. Finally, the total order parameter is thus obtained by summing the contributions of locked and drifting oscillators, i.e. $R^{\mathrm{I}, \mathrm{II}}=$ $R_{\text {locked }}^{\mathrm{I}, \mathrm{II}}+R_{\text {drift }}^{\mathrm{I}, \mathrm{II}}$.

Let us illustrate how the theory just described applies to the case of $N$ secondorder Kuramoto oscillators coupled in a fully connected graph with natural frequencies distributed according to the distribution

$$
g(\omega)= \begin{cases}\frac{1}{2 \Omega_{\mathrm{M}}} & , \text { for }|\omega| \leq \Omega_{\mathrm{M}} \\ 0 & , \text { for }|\omega|>\Omega_{\mathrm{M}} .\end{cases}
$$

We should keep in mind that frequencies $\omega_{\mathrm{P}}$ and $\omega_{\mathrm{D}}$ are directly related with the critical couplings $\lambda_{c}^{\text {up }}$ and $\lambda_{c}^{\text {low }}$ for the onset of synchronization associated to the increasing and decreasing coupling branches, respectively. In other words, in the scenario in which the coupling is adiabatically decreased, we assume that all oscillators are locked (fully 
synchronized state) and, as soon as $\omega_{i}>\omega_{D}$ holds for some $i$, the synchronous component will immediately start to fall apart due to the fact that some oscillators will have its fixed points converted into stable limit cycles. This transition happens precisely at $\lambda=\lambda_{c}^{\text {low }}$. On the other hand, imaging that population is initiated in complete incoherence, the synchronous component will be formed in the moment that the coupling strength is high enough to yield $\omega_{i}<\omega_{\mathrm{P}}$ for certain oscillators. The smaller coupling that leads to the latter inequality is then $\lambda=\lambda_{c}^{\text {up }}$. It is important remark that we have not assumed so far that the transitions between asynchronous and synchronous states are discontinuous, however we do expect to that the order parameter $R$ exhibits some abrupt dependence on $\lambda$ due to the analogy with the damped pendulum.

Calculating $R^{\mathrm{I}, \mathrm{II}}=R_{\text {locked }}^{\mathrm{I} \text { II }}+R_{\text {drift }}^{\mathrm{I} \text {,II }}$ using Eqs. 2.41 and 2.46 for the frequency distribution in Eq. 2.49 we obtain the following self-consistent equations: for increasing $\lambda$ $[\mathrm{I}]$, if $\omega_{\mathrm{P}}<\Omega_{\mathrm{M}}\left(\lambda<\lambda_{c}^{\mathrm{up}}\right), R^{\mathrm{I}}=R_{\text {locked }}^{\mathrm{I}}+R_{\text {drift }}^{\mathrm{I}}$ yields

$$
R^{\mathrm{I}}=\frac{\lambda R^{\mathrm{I}}}{2 \Omega_{\mathrm{M}}} \arcsin \left(\frac{\omega_{\mathrm{P}}}{\lambda R^{\mathrm{I}}}\right)+\sqrt{1-\left(\frac{\omega_{\mathrm{P}}}{\lambda R^{\mathrm{I}}}\right)^{2}}+\frac{\lambda R^{\mathrm{I}}}{2 \Omega_{\mathrm{M}}}\left(\frac{1}{\Omega_{\mathrm{M}}^{2}}-\frac{1}{\omega_{\mathrm{P}}^{2}}\right) .
$$

If $\omega_{\mathrm{P}}>\Omega_{\mathrm{M}}\left(\lambda>\lambda_{c}^{\text {up }}\right)$ summing Eqs. 2.41 and 2.46 leads to

$$
R^{\mathrm{I}}=\frac{\lambda R^{\mathrm{I}}}{2 \Omega_{\mathrm{M}}} \arcsin \left(\frac{\Omega_{\mathrm{M}}}{\lambda R^{\mathrm{I}}}\right)+\sqrt{1-\left(\frac{\Omega_{\mathrm{M}}}{\lambda R^{\mathrm{I}}}\right)^{2}} .
$$

For the decreasing $\lambda[\mathrm{II}]$, if $\omega_{\mathrm{D}}<\Omega_{\mathrm{M}}\left(\lambda<\lambda_{c}^{\text {low }}\right), R^{\mathrm{II}}=R_{\mathrm{lock}}^{\mathrm{II}}+R_{\mathrm{drift}}^{\mathrm{II}}$ reads

$$
R^{\mathrm{II}}=\frac{\pi}{4} \frac{\lambda R^{\mathrm{II}}}{\Omega_{\mathrm{M}}}+\frac{\lambda R^{\mathrm{II}}}{2 \Omega_{\mathrm{M}} m^{2}}\left(\frac{1}{\Omega_{\mathrm{M}}^{2}}-\frac{1}{\omega_{\mathrm{D}}^{2}}\right),
$$

and when $\omega_{\mathrm{D}}>\Omega_{\mathrm{M}}\left(\lambda>\lambda_{c}^{\text {low }}\right)$ we obtain

$$
R^{\mathrm{II}}=\frac{\lambda R^{\mathrm{II}}}{2 \Omega_{\mathrm{M}}} \arcsin \left(\frac{\Omega_{\mathrm{M}}}{\lambda R^{\mathrm{II}}}\right)+\sqrt{1-\left(\frac{\Omega_{\mathrm{M}}}{\lambda R^{\mathrm{II}}}\right)^{2}} .
$$

Observe that Eqs. 2.51 and 2.53 are identical, with the detail that the former is valid for $\lambda>\lambda_{c}^{\text {up }}$, while the latter is defined in the range $\lambda>\lambda_{c}^{\text {low }}$. The full dependence of $R^{\mathrm{I}, \mathrm{II}}$ can then be uncovered by self-consistently solving Eqs. 2.50, 2.51, 2.52 and 2.53. It is also instructive to determine the critical points of the curves $R^{\mathrm{I}, \mathrm{II}}$. For decreasing $\lambda$, at $\lambda=\lambda_{c}^{\text {low }}$ we have that

$$
\omega_{\mathrm{D}}=\lambda_{c}^{\mathrm{low}} R_{c}^{\mathrm{II}}=\Omega_{\mathrm{M}}
$$

where $R_{c}^{\mathrm{II}}$ is the order parameter at the transition point $\lambda=\lambda_{c}^{\text {low }}$. Inserting Eq. 2.54 in Eq. 2.53 we obtain

$$
\lambda_{c}^{\text {low }}=\frac{4 \Omega_{\mathrm{M}}}{\pi} \text { and } R_{c}^{\mathrm{II}}=\frac{\pi}{4} .
$$

Note that for $\lambda<\lambda_{c}^{\text {low }}$ the solution of Eq. 2.52 yields $R^{\mathrm{II}}=0$, but at $\lambda=\lambda_{c}^{\text {low }}$, the order parameter $R^{\mathrm{II}}$ jumps discontinuously from 0 to $R_{c}^{\mathrm{II}}$. This result shows that the transition 
from the synchronous to the asynchronous state is of first-order type. Furthermore, the critical coupling is reached when $\omega_{\mathrm{D}}=\Omega_{\mathrm{M}}$, this means that the whole population becomes locked with mean-field in this point. Similarly, for increasing $\lambda$, at the critical coupling $\lambda_{c}^{\text {up }}$ we have $\omega_{\mathrm{P}}=\Omega_{\mathrm{M}}$, leading to the relation

$$
\lambda_{c}^{\mathrm{up}} R_{c}^{\mathrm{I}}=\frac{\pi^{2} \Omega_{\mathrm{M}} m}{16} .
$$

Substituting the previous equation in Eq. 2.51 we obtain

$$
R_{c}^{\mathrm{I}}=\frac{m \Omega_{\mathrm{M}} \pi^{2}}{32} \arcsin \left(\frac{16}{m \Omega_{\mathrm{M}} \pi^{2}}\right)+\frac{1}{2} \sqrt{1-\left(\frac{16}{m \Omega_{\mathrm{M}} \pi^{2}}\right)^{2}},
$$

which also gives $\lambda_{c}^{\text {up }}$ with Eq. 2.56. In Fig. 4 we compare these theoretical results with simulations considering $\Omega_{\mathrm{M}}=5$.

The theoretical framework introduced here for inertial phase oscillators sets the scene for Chapter 4, where we will extend the treatment developed in $(30,31)$ for oscillators in uncorrelated networks. 


\section{Synchronization of networks with non- vanishing transitivity}

In Sec. 2.1.2 we saw that during the last decade or so much effort has been put in the characterization and modeling of the structure of complex networks. As a part of this effort, we described the four major network models that boosted great part of the early theoretical developments and established the foundations of network science. We also saw that each model is able to mimic specific properties present in real-world networks such as low average shortest path lengths, heterogeneity of the degree distribution, high clustering, and so on. However, the modeling of these features by the classical models is somewhat limited in the sense that, while a given property of interest is accurately captured, many others are left aside. Let us briefly recall these limitations. Despite its simplicity, the ER model does reproduce a typical property from some real-world networks, namely low values for the average shortest path length or, in other words, the small-world phenomenon. However, as the networks become larger, the clustering coefficient quickly goes to zero. Another severe limitation is that the model only creates networks with Poisson degree distributions. This latter drawback is easily circumvented by the configuration model, whereby a network with any degree distribution can be created. However, it also suffers from the same lack of clustering as $N \rightarrow \infty$. The WS model, on the other hand, enables the construction of networks with realistic values of clustering and shortest path lengths. Indeed, this is the precise feature that made the model so successful in the complex systems literature. Unfortunately, although many real-world networks exhibit clustered structures jointly with low average shortest path lengths, the degree distributions of the yielded networks are way too narrow when compared to real data. In fact, shortly after the publication of the celebrated work by Watts and Strogatz, one witnessed a myriad of papers reporting the highly heterogeneous structure of networks with power-law degree distributions. These empirical observations motivated then the formulation of the BA model, which also belongs to the class of networks with locally tree-like topology in the thermodynamic limit.

Naturally, the vast majority of the early studies on synchronization phenomena in networks considered the aforementioned models as the substrate for the dynamics. However, evidently several higher-order topological properties found in real-world networks are overlooked by these mechanisms of network construction. In fact, triadic connections - which can be viewed as one of the simplest motifs ubiquitous in these real systems only emerge in random models as a by-product of the finite size of the generated networks.(18) Therefore, a clear understanding of how triangles affect the synchronization of 
coupled phase oscillators is still missing. In this chapter we give a first step towards filling this gap by extending the mean-field calculations presented in Sec. 2.2.1 to networks with non-vanishing transitivity generated by an analytically tractable model proposed independently by Newman and Miller $(60,83)$. We should mention though, that before the publication of $(60,83)$, the lack of a suitable random model with tunable clustering did not stop researchers to tackle the impact of clustering on the collective dynamics of oscillatory networks with alternative approaches. Before presenting our original results in Sec. 3.3 and 3.4, we will briefly review previous investigations on this topic (Sec. 3.1) and thereafter, in Sec. 3.2, we describe the model of Refs. $(60,83)$. This chapter is based on publications $(32,84)$ whose content will be closely followed in the next sections.

\subsection{Early approaches on dynamics of clustered networks}

One of the first approaches aimed at evaluating the impact of triangles on the dynamics of phase oscillators is given by McGraw and Menzinger (85-87). In order to precisely compare the coherence of clustered networks with that of non-clustered networks, the authors adopted the stochastic rewiring algorithm proposed by Kim (88). It consists of randomly selecting two edges and rewiring the connection of the associated nodes, accepting the new configuration in case the number of triangles in the network is increased. This procedure is then successively repeated until the desired transitivity is reached. The key feature of this process is that the degree sequence and, consequently, the degree distribution of the original network remain unchanged.(88) By applying this methodology to compare clustered with non-clustered networks, it was found that the coherence between oscillators is generally suppressed if the number of triangles is increased, regardless of whether the network connections are Poisson or power-law distributed.(85-87) Yet, clustered SF topologies exhibit an interesting behavior that is absent in ER networks. Namely, in clustered SF networks, the synchronization at low values of the coupling strength is enhanced when compared with unclustered networks with the same degree distribution.(85-87) The same effect of clustering on the onset of synchronization in SF networks was reported in (89). Evidence is shown that this particular behavior of the order parameter as a function of the coupling strength in clustered networks may be related to the following effect: in the process of stochastically rewiring the connections, not only the clustering of the networks is being changed, but also other properties, including the average shortest path length and the network modularity. In particular, changes in the local connections in order to increase the number of triangles contribute to increase the topological distance between the nodes and, in some cases, yield different communities. Thus, for weak couplings, partial synchronization is favored by these newly formed local connections, while higher couplings are required to overcome the long distances so that 
$r \sim 1$ is reached. $(85-87,89)$

These findings do not only show how the network dynamics is influenced by properties not encountered in traditional random models, but also demonstrate how difficult it is to disentangle the impact of different topological properties. More specifically, as previously mentioned, although the degree distribution of the networks is preserved, other network properties are modified along with the increasing of cycles of order three. In particular, the algorithm in (88) is known to strongly change the network assortativity and, in some cases, to induce the emergence of communities. $(85-87,90,91)$ Therefore, the influence of triangles on network synchronization is hard to be distinguished from these side effects generated by stochastic rewiring algorithms. Another limitation of using stochastic rewiring models to generate clustered networks resides in their analytic intractability, limiting the approaches to numerical calculations.

Seeking to overcome these difficulties and untangle the effects of triangles from other topological properties, in the next section we present a class of random graph model proposed in $(60,83)$ that generates networks with different levels of clustering while keeping degree-degree correlations close to zero. This model is the starting point for our original study in Sec. 3.3 on Kuramoto oscillators on clustered random networks.

\subsection{Configuration model for clustered networks - The Newman- Miller model}

The model proposed independently by Newman and Miller can be viewed as an extension of the standard configuration model. In the latter, one specifies first the desired degree distribution $P(k)$ from which a degree sequence $\left\{k_{i}\right\}(i=1, \ldots, N)$ is drawn dictating how many connections each node should have. The network is then created by randomly matching the half-edges (or stubs) in order to form complete edges. The process of network creation in the Newman-Miller is quite similar, but with the difference that now we need to specify two independent degree sequences, one accounting for the number of single-edges (edges that do not participate in a triangle) and a new sequence that specifies how many triangles are attached to each node. Let $s_{i}$ and $t_{\triangle i}$ be the number of single-edges and triangles attached to node $i$, respectively. Given the joint degree sequence $\left\{s_{i}, t_{\triangle i}\right\}(i=1, \ldots, N)$ one then creates the network by joining the stubs so that the number of single-edges and triangles initially set to each node is satisfied (Fig. 5 shows an example of an assignment of single edges and triangles). A simple way to construct a network realization in this model is given by the following method: (i) First create an independent single-edge stub list and a triangle stub list. (ii) Place the label of node $i$ in the first list $s_{i}$ times and in the second list $t_{\triangle i}$ times. Shuffle the two lists. (iii) The 


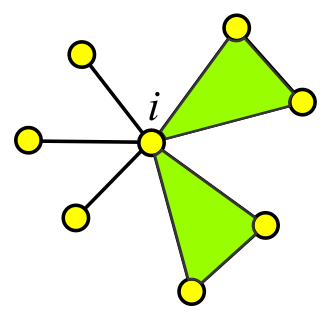

Figure 5 - Example of a node attached to three single edges and two triangles with $k_{i}=s_{i}+$ $2 t_{\triangle i}=7$. Therefore, the $i$ th element of the joint degree sequence is $\{3,2\}$.

Source: By the author.

network connections are then created by joining the pair of nodes in position $2 n$ and $2 n+1$ in the single-edge stub list and joining the nodes in positions $3 n, 3 n+1$, and $3 n+2$ in the triangle stub list. Self-loops and multiple edges may arise using this approach, however the probability of their occurrence vanishes as $N \rightarrow \infty$.(83)

Let $P\left(s, t_{\triangle}\right)$ be the joint probability distribution of nodes with $s$ single edges and $t_{\triangle}$. The traditional degree of a node in terms of $s$ and $t_{\triangle}$ is obtained as $k=s+2 t_{\triangle}$, since a triangle contributes with 2 to the total degree. Thus, the traditional degree distribution $P(k)$ is related to $P\left(s, t_{\triangle}\right)$ through $(60)$

$$
P(k)=\sum_{s, t_{\triangle}=0}^{\infty} P\left(s, t_{\triangle}\right) \delta_{k, s+2 t_{\triangle}} .
$$

The network transitivity can be straightforwardly calculated using the distributions $P\left(s, t_{\triangle}\right)$ and $P(k)$ by noticing that the total number of triangles in the network is given by $N_{\triangle}=$ $\frac{1}{3} N \sum_{s, t_{\Delta}} t_{\triangle} P\left(s, t_{\triangle}\right)$ while the number of connected triples reads $N_{3}=N \sum_{k}\left(\begin{array}{l}k \\ 2\end{array}\right) P(k)$. Substituting these quantities in Eq. 2.6 we obtain

$$
\mathcal{T}=\frac{\sum_{s, t_{\triangle}} t_{\triangle} P\left(s, t_{\triangle}\right)}{\sum_{k}\left(\begin{array}{l}
k \\
2
\end{array}\right) P(k)} .
$$

Note that the terms of $N$ are cancelled letting $\mathcal{T}$ to be nonzero in the limit of large networks $(N \rightarrow \infty)$, in contrast with the standard configuration model and the other models presented in Sec. 2.1.2.

Finally, by varying the number of triangles attached to each node, ensembles of networks with different levels of transitivity can be generated in order to assess the influence that higher clustering might have on the global dynamics of coupled phase oscillators.

\subsection{Mean-field theory for clustered networks}

To formulate the equations of motion in the continuum limit on clustered networks we should note that in the model by Newman and Miller the links should be distinguished 
between single edges and edges that participate in a triangle. Each node is then characterized by a pair $\left(s, t_{\triangle}\right)$ indicating how many edges from each type is attached to it. Using the joint degree distribution $P\left(s, t_{\triangle}\right)$ defined from the joint degree sequence $\left\{s_{i}, t_{\triangle i}\right\}$, we obtain the probability of randomly selecting an edge which is attached to an oscillator with phase $\theta$ at time $t$ for a given frequency $\omega$ and with $s$ single edges and $t_{\triangle}$ triangles:

$$
\frac{\left(s+2 t_{\triangle}\right)}{\langle k\rangle} P\left(s, t_{\triangle}\right) g(\omega) \rho\left(\theta, t \mid \omega, s, t_{\triangle}\right),
$$

where, analogously to Eq. 2.32, $\rho\left(\theta, t \mid \omega, s, t_{\triangle}\right)$ is the density of oscillators defined in terms of single edges and triangles. Using Eq. 3.3, the phases evolution read

$$
\begin{aligned}
\dot{\theta}= & \omega+\frac{\lambda\left(s+2 t_{\triangle}\right)}{\langle k\rangle} \int d \omega^{\prime} \int d s^{\prime} \int d t_{\triangle}^{\prime} \int d \theta^{\prime} g\left(\omega^{\prime}\right) P\left(s^{\prime}, t_{\triangle}^{\prime}\right) \\
& \times\left(s^{\prime}+2 t_{\triangle}^{\prime}\right) \rho\left(\theta^{\prime}, t \mid \omega^{\prime}, s^{\prime}, t_{\triangle}^{\prime}\right) \sin \left(\theta^{\prime}-\theta\right) .
\end{aligned}
$$

The global mean-field can also be redefined in order to account the contribution of single edge and triangles:

$$
r e^{i \psi}=\frac{1}{\langle k\rangle} \int d \omega \int d s \int d t_{\triangle} \int d \theta g(\omega) P\left(s, t_{\triangle}\right)\left(s+2 t_{\triangle}\right) \rho\left(\theta, t \mid \omega, s, t_{\triangle}\right) e^{i \theta},
$$

which enables Eq. 3.4 to be rewritten as

$$
\dot{\theta}=\omega+\lambda\left(s+2 t_{\triangle}\right) r \sin (\psi-\theta) .
$$

Following Sec. 2.2.1, $\rho\left(\theta, t \mid \omega, s, t_{\triangle}\right)$ will satisfy

$$
\frac{\partial \rho\left(\theta, t \mid \omega, s, t_{\triangle}\right)}{\partial t}=-\frac{\partial}{\partial \theta}\left\{\rho\left(\theta, t \mid \omega, s, t_{\triangle}\right)\left[\omega+\lambda\left(s+2 t_{\triangle}\right) r \sin (\psi-\theta)\right]\right\},
$$

whose time-independent solutions are given by

$$
\rho\left(\theta \mid \omega, s, t_{\triangle}\right)= \begin{cases}\delta\left[\theta-\arcsin \left(\frac{\omega}{\lambda\left(s+2 t_{\triangle}\right) r}\right)\right] & \text { if }|\omega| \leq \lambda\left(s+2 t_{\triangle}\right) r \\ \frac{C\left(\omega, s, t_{\triangle}\right)}{\left|\omega-\lambda\left(s+2 t_{\triangle}\right) r \sin \theta\right|} & \text { otherwise. }\end{cases}
$$

Considering $g(\omega)=g(-\omega)$ we have that only the locked solution of Eq. 3.8 will contribute to the order parameter, i.e.

$$
\langle k\rangle r=\int d \omega \int d s \int d t_{\triangle} \int d \theta g(\omega)\left(s+2 t_{\triangle}\right) P\left(s, t_{\triangle}\right) \sqrt{1-\left[\frac{\omega}{\lambda\left(s+2 t_{\triangle}\right) r}\right]^{2}} .
$$

Changing the variables to $\omega^{\prime}=\omega /\left(s+2 t_{\triangle}\right) \lambda r$ and considering $g(\omega)=(\sqrt{2 \pi})^{-1} e^{-\omega^{2} / 2}$, we obtain the following implicit equation for the order parameter $r$ (84)

$$
\begin{aligned}
\lambda= & \sqrt{\frac{8}{\pi}}\langle k\rangle\left\{\int d s \int d t_{\triangle} P\left(s, t_{\triangle}\right)\left(s+2 t_{\triangle}\right)^{2} e^{-\lambda\left(s+2 t_{\triangle}\right)^{2} r^{2} / 4}\right. \\
& \left.\times\left[I_{0}\left(\frac{\lambda^{2}\left(s+2 t_{\triangle}\right)^{2} r^{2}}{4}\right)+I_{1}\left(\frac{\lambda^{2}\left(s+2 t_{\triangle}\right)^{2} r^{2}}{4}\right)\right]\right\}^{-1}
\end{aligned}
$$



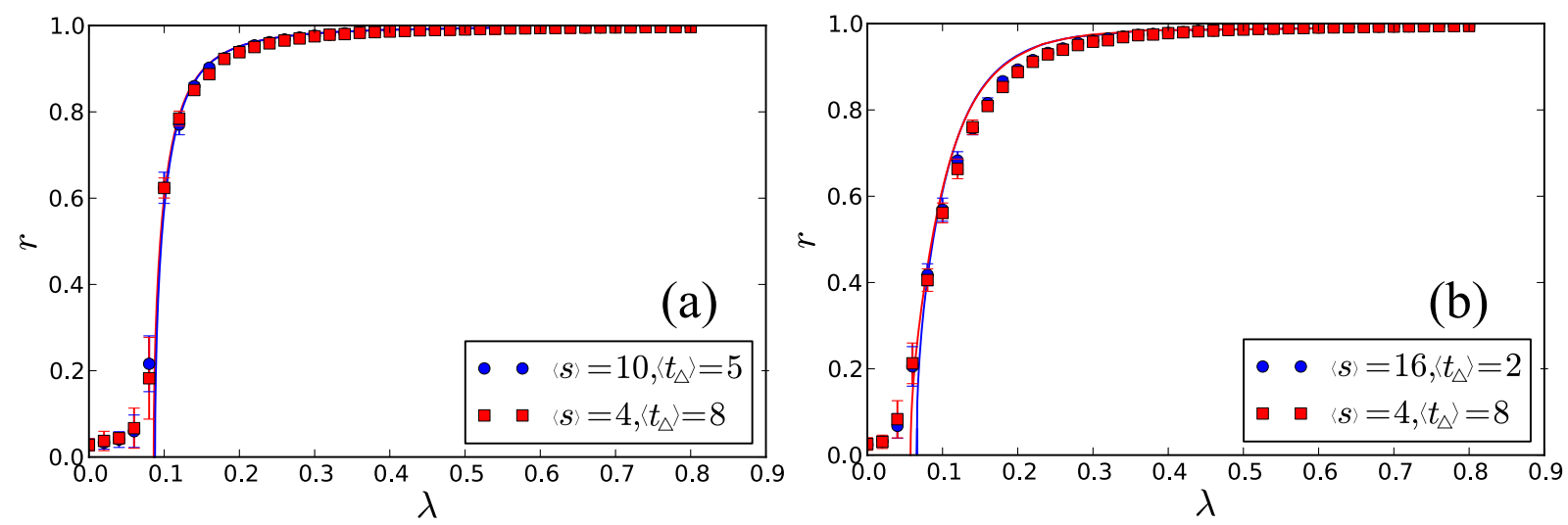

Figure 6 - Synchronization diagram for networks constructed with the configuration model for clustered networks considering (a) $P\left(s, t_{\triangle}\right)$ as a double Poisson degree distribution (Eq. 3.12) and (b) double SF degree distribution $P\left(s, t_{\triangle}\right) \propto s^{-\gamma} t_{\triangle}^{-\gamma}$, where $\gamma=3$. Lines are obtained by numerically solving Eq. 3.10 for different values of $\lambda$ and dots are obtained numerically evolving Eqs. 2.23. Each point is an average over 10 different realizations. Other parameters: $N=10^{3}$ and $\langle k\rangle=20$.

Source: Adapted from PERON; RODRIGUES; KURTHS. (84)

where $I_{0}$ and $I_{1}$ are the modified Bessel functions of first kind. Thus, tending $r \rightarrow 0^{+}$, we obtain the critical coupling $\lambda_{c}$ for the onset of synchronization

$$
\lambda_{c}=\sqrt{\frac{8}{\pi}}\langle k\rangle\left\{\int d s \int d t_{\triangle}\left(s+2 t_{\triangle}\right)^{2} P\left(s, t_{\triangle}\right)\right\}^{-1} .
$$

Note that the previous equation is similar to the corresponding result for unclustered networks, i.e. in both cases $\lambda_{c}$ is proportional to the ratio of the moments of the degree distribution. In the absence of triangles $\left(t_{\triangle}=0\right)$ we recover $\lambda_{c}=\sqrt{8 / \pi}\langle k\rangle /\left\langle k^{2}\right\rangle$, where the degrees are just due to single edges, $k=s$.

Equation 3.10 provides us a suitable way to analyze the network collective dynamics under different distribution of single edges and triangles. For a given fixed coupling $\lambda_{0}$, one seeks which value $r_{0}$ satisfies the equality 3.10 , obtaining in this way the pair $\left(\lambda_{0}, r_{0}\right)$. Repeating this procedure for several values of $\lambda_{0}$, we obtain the dependence $r=r(\lambda)$. Finally, the impact of clustering can be assessed by contrasting the outcomes of Eq. 3.10 for different choices of $P\left(s, t_{\triangle}\right)$. In the next section we compare these analytical results with numerical experiments.

\subsection{Numerical simulations}

All simulations performed in this section consider networks constructed using the configuration model presented in Sec. 3.2. For the sake of simplicity we will restrict the analysis to networks with independent distributions of single edges and triangles, i.e. 

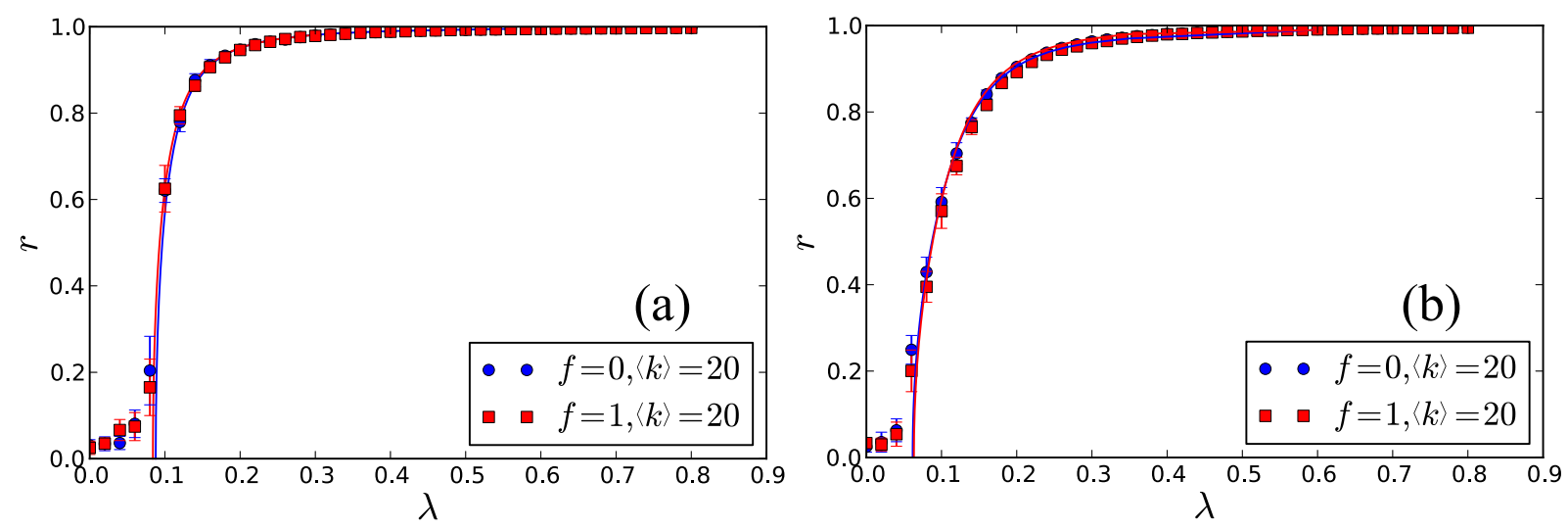

Figure 7 - Synchronization diagrams calculated as in Fig. 6 for random networks with degree distribution (a) $P(k)=e^{-\langle k\rangle}\langle k\rangle^{k} / k$ !, (b) $P(k) \propto k^{-\gamma}$, where $\gamma=3$; and joint degree distribution generated by Eq. 3.14. Each point is an average over ten network realizations. Solid lines correspond to the theoretical prediction from Eq. 3.10.

Source: Adapted from PERON; RODRIGUES; KURTHS. (84)

networks whose joint degree distributions satisfy $P\left(s, t_{\triangle}\right)=P(s) P\left(t_{\triangle}\right)$. Furthermore, as in the previous section, the natural frequency distribution adopted in the simulations is given by $g(\omega)=(\sqrt{2 \pi})^{-1} e^{-\omega^{2} / 2}$.

Let us start investigating the dynamics of networks with the double Poisson distribution of single edges and triangles:

$$
P\left(s, t_{\triangle}\right)=e^{-\langle s\rangle} \frac{\langle s\rangle^{s}}{s !} e^{-\left\langle t_{\triangle}\right\rangle} \frac{\left\langle t_{\triangle}\right\rangle^{t_{\Delta}}}{t_{\triangle} !} .
$$

In order to systematically investigate the dependence of the order parameter on the number of triangles, we keep the average degree $\langle k\rangle=\langle s\rangle+2\left\langle t_{\triangle}\right\rangle$ fixed and vary $\langle s\rangle$ and $\left\langle t_{\triangle}\right\rangle$. Figure 6(a) shows the synchronization diagram of networks with joint degree distribution given by Eq. 3.12 with $\langle k\rangle=20$. Interestingly, no significant difference is observed in the coherence among the oscillators as the number of triangles is increased. A similar behavior is observed in networks with more heterogeneous degree distributions. Precisely, Fig. 6(b) depicts the dependence of $r$ on $\lambda$ for networks with double power-law distribution given by

$$
P\left(s, t_{\triangle}\right) \propto s^{-\gamma} t_{\triangle}^{-\gamma}
$$

where $\gamma=3$. As we can see, despite the slight difference in the theoretical estimate of $\lambda_{c}$, networks with $\left\langle t_{\triangle}\right\rangle=8$ show statistically the same values of global synchronization as the ones with $\left\langle t_{\triangle}\right\rangle=2$. Noteworthy, the enhancement of coherence at low coupling strengths reported in $(85-87,89)$ for clustered SF networks generated via stochastic rewiring algorithms is absent here.

The results above are based on networks constructed via the setting of two degree sequences, $\left\{s_{i}\right\}$ and $\left\{t_{\triangle i}\right\}$, under the constraint of having a fixed total average degree 
$\langle k\rangle$. However, instead of controlling $\langle s\rangle$ and $\left\langle t_{\triangle}\right\rangle$ by changing the corresponding degree sequences, one can choose beforehand a given degree distribution $P(k)$ and then derive the joint degree distribution $P\left(s, t_{\triangle}\right)$ from it. One way to accomplish this is through the relation $(84,92)$

$$
P\left(s, t_{\triangle}\right)=P(k) \delta_{k, s+2 t_{\triangle}}\left[(1-f) \delta_{t_{\triangle}, 0}+f \delta_{t_{\triangle},\left\lfloor\left(s+2 t_{\triangle}\right) / 2\right\rfloor}\right],
$$

where $f \in[0,1]$ and $\lfloor\cdot\rfloor$ is the floor function. In this definition, we obtain $P\left(s, t_{\triangle}\right)$ by first specifying the total degree distribution $P(k)$ so that a fraction $f$ of the nodes has the maximum possible number of triangles, $k=\left\lfloor\left(s+2 t_{\triangle}\right) / 2\right\rfloor$, while the rest of the nodes is only attached to single edges $\left(t_{\triangle}=0\right)$. Substituting Eq. 3.14 into Eq. 2.6 we obtain the network transitivity $(84,92)$

$$
\mathcal{T}=f \frac{\sum_{k} k(P(2 k)+P(2 k+1))}{\sum_{k}\left(\begin{array}{c}
k \\
2
\end{array}\right) P(k)} .
$$

Equation 3.15 establishes a linear relationship between $\mathcal{T}$ and $f$; with $f=0$ we construct a network with a vanishing number of triangles, whereas for $f=1$ a network with the maximum value of $\mathcal{T}$ for a given $P(k)$ is obtained. Thus, this method enables the study of the extreme cases of the topology by controlling a single parameter in the network model while keeping $P(k)$ fixed. In this way, any change in the dependence $r=r(\lambda)$ can then be attributed to the variation in the number of triangles.

Figures $7(\mathrm{a})$ and (b) show the synchronization diagram for networks with $P(k)=$ $e^{-\langle k\rangle}\langle k\rangle^{k} / k$ ! and $P(k) \propto k^{-\gamma}$, respectively, with the corresponding joint distributions $P\left(s, t_{\triangle}\right)$ defined according to Eq. 3.14. For both degree distributions, no impact on the onset of coherent behavior is observed when the network has all its single edges converted to edges that participate in triangles $(f=1)$. This suggests that such motifs do not play an important role in the network synchronization. In other words, the dynamics of the clustered networks considered here can be suitably described by mean-field calculations developed for networks with a locally tree-like structure.(84) Interestingly, this innocuous effect of triangles on dynamical properties is not exclusive to networks of phase oscillators, but it has also been reported in other contexts such as in bond percolation, epidemic spreading and in the Ising model in scale-free networks. $(32,71,93,94)$

It is also worth dedicating some attention to the finite-size effects observed in the dependence of $r$ on $\lambda$ in the simulations of Figs. 6 and 7. As seen in the figures, the order parameter $r$ exhibits non-null values in coupling ranges where no synchronization is expected to emerge. In order to verify whether such a phenomenon is indeed an artefact due to the finite number of nodes, here we also perform simulations with larger networks with $P\left(s, t_{\triangle}\right)$ given by Eq. 3.14 considering $f=1$. Figure $8($ a) shows the synchronization diagram for networks with Poisson degree distribution $P(k)$ in Eq. 3.14 and $\langle k\rangle=20$ considering different sizes $N$. Note that the larger the networks, the smaller the values 

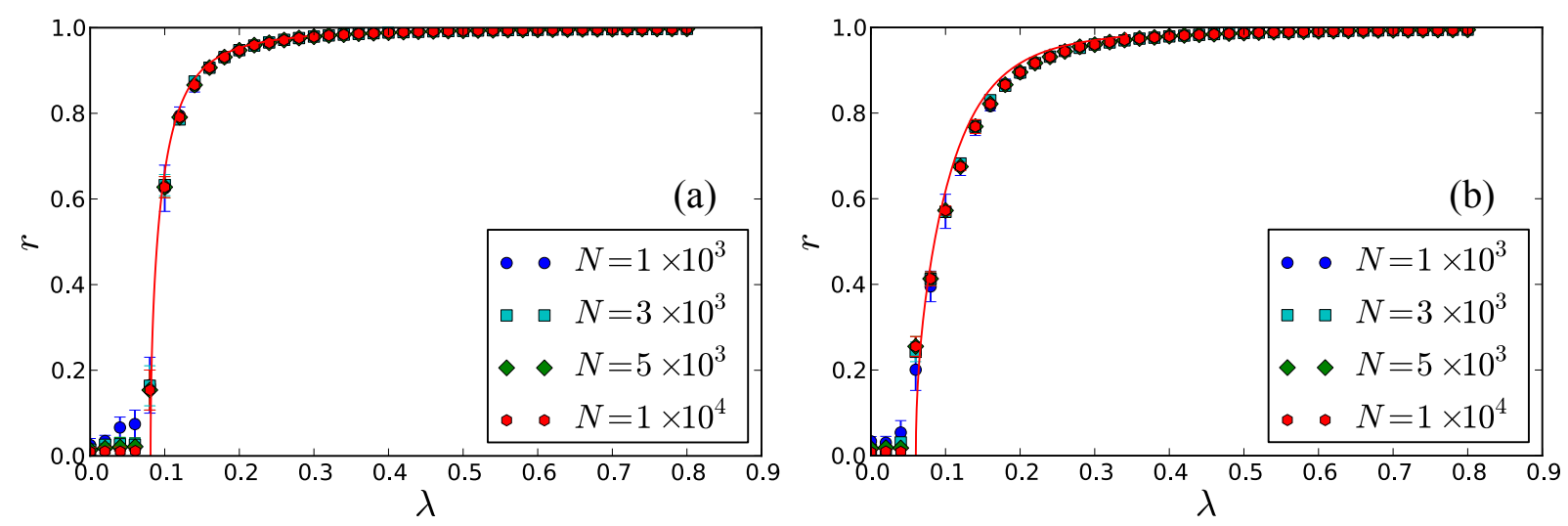

Figure 8 - Synchronization diagrams calculated as in the other figures for random networks with degree distribution (a) $P(k)=e^{-\langle k\rangle}\langle k\rangle^{k} / k$ !, (b) $P(k) \propto k^{-\gamma}$ with $\gamma=3$, and joint degree distribution generated by Eq. 3.14 with $f=1$. Each point is an average over ten network realizations. Solid lines correspond to the theoretical prediction from Eq. 3.10.

Source: Adapted from PERON; RODRIGUES; KURTHS. (84)

of $r$ in the region $\lambda<\lambda_{c}$. The same behavior is reported for clustered SF networks in Fig. 8(b). Similar results were obtained for unclustered networks (not shown here). For an exhaustive review on the finite-size scaling of $r$ and its dependence on the network topology, frequency assignment and sample-to-sample fluctuations, we refer the reader to $(32)$.

\subsection{Remarks on the effects of triangles in the Newman-Miller model}

Although the results of Secs. 3.3 and 3.4 contribute with more evidences that cycles of order three do not play an important role in network dynamics, it is important to specify the limitations of the configuration model with non-vanishing clustering to evaluate the contributions of triangles on dynamics. The model in $(60,83)$ is limited to generate networks in the so-called low-clustering regime in which the network transitivity has an upper bound $\mathcal{T}_{\max }=1 /(\langle k\rangle-1) .(95,96)$ The reason for this bound resides in the fact that the model does not allow the creation of overlapping triangles. In other words, a given edge is restricted to participate in a single triangle, limiting the clustering coefficient of a node with degree $k$ to $c(k) \leq 1 /(k-1)$. Moreover, the configuration model for clustered networks is not completely independent from effects of degree-degree correlations. It is possible to show that the assortativity $\mathcal{A}$ (see Sec. 2.1.1 for definition) of the model in $(60,83)$ as a function of $\mathcal{T}$ is given by (97)

$$
\mathcal{A}=\frac{\mathcal{T}-\mathcal{T}^{2}-\langle k\rangle \mathcal{T}^{2}}{1-\mathcal{T}+\langle k\rangle \mathcal{T}-2\langle k\rangle \mathcal{T}^{2}}
$$


However, in contrast to networks generated with stochastic rewiring algorithms, $\mathcal{A}$ can be attenuated either by increasing $\langle k\rangle$ or by setting the average number of triangles $\left\langle t_{\triangle}\right\rangle$ in order to obtain transitivity values close to $\mathcal{T}_{\text {max }}$, since $\mathcal{A}\left(\mathcal{T}_{\max }\right)=0$. Unfortunately, the strategy of increasing $\langle k\rangle$ comes with the price of decreasing $\mathcal{T}_{\text {max }}$. Nevertheless, even though $\mathcal{T}$ achieved for high $\langle k\rangle$ is not as high as the ones achieved by stochastic rewiring algorithms (88,98-101), it is possible to obtain a significantly higher clustering than those obtained in classical random network models $(60,83)$. There are, however, other network models $(60,95,96,102-105)$ that go beyond the low-clustering regime. For instance, an interesting generalization of the clustered random network $(60,83)$ was introduced in $(104)$, where networks can be constructed not only by single-edges and triangles, but also with arbitrary distributions of different kinds of subgraphs. In principle, one could mimic the subgraph structure of real-world networks using the model in (104). However, the implementation and analytical tractability of the model greatly increase as the connectivity pattern of the subgraphs becomes more complex. Another interesting model that can be suitably used to evaluate the dynamics of networks with similar topology as real structures was proposed in (105). Instead of focusing on how many triangles are attached to a given node, the model in $(95,96,105)$ is based on the concept of edge multiplicity, which is the number of triangles that a given edge participates. More specifically, each node is described by a $(N-1)$-dimensional vector $\boldsymbol{k}_{i}=\left(k_{i}^{(0)}, k_{i}^{(1)}, \ldots, k_{i}^{(M)}\right)$, where $k^{(l)}$ is the number of edges with multiplicity $l$ attached to node $i$ and $M=N-2$ is the maximum possible edge multiplicity. The total degree is then obtained by summing the contribution of all multiplicities, i.e. $k_{i}=\sum_{m=0}^{M} k_{i}^{(m)}$. The great advantage of the model based on edge multiplicities over other random models is that, while the evaluation of subgraph distributions in real networks is a potentially expensive task depending on the number of nodes, the distribution of edge multiplicities is easily calculated from real data, which can then be used as an input in the random model.(105) The analysis of the Kuramoto model and the development of MFAs in these and other random network models (106-116) for clustered networks are promising directions for future research. 


\section{Second-order Kuramoto Model on complex networks}

We have been pointing out the importance for an accurate modeling of topological properties found in real networks mentioning that one cannot expect to thoroughly comprehend dynamical aspects of these systems if their structure is not properly incorporated in the dynamical models. This need to go beyond mean-field theories for uncorrelated networks in the study of, for instance, epidemic or rumor spreading in social interactions is well motivated; as we discussed in the introduction of Chapter 3, it is blatantly apparent that the topology of social networks deviates significantly from traditional random network models $(18,60,104)$, hence investigations in the line of Chapter 3 in the context of social dynamics indeed have the potential to offer important insights in realistic scenarios. However, despite the many real-world applications that have been reported $(16,32)$ since its formulation, the connection of the Kuramoto model with real networked systems may not appear that obvious at first sight. This fact might also make the claims for a better understanding of real-world networks to seem futile if used to motivate the study of phase oscillators coupled in networks with more sophisticated topological patterns. Therefore, it is pertinent to pose the question: is there any real network whose dynamical behavior can be described by phase oscillators? It turns out that power-grids - systems that modern societies crucially depend on - can be modeled by inertial phase oscillators such as those described in Sec. 2.2.2.1. In fact, the crux for the stable and proper functioning of an electrical grid is the synchronous variation of its components' phases, a state which, if lost, can trigger massive blackouts such as the one that hit several European countries on November 2006 and that was caused by the obstruction of a single transmission line in Northwest Germany.(117) In view of these facts, it is vitally important to analyze how the emergence of collective dynamics in power systems is affected by the connectivity pattern, a problem that can be suitably tackled by employing the second-order Kuramoto model ${ }^{1}$.

Despite the progress achieved over the past two decades regarding synchronization phenomena on complex networks, the second-order Kuramoto model has attracted considerable less attention than its first-order version. In this chapter, we provide what turned out to be the first mean-field treatment of second-order Kuramoto oscillators in

1 There are, of course, classical and much more refined models in the electrical engineering literature that are designed to capture in detail the behavior of isolated units in an electrical grid. $(118,119)$ However, the simulation of many coupled units becomes a very expensive task due to the large number of parameters and variables in these models. On the other hand, although the Kuramoto model is not as accurate as the latter, it is simple enough to capture global aspects of the grid dynamics allowing, in this way, the simulation of networks with realistic sizes besides offering insights from nonlinear dynamics and statistical physics methods (see, for instance, $(26,120-122)$ ). 
uncorrelated networks. In particular, we focus on the case in which the natural frequencies are positively correlated with degrees (the reason for this will become clear shortly). Effects of degree-degree correlations - property observed in many real networks (18) are also investigated. Here, however, we do not address specific applications of the model to electric systems, but rather we concentrate ourselves on general aspects of the interplay between structure and dynamics. For reviews on phase oscillators studied in this context we refer to the reader to $(29,32)$ and references therein. This chapter is based on publications $(32,123-126)$, whose content will be closely followed in the next sections.

\subsection{Second-order Kuramoto model on uncorrelated networks}

\subsubsection{The model}

The second-order Kuramoto model consists of a population of $N$ coupled oscillators whose dynamics is governed by $(16,31,32,123)$ :

$$
\frac{d^{2} \theta_{i}}{d t^{2}}=-\alpha \frac{d \theta_{i}}{d t}+\omega_{i}+\lambda \sum_{j=1}^{N} A_{i j} \sin \left(\theta_{j}-\theta_{i}\right),
$$

In this chapter, we will assume that the natural frequencies are correlated with the degrees in the following form

$$
\omega_{i}=D\left(k_{i}-\langle k\rangle\right)
$$

where $D$ is a proportionality constant. This choice is a reminiscent of the work by GómezGardeñez et al. (127), where the authors for the first time tested the effects of such a correlation in the dynamics of first-order Kuramoto oscillators in networks. Seemingly naive, the choice $\omega_{i}=k_{i}$ adopted in (127) was shown to give rise to discontinuous synchronization phase transitions - or "explosive synchronization" as named by them -, which, until that moment, had remained unnoticed in the context of synchronization of phase oscillators on complex networks. Interestingly, the abrupt transition between asynchronous and synchronous state was reported to emerge only in networks with scale-free distributions. This finding motivated a myriad of works (see, for instance, (128-138)) investigating in more details the model introduced in (127) as well as the search for general dynamical rules that could induce abrupt transitions in any network topology. For a review on the explosive synchronization phenomenon we refer the reader to (32).

At first sight, the frequency assignment in Eq. 4.2 could seem odd or even unrealistic, raising perhaps the question about the relevance of studying this model. However, it is not difficult to find physical scenarios where this configuration is plausible. For example, this correlation between dynamics and network topology can arise as a consequence of a limited amount of resources or energy supply for the oscillators.(32,133,139) In fact, even 
before the work by Gómez-Gardeñez et al. came out, studies on optimization of synchronization in complex networks $(32,140-142)$ showed that, for a given fixed set of allowed frequencies $\left\{\omega_{1}, \omega_{2}, \ldots, \omega_{N}\right\}$, the frequency assignment configuration that maximizes the network synchronization is reached for cases in which frequencies are positively correlated with degrees. Therefore, this correlation between frequencies and local topology can be seen as an optimal scenario for the emergence of collective behavior in networks. Our goal in the next section is to combine the approaches of Sec. 2.2.1 and 2.2.2.1 in order to evaluate the collective dynamics of second-order Kuramoto oscillators in scale-free networks subjected to the frequency dependence in Eq. 4.2.

\subsubsection{Mean-field theory}

Substituting Eq. 4.2 into 4.1 and using the same ideas in Sec. 2.2.1, we can formulate the previous equation in the continuum limit as

$$
\frac{d^{2} \theta}{d t^{2}}=-\alpha \frac{d \theta}{d t}+D(k-\langle k\rangle)+\frac{\lambda k}{\langle k\rangle} \iint k^{\prime} P\left(k^{\prime}\right) \rho\left(\theta^{\prime}, t \mid k^{\prime}\right) \sin \left(\theta^{\prime}-\theta\right) d \theta^{\prime} d k^{\prime}
$$

which rewritten in terms of the mean-field quantities becomes

$$
\ddot{\theta}=-\alpha \dot{\theta}+D(k-\langle k\rangle)+k \lambda r \sin (\psi-\theta),
$$

where the order parameter $r$ is defined by

$$
r e^{i \psi}=\frac{1}{\langle k\rangle} \iint k P(k) \rho(\theta, t \mid k) e^{i \theta} d \theta d k .
$$

Setting the reference frame $\phi(t)=\theta(t)-\psi(t)$ and defining $C(\lambda r) \equiv(\ddot{\psi}+\alpha \dot{\psi}) / D$ we obtain

$$
\ddot{\phi}=-\alpha \dot{\phi}+D[k-\langle k\rangle-C(\lambda r)]-k \lambda r \sin \phi .
$$

As in the previous sections, our initial goal is to calculate how the global synchronization of the population of oscillators in Eq. 4.1 is modified by changes in the coupling strength $\lambda$. To this end, let us assume that all locked oscillators have degree $k$ in a certain range $k \in k_{\text {lock }} \equiv\left[k_{1}, k_{2}\right]$. These oscillators evolve coherently and are thus characterized by $\dot{\phi}=\ddot{\phi}=0$, defining in this way the stable fixed point solution $\phi=\arcsin \left(\frac{D(k-\langle k\rangle-C(\lambda r))}{k \lambda r}\right)$, which is a $k$-dependent constant phase. Correspondingly, the time-independent phase density $\rho_{\text {lock }}(\phi \mid k)$ of locked oscillators with phase $\phi$ for a given degree $k$ is given by

$$
\rho_{\text {lock }}(\phi \mid k)=\delta\left[\phi-\arcsin \left(\frac{D(k-\langle k\rangle-C(\lambda r))}{k \lambda r}\right)\right] \text { for } k \in\left[k_{1}, k_{2}\right],
$$

where $\delta$ is the Dirac delta function. The contribution of locked oscillators is then expressed as

$$
r_{\text {lock }}=\frac{1}{\langle k\rangle} \int_{k_{1}}^{k_{2}} \int_{0}^{2 \pi} P(k) k e^{i \phi(t)} \delta\left[\phi-\arcsin \left(\frac{D(k-\langle k\rangle-C(\lambda r))}{k \lambda r}\right)\right] d \phi d k,
$$


whose real part yields

$$
r_{\text {lock }}=\frac{1}{\langle k\rangle} \int_{k_{1}}^{k_{2}} k P(k) \sqrt{1-\left[\frac{D(k-\langle k\rangle-C(\lambda r))}{k \lambda r}\right]^{2}} d k .
$$

Let us consider a SF degree distribution given by $P(k)=A(\gamma) k^{-\gamma}$, where $A(\gamma)$ is the normalization factor and $\gamma=3$. Substituting the degree distribution $P(k)$ and defining $x(k)=D(k-\langle k\rangle-C(\lambda r)) / \lambda k r$, we obtain the following implicit equation for the contribution of the locked oscillators

$$
\begin{aligned}
r_{\text {lock }} & =\frac{A(\gamma)}{2 D\langle k\rangle}\left[\left(x\left(k_{2}\right) \sqrt{1-x^{2}\left(k_{2}\right)}\right)+\arcsin x\left(k_{2}\right)\right. \\
& \left.-\left(x\left(k_{1}\right) \sqrt{1-x^{2}\left(k_{1}\right)}+\arcsin x\left(k_{2}\right)\right)\right] .
\end{aligned}
$$

Having defined that the locked oscillators are the nodes with degree within the range $k \in\left[k_{1}, k_{2}\right]$, the drifting oscillators are then given by the nodes with degree $k \in k_{\text {drift }} \equiv\left[k_{\min }, k_{1}\right] \cup\left[k_{2}, k_{\max }\right]$, where $k_{\min }$ denotes the minimal degree and $k_{\max }$ is the maximal degree. To determine the contribution of these oscillators to the total order parameter we need to employ the same procedure as exposed in Sec. 2.2.2.1, since the presence of the inertial term stops us from deriving directly the phase density $\rho_{\text {drift }}(\phi \mid k)$ as in the first-order model. Analogously to Eq. 2.43, in the stationary state we have that density of oscillators with phase $\phi$ for a given degree $k$ follows $\rho_{\text {drift }}(\phi \mid k) \sim|\dot{\phi}(k)|^{-1}$. Similarly as before, given that $\rho_{\text {drift }}$ is a $2 \pi$-periodic function and the normalization condition $\int_{0}^{2 \pi} \rho_{\text {drift }}(\phi \mid k) d \phi=\int_{0}^{\tilde{T}} \rho_{\text {drift }}|\dot{\phi}|^{-1} d t$, we obtain

$$
\rho_{\text {drift }}(\phi \mid k)=\tilde{T}|\dot{\phi}|^{-1}=\frac{\tilde{\omega}}{2 \pi}|\dot{\phi}|^{-1},
$$

where $\tilde{T}$ and $\tilde{\omega}$ are the period and the frequency of the running periodic solution of $\phi .(31)$ Inserting Eq. 4.9 into Eq. 4.4, we get

$$
r_{\mathrm{drift}}=\frac{1}{2 \pi\langle k\rangle} \int_{k \in k_{\mathrm{drift}}} \int_{0}^{\tilde{T}} k P(k) \tilde{\omega}|\dot{\phi}|^{-1} \dot{\phi} e^{i \phi(t)} d t d k .
$$

Since $\dot{\phi}<0$ for $k \in\left[k_{\min }, k_{1}\right]$ and $\dot{\phi}>0$ for $k \in\left[k_{2}, k_{\mathrm{max}}\right]$, the real part of equation (4.10) becomes

$$
r_{\text {drift }}=\frac{1}{\pi\langle k\rangle}\left(-\int_{k_{\min }}^{k_{1}}+\int_{k_{2}}^{k_{\max }}\right) \int_{0}^{\tilde{T}} k P(k) \tilde{\omega} \cos \phi(t, k) d t d k .
$$

Using the approximation for $\int_{0}^{\tilde{T}} \cos \phi(t, k) d t$ in Appendix A and performing some manipulations motivated by (31), we obtain

$$
r_{\mathrm{drift}}=\left(-\int_{k_{\min }}^{k_{1}}+\int_{k_{2}}^{k_{\max }}\right) \frac{-r k^{2} \lambda \alpha^{4} P(k)}{D^{3}[k-\langle k\rangle-C(\lambda r)]^{2}\langle k\rangle} d k
$$

Thus, the self-consistent equation for $r$ is obtained by summing the contribution of locked and drifting oscillator as

$$
r=r_{\text {lock }}+r_{\text {drift }}
$$


which are obtained from Eqs. (4.7) and (4.12), respectively.

If the degree distribution were symmetric, the analysis to determine the dependence of the order parameter $r$ on the coupling strength $\lambda$ would end here. Yet, the natural frequencies of our problem are positively correlated with the degrees (Eq. 4.2), leading, in the case treated here, to an asymmetric frequency distribution due to our choice of having SF networks as the substrate for the dynamics. This fact makes the imaginary parts of Eqs. 4.6 and 4.10 to be nonzero, in contrast with the situation presented in Sec. 2.2.2. Furthermore, there is still a crucial quantity to be determined - apart, of course, from the ranges $k_{\text {lock }}$ and $k_{\text {drift }}$, which we will soon address -, namely the constant $C(\lambda r)$. Let us then consider again the sum of Eqs. (4.6) and (4.10). Taking its imaginary part, we get

$$
\begin{aligned}
0 & =\frac{1}{\langle k\rangle} \int_{k_{1}}^{k_{2}} k P(k) \frac{D[k-\langle k\rangle-C(\lambda r)]}{k \lambda r} d k \\
& +\frac{1}{2 \pi\langle k\rangle}\left(-\int_{k_{\min }}^{k_{1}}+\int_{k_{2}}^{k_{\max }}\right) \int_{0}^{T} k P(k) \tilde{\omega} \sin \phi(t, k) d t d k .
\end{aligned}
$$

The solution of the previous equation requires the estimation of the integral $\int_{0}^{\tilde{T}} \sin \phi(t) d t$, whose calculation closely follows the procedure for $\int_{0}^{\tilde{T}} \cos \phi(t) d t(31)$ and it is presented in details in Appendix A. Using this result, we obtain

$$
\begin{aligned}
0= & \frac{1}{\langle k\rangle} \int_{k_{1}}^{k_{2}} k P(k) \frac{D[k-\langle k\rangle-C(\lambda r)]}{k \lambda r} d k \\
& +\frac{1}{2\langle k\rangle}\left(-\int_{k_{\min }}^{k_{1}}+\int_{k_{2}}^{k_{\max }}\right) \frac{r k^{2} \lambda \alpha^{2} P(k)}{D^{2}[k-\langle k\rangle-C(\lambda r)]^{3}} d k
\end{aligned}
$$

Finally, we have the full recipe to calculate parameter $r$. Precisely, through Eq. (4.15) we yield the solution of $C(\lambda r)$ as a function of the coupling $\lambda$. This result is then inserted in the self-consistent equation generated by the sum of Eqs. 4.7 and 4.12 from which the dependence $r=r(\lambda)$ can be assessed. Now we came to the final puzzle: which values for the range of degrees should we use in Eqs. 4.7, 4.12 and 4.15? And how do we distinguish them in the experiments where we increase and decrease $\lambda$ ? This is the subject of the next section.

\subsubsection{Synchronization boundaries}

The determination of the range of degrees when the coupling is progressively increased and decreased can be illustrated using the parameter space of damped driven pendulumn discussed in Sec. 2.2.2.1. Let us for convenience nondimensionalize Eq. 4.5 by setting the new time-scale $\tau=t \sqrt{k \lambda r}$ :

$$
\frac{d^{2} \phi}{d \tau^{2}}+\beta \frac{d \phi}{d \tau}+\sin \phi=I
$$


where $\beta=\alpha / \sqrt{\lambda k r}$ and $I=D[k-\langle k\rangle-C(\lambda r)] / k \lambda r$ are the dimensionless damping and equivalent torque applied, respectively. The bifurcation diagram of Eq. 4.16 can be summarized by the existence of a stable fixed point and a stable limit cycle solutions which are mediated by saddle-node, homoclinic and infinite-period bifurcations in the $\beta-I$ plane (see Fig. 3). Recalling, in terms of the collective dynamics the parameter space of Fig. 3 can be translated as follows: starting the dynamics with sufficiently small couplings initially places the oscillators in the stable limit cycle region $(I>1)$, given that $I \propto 1 / \lambda$. In this region, only periodic solutions in the phase space $\phi-\dot{\phi}$ are allowed and, since each one of these solutions depends on $k$, nodes end up converging to different limit cycles and, consequently, oscillating at different rhythms. As the coupling is increased, nodes start entering in the region of bistability, but remain oscillating incoherently until the homoclinic bifurcation line is crossed $(I=4 \beta / \pi)$, where the stable limit cycle is destroyed and the trajectory $\phi(t)$ converges to the stable fixed point $\phi=\arcsin [D(k-\langle k\rangle-C(\lambda r)) / \lambda k r]$. The larger the coupling, the higher the number of oscillators that are attracted to their respective fixed points, expanding, in this way, the size of the synchronous component. Figure 9(a) illustrates this process by showing the trajectory in the parameter space $\beta-I$ of two randomly selected oscillators with degrees $k=8$ and 20. At low coupling values, both nodes are in the stable limit cycle region; however, with the increasing of $\lambda$, the oscillator with $k=8$ joins first the stable fixed point area, while larger couplings are required to attract highly connected nodes. Suppose now that the completely synchronous state is achieved - i.e. the whole population is in the region of $I<4 \beta / \pi$ in Fig. 3. Starting with this configuration, the oscillators will only be brought back to incoherence when $I=1$, point at which a saddle-node bifurcaton occurs. In this case, the dismantling of the synchronous component is initiated by the higher degree nodes (see Fig. 9 (b)).

After the analysis above we conclude that if the system is initiated in a stable limit cycle area, the stable fixed point is only reached if the conditions $I \leq 1$ and $I \leq 4 \beta / \pi$ are satisfied. This leads to the following inequalities

$$
\frac{|D[k-\langle k\rangle-C(\lambda r)]|}{k \lambda r} \leq 1 \text { and } \frac{|D[k-\langle k\rangle-C(\lambda r)]|}{k \lambda r} \leq \frac{4 \alpha}{\pi \sqrt{k \lambda r}} .
$$

From Eq. 4.17 we derive that the range of degrees of the synchronous nodes for the case in which the coupling is increased is given by

$$
k^{\mathrm{I}} \in\left[k_{1}^{\mathrm{I}}, k_{2}^{\mathrm{I}}\right]=\left[\frac{B-\sqrt{B^{2}-4 D^{4}[\langle k\rangle+C(\lambda r)]^{2}}}{2 D^{2}}, \frac{B+\sqrt{B^{2}-4 D^{4}[\langle k\rangle+C(\lambda r)]^{2}}}{2 D^{2}}\right],
$$

where

$$
B=2 D^{2}[\langle k\rangle+C(\lambda r)]+\frac{16 \alpha^{2} \lambda r}{\pi^{2}} .
$$

When the coupling is decreased from a sufficient high value $\lambda_{0}$ that ensures that the completely synchronous state was achieved, all oscillators remain locked until $I<1$ is 

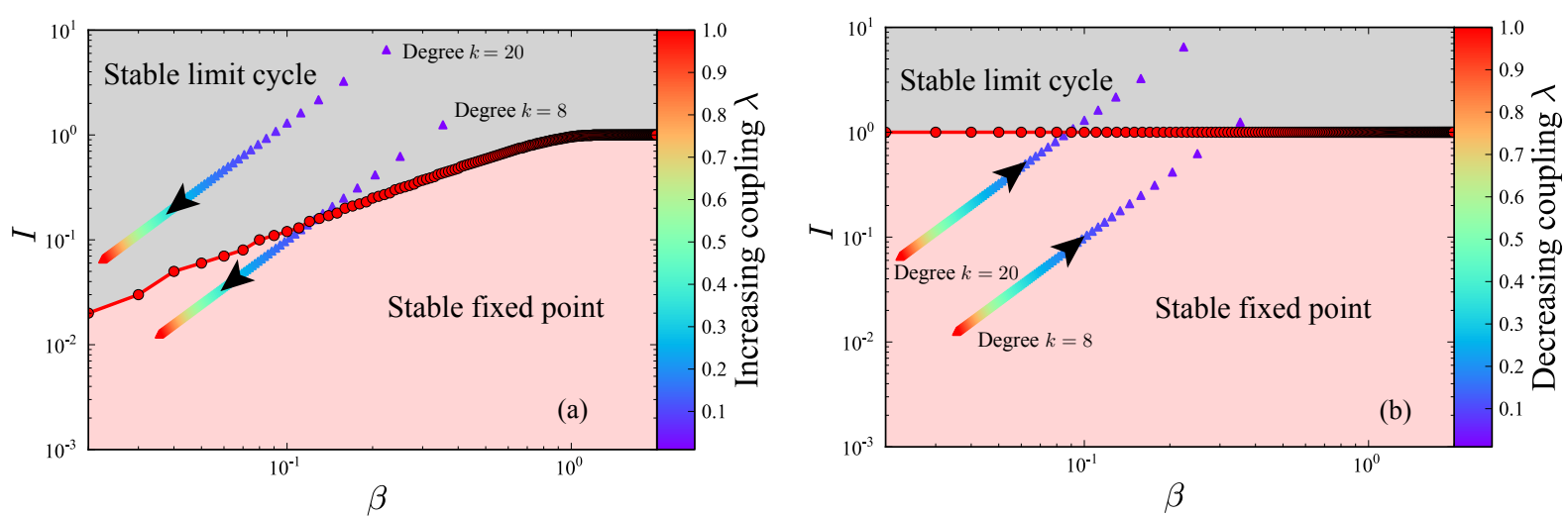

Figure 9 - Parameter space of Eq. 4.16 for when the coupling is (a) increased and (b) decreased. The red area represents the region with the stable fixed point solution, whereas the gray area indicates the parameter combinations that yield stable limit cycles. The triangles show the evolution in the parameter space of oscillators with degree $k=8$ and 20, starting in incoherence in (a) [coherence in (b)] and approach the synchronous state (incoherence) as the coupling is increased (decreased). Parameters: $\alpha=0.1$, $D=0.1,\langle k\rangle=10, N=3000$, and $P(k) \propto k^{-\gamma}$, with $\gamma=3$.

Source: Adapted from JI et al. (124)

satisfied. The latter inequality yields the range of synchronous degrees

$$
k^{\mathrm{D}} \in\left[k_{1}^{\mathrm{D}}, k_{2}^{\mathrm{D}}\right]=\left[\frac{\langle k\rangle+C(\lambda r)}{1+\frac{\lambda r}{D}}, \frac{\langle k\rangle+C(\lambda r)}{1-\frac{\lambda r}{D}}\right]
$$

Note that the determination of the $r(\lambda)$ for increasing and decreasing coupling strategies depends entirely on the ranges $k^{\mathrm{I}}$ and $k^{\mathrm{D}}$; the expressions for $r$ (Eqs. 4.7, 4.12 and 4.15) in both cases are the same, only the integration limits differ one case from the other.

\subsubsection{Mean-field theory vs. numerical experiments}

In this section we compare the results obtained via the mean-field calculations of the previous section with numerical experiments. All simulations performed here consist in evolving Eqs. 4.1 with $\alpha=0.1$ and $D=0.1$ considering SF networks as the underlying topology with $N=3000,\langle k\rangle=10, k_{\min }=5$ and degree distribution $P(k) \sim k^{-\gamma}$, where $\gamma=3$ (BA model). Since we expect the system to exhibit some hysteretic behavior, the simulations are carried out as follows: firstly, after the system is initialized with random initial conditions for $\left\{\theta_{i}\right\}$ and $\left\{\dot{\theta}_{i}\right\}$, the coupling strength $\lambda$ is increased from a given $\lambda_{0}$ by amounts $\delta \lambda=0.1$ and the order parameter $r^{\mathrm{I}}$ is computed for $\lambda=\lambda_{0}, \lambda_{0}+\delta \lambda, \cdots, \lambda_{0}+n \delta \lambda$. Subsequently, $\lambda$ is gradually decreased from $\lambda_{0}+n \delta \lambda$ again in steps $\delta \lambda$. At each coupling step, the system is integrated long enough ( $10^{5}$ time steps) using a fourth-order RungeKutta method with time step $d t=0.01$. 

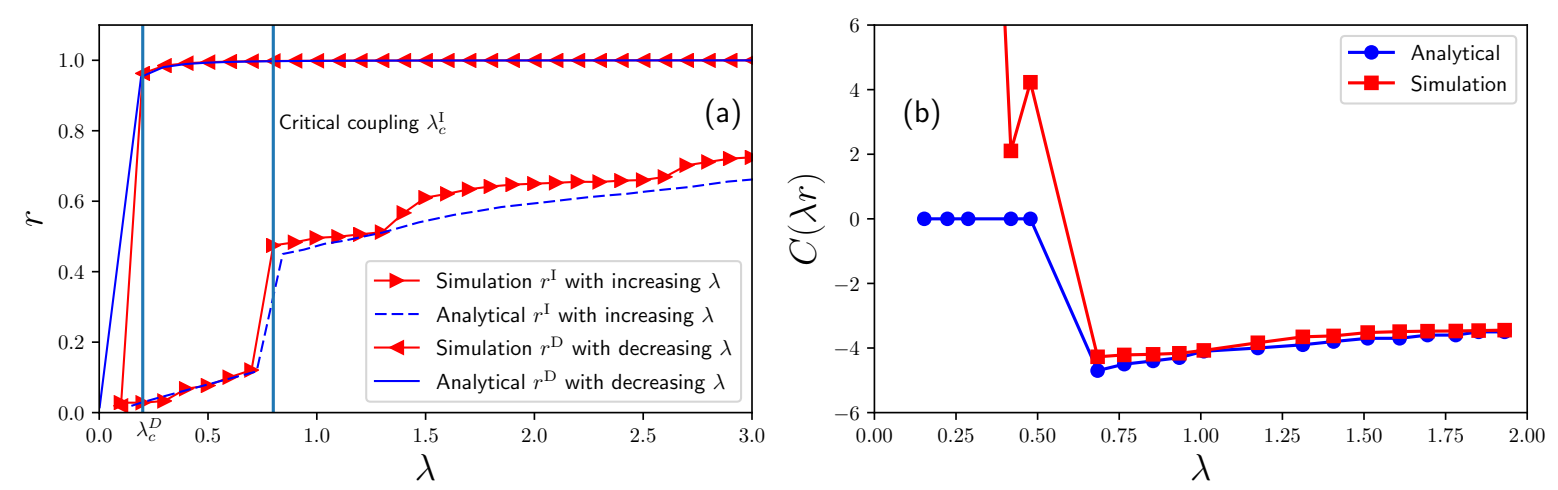

Figure 10 - (a) Analytical and numerical curves of the order parameter $r$ and (b) evolution of $C(\lambda r)$ for the case in which the coupling strength is increased. For the analytical curve, we set $C(\lambda r)=0$ for $\lambda<\lambda_{c}^{\mathrm{I}}$. In both panels the theoretical results are derived from Eqs. 4.8, 4.13, 4.15 and 4.18 using the range of synchronous degrees for increasing (Eq. 4.18) and decreasing (Eq. 4.20) coupling strength. Parameters of the model: $\alpha=0.1, D=0.1$. Simulation parameters: SF network with $N=3000$ nodes, $\langle k\rangle=10, k_{\min }=5$ and degree distribution $P(k) \sim k^{-\gamma}$, with $\gamma=3$.

Source: Adapted from JI et al. (124)
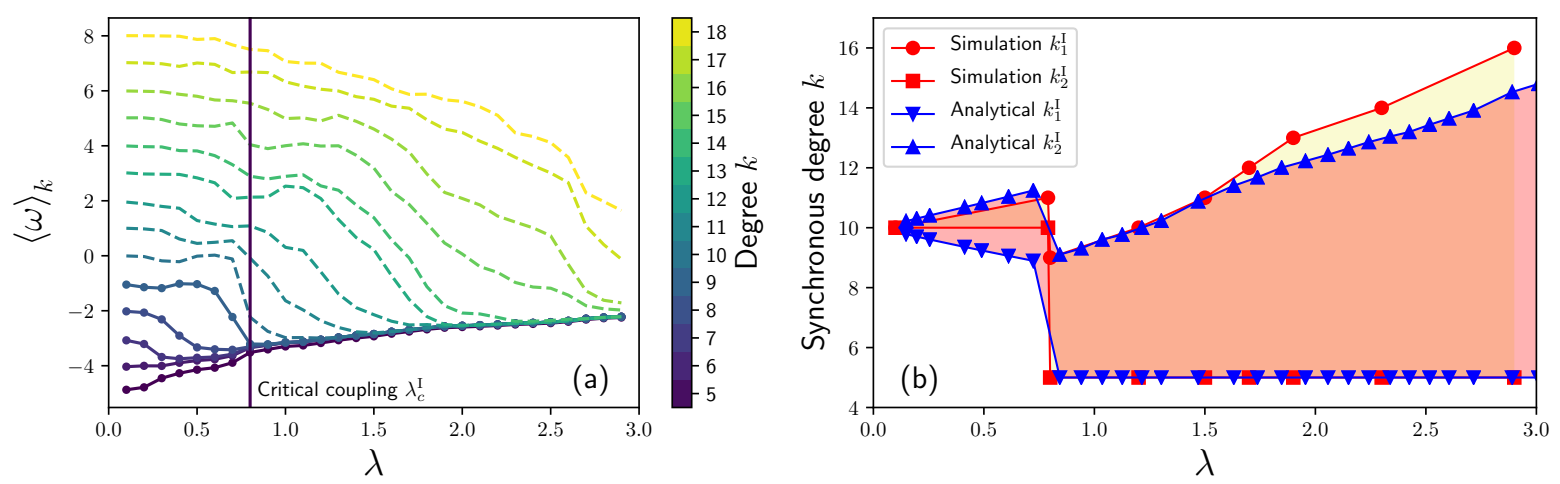

Figure 11 - (a) Average frequency $\langle\omega\rangle_{k}$ of oscillators with same degree $k$ and (b) range of degrees of synchronous nodes. The simulation parameters are the same as in Fig. 10.

Source: Adapted from JI et al. (124)

Figure 10(a) shows the synchronization curves of system (4.1) considering the parameters above. As expected, the system indeed shows hysteretic synchrony. When the coupling is increased, oscillators that are initially drifting are gradually entrained by the mean-field, causing the order parameter $r$ to slowly increase until $\lambda_{c}^{\mathrm{I}}$, value at which an abrupt synchronization transition occurs. After $\lambda_{c}^{\mathrm{I}}$, the network synchronous component grows steadily, as shown by the continuous increase of $r$ for $\lambda>\lambda_{c}^{\mathrm{I}}$. For high coupling strengths, the population is placed in the completely synchronous state $(r=1)$, which is extinguished at $\lambda=\lambda_{c}^{\mathrm{D}}$ [see Fig. 10(a)]. In order to validate the mean-field calculations with the simulation results, we simultaneously solve Eq. 4.18 (4.20) for the increasing 

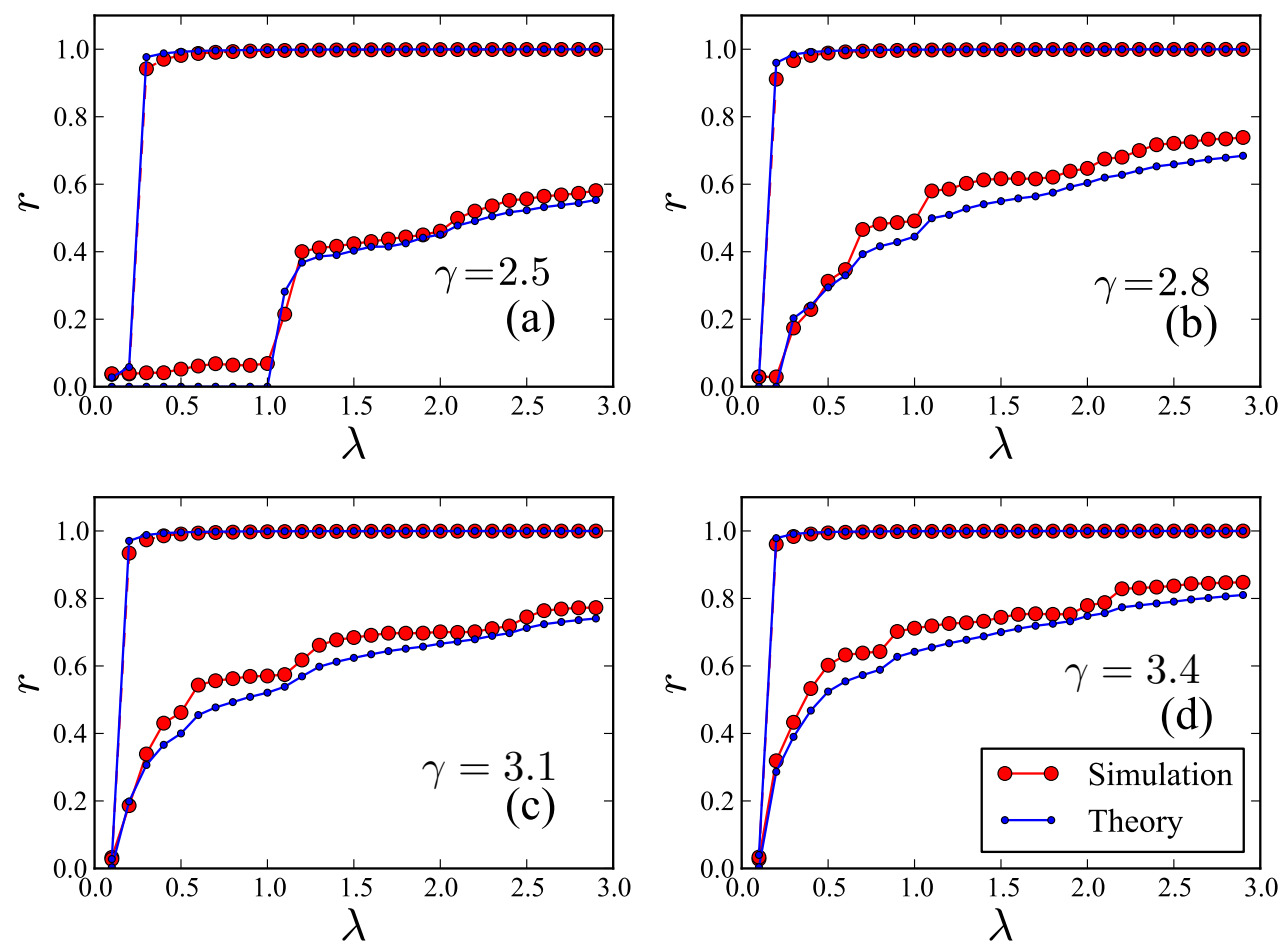

Figure 12 - Synchronization diagrams for networks with degree distribution $P(k) \sim k^{-\gamma}$ for different exponent $\gamma$. Lower (upper) curves correspond to the forward (backward) propagation of the coupling strength. The analytical plots are calculated from the summation of Eqs. 4.22 and 4.24. All networks have $N=3000$ and $\langle k\rangle=10$.

Source: Adapted from JI et al. (124)

(decreasing) coupling cases. As it can be seen in Fig. 10(a), a good agreement is obtained between the analytical and numerical results. One should note that the distribution of natural frequencies is proportional to the degrees, hence $\psi$ cannot be set to a constant. Instead the terms $\dot{\psi}$ and $\ddot{\psi}$ contribute to $C(\lambda r)$, whose solution is obtained through Eq. 4.15. The evolution of $C(\lambda r)$ as a function of $\lambda$ is depicted in Fig. 10(b).

It is interesting to point out some qualitative differences between the synchronization diagrams of SF and fully connected networks. Specifically, in Fig. 10 we see that after the abrupt transtion at $\lambda_{c}^{\mathrm{I}}$ we have a progressive formation of the synchronous component. On the other hand, the population of globally coupled oscillators in Fig. 4 is already very close to the fully synchronized for $\lambda>\lambda_{c}^{\mathrm{I}}$. Differences in the behavior of $r$ can also be noticed for $\lambda<\lambda_{c}^{\mathrm{I}}$. In particular, as predicted by the mean-field calculations in Sec. 2.2.2, in Fig. 4 no noticeable synchronization $(r \approx 0)$ is measured in for $\lambda<\lambda_{c}^{\mathrm{I}}$, whereas the oscillators in SF networks indeed exihibit partial synchronization in the same region. Seeking a better understanding of this phenomenon, in Fig. 11 we show the evolution of the average frequency of groups of oscillator with the same degree $k$, i.e. $\langle\omega\rangle_{k}=\sum_{i \mid k_{i}=k} \omega_{i}^{\text {eff }} / N_{k}$, where $\omega_{i}^{\text {eff }}=\int_{t}^{t+T} \dot{\theta}_{i}(\tau) d \tau / T$ with $t$ being sufficiently large in order to overcome transient effects. Comparing Figs. 10 and 11(a), we observe that the non-vanishing synchronization values 
for $\lambda<\lambda_{c}^{\mathrm{I}}$ are due to the formation of a synchronous cluster containing nodes with low degrees. As the coupling is further increased, nodes keep joining this cluster successively according to their degrees even after the occurrence of the abrupt transition at $\lambda_{c}^{\mathrm{I}}$. The range of degrees corresponding to oscillators entrained by the mean-field is depicted in detail in Fig. 11(b). Not only is the behavior observed in Fig. 11(a) in contrast with the dynamics in the fully connected graph, but it also differs from the discontinuity transition found in SF networks of first-order Kuramoto oscillators under correlation between frequencies and degrees reported in (127), where it is shown that all nodes abruptly merge into a giant synchronous component at $\lambda_{c}^{\mathrm{I}}$. In other words, in the absence of inertia, at the critical coupling the whole network is brought to the vicinity of a perfectly synchronized state, while in populations of second-order oscillators the number of locked nodes is progressively expanded. In order to highlight the differences between the findings in (127) for first-order Kuramoto model and the cascades of transitions towards the synchronous state by nodes grouped in clusters of nodes with the same degree observed in Figs. 10 and 11, we refer to the latter phenomenon as cluster explosive synchronization $(123,124)$.

We proceed with discussing the dynamical behavior of inertial phase oscillators in networks with different levels of structural heterogeneity. In Fig. 12 is shown the synchronization diagrams for the forward and backward continuation of the coupling strength considering SF networks with different exponents $\gamma$. As expected, the onset of synchronization is decreased in the forward branch for higher $\gamma$, since the more heavily right-skewed the power-law degree distribution, the greater the difference between the highest degree oscillators with those with degrees close to the average, requiring then higher couplings to lock these oscillators in synchrony. Noteworthy, the branch associated to the backward propagation of $\lambda$ is barely affected by changes in $\gamma$. A similar effect was recently reported in (143) with respect to variations of the system size $N$.

\subsubsection{Disturbing the correlation between frequencies and degrees}

In the previous subsections we saw the effects of correlating frequencies and degrees on the collective dynamics of second-order Kuramoto oscillators in SF networks. It is natural to ask then how these results change if this relation between intrinsic dynamics and local topology is now disturbed. One of the motivations for this analysis can be found in the evidences discussed in Sec. 4.1.1. One may recall from that section that the emergence of dependencies of natural frequencies on degrees is related with the optimization of the network synchrony given certain topological constraints.(32, 140-142) Therefore, disturbances to the perfect correlation imposed by Eq. 4.2 could potentially model local maxima in synchronization optimization processes that work via changes in the frequency assignment. To address this issue, we consider the inclusion of quenched disorder terms in the natural frequencies as $\Omega_{i}=D\left(k_{i}-\langle k\rangle\right)+\varepsilon_{i}$, where $\varepsilon_{i} \in[-q, q]$ is randomly draw 

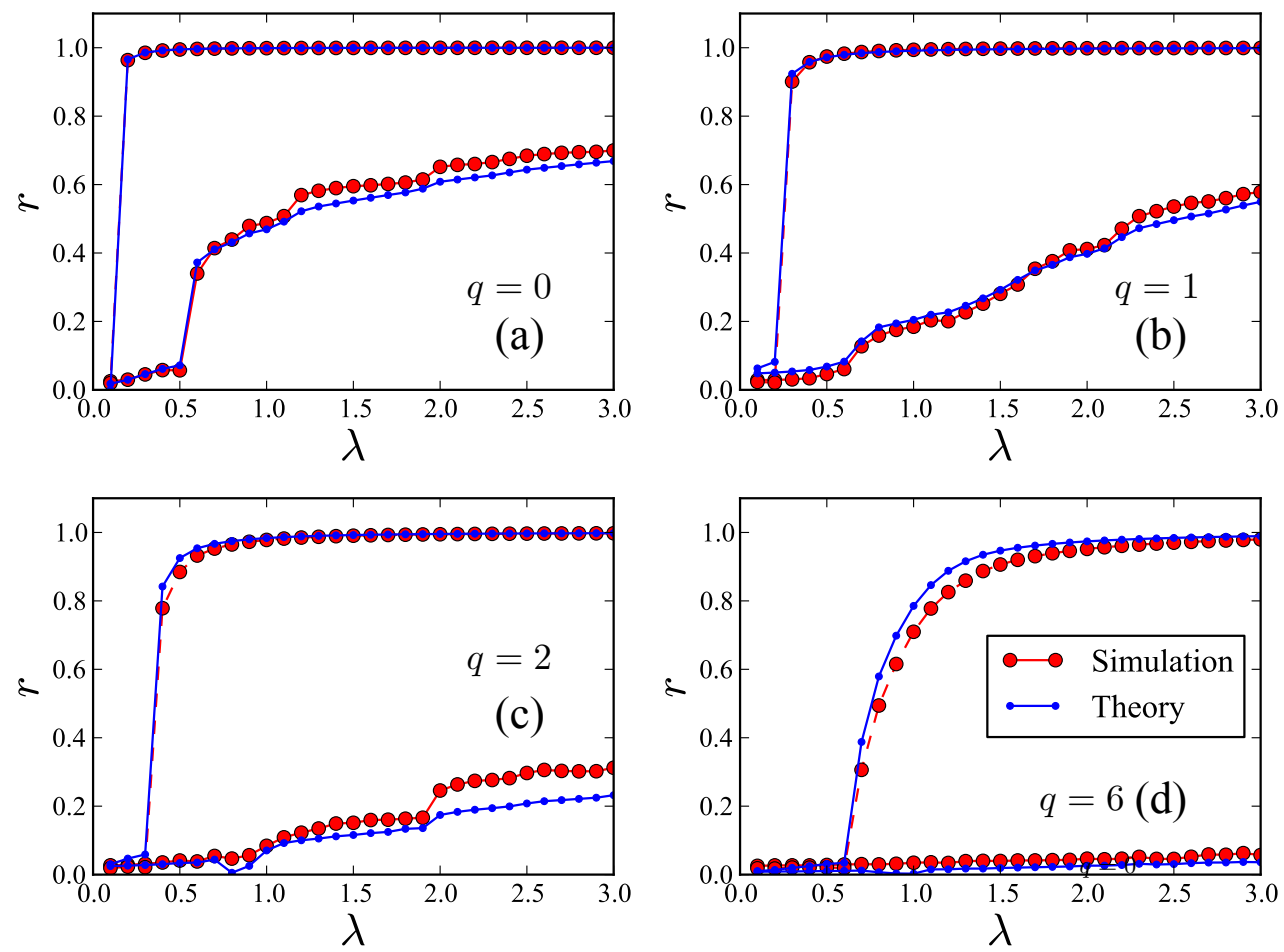

Figure 13 - Synchronization diagrams for different values of the disorder parameter $q$. Analytical results are obtained from the summation of $r_{\text {lock }}^{\mathrm{q}}$ and $r_{\mathrm{drift}}^{\mathrm{q}}$. The same network topology used in Fig. 2 was used in the simulations of all panels in this figure. Lower (upper) curves correspond to the forward (backward) propagation of the coupling strength. Other parameters: $N=3000,\langle k\rangle=10, \alpha=0.1, D=0.1$ and distribution of quenched disorder as $g(\varepsilon)=1 / 2 \varepsilon$ for $\varepsilon \in[-q, q]$.

Source: Adapted from JI et al. (124)

from a uniform distribution $g(\varepsilon)$. Upon substitution in Eq. 4.1 and its formulation in the continuum limit we have

$$
\ddot{\phi}=-\alpha \dot{\phi}+D[k-\langle k\rangle-C(\lambda r)]+\varepsilon-k \lambda r \sin \phi .
$$

Thus, as the magnitude $q$ increases, the topological influence on the natural frequencies is dominated by the quenched disorder terms $\varepsilon$. The contribution of locked oscillators $r_{\text {lock }}^{\mathrm{q}}$ is calculated through

$$
r_{\text {lock }}^{\mathrm{q}}=\frac{1}{\langle k\rangle} \int_{-q}^{q} \int_{k_{1}^{\mathrm{q}}(\varepsilon)}^{k_{2}^{\mathrm{q}}(\varepsilon)} k P(k) g(\varepsilon) \sqrt{1-\left[\frac{D(k-\langle k\rangle-C(\lambda r))+\varepsilon}{k \lambda r}\right]^{2}} d k d \varepsilon
$$

where the degree range of the synchronous oscillators $k^{\mathrm{q}} \in\left[k_{1}^{\mathrm{q}}(\varepsilon), k_{2}^{\mathrm{q}}(\varepsilon)\right]$ for the increasing coupling case is determined by the conditions

$$
\frac{|D[k-\langle k\rangle-C(\lambda r)]+\varepsilon|}{k \lambda r} \leq 1 \text { and } \frac{|D[k-\langle k\rangle-C(\lambda r)]+\varepsilon|}{k \lambda r} \leq \frac{4 \alpha}{\sqrt{k \lambda r}}
$$


When the coupling is decreased, the range of synchronous degrees is obtained only via the first inequality in the equation above. Analogously to Eq. 4.10, the contribution of the drifting oscillators $r_{\mathrm{drift}}^{\mathrm{q}}$ is estimated by

$$
\begin{aligned}
r_{\mathrm{drift}}^{\mathrm{q}}= & -\int d \varepsilon\left(\int_{k_{\min }}^{k_{1}^{\mathrm{q}}(\varepsilon)} d k+\int_{k_{2}^{\mathrm{q}}(\varepsilon)}^{k_{\max }} d k\right) \\
& \times \frac{-r k^{2} \lambda \alpha^{4} P(k) g(\varepsilon)}{[D(k-\langle k\rangle-C(\lambda r))+\varepsilon]^{2}\langle k\rangle} \Theta(D[\langle k\rangle+C(\lambda r)-k]-\varepsilon) \\
+ & \int d \varepsilon\left(\int_{k_{\min }}^{k_{1}^{\mathrm{q}}(\varepsilon)} d k+\int_{k_{2}^{\mathrm{q}}(\varepsilon)}^{k_{\max }} d k\right) \\
\times & \frac{-r k^{2} \lambda \alpha^{4} P(k) g(\varepsilon)}{[D(k-\langle k\rangle-C(\lambda r))+\varepsilon]^{2}\langle k\rangle} \Theta(D[k-\langle k\rangle-C(\lambda r)]+\varepsilon),
\end{aligned}
$$

where $\Theta(\cdot)$ is the Heaviside function. In Fig. 13 we show the synchronization diagrams for the same network topology used to obtain the results in Fig. 10, but considering oscillators whose natural frequencies are perturbed by quenched disorder terms as in Eq. 4.21. The analytical curves for the order parameter are calculated exactly as before; the process to solve the equations for $r_{\text {lock }}^{\mathrm{q}}$ and $r_{\text {drift }}^{\mathrm{q}}$ only differs in the integration limits associated to variable $k$. As it is seen in Fig. 13, the onset of synchronization associated to the lower curves tends to increase with $q$, a fact already expected since the higher the width of the frequency distribution, the higher the couplings needed to synchronize the highly heterogeneous oscillators. At last, an interesting phenomenon occurs when the perturbation in the frequencies are included, namely the abrupticity of both synchronization transitions is significantly reduced. While the upper branch of $r$ is more strongly affected only at the high values of $q$ [Fig. 13(d)], the transition towards the synchronous state for increasing coupling case already looses its apparent discontinuity for $q=1$ [Fig. 13(b)]. Of particular interest is the contrast of the smoothing process of the backward transition in Fig. 13(d) with the results on the first-order Kuramoto model reported in (144). There, it was found that the inclusion of quenched disorder in fact promotes the occurrence of discontinuous transitions as well as the hysteretic behavior of the order parameter.

\subsection{Second-order Kuramoto model in correlated networks}

We started this chapter motivated by the importance of the second-order $\mathrm{Ku}$ ramoto model in the description of a real-world complex system that is crucial for the functioning of modern society (power-grids). Bearing in mind this application perspective, we, therefore, could not conclude the study developed thus far without investigating the influence of properties that are found not only in power-grids, but are also pervasive in 
many real networks.(18) In principle, one could carry a similar analysis as the one in Chapter 3; that is, adapt the heterogeneous degree mean-field theory to the Newman-Miller model of singles-edges and triangles and then evaluate how clustering affects the global dynamics of inertial oscillators. Although, on the one hand, this approach seems relevant for the study of real networks modeled by Kuramoto oscillators (power-grids often exhibit non-vanishing values for the clustering coefficient $(71,145,146))$, on the other hand, the low-clustering limit of the model in $(60,83)$ makes us suspicious about observing once again the innocuous effect of triadic connections showed in Sec. 3.4 and reported in many other papers $(32,71,93,94)$. Indeed, as numerically verified (simulations not shown here), all conclusions of Chapter 3 also hold for the second-order model, i.e. tree-based theories accurately describe the dynamics of networks with moderate values of clustering even if the inertia related to the phases is not negligible. To what extent the number of triangles can be increased until the mean-field calculations start to fail and how this depends on the oscillator model are intricate questions that we leave for future works. In this section, nevertheless, we consider the effects of degree-degree correlations on the oscillators' collective behavior by considering networks generated with the stochastic rewiring algorithm proposed in (90). The advantage of (90) over considering again the Newman-Miller model is that the former yields networks with tunable assortativity and with clustering levels beyond the low-clustering regime; however, as all stochastic rewiring methods, its application comes with the unfortunate drawback of lacking analytical tractability, a price that one has to pay if the comprehension of extreme topological characteristics is sought.

The method to tune the network's assortativity works as follows: at each step of the algorithm, two edges are selected at random and the four nodes associated to these edges are ordered from the lowest to the highest degree. In order to produce assortative mixing $(\mathcal{A}>0)$, with a probability $p$, a new edge connects the first and the second node and another new edge links the third and fourth nodes. If one of the two new edges already exists, the step is discarded and a new pair of edges is chosen. This same heuristic can also generate dissortative networks $(\mathcal{A}<0)$ with only a slight change in the algorithm. After selecting the four nodes and sorting them with respect to their degrees, one must rewire, with probability $p$, the highest degree node with the lowest one and, likewise, the second and third nodes. After rewiring the network, if the degree of assortativity or dissortativity is higher or smaller than the desired $\mathcal{A}, p$ is decreased or increased respectively and the network is rewired following the procedures described above.

All the simulations performed in this section follow the same protocol as previously, that is, the coupling strength is adiabatically increased by amounts $\delta \lambda$ from an initial value $\lambda_{0}$ and for each $\lambda_{0}, \lambda_{0}+\delta \lambda, \ldots, \lambda_{0}+n \delta \lambda$ the system (4.1) is integrated long enough to avoid transient effects. Once the forward propagation of $\lambda$ is complete, the coupling is adiabatically decreased until $\lambda=\lambda_{0}$ and the evolution of $r$ in the whole range of $\lambda$ is obtained. In all experiments the initial networks were constructed via the BA model with 

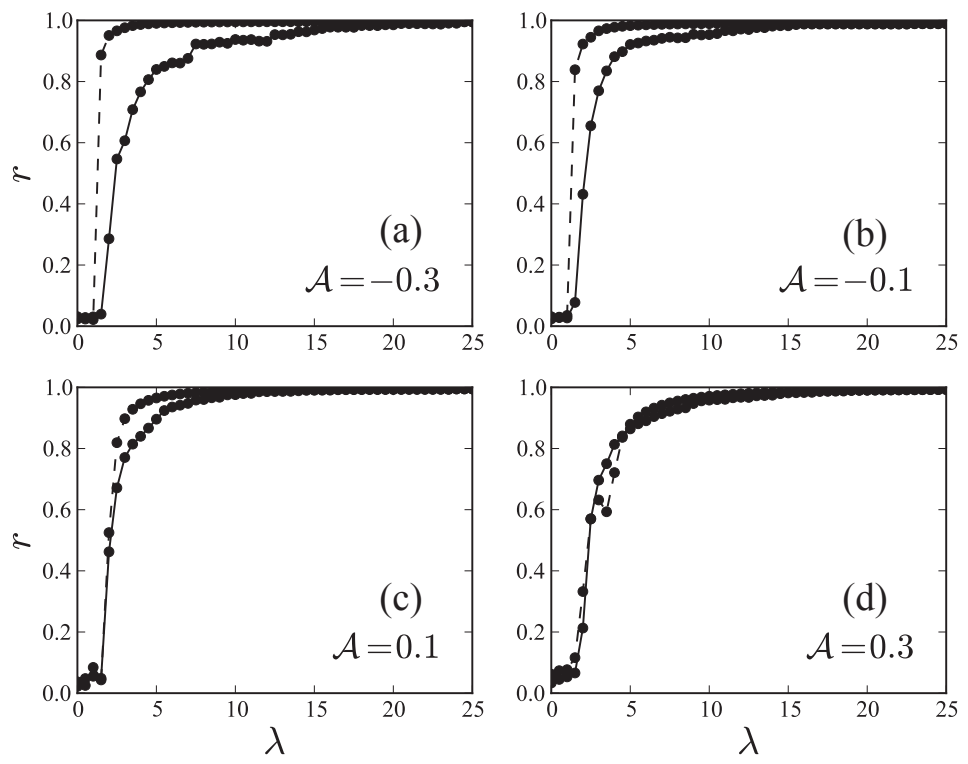

Figure 14 - Synchronization diagram $r(\lambda)$ for (a) $\mathcal{A}=-0.3$, (b) $\mathcal{A}=-0.1$, (c) $\mathcal{A}=0.1$ and (d) $\mathcal{A}=0.3$. The dissipation coefficient is fixed at $\alpha=1$ with natural frequencies given by $\Omega_{i}=k_{i}-\langle k\rangle$, as in Fig. 15. All networks considered have $N=10^{3},\langle k\rangle=6$ and $P(k) \sim k^{-\gamma}$, where $\gamma=3$.

Source: Adapted from PERON et al. (126)

$\langle k\rangle=6$ and $N=1 \times 10^{3}$.

We start our numerical experiments by fixing the dissipation coefficient $\alpha=1$ and varying the network assortativity in the interval $[-0.3,0.3]$. Fig. 14 shows the synchronization diagram $r(\lambda)$ for networks with different values of assortativity. As $\mathcal{A}$ increases, the hysteresis becomes less clear and the onset of synchronization in the decreasing branch tends to increase. Surprisingly, the critical coupling of the increasing branch for the secondorder Kuramoto model is weakly affected, in sharp contrast with results concerning models without inertia. $(130,131)$ More precisely, in the first-order Kuramoto model with frequencies correlated with degrees, the critical coupling for the onset of synchronization in SF networks occurs at higher couplings as the network becomes more assortative. $(130,131)$ The same phenomenon was observed in the synchronization of FitzHugh-Nagumo oscillators.(147) This suppression of network coherence can be linked to the fact that highly assortative networks tend to form communities - groups that are more connected withim themselves than with the rest of the population - which have nodes with similar degrees.(90) The sparse connectivity between the communities contributes to enlarge the average shortest path length which in turn ends up hindering the emergence of a macroscopic synchronous component.

Intriguingly, this characteristic of assortative networks seems to not have a significant impact on the level of synchronization in networks of second-order oscillators; only the reversibility of the phase transition suffers a noticeable influence of degree-degree 

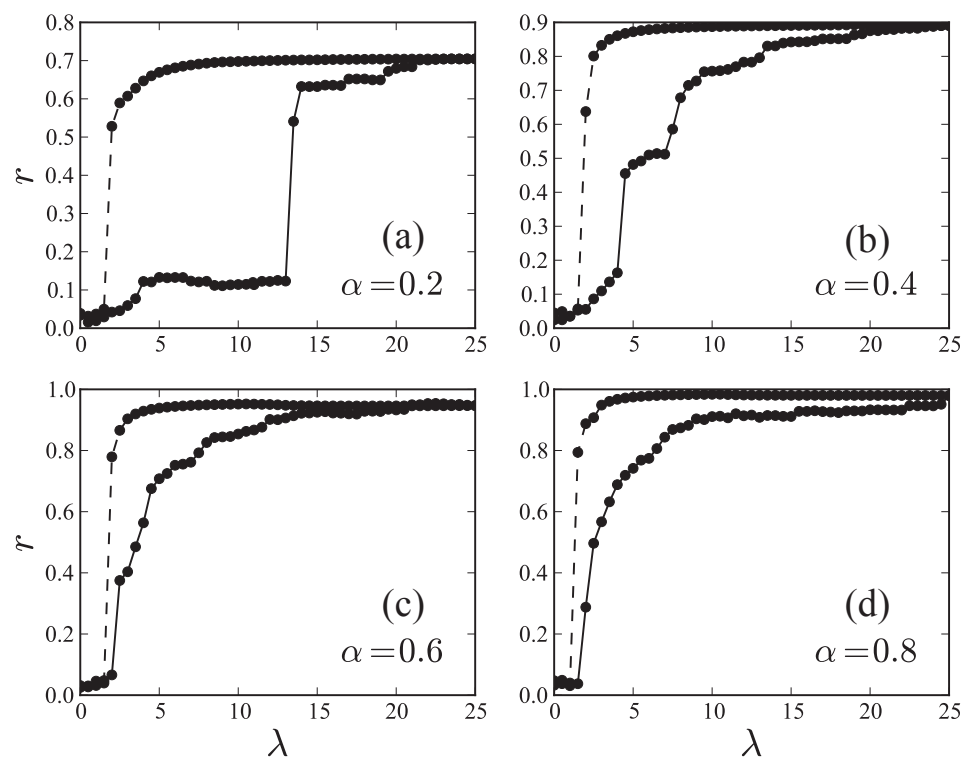

Figure 15 - Synchronization diagram $r(\lambda)$ with (a) $\alpha=0.2$, (b) $\alpha=0.4$, (c) $\alpha=0.6$ and (d) $\alpha=0.8$ for disassortativity $\mathcal{A}=-0.3$. With increasing $\alpha$, onset of synchronization and hysteresis decreases. The natural frequency of each oscillator is $\Omega_{i}=k_{i}-\langle k\rangle$ and the networks have $N=10^{3}$ and $\langle k\rangle=6$. The degree distribution follows a power-law $P(k) \sim k^{-\gamma}$, where $\gamma=3$. Curves in which points are connected by solid lines (resp. dashed lines) correspond to the forward (resp. backward) continuations of the coupling strength $\lambda$.

Adapted from PERON et al. (126)

correlations. In fact, by analyzing Fig. 14 one might conclude that positive assorativity is a synonym for the suppresion of the abrupt transition. Before jumping to this conclusion, let us investigate the situation in which the network assortativity remains fixed and another quantity related to the hysteretic behavior of the transition is varied, namely the damping parameter $\alpha$. Figure 15 shows the forward and backward curves of $r(\lambda)$ for networks with $\mathcal{A}=-0.3$, but with different values of $\alpha$ within the interval $[0.2,1]$. As we can see, the area of hysteresis and the critical coupling for the onset of synchronization in the increasing branch tend to decrease with $\alpha$, contributing also to the improvement maximal value of the order parameter.

In the network dynamics literature, when the impact of some topological property is under investigation, the usual question addressed is that whether such a property enhance or undermine the performance of a given global or local quantifier of the dynamics being studied. In many situations we can indeed provide an assertive answer to this problem. For instance, in standard formulations of the first-order Kuramoto model, positive degree-degree correlations are often related with the increase of the critical coupling for the onset of synchronization (32); assortative networks are shown to exhibit lower percolation thresholds (148) and are more robust under random nodal removal (90); the number of triangles is positively correlated with bond percolation and epidemic thresholds (149); 


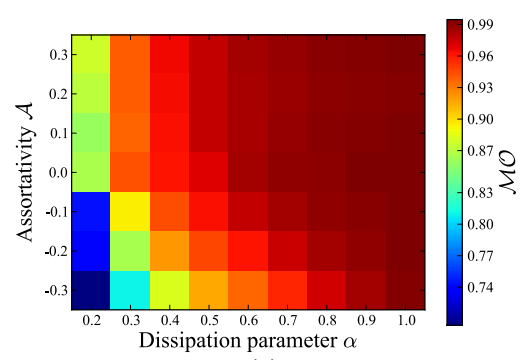

(a)

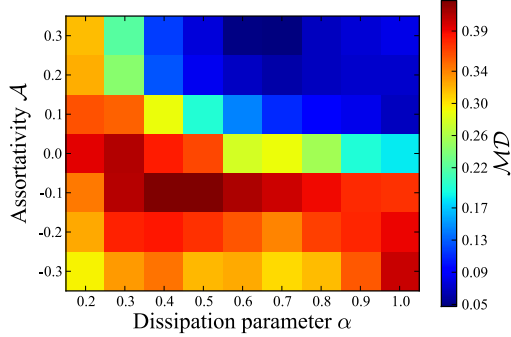

(b)

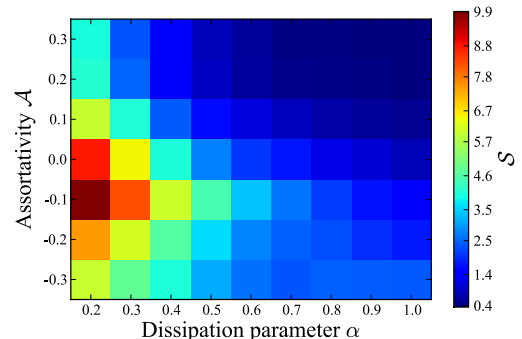

(c)

Figure 16 - Contour plot on $\alpha-\mathcal{A}$ plane colored according to (a) the maximal order parameter $\mathcal{M O}$, (b) the maximal order parameter difference $\mathcal{M D}$ and (c) the hysteresis area $\mathcal{S}$. $\mathcal{A}$ and $\alpha$ are varied within the interval $[-0.3,0.3]$ and $[0.2,1]$, respectively. For each pair $(\alpha, \mathcal{A}), 40$ times simulations are performed with the coupling strength in the interval as in Fig. 15, i.e $\lambda \in[0,25]$.

Adapted from PERON et al. (126)

and so on. However, the results in Figs. 14 and 15 do not provide a clear answer about the effects of degree-degree correlations on the dynamics of second-order oscillators; in fact, they suggest that a network might have a similar dynamical behavior if the assortativity coefficient $\mathcal{A}$ and the damping parameter $\alpha$ are properly changed. Seeking to more accurately evaluate the dependence of the synchronization transitions on the level of assortativity and the dissipation parameter, we introduce the maximal order parameter $\mathcal{M O}$, the maximal order parameter difference $\mathcal{M D}$, and the hysteresis area $\mathcal{S}$ in the synchronization diagrams as functions of $\mathcal{A}$ and $\alpha$, respectively, as follows,

$$
\begin{gathered}
\mathcal{M O}=\langle\max (r(\lambda))\rangle, \\
\mathcal{M D}=\left\langle\max \left|r^{\mathrm{I}}(\lambda)-r^{\mathrm{D}}(\lambda)\right|\right\rangle, \\
\mathcal{S}=\left\langle\int\left|r^{\mathrm{I}}(\lambda)-r^{\mathrm{D}}(\lambda)\right| d \lambda\right\rangle,
\end{gathered}
$$

where $\lambda \in\left[\lambda_{0}, \lambda_{0}+n \delta \lambda\right],\langle\cdot\rangle$ denotes the average of different realizations and $|\cdot|$ the absolute value. $r^{\mathrm{I}}(\lambda)$ and $r^{\mathrm{D}}(\lambda)$ are the order parameters for increasing and decreasing coupling strength $\lambda$, respectively. If $r(\lambda)$ increases as $\lambda$ grows, then $\mathcal{M O}$ is usually obtained at the maximal coupling strength $\lambda_{0}+n \delta \lambda$, i.e. $\mathcal{M O}=r\left(\lambda_{0}+n \delta \lambda\right) . \mathcal{M D} \in[0,1]$ quantifies the hysteresis difference. For continuous phase transitions with a perfect match between increasing and decreasing coupling branches we have $\mathcal{S} \simeq 0$. Thus, $\mathcal{S}$ is a quantitative index to evaluate the hysteretic behavior related to the emergence of synchronization.

Figure 16 shows the quantities defined in Eqs. 4.24, 4.25 and 4.26 as functions of $\alpha$ and $\mathcal{A}$ for SF networks with $P(k) \propto k^{-\gamma}$ with $\gamma=3$, as considered in Figs. 14 and 15. As it is seen in Fig. 16(a), similar values for the maximal order parameter $\mathcal{M O}$ are obtained according to the initial setup of the model, that is, the level of synchronization 

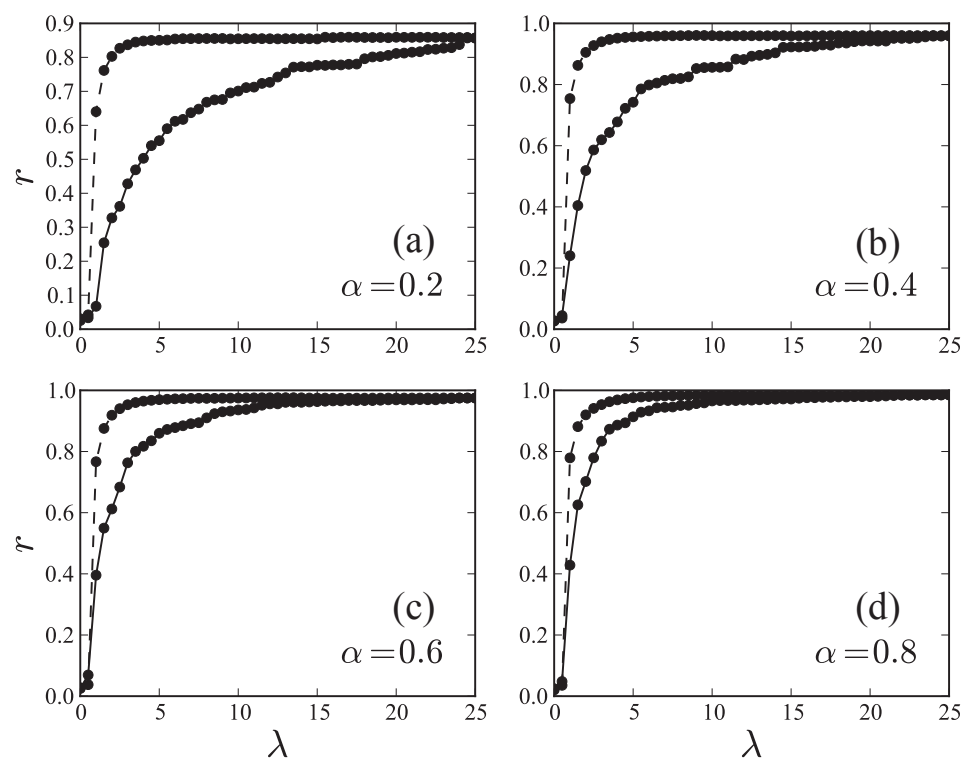

Figure 17 - Parameters are the same as in Fig. 15, except that the natural frequencies are randomly selected from a Lorentzian distribution $g(\Omega)=1 /\left(\pi\left(1+\Omega^{2}\right)\right)$.

Adapted from PERON et al. (126)

of the network can be chosen by tuning the assortativity or the dissipation parameter in the dynamical model. Therefore, for the second-order Kuramoto model in the case of frequencies positively correlated with degree, high levels of coherent behavior are obtained by either strongly assortative or dissortative networks, once the dissipation parameter $\alpha$ is properly selected. Interestingly, the maximal gap between the increasing and decreasing branches quantified by $\mathcal{M D}$ (Fig. 16(b)) has a maximum around $\mathcal{A} \simeq-0.1$ and $\alpha \in$ $[0.4,0.5]$, showing that the area of bistability in the stability diagram of the model (Fig. 9) is maximized for this set of parameters.(126)

A similar effect can also be observed for the hysteresis area $\mathcal{S}$ in Fig. 16(c). The maximal $\mathcal{S}$ in the synchronization diagram is reached for networks with $\mathcal{A}=-0.1$ and $\alpha=0.2$. Furthermore, similar values of $\mathcal{S}$ are obtained by different sets of $\alpha$ and $\mathcal{A}$, which shows an interesting interplay between the topological parameter (assortativity) and the dynamical one (damping). In other words, topological properties related to degree-degree correlations can be counterbalanced by the dissipation parameter in the dynamical model. This property could have interesting applications in the control of synchronization in network modelled by the second-order Kuramoto model. In particular, if one is interested to reduce hysteresis in a system, such a task can be accomplished by either increasing the dissipation or the degree mixing in the network. Therefore, the question usually addressed in studies regarding the first-order Kuramoto model that is whether assortativity could enhance or not synchronization $(131,150)$ turns out to be harder to answer for the damped version of the model. The reason for that is that the asymptotic behavior of the system strongly depends on the parameters $\mathcal{A}$ and $\alpha$, whose combination yields much more options 

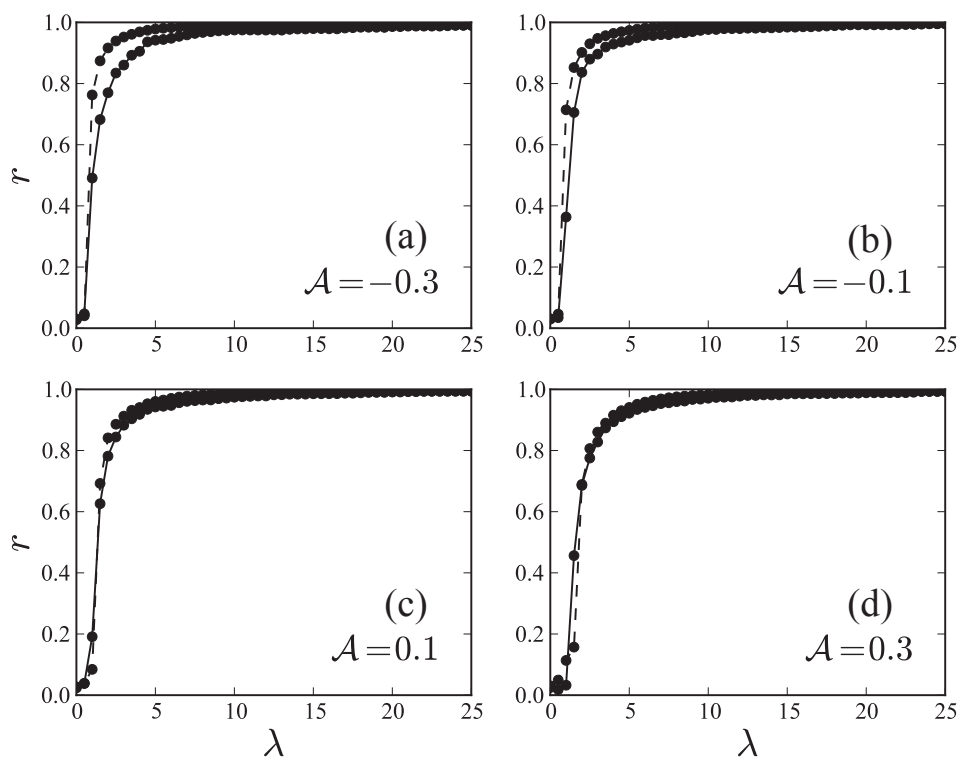

Figure 18 - Parameters are the same as in Fig. 14, except that natural frequencies are randomly selected from a Lorentzian distribution $g(\Omega)=1 /\left(\pi\left(1+\Omega^{2}\right)\right)$.

Adapted from PERON et al. (126)

to control the system's dynamics.

The results above are valid for networks with positive correlations between frequencies and degrees. It is also worth asking if the same conclusions are extendable to the standard formulation of the model in which frequencies are randomly drawn from a symmetric and unimodal frequncy distribution. Therefore, we also compute the same forward and backward synchronization diagrams considering a Lorentzian distribution $g(\Omega)=\frac{1}{\pi\left(1+\Omega^{2}\right)}$ for different values of degree assortativity $\mathcal{A}$. Similarly as before, as a first experiment, we fix the assortativity at $\mathcal{A}=-0.3$ and vary the dissipation parameter $\alpha$ as indicated in Fig. 17. Again, as we increase $\alpha$ the hysteresis area tends to decrease. The same effect is observed for a fixed $\alpha$ with varying $\mathcal{A}$, as depicted in Fig. 18.

Calculating $\mathcal{M O}$ as a function of $\alpha$ and $\mathcal{A}$ we note, however, a slight different dependence compared with the case where frequencies are proportional to degrees. As we can see in Fig. 19(a), for a fixed value of $\alpha, \mathcal{M O}$ is weakly affected by the change of the degree mixing, except for the case $\alpha=0.2$. Nonetheless, the model with frequencies correlated with degrees presents larger fluctuations for the maximum value of coherent behavior, comparing Figs. 16(a) and 19(a). On the other hand, $\mathcal{M D}$ for networks with a Lorentzian frequency distribution (Fig. 19(b)) has a similar dependence on $\alpha$ and $\mathcal{A}$ as in Fig. 16(b), though with a maximal value located at $(\alpha, \mathcal{A}) \simeq(0.2,-0.1)$. Finally, analyzing $\mathcal{S}$ in Fig. 19(c) we note the same interplay between topological perturbations in the networks - accounted by changes in assortative mixing - and dynamical features in the oscillator model characterized by the dissipation parameter $\alpha$. As shown in this 


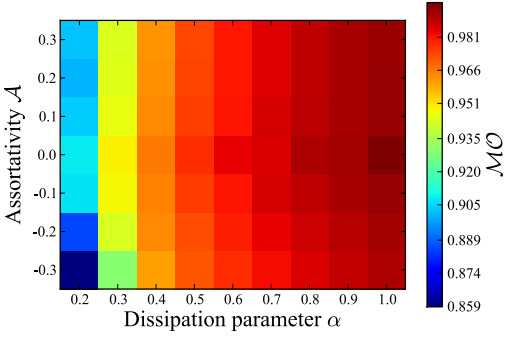

(a)

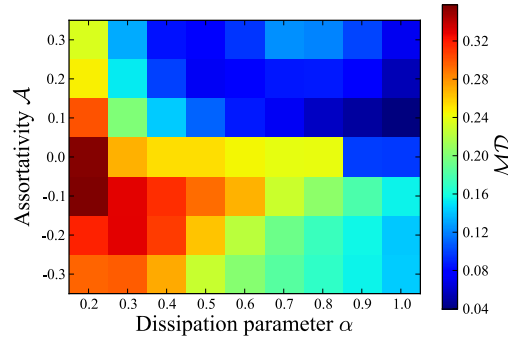

(b)

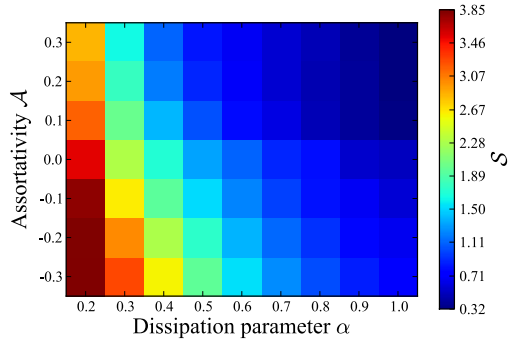

(c)

Figure 19 - Contour plots similar as in Fig. 16 for networks with a natural frequency distribution given by $g(\Omega)=1 /\left(\pi\left(1+\Omega^{2}\right)\right)$.

Adapted from PERON et al. (126)

figure, similar values of $\mathcal{S}$ are achieved by controlling the parameters $\alpha$ and $\mathcal{A}$; moreover highly and poorly hysteretic synchronization diagrams can be obtained by adopting different strategies, i.e. changing the network structure $(\mathcal{A})$ or the dynamical nature of the oscillators $(\alpha)$.

\subsection{Summary and discussions}

In this chapter we have addressed general aspects of the global dynamics of inertial phase oscillators coupled in complex networks. We started this investigation motivated by the interesting analogy that the second-order Kuramoto model offers in describing powergrid networks, systems that, as the vast majority of real networks, exihibit topological peculiarities that are not captured by traditional random graph models. Given this fact, one could be tempted to begin a work like ours directly with the analysis of the model on networks with more sophisticated topologies. However, due to the lack of general results concerning second-order phase oscillators in uncorrelated networks, we proceeded with the natural choice of first setting the theoretical basis for the latter structures. To accomplish this task we have combined the self-consistent analysis by Tanaka et al. (31) with the heterogeneous mean-field approximation presented in Sec. 2.2.1 under the special condition of having natural frequencies depending on the degrees. Our approach revealed that the order parameter indeed exhibits abrupt transitions between the asynchronous and synchronous state, however with a distinct feature from the phase transitions uncovered for the first-order model in network with similar frequency assignments. Specifically, we found that for the increasing coupling case, the synchronous network component progressively expands after the critical coupling is reached. In this process, oscillators firstly lock their phases with nodes of the same degree and then, subsequently, these groups join the synchronous component in an order from the lowest to the highest degree group [see Fig. 11 (b)]. A good agreement between the mean-field calculations and numerical results 
was obtained. We also analytically and numerically verified that the abrupt behavior of the order parameter is significantly decreased if the dependence of the natural frequencies on the degrees is disturbed by uniformly distributed quenched disorder terms. This effect is somewhat interesting because this same form of perturbing natural frequencies was found to lead to the exact opposite phenomenon in the first-order Kuramoto model, i.e. the enhancement of the discontinuous transition.

Further work should seek to meticulously clarify the nature of the phase transitions discussed in the present chapter. We remark, however, that a self-consistent analysis à la Kuramoto may not be a suitable technique to tackle the problem. As discussed in (151), the reason for this resides in the fact that the approach by Tanaka et al. (31) is not capable of providing general insights about the phase transitions for some range of parameters, especially for small values of inertia. More precisely, a fundamental limitation of the method used here is that it can predict a discontinuous phase transition even in the absence of the bistable region in the parameter space of the one oscillator dynamics. It turns out that the bistability of Eq. 4.16 only emerges after a certain critical inertia value $m_{c}$; however, very recently, Barré and Métivier (151) employed an unstable manifold expansion to prove that any non-vanishing value of inertia is able to change the nature of phase transition in the second-order Kuramoto model. Thus, we believe that any attempt that aims at rigorously characterizing the behavior of the order parameter reported here should consider approaches such as those in (151).

The influences of higher-order network properties were analyzed in terms of the variation of degree-degree correlations measured by the assortativity coefficient. Our results showed the peculiar interplay between the degree mixing in the connectivity pattern and the damping parameter with respect to the final global state of the dynamics. In particular, we observed that abrupt transitions can take place not only in dissassortative networks, but also in strongly assortative ones, in sharp contrast with the first-order oscillators coupled in networks in which the correlation between topology and dynamics is also present. The reason behind this phenomenon can be regarded as an effect of the dynamical equivalence of changes in the network structure - played by the assortative mixing - and modifications in the oscillator model (dissipation parameter). To put it differently, a given final configuration of a network of second-order Kuramoto oscillators can be achieved by tuning the network structure or by adjusting the dissipation related to the phase's movement. As previously mentioned, this property can have potential applications on controlling network synchronization in situations where, for instance, there are costs associated to lead the system to a certain desirable state, allowing one to adopt different strategies to reach this goal. One promising direction for future studies is to further analyze these results by combining the recently proposed mean-field theory for assortative networks of first-order Kuramoto oscillators (152) with the calculations presented in Secs. 2.2.2.1 and 4.1.2. 


\section{Noisy oscillators with asymmetric attractive-repulsive interactions}

In the previous chapters we have studied how the topological organization of networks affects the collective dynamics of coupled phase oscillators. Briefly recalling, in Chapter 3, the property under scrutiny was the ocurrence of cycles of order three; there our attention was centered at including building blocks of real-world networks in order to quantify how the dynamics in the latter differs from the one in idealized networks constructed by the configuration model. In Chapter 4, the driving factor for changes in the dynamics was not solely the topology itself anymore, but also variations in the oscillator model were considered, namely the presence of inertia in the phases' evolution. Despite this intrinsic difference with the respect to the damping factor, the models in both chapters had the following features in common: the interaction between the oscillators remained always attractive and only transitions from incoherence to partial synchronization were detected. It may sound odd to the reader the mere fact that we are highlighting these similarities, since it is no surprise that sufficiently strong couplings are able to bring the oscillators to an ordered state, as already shown by Kuramoto in his original paper (14). However, although the feedback between the oscillators and the mean-field is the mechanism whereby a synchronous component emerges, there is no reason to believe that this attractive interaction is the only one that mediates the dynamics of physical systems. Take, for instance, random magnetic systems such as spin-glasses (153), where the attraction and antialignment of spins give rise to ferromagnetic and glassy states; excitatory and inhibitory connections in neuronal dynamics (35); or even opinion dynamics in sociophysical models in which similar opinions tend to align in an intermediate political preference, whereas highly opposed opinions impede the system of reaching a global consensus.(36) If these aspects - i.e. different responses to the mean-field - are now incorporated in a phase oscillator model, what other collective states can one observe rather than the classical incoherent and partially synchronous configurations?

This question has actually already inspired a substantial amount of research. For instance, in his pioneer work (34), Daido established an analogy with spin-glasses by treating the couplings $K_{i j}$ between oscillators in a Kuramoto model as random variables which could be either positive or negative. His results provided evidences to an analogous glass phase transition in oscillatory systems; however, the precise conditions for the existence of these "oscillator glasses" remain elusive and still some controversy surrounds the problem.(154,155) More recently, Hong and Strogatz dedicated a series of papers (156-158) in which the role of negative and positive couplings was further exploited. In the first 
coupling setting considered by them $(156,157)$, oscillators were divided in two globally coupled populations characterized by distinct coupling strengths. In a scenario resembling sociodynamical models, oscillators within the first population have tendency to align with the mean-field, whereas the second one is constituted by oscillators that are repelled by others' influence. In the second coupling scenario (158), a portion of the ensemble provides positive coupling inputs to other nodes, while the remaining contribute with negative couplings; that is, a scheme akin to inhibitory and excitatory interactions in neuronal systems. Although very similar, the two coupling formulations yield significantly different collective dynamics. In fact, only the model in $(156,157)$ has been found to lead to different transitions other than between incoherence and classical partially synchronized states.

The work in this chapter is built upon the above mentioned contributions. Specifically, here we unify the coupling scenarios in (156-158) by considering that oscillators interact through in- and out-coupling strengths which can be either attractive or repulsive. We show that, as in (156-158), peculiar synchronous configurations in which the ensemble is split in distinct clusters are revealed; however, new routes to the various collective states are unfolded by the coupling mechanism introduced here. Futhermore, another important difference of the model presented here from Refs. (156-158) (and also from the previous chapters), is that we consider the influence of stochastic fluctuations in the phases's evolution. This feature allows us to employ the so-called Gaussian approximation $(38,159)$ whereby a reduced set of equations dictating the time-dependent behavior of global order parameters is obtained. Through this low-dimension description of the original system we derive one of our main contribution of this chapter - the bifurcation diagram between all possible collective states. Inspired by the rich dynamics uncovered, we extend the coupling mechanism conceived for phase oscillators to chaotic Rössler systems. We find that, while there is a similarity with the synchronization of Kuramoto oscillators, chaotic units diffusively coupled via repulsive and attractive interactions exhibit a strikingly different dynamics with respect to variations in the collective frequency.

It might be evident that the investigation in this chapter will not be centered on the effects of network topology on the collective phase dynamics, instead we will be interested on the role played by the nature of the interaction between the oscillators. Thus, in contrast to Chapters 3 and 4, a major part of our discussion will be focused on fully connected graphs with asymmetric attractive and repulsive couplings so that the influence of these properties is isolated from possible side effects induced by heterogeneous topologies. Nevertheless, we sketch the generalization of the problem to uncorrelated networks, leaving a more detailed analysis for future works. The next sections are organized as follows: Section 5.1 presents with more details the model addressed and Sec. 5.2.1 introduces the Gaussian approximation technique which is used to obtain the bifurcation diagrams in Sec. 5.2.2. Comparison with simulations are shown in Sec. 5.3. Having set the theoretical basis for globally coupled oscillators, in Sec. 5.4 we formulate the reduced system of 
equations for uncorrelated networks. Finally, Sec. 5.5.1 is dedicated to Rössler oscillators asymmetrically connected, followed by our final considerations in Sec. 5.6. This chapter is based on publications $(160,161)$, and the following sections will closely follow them.

\subsection{Model}

The variation of the Kuramoto model that we study here is given by $N$ stochastic coupled oscillators with the same natural frequency and whose phases evolve according to

$$
\dot{\theta}_{i}=\omega_{0}+\frac{K_{i}}{N} \sum_{j=1}^{N} G_{j} \sin \left(\theta_{j}-\theta_{i}\right)+\xi_{i}(t),
$$

where $K_{i}$ is the in- and $G_{j}$ the out-coupling strength. As defined in Eq. 5.1, these couplings make the interactions between the oscillators to be asymmetric; i.e. oscillator $i$ contributes to the dynamics of other nodes with weight $G_{i}$, but at the same time it feels the mean-field with weight $K_{i}$. The influence of stochastic forces is introduced by the terms $\xi_{i}(t)$ which are modeled as Gaussian white noise satisfying

$$
\begin{aligned}
\left\langle\xi_{i}(t)\right\rangle & =0, \\
\left\langle\xi_{i}(t) \xi_{j}\left(t^{\prime}\right)\right\rangle & =2 D \delta_{i j} \delta\left(t-t^{\prime}\right),
\end{aligned}
$$

where $D$ is the noise intensity. The physical meaning of $\xi_{i}(t)$ can be understood as rapid variations of the natural frequency or the resultant product of various stochastic processes perturbing the oscillators' phases.

\subsection{Theory}

\subsubsection{Gaussian approximation}

The Gaussian approximation is inserted in the context of a long-standing puzzle regarding the dynamics of coupled phase oscillators, namely the low-dimensional behavior of the Kuramoto model. The problem consists in finding a suitable description for the evolution of $N$ oscillators (typically in the thermodynamic limit) in terms of a finite number of ordinary differential equations. The usefulness of describing a $N$-dimensional system with just one or a few equations is tremendous; not only bifurcations can be carefully determined, but also in the practical domain, numerical solutions can be calculated much more quickly. Despite more than four decades of research, substantial progress in this subject was only achieved very recently by Ott and Antonsen.(64,162) They proposed a power-law ansatz for the Fourier coefficients of the probability distribution of phases and further showed that the whole dynamics is fully captured by a single differential equation. 
Unfortunately, the Ott-Antonsen ansatz is not applicable to noisy oscillators; however, the Gaussian approximation provides a dimensional reduction which, althought it is not exact, it has been shown to yield accurate results in different contexts.(38, 159, 163, 164) In this section we explain the main ideas behind the latter method and subsequently we show how it is generalized to the model with asymmetric connections in Eq. 5.1.

\subsubsection{Illustration - Globally coupled oscillators}

For illustration purposes, let us first briefly demonstrate how the Gaussian approximation technique applies to the simplest case of $N$ identical oscillators globally coupled and subjected to the influence of Gaussian white noise as

$$
\dot{\theta}_{i}(t)=\omega_{0}+\frac{K}{N} \sum_{j=1}^{N} \sin \left(\theta_{j}-\theta_{i}\right)+\xi_{i}(t) .
$$

Since all oscillators are identical, we are free to set $\omega_{0}=0$ due to the rotational symmetry, without lack of generality. In the thermodynamic limit of Eq. 5.3 the system can be described by a single phase distribution $\rho(\theta, t)$ obeying the normalization condition $\int_{0}^{2 \pi} \rho(\theta, t) d \theta=1$, as previously. The time evolution of $\rho(\theta, t)$ is now described by the nonlinear Fokker-Planck equation given by

$$
\frac{\partial \rho(\theta, t)}{\partial t}=D \frac{\partial^{2} \rho(\theta, t)}{\partial \theta^{2}}-\frac{\partial}{\partial \theta}\{K R(t) \sin [\psi(t)-\theta(t)] \rho(\theta, t)\}
$$

where

$$
R(t) e^{i \psi(t)}=\int_{0}^{2 \pi} \rho(\theta, t) e^{i \theta} d \theta
$$

The distribution $\rho(\theta, t)$ is a $2 \pi$-periodic function in $\theta$, thus we can express it in terms of a Fourier series as

$$
\rho(\theta, t)=\frac{1}{2 \pi} \sum_{n=-\infty}^{\infty} \hat{\rho}_{n} e^{-i n \theta},
$$

where $\hat{\rho}_{0}=1$ and $\hat{\rho}_{-n}=\hat{\rho}_{n}^{*}$, since $\rho(\theta, t)$ is normalized and real. The inverse transform yield the coefficients

$$
\hat{\rho}_{n}=\int_{0}^{2 \pi} \rho(\theta, t) e^{i n \theta} d \theta \equiv c_{n}+i s_{n}
$$

Note that $n=1$ corresponds to the order parameter in Eq. 5.5, in fact $\left|\hat{\rho}_{1}\right|=R$. Substituting Eq. 5.6 into Eq. 5.4 we obtain the infinite chain of coupled complex-valued equations for the Fourier coefficients

$$
\frac{\dot{\hat{\rho}}_{n}}{n}=\frac{K}{2}\left(\hat{\rho}_{n-1} \hat{\rho}_{1}-\hat{\rho}_{n+1} \hat{\rho}_{-1}\right)-D n \rho_{n}, n=1,2, \cdots, \infty .
$$

Apparently there is no gain in switching the problem of $N$ phase coupled equations (5.3) to an infinite system for the Fourier coefficients $\hat{\rho}_{n}$. One could proceed by truncating 
(5.8) at a sufficiently large $n$ to obtain an approximate description of the global dynamics of Eqs.5.3. In fact, evidences show $(38,159)$ that $n=6$ already produces satisfactorily accurate results due to the rapidly decay of $\left|\hat{\rho}_{n}\right|$ as $n$ increases. However, a thorough bifurcation analysis of Eqs. 5.8 would still be difficult or even unfeasible depending on the chosen $n$. An alternative approach is to seek a closure scheme capable of reducing the dimensionality of (5.8). This can be done by approximating $\rho(\theta, t)$ to a wrapped Gaussian distribution - that is, a distribution that describes points lying in the circle - with timedependent mean $\mu(t)$ and variance $\sigma^{2}(t)$. The wrapped distribution $\rho(\theta, t)$ is obtained through $(159,165)$

$$
\rho(\theta, t)=\frac{1}{2 \pi} \sum_{l=-\infty}^{\infty} f_{l}(t) e^{-i l \theta},
$$

where $f_{l}(t)$ are the characteristic coeffiecients of the unwrapped distribution, which, for the Gaussian, are given by $f_{l}(t)=\exp \left[-\sigma^{2}(t) l^{2} / 2+i l \mu(t)\right]$. Note that $f_{l}(t)$ correspond to the Fourier coefficients in the expansion in Eq. 5.6. We can check this by substituting Eq. 5.9 into Eq. $5.7(159,165)$ :

$$
\begin{aligned}
\hat{\rho}_{n}(t) & =\frac{1}{2 \pi} \int_{0}^{2 \pi} \sum_{l=-\infty}^{\infty} e^{-\sigma^{2}(t) l^{2} / 2+i[(n-l) \theta+l \mu(t)]} d \theta \\
& =\frac{1}{2 \pi} \sum_{l=-\infty}^{\infty} e^{\sigma^{2}(t) l^{2} / 2+i l \mu(t)} \int_{0}^{2 \pi} e^{i(n-l) \theta} d \theta \\
& =e^{-\sigma^{2}(t) n^{2} / 2+i n \mu(t)} .
\end{aligned}
$$

Taking the real and imaginary part of $\hat{\rho}_{n}$ we then obtain the coefficients

$$
c_{n}(t)=e^{-n^{2} \sigma^{2}(t) / 2} \cos [n \mu(t)], s_{n}(t)=e^{-n^{2} \sigma^{2}(t) / 2} \sin [n \mu(t)] .
$$

As a consequence of Eq. 5.11, - and here resides the great advantage of the framework provided by the Gaussian approximation - all coefficients $c_{n}$ and $s_{n}$ can be explicitely obtained in terms of $c_{1}$ and $s_{1}$ as, for example, $c_{2}=c_{1}^{4}-s_{1}^{4}$ and $s_{2}=2 s_{1} c_{1}\left[s_{1}^{2}+c_{1}^{2}\right]$. Hence, substituting Eq. 5.11 into Eq. 5.8 we have that the infinite set of equation is reduced to

$$
\begin{aligned}
& \dot{c}_{1}=-D c_{1}+\frac{K c_{1}}{2}\left\{1-\left[\left(c_{1}\right)^{2}+\left(s_{1}\right)^{2}\right]^{2}\right\}, \\
& \dot{s}_{1}=-D s_{1}+\frac{K s_{1}}{2}\left\{1-\left[\left(c_{1}\right)^{2}+\left(s_{1}\right)^{2}\right]^{2}\right\} .
\end{aligned}
$$

Making the variable transformation $\left\{c_{1}, s_{1}\right\} \rightarrow\left\{\mu, \sigma^{2}\right\}$ leads to

$$
\dot{\mu}=0, \dot{\sigma}^{2}=2 D+K\left(e^{-2 \sigma^{2}}-1\right) .
$$

Recalling the correspondence between coefficient $\hat{\rho}_{1}$ and the Kuramoto order parameter $R$, by employing the variable transformation $c_{1}=R \cos \psi$ and $s_{1}=R \sin \psi$ we obtain an ODE for the evolution of $R$ :

$$
\dot{R}=\frac{R}{2}\left[K\left(1-R^{4}\right)-2 D\right], \dot{\psi}=0 .
$$


The three parametrizations in Eqs. 5.12, 5.13 and 5.14 are equivalent and consist in an approximate lower-dimensional representation of the infinite system in Eq. 5.8. The accuracy and range of validity of the Gaussian approximation applied to system (5.3) were comprehensively studied in $(159,165)$ and we refer the reader to these references for further details of the theory.

\subsubsection{Oscillators with asymmetric interactions}

Now we return to our specific problem of oscillators with asymmetric interactions of Eq. 5.1. As we shall see, the generalization of the Gaussian approximation equations to this case is derived straightforwardly from the previous section. There, the whole population of $N$ oscillators is characterized by one single distribution $\rho(\theta, t)$. Here, on the other hand, we need to coarse-grain the oscillators in populations characterized by the pairs of couplings $(K, G)$, in a similar aggregation process as it was performed in the previous chapter for heterogeneous networks. Hence, in the thermodynamic limit $N \rightarrow \infty$, the population is described by the density $\rho(\theta, t \mid K, G)$ so that $\rho(\theta, t \mid K, G) d \theta$ measures the fraction of oscillators that have phases between $\theta$ and $\theta+d \theta$ at time $t$ for a given in-coupling strength $K$ and out-coupling strength $G$. The corresponding nonlinear Fokker-Planck then reads

$$
\frac{\partial \rho(\theta, t \mid K, G)}{\partial t}=D \frac{\partial^{2} \rho(\theta, t \mid K, G)}{\partial \theta^{2}}-\frac{\partial}{\partial \theta}[K R(t) \sin (\psi-\theta) \rho(\theta, t \mid K, G)]
$$

The latter equation for $\rho(\theta, t \mid K, G)$ is coupled with the evolution of other subpopulation through the mean-field amplitude $R(t)$ and phase $\psi(t)$ which are defined as

$$
R(t) e^{i \psi(t)}=\left\langle\left\langle r_{K^{\prime}, G^{\prime}}(t) G^{\prime} e^{i \psi_{K^{\prime}, G^{\prime}}(t)}\right\rangle\right\rangle
$$

where $\langle\langle\ldots\rangle\rangle=\iint \ldots P\left(K^{\prime}, G^{\prime}\right) d K^{\prime} d G^{\prime}$ is the average over the joint distribution $P(K, G)$ of out- and in-coupling strengths, and

$$
r_{K, G}(t) e^{i \psi_{K, G}(t)}=\int_{0}^{2 \pi} e^{i \theta} \rho(\theta, t \mid K, G) d \theta,
$$

defines the mean-field quantities that measure the level of synchronization within each subpopulation. Inserting the Fourier series expansion

$$
\rho(\theta, t \mid K, G)=\frac{1}{2 \pi} \sum_{n=-\infty}^{\infty} \hat{\rho}_{n}(t \mid K, G) e^{-i n \theta}
$$

into Eq. 5.15 we obtain the corresponding hierarchical system of equations for the coefficients $\hat{\rho}_{n}(t \mid K, G)$ :

$$
\frac{\dot{\hat{\rho}}_{n}}{n}=\frac{K}{2}\left[\hat{\rho}_{n-1}(t \mid K, G)\left\langle\left\langle\rho_{1}\left(t \mid K^{\prime}, G^{\prime}\right)\right\rangle\right\rangle-\hat{\rho}_{n+1}(t \mid K, G)\left\langle\left\langle\rho_{-1}\left(t \mid K^{\prime}, G^{\prime}\right)\right\rangle\right\rangle\right]-\operatorname{Dn} \hat{\rho}_{n}(t \mid K, G) .
$$


Similarly as before, seeking a closure scheme for Eq. 5.19, we assume that in every subset of oscillators with the same pair of couplings $(K, G)$ the phase distribution $\rho(\theta, t \mid K, G)$ is a Gaussian distribution with mean $\mu_{K, G}(t)$ and variance $\sigma_{K, G}^{2}(t)$. Writing the Fourier coefficients as $\hat{\rho}_{n}(t \mid K, G)=c_{n}(t \mid K, G)+i s_{n}(t \mid K, G)$, in the thermodynamic limit $N \rightarrow \infty$, GA yields

$$
\begin{aligned}
& c_{n}(t \mid K, G)=e^{-n^{2} \sigma^{2}(t \mid K, G) / 2} \cos [n \mu(t \mid K, G)], \\
& s_{n}(t \mid K, G)=e^{-n^{2} \sigma^{2}(t \mid K, G) / 2} \sin [n \mu(t \mid K, G)] .
\end{aligned}
$$

Inserting $c_{n}(t \mid K, G)$ and $s_{n}(t \mid K, G)$ from the previous equation into Eq. 5.19 and changing the variables $\left\{c_{1}(t \mid K, G), s_{1}(t \mid K, G)\right\}$ to $\left\{r_{K, G}, \psi_{K, G}\right\}$ we obtain the system

$$
\begin{aligned}
\dot{r}_{K, G} & =\frac{1-r_{K, G}^{4}}{2} K\left\langle\left\langle r_{K^{\prime}, G^{\prime}} G^{\prime} \cos \left(\psi_{K^{\prime}, G^{\prime}}-\psi_{K, G}\right)\right\rangle\right\rangle-r_{K, G} D \\
\dot{\psi}_{K, G} & =\frac{r_{K, G}^{-1}+r_{K, G}^{3}}{2} K\left\langle\left\langle r_{K^{\prime}, G^{\prime}} G^{\prime} \sin \left(\psi_{K^{\prime}, G^{\prime}}-\psi_{K, G}\right)\right\rangle\right\rangle .
\end{aligned}
$$

Before investigating the particular case of two interconnected populations, it is instructive to analyze the linear stability of the incoherent state $\rho(\theta, t \mid K, G)=1 / 2 \pi \forall K, G, t$ for an arbitrary number of populations. To this end, let us consider small perturbations $\delta r_{K, G}$ around the $r_{K, G}=0 \forall K, G$. Setting $\psi_{K, G}=0$ without loss of generality and linearizing Eq. 5.21 yields

$$
\dot{\delta r_{K, G}}=\frac{K}{2}\left\langle\left\langle\delta r_{K^{\prime}, G^{\prime}} G^{\prime}\right\rangle\right\rangle-\delta r_{K, G} D .
$$

By multiplying the previous equation by $G$ and taking the average over the distribution $P(K, G)$, we notice that it can be rewritten in terms of perturbations of the global order parameter $\delta R=\left\langle\left\langle\delta r_{K^{\prime}, G^{\prime}} G^{\prime}\right\rangle\right\rangle$ :

$$
\dot{\delta R}=\left[-D+\frac{\langle\langle K G\rangle\rangle}{2}\right] \delta R,
$$

which leads to the critical condition

$$
D_{c}=\frac{1}{2}\langle\langle K G\rangle\rangle
$$

Thus, for noise strengths $D>D_{c}$ the whole population remains incoherent, whereas for $D<D_{c}$ the incoherent state loses its stability and a partially synchronized emerges. Remarkably, the condition in Eq. 5.24 derived using the GA coincides with the exact result obtained via adapting the derivation in $(166,167)$ to the case treated here.

In principle, one can insert any distribution $P(K, G)$ into Eq. 5.21 and study the global dynamics in terms of the collective variables. However, if we choose $P(K, G)$ to be a continuous distribution we are immediately confronted with the problem of dealing again with an infinite dimensional system of ODEs. Therefore, in the analyses that follows we will adopt that the in- and out-coupling strengths are distributed according to

$$
\mathcal{P}(K, G)=\frac{1}{2} \delta\left[(K, G)-\left(K_{1}, G_{1}\right)\right]+\frac{1}{2} \delta\left[(K, G)-\left(K_{2}, G_{2}\right)\right]
$$




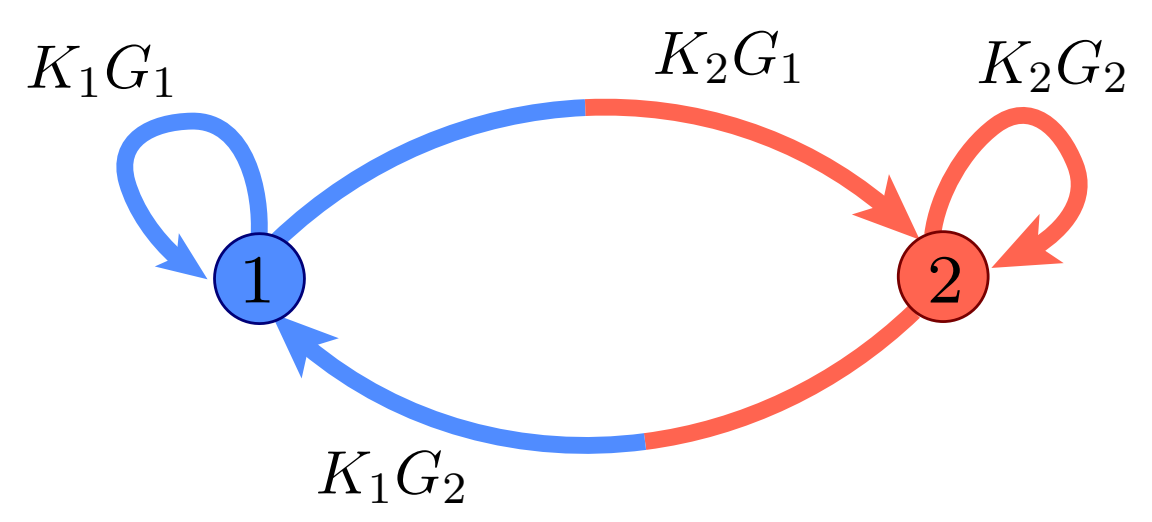

Figure 20 - Schematic visualization of the asymmetric interactions between subpopulations. Source: By the author.

That is, we consider that the whole population of $N$ oscillators is divided in two equallysized subpopulations which we shall label as " 1 " and "2". The oscillators belonging to each group are characterized by having a pair of coupling strengths, $\left(K_{1}, G_{1}\right)$ and $\left(K_{2}, G_{2}\right)$, that are allowed to assume positive and negative values. In this coupling setup, all the members of population 1 interact with themselves in symmetric fashing through the equivalent coupling strength $K_{1} G_{1}\left(K_{2} G_{2}\right.$ in population 2), while the interactions between subpopulations occur asymmetrically; nodes from population 2 receive an effective input from 1 given by $K_{2} G_{1}$, and $K_{1} G_{2}$ in the opposite direction (see Fig. 20 for an illustration).

Writing the probability densities as $\rho\left(\theta, t \mid K_{1,2}, G_{1,2}\right) \equiv \rho_{1,2}(\theta, t)$ we have that the global order parameter $R$ is reduced to

$$
R(t) e^{i \psi(t)}=\frac{1}{2}\left[r_{1}(t) G_{1} e^{i \psi_{1}(t)}+r_{2}(t) G_{2} e^{i \psi_{2}(t)}\right]
$$

where the local order parameters are now given by

$$
r_{1,2}(t) e^{i \psi_{1,2}(t)}=\int_{0}^{2 \pi} e^{i \theta} \rho_{1,2}(\theta, t) d \theta
$$

Noteworthy, $R$ is the weighted order parameter that decouples the phase equations (5.1) and it is different from the traditional Kuramoto order parameter. The latter is obtained in terms of $r_{1,2}$ by simply removing $G_{1,2}$ from $R$, i.e.

$$
r(t) \equiv \frac{1}{2}\left|r_{1}(t) e^{i \psi_{1}(t)}+r_{2}(t) e^{i \psi_{2}(t)}\right|
$$

Another key quantity of interest is the phase lag $\delta(t)=\psi_{1}(t)-\psi_{2}(t)$ that accounts for the distance between the distributions $\rho_{1,2}(\theta, t)$ in the phase axis. Rearranging Eqs. 5.21 
in terms of variables $r_{1}, r_{2}$, and $\delta$, we finally obtain

$$
\begin{aligned}
\dot{r}_{1} & =-r_{1} D+\frac{1-r_{1}^{4}}{4} K_{1}\left[r_{1} G_{1}+r_{2} G_{2} \cos \delta\right], \\
\dot{r}_{2} & =-r_{2} D+\frac{1-r_{2}^{4}}{4} K_{2}\left[r_{2} G_{2}+r_{1} G_{1} \cos \delta\right], \\
\dot{\delta} & =-\frac{\sin \delta}{4}\left[\left(r_{1}^{-1}+r_{1}^{3}\right) K_{1} r_{2} G_{2}+\left(r_{2}^{-1}+r_{2}^{3}\right) K_{2} r_{1} G_{1}\right] .
\end{aligned}
$$

The four dynamical states expected to appear in system (5.29) can be summarized as follows: (i) The first one is the known incoherent state in which $r=r_{1,2}=0$. (ii) The also already familiar partially synchronized state is characterized by $r_{1,2}>0$ and $\delta=0$ (and consequentely $r>0$ ). Henceforth we will use the term "zero-lag sync" when referring to this state. (iii) The so-called $\pi$-state is a partially synchronized state in which $r_{1,2}>0$, however the subpopulations are diametrically opposed in the phase space $(\delta=\pi)$ yielding $r=0$. In other words, local synchronization is observed within the groups but in the global scale the oscillators are observed to rotate incoherently. (iv) At last, in the traveling wave state the subpopulations are once again partially synchronized, $r_{1,2}>0$, but the phase-lag freezes in a value within $0<\delta<\pi$. The peculiar feature of this state is that, in the longtime limit, the oscillators no longer rotate with their natural frequency $\omega_{0}$, instead they settle on a new rhythm different from the intrinsic one. This behavior is unlike all collective states that we have seen so far in this thesis. Specifically, in standard formulations of the Kuramoto model, when the system approaches a partially synchronized state, oscillators become locked in a frequency close to the mean $\bar{\omega}=\int \omega g(\omega) d \omega$, where $g(\omega)$ is the natural frequency distribution. Visualizing the phases evolution in the co-rotating frame $\theta(t)-\psi(t)$, one would see that the phase distribution remains static along the phase axis. Conversely, in the traveling wave state, the peaks of the distributions $\rho_{1,2}(\theta, t)$ are free to move across the whole phase range. We quantify this spontaneous drift in the frequencies measuring the wave speed as

$$
\Omega=\frac{1}{N} \sum_{i=1}^{N}\left\langle\dot{\theta}_{i}\right\rangle_{t}
$$

where $\langle\ldots\rangle_{t}$ is a long-time average. Thus, $\Omega$ measures how strongly the new locking frequency deviates from the natural rhythm of oscillation. In Fig. 21 we exemplify the portrait of a $\pi$ - and a traveling wave state using snapshots taken from simulations of $N=10^{5}$ oscillators. In panel (a) the parameters calculated are $r_{1} \simeq 0.92, r_{2} \simeq 0.92$, $|\delta| \simeq \pi, r \simeq 0$ and $|\Omega| \simeq 0$, whereas in $(\mathrm{b}) r_{1} \simeq 0.71, r_{2} \simeq 0.98,|\delta| \simeq 0.77 \pi$ and $|\Omega| \simeq 14$.

Under which conditions these self-organized configurations appear and how the system transits between them is the topic of the next section. 

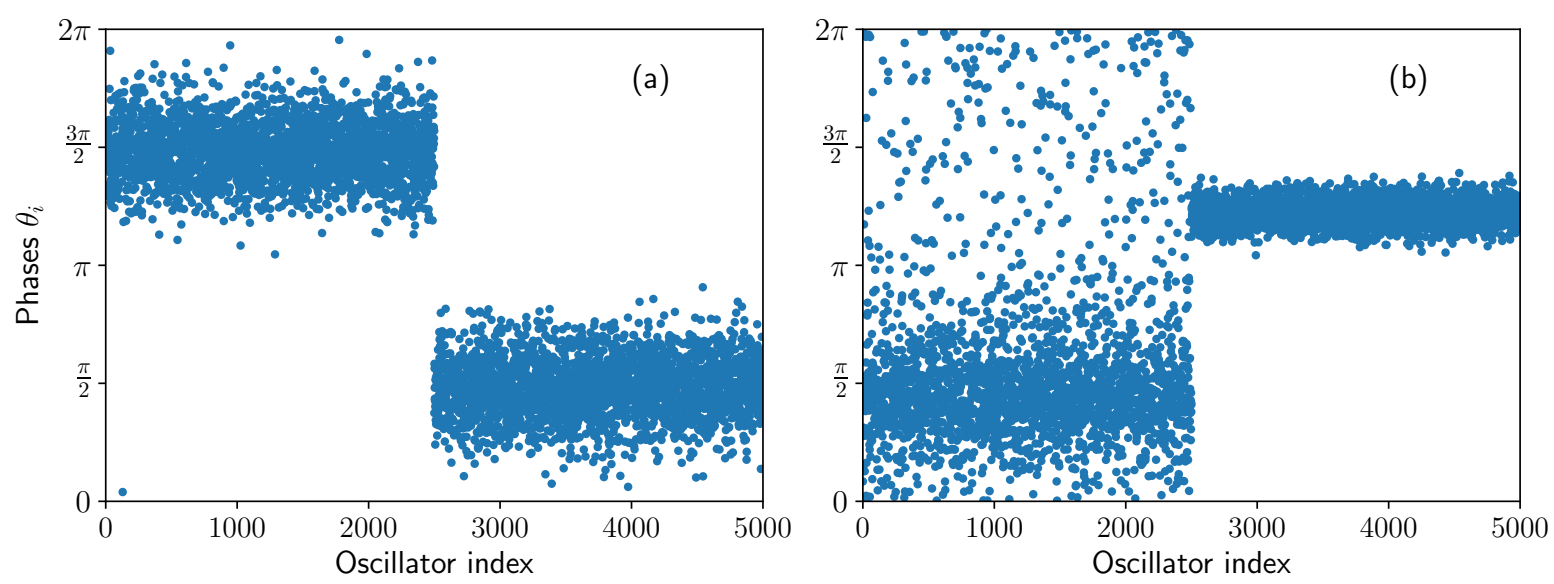

Figure 21 - Snapshots of oscillators' phases. (a) $\pi$-state with $K_{0}=0, G_{0}=0, \Delta K=8, \Delta G=2$ yielding $r_{1} \simeq 0.92, r_{2} \simeq 0.92,|\delta| \simeq \pi, r \simeq 0$ and $|\Omega| \simeq 0$. (b) Traveling wave state at $K_{0}=1, G_{0}=18, \Delta K=8$ and $\Delta G=2$ leading to $r_{1} \simeq 0.71, r_{2} \simeq 0.98,|\delta| \simeq 0.77 \pi$ and $|\Omega| \simeq 14$.

Source: By the author.

\subsubsection{Bifurcation diagrams}

All the above mentioned states are fixed point solutions of the reduced threedimensional system in Eq. 5.27. In this way, setting $\dot{r}_{1,2}=0$ we obtain two coupled equations that determine the local order parameters:

$$
\begin{aligned}
& r_{1}=\frac{r_{2}}{G_{1} \cos \delta}\left[\frac{4 D}{\left(1-r_{2}^{4}\right) K_{2}}-G_{2}\right], \\
& r_{2}=\frac{r_{1}}{G_{2} \cos \delta}\left[\frac{4 D}{\left(1-r_{1}^{4}\right) K_{1}}-G_{1}\right] .
\end{aligned}
$$

When setting $\dot{\delta}=0$ we uncover two possible fixed point solutions for the phase-lag:

$$
\begin{aligned}
\delta & =n \pi, n \in \mathbb{Z}, \\
0 & =\left(r_{1}^{-1}+r_{1}^{3}\right) K_{1} r_{2} G_{2}+\left(r_{2}^{-1}+r_{2}^{3}\right) K_{2} r_{1} G_{1} .
\end{aligned}
$$

Specifically, Eq. 5.32 corresponds to the $\pi$-state solution, while Eq. 5.33 yields the existence condition for traveling waves. This latter fact can be verified by rewriting the third equation of system (5.29) in terms of $\psi_{1}$ and $\psi_{2}$ to obtain, together with Eq. 5.33,

$$
\lim _{t \rightarrow \infty} \dot{\psi}_{1}=\lim _{t \rightarrow \infty} \dot{\psi}_{2}=\sin \delta \frac{r_{2}^{-1}+r_{2}^{3}}{4} K_{2} G_{2} r_{1}
$$

Note that now it is explicitly revealed the intrinsic relation between the phase $\delta$ and the spontaneous drift in the frequencies. In effect, traveling waves take place if, and only if, $0<\delta<\pi$; otherwise the bulk of oscillators rotates with frequency $\dot{\psi}_{1,2}=\omega_{0}$, which 
here is considered to be $\omega_{0}=0$. With Eqs. 5.31-5.33 we then obtain a system with three equations which can be solved numerically to yield the long-time behavior of $r_{1,2}$ and $\delta$.

For convenience, we henceforth adopt the following parametrization

$$
K_{1,2}=K_{0} \pm \frac{\Delta K}{2} \text { and } G_{1,2}=G_{0} \pm \frac{\Delta G}{2}
$$

where $K_{0}$ and $G_{0}$ are the average in- and out-coupling strengths, whereas $\Delta K$ and $\Delta G$ are the corresponding coupling mismatches. If $|\Delta K| / 2>K_{0}$ or $|\Delta G| / 2>\left|G_{0}\right|$ half of the couplings is positive (attractive) and half is negative (repulsive). In cases in which these conditions hold we say that the oscillators are coupled through mixed interactions.

We can use Eq. 5.24 to derive the first critical condition for the system of two subpopulations asymmetrically connected. In terms of Eq. 5.35, the incoherent state loses linear stability precisely at

$$
(\Delta K \Delta G)_{c_{1}}=8 D-4 K_{0} G_{0}
$$

Analyzing Eq. 5.31 we further notice the presence of singularities when $K_{1,2}=0$ or

$$
\Delta K_{c_{1}}= \pm 2 K_{0}
$$

Such situations correspond to the network configuration in which oscillators of one subpopulation are completely deprived of receiving inputs from other nodes (including from the same subpopulation) and have instead only out-couplings towards nodes external to their group. Furthermore, when one of the out-couplings vanishes, $G_{1,2}=0$, the oscillators of one population cease influencing the dynamics of the rest network and start acting only as link receivers. As we will shortly see, these out-couplings values play a special role in the bifurcation diagram of the system in Eq. 5.29.

So far we have obtained two exact analytical conditions (Eq. 5.36 and 5.37) related to the stability of the incoherent state and a special case of the coupling setup. But still, neither of them provide clear information about the appearance $\pi$-states and traveling waves. One way to proceed would be linearizing Eqs. 5.29 around such states and derive bounds for their existence in terms of parameters in Eq. 5.35 and the noise strength $D$. The problem with this approach is that our results would be valid only for small values for the latter mentioned quantities. Alternatively, in order to obtain a comprehensive view of the dynamics of system 5.1, we numerically detect its bifurcations with the help of the software package MATCONT.(168) Figures 22(a) and (b) show the bifurcation diagrams in the planes defined by the coupling mismatches $(\Delta K, \Delta G)$ and average couplings $\left(K_{0}, G_{0}\right)$, respectively. Solid are derived from conditions (5.36) and (5.37), while dashed lines are numerically uncovered. What immediately attracts our attention in these figures is the presence of two routes to traveling wave states, namely TW1 - reached only via crossing $\pi$ states - and TW2, which is immersed in a region of zero-lag sync. Another surprising fact 

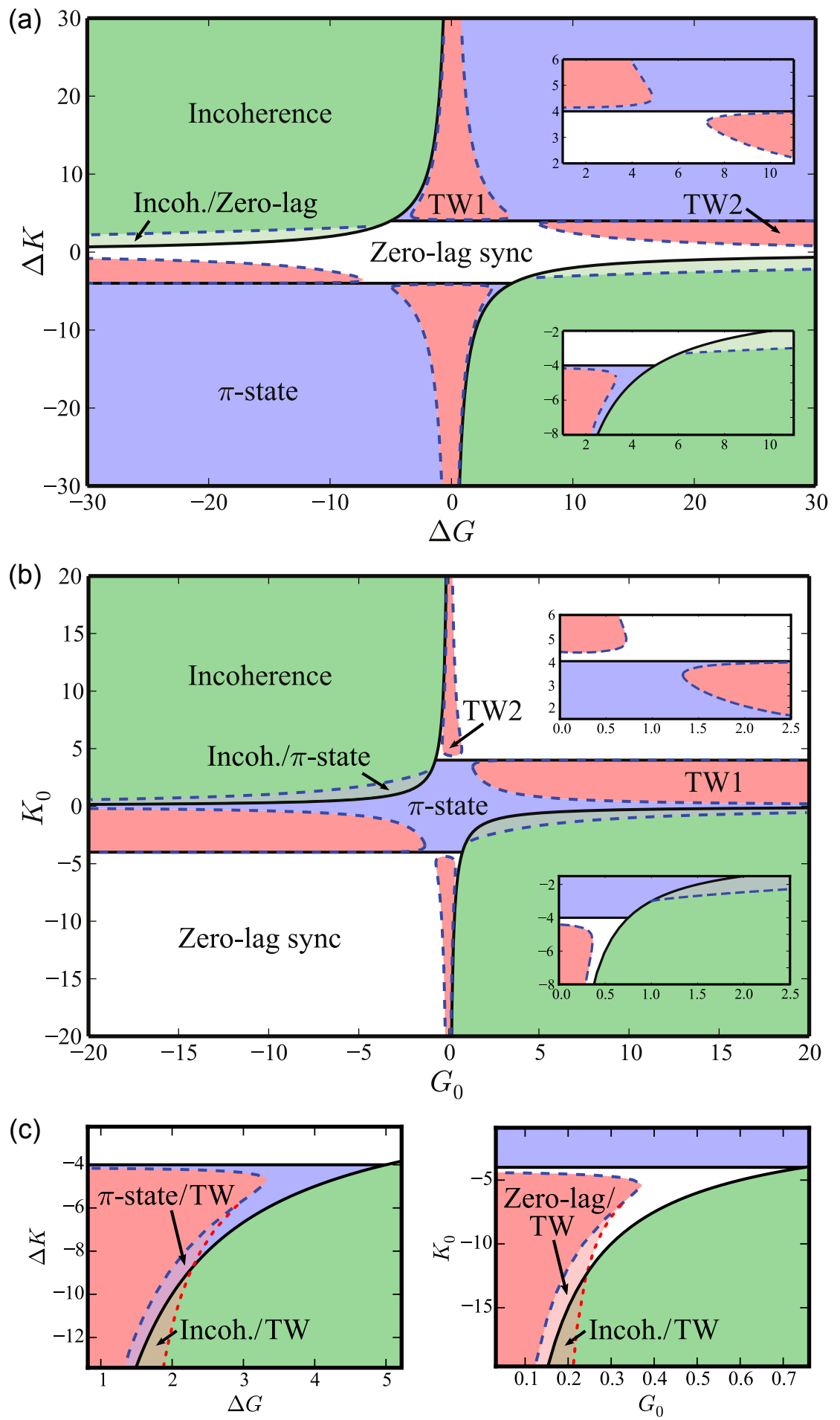

Figure 22 - Bifurcation diagrams for (a) $K_{0}=2, G_{0}=3$ and (b) $\Delta K=8, \Delta G=2$. Solid lines are derived from Eqs. 5.36 and 5.37, dashed lines obtained with MATCONT (168). "Zero-lag sync" denotes partially synchronized states with zero phase lag between the two subpopulations. Parameter regions in which $\delta=\pi$ are labeled as " $\pi$-states". Traveling wave states (TW1 and TW2) are the regions in the parameter space for which the oscillators exhibit non-zero values for the wave speed $\Omega$. (c) Zooms showing coexistence of traveling waves with other collective states. Red dotted lines (plotted only here) obtained with MATCONT.

Source: Adapted from SONNENSCHEIN et al. (160) 
is the detection of bistability between all collective states. In Fig. 22(a), we observe regions of coexistence of incoherence and zero-lag sync, while Fig. 22(b) shows the bistable region with incoherence and $\pi$-states. Figures 22(c) and (d) depicts zoomed areas of Fig. 22(a) and (b) where bistability between traveling and non-traveling wave states coexist. In fact, the points where $\Delta G= \pm 2 G_{0}$ are revealed to be the location from which the bistable regions emerge.

Interestingly, the arrangment of the bifurcation diagrams hints us some rules for the occurrence of the collective states. More precisely, in order to observe $\pi$-states, one necessarily needs to include mixed in-coupling strengths (observe that the blue areas in Fig. 22 appear only when $\Delta K>2 K_{0}$ ). Traveling waves enclosed by $\pi$-states require in turn the presence of mixed in-couplings without mixed out-couplings. On the other hand, traveling waves immersed in zero-lag sync emerge if one combines mixed out-couplings with non-vanishing mismatch in the in-couplings. Conditions for the bistable regions can be similarly derived. As it can be seen in Fig. 22(a), bistability between incoherence and zero-lag is possible only if mixed out-couplings are present jointly with non-mixed incoupling strengths. Finally, bistability between incoherence and $\pi$-states requires mixed incoupling strengths without mixing in the out-couplings. Noteworthy, these rules underline the importance of asymmetric connections for the emergence of the discussed patterns. When $K_{0} \Delta G-\Delta K G_{0}=0$ holds, the interactions become undirected. Projecting the lines defined by the latter equation onto the planes in Fig. 22, one would see that the diagrams are divided into two regions containing both TW1 and TW2; however the traveling wave zones are not intersected. Thus, asymmetric interactions are necessary conditions for the occurrence of such states. On the other hand, $\pi$-states are crossed by the lines imposed by the symmetry relation, meaning that asymmetry in the connections is not required for the observation of $\pi$-states.

Besides being interesting in its own right, the diagrams in Fig. 22 further allow us to reexamine the results by Hong and Strogatz (156-158) as particular cases of our model of asymmetric mixed connections. In the first of their series of papers (156), the authors investigated Kuramoto oscillators under attractive and repulsive interactions considering only in-couplings strengths ( $\Delta G=0$ in our notation). In this scenario, the couplings can be seen as a property of the nodes, so one speaks about "conformists" and "contrarian" oscillators. As shown in (156), the lack of out-coupling strengths does not impede the system from reaching $\pi$-states; however the transitions to traveling waves were reported to occur only via crossing $\pi$-state regions. Indeed, if we fix $\Delta G=0$ in Fig. 22(a) and follow the transitions along the $y$-axis we see that traveling waves are obtained only after passing through a $\pi$-state area. In their follow-up study (158), the couplings were regarded as properties of the links, that is, the coupling terms were considered to appear inside the summation over the neighbors' connections instead of outside as in (156). This coupling setting turns out to be equivalent to the present model in the absence of in-coupling 

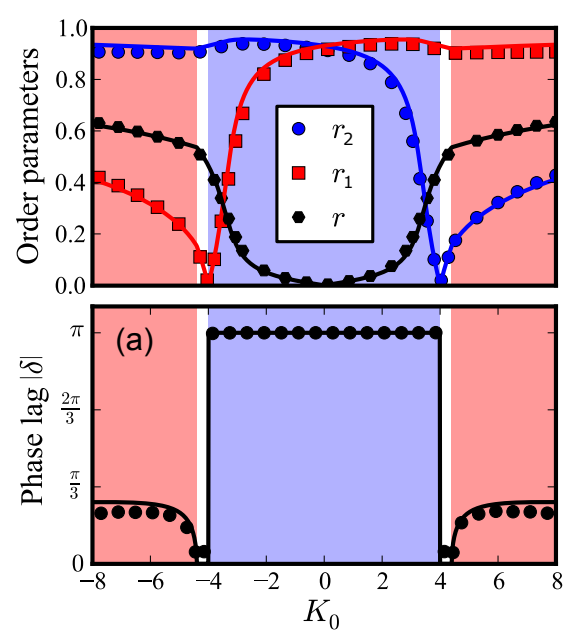
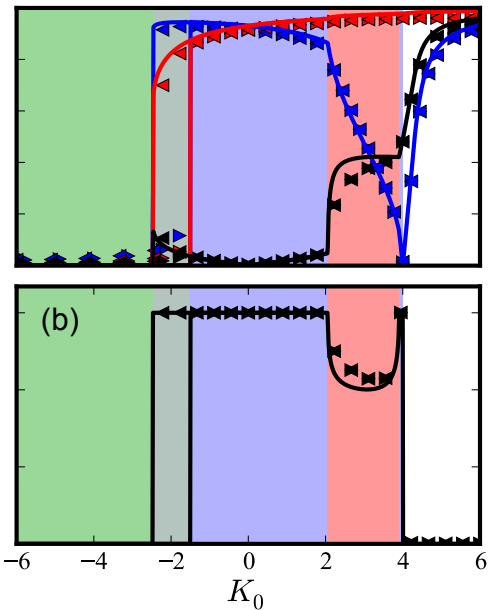
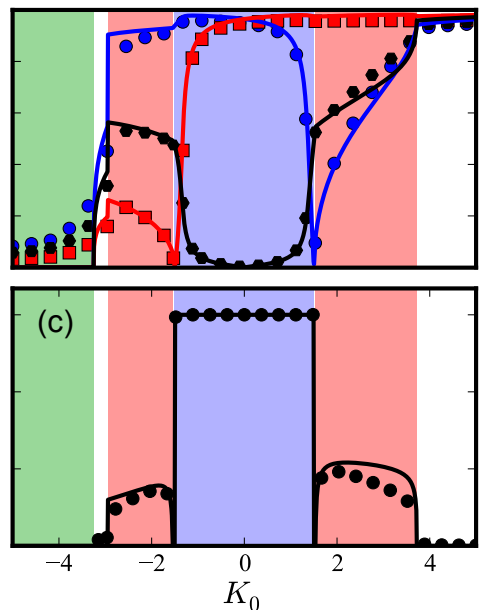

Figure 23 - Order parameters $r_{1}, r_{2}, r$ and phase lag $|\delta|$ as a function of average in-coupling strength $K_{0}$ for (a) $G_{0}=0$ and (b) $G_{0}=2$. Remaining parameters in both cases: $\Delta K=8$ and $\Delta G=2$. (c) $\Delta K=3, \Delta G=10$ and $G_{0}=2$. In all panels, dots are obtained by numerically evolving Eqs. 5.1 with $N=10^{4}$ oscillators. Solid lines are obtained by numerically solving Eqs. 5.31, 5.32 and 5.32. In order to detect the hysteresis in (b), forward $(\triangleright)$ and backward propagations $(\triangleleft)$ are performed. Shaded areas are colored according to the respective regions in the parameter spaces of Fig. 22.

Source: Adapted from SONNENSCHEIN et al. (160)

strength mismatches $(\Delta K=0)$. Surprisingly, despite the presence of mixed interactions, neither $\pi$-states nor travelings waves have been detected, but rather only transitions between the classical incoherent and partially synchronized state. Analyzing diagram in Fig. 22(a) we see that for $\Delta K=0$ only zero-lag sync is accessible by the oscillators, in agreement with the findings in (158). Observe also how close Hong and Strogatz were in detecting the TW2 zone in their second model; for extreme values of $\Delta G$, traveling waves surrounded by zero-lag sync can be reached uppon the inclusion of small in-coupling mismatch $\Delta K$.

\subsection{Simulations}

Since all our results above were obtained through an approximate reduced set of ODEs, it is therefore mandatory to ask how these findings relate with the original dynamics. Figure 23 compares the theoretical solutions obtained by solving Eqs. 5.31, 5.32 and 5.32 with numerical simulations of Eqs. 5.1. Importantly, in order to correctly yield $r_{1,2}$ and $\delta$ from the former equations, one needs to specifiy the bifurcation points beforehand. For the simulations, the initial phases are drawn from the uniform distribution defined in $[-\pi, \pi]$. For each coupling $K_{0}$, the quantities of interest are averaged over $t \in[2500,5000]$ considering time step $d t=0.01$. In Fig. 23, upper panels show the evolution of the 
order parameters, while the lower panels depict the corresponding values of the phase lag. Shaded areas are colored accordingly to the regions they belong to in the parameter space in Fig. 22.

What interests us the most in Fig. 23, besides the excellent agreement between numerical and analytical results, is the curious dependence of the order parameters on coupling $K_{0}$ induced by the successive transitions between the dynamical states. For instance, in panel (a) the transition between traveling wave and $\pi$-states lead to a highly unusual non-monotonic behavior of the total order parameter $r$. The bistability found between incoherence and $\pi$-state further cause the local order parameters $r_{1,2}$ to discontinuously jump from zero to high values, as it is seen in Fig. 23(b). This particular result is interesting per se and can be framed in the context of the explosive synchronization phenomenon discussed in the previous chapter. Abrupt transitions are also depicted in Fig. 23(c); however this time not emerging as a by-product of bistability between two states. Last but not least, in all cases, we observe anomalous scaling behaviors of the order parameters $r_{1,2}$ and $r$ close to critical coupling values, in striking contrast with the traditional square-root scaling found in standard settings of the Kuramoto model. $(16,32)$

It is also worth comparing the parameter space in Fig. 22 as a whole with simulations. In Fig. 24 we superimpose colormaps for variables $r_{1,2}, r, \delta$ and $\Omega$ with samples of the theoretical bifurcation diagrams. For each of the $100 \times 100$ data points in the $G_{0} \times K_{0}$ grid, the equations of motion 5.1 are integrated considering $N=10^{4}$ oscillators and the parameters are then averaged over the time span $t \in[100,500]$ using a time step $d t=0.01$. Figures 24(a)-(c) show the local and global order parameters. As it is seen in these panels, the areas predicted as zero-lag sync in Fig. 22(b) exhibit almost perfect synchrony as measured by $r_{1,2}$ and $r$, whereas in the $\pi$-state area intermediate values of $r_{1,2}>0$ are found jointly with vanishing $r$, as predicted by the theory. Note that non-null values of the wave speed $\Omega$ are found only in the areas demarcated as TW zones in Fig. 22(b). By choosing different initial conditions one is able to verify the bistable regions as well. Figures $24(\mathrm{e})$ - $(\mathrm{g})$ show zoomed regions $(50 \times 50$ grids points in this case) in which incoherence and $\pi$-state coexist. For the simulations in these panels, the initial conditions are adopted as $\theta_{i}(0)=1 \forall i$. Using this configuration, the system collapes into a $\pi$-state instead of exhibiting incoherence as in Figs. 24(a)-(c).

Figure 25 compares the magnitude of the spontaneous drifts predicted by GA (Eq. 5.34) with simulations. In this figure, we numerically detect the traveling waves using two indicators, namely the absolute value of the locking frequency $\left|\langle\dot{\psi}\rangle_{t}\right|$ and the wave speed $|\Omega|^{1}$. Intuitively speaking, one could expect both measures to be equivalent; indeed,

1 Noteworthy, the frequency drifts can assume either signs $\pm \Omega$, meaning that the waves can travel in both directions. The solution chosen by the system will then depend on the initial conditions and on the noise realization. For this reason, in Fig. 25 we plot the two possible theoretical curves for the common frequency $\left|\langle\dot{\psi}\rangle_{t}\right|$. 

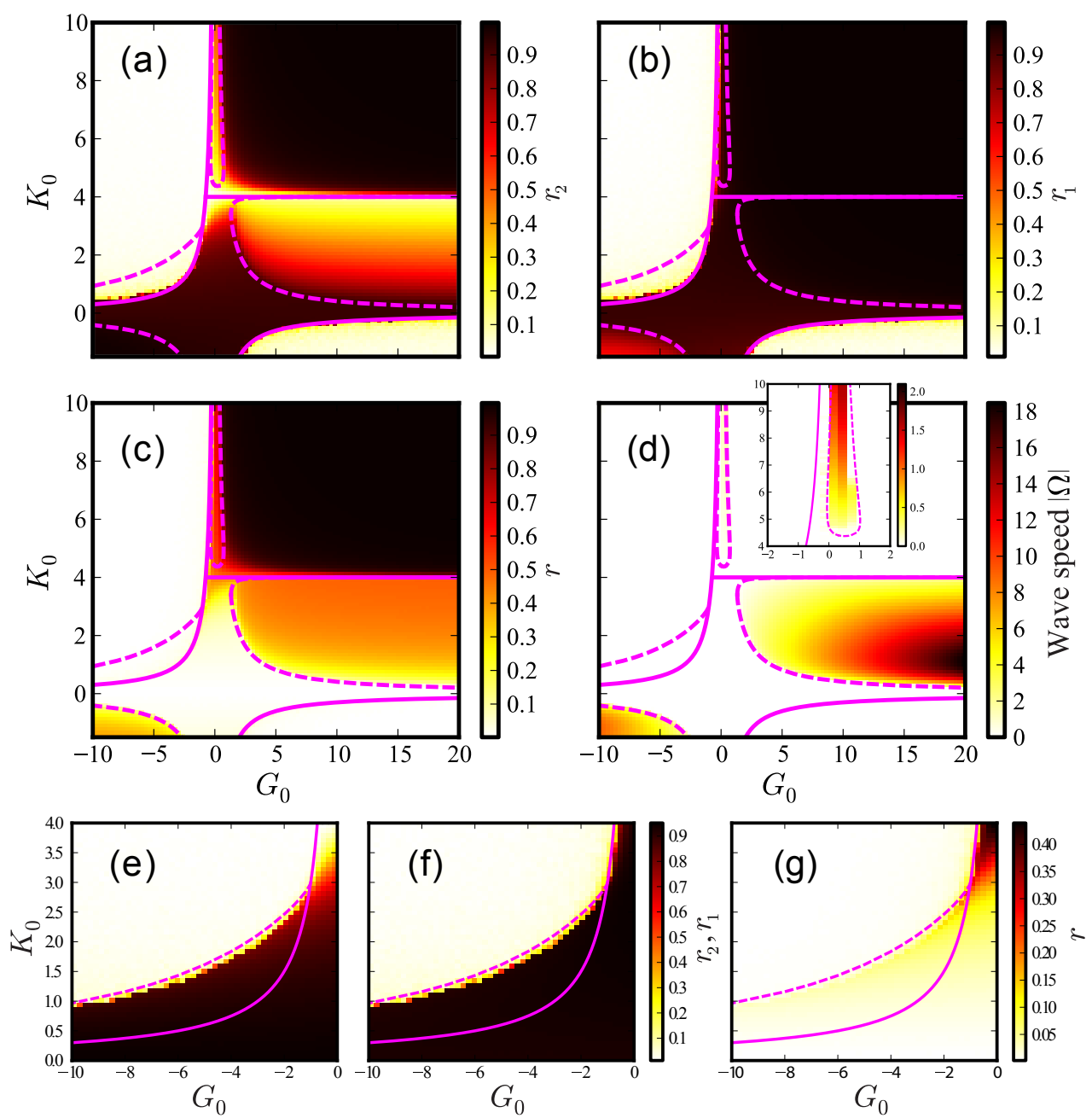

Figure 24 - Comparison between simulations (colormaps) and theory (lines). (a) and (b) depict the local order parameters $r_{2}$ and $r_{1}$, respectively; and (c) shows the global order parameter $r$. (d) Absolute value of the wave speed $|\Omega|$. Inset shows zoomed area with rescaled colormap. Simulations in panels (a)-(d) were performed with initial conditions were randomly chosen from uniform distribution defined in $[-\pi, \pi]$. In panels (e)-(g) simulations are repeated with initial conditions as $\theta_{i}(0)=1 \forall i$. Remaining parameters are the same as in Fig. 22(b).

Source: Adapted from SONNENSCHEIN et al. (160) 

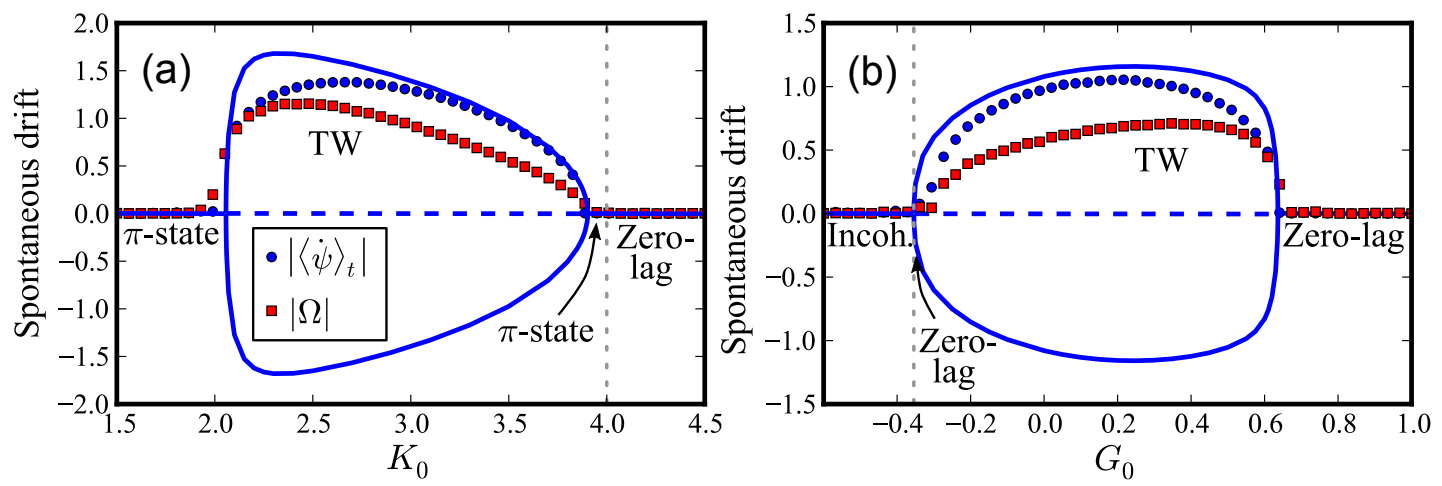

Figure 25 - Spontaneous drift measured by the the locking frequency $\left|\langle\dot{\psi}\rangle_{t}\right|$, Eq. 5.34 (theory [lines] vs. simulation [dots]) and wave speed $|\Omega|$, Eq. 5.30 only simulation) .

Source: Adapted from SONNENSCHEIN et al. (160)

in the mostly studied cases in which the frequency distributions are symmetric, $\Omega$ and $\dot{\psi}$ are used interchangeably, however in more general scenarios the equality $\Omega=\dot{\psi}$ does not necessarily hold.(169) There is, of course, a certain correspondence between them; in fact, the mean-ensemble frequency is always bounded to $|\Omega| \leq|\dot{\psi}|$. This is due to the fact that $\Omega$ is a microscopic average over the instantaneous frequencies $\left\langle\dot{\theta}_{i}\right\rangle_{t}$, while $\dot{\psi}$ quantifies how fast the center of the bulk formed by entrained oscillators moves. Thus, nodes that are not locked with the mean-field contribute to the sum in Eq. 5.30 with $\left\langle\dot{\theta}_{i}\right\rangle_{t} \approx 0$, reducing, in this way, the value of $\Omega$ in comparison with its upper bound $\dot{\psi}$. The latter frequency offers in the present case the slight advantage of being easily calculated from the fixed point solutions of the reduced system (5.29). Analogously, $\Omega$ can have its theoretical value estimated within the GA framework (not shown here) through the ensemble average

$$
\Omega=\int_{-\pi}^{\pi} \iint \dot{\theta} \rho(\theta, t \mid K, G) d K d G d \theta
$$

As shown in Fig. 25, despite these differences, the onset of the traveling wave state is equally captured by the two measures. In Sec. 5.5.1, however, we will see an example of a particular type of traveling wave that is detectable only via $\Omega$.

\subsection{Towards complex networks with attractive and repulsive asym- metric connections}

In this section we give a flavour of what to expect if we replace the fully connected graph by more heterogeneous topologies such as those considered in Chapter 3 and 4 while keeping the asymmetric mixed interactions. Introducing the connectivity pattern in 
Eq. 5.1 we arrive at the general model

$$
\dot{\theta}_{i}=\omega_{0}+K_{i} \sum_{j=1}^{N} A_{i j} G_{j} \sin \left(\theta_{j}-\theta_{i}\right)+\xi_{i}(t) .
$$

Note that we have removed the normalization factor $N$ from the coupling function in order to be consistent with our previous investigations. To apply the GA technique we must coarse-grain the network into groups of nodes with same topological and coupling parameters. In fact, here is the point in this thesis where all approaches employed thus far are considered altogether, that is, we combine the heterogeneous degree mean-field to treat the networks in the thermodynamic limit with the framework provided by the GA scheme to finally obtain an approximate reduced representation of the system in Eq. 5.39. Applying the former allows us to describe the oscillators in terms of distributions $\rho(\theta, t \mid K, G, k)$, where $\rho(\theta, t \mid K, G, k) d \theta$ measures the probability of having oscillators with phases between $\theta$ and $\theta+d \theta$ at time $t$ for a given in-coupling strength $K$, out-coupling strength $G$ and degree $k$. A nonlinear Fokker-Planck analogous to Eq. 5.15 couples the evolution of the different $\rho(\theta, t \mid K, G, k)$ via the new order parameter that accounts the degree heterogeneity:

$$
R(t) e^{i \psi(t)}=\frac{\left\langle\left\langle\left\langle k^{\prime} G^{\prime} r_{K^{\prime}, G^{\prime}, k^{\prime}} e^{i \psi_{K^{\prime}, G^{\prime}, k^{\prime}}(t)}\right\rangle_{k}\right\rangle\right\rangle}{\langle k\rangle},
$$

where $\langle\ldots\rangle_{k}=\sum_{k}^{\prime} \ldots P\left(k^{\prime}\right)^{2}$. The local order parameters are redefined as

$$
r_{K, G, k}(t) e^{i \psi_{K, G, k}(t)}=\int_{0}^{2 \pi} e^{i \theta(t)} \rho(\theta, t \mid K, G, k) d \theta .
$$

Considering $\rho(\theta, t \mid K, G, k)$ to be Gaussian distributed with time-dependent mean $\mu(t \mid K, G, k)$ and variance $\sigma^{2}(t \mid K, G, k)$ and following the calculations in Sec. 5.2, we obtain the reduced system for an uncorrelated network with in- and out-coupling strengths:

$$
\begin{aligned}
\dot{r}_{K, G, k} & =\frac{1-r_{K, G, k}^{4}}{2\langle k\rangle} K k\left\langle\left\langle\left\langle r_{K^{\prime}, G^{\prime}, k^{\prime}} G^{\prime} k^{\prime} \cos \left(\psi_{K^{\prime}, G^{\prime}, k^{\prime}}-\psi_{K, G, k}\right)\right\rangle_{k}\right\rangle\right\rangle-r_{K, G, k} D \\
\dot{\psi}_{K, G, k} & =\frac{r_{K, G, k}^{-1}+r_{K, G, k}^{3}}{2\langle k\rangle} K k\left\langle\left\langle\left\langle r_{K^{\prime}, G^{\prime}, k^{\prime}} G^{\prime} k^{\prime} \sin \left(\psi_{K^{\prime}, G^{\prime}, k^{\prime}}-\psi_{K, G, k}\right)\right\rangle_{k}\right\rangle\right\rangle,
\end{aligned}
$$

The linear stability analysis of the incoherent state $\delta r_{K, G, k}=0 \forall K, G, k$, yields that the transition to synchronization occurs at

$$
\langle\langle K G\rangle\rangle_{c}=2 D \frac{\langle k\rangle}{\left\langle k^{2}\right\rangle}
$$

Comparing the equation above with Eq. 5.24 we notice that the critical coupling for the onset of coherent behavior in the network case is simply given by the result for the

2 In contrast to the previous chapters, we are now treating the degrees as discrete variables, since the number of ODE's of the reduced system is two times the number of coarse-grained subpopulations. 
fully connected graph rescaled by the topological factor $\langle k\rangle /\left\langle k^{2}\right\rangle$, in consonance with the exposition in Sec. 2.2.1. Assuming that only two pairs of couplings are possible and are distributed according to Eq. 5.25, we generalize the reduced system (5.29) to

$$
\begin{aligned}
\dot{r}_{1, k}= & \frac{1-r_{1, k}^{4}}{4\langle k\rangle} K_{1} k\left\langle r_{1, k^{\prime}} G_{1} k^{\prime} \cos \left(\psi_{1, k^{\prime}}-\psi_{1, k}\right)+r_{2, k^{\prime}} G_{2} k^{\prime} \cos \left(\psi_{2, k^{\prime}}-\psi_{1, k}\right)\right\rangle_{k}-r_{1, k} D \\
\dot{\psi}_{1, k}= & \frac{r_{1, k}^{-1}+r_{1, k}^{3}}{4\langle k\rangle} K_{1} k\left\langle r_{1, k^{\prime}} G_{1} k^{\prime} \sin \left(\psi_{1, k^{\prime}}-\psi_{1, k}\right)+r_{2, k^{\prime}} G_{2} k^{\prime} \sin \left(\psi_{2, k^{\prime}}-\psi_{1, k}\right)\right\rangle_{k} \\
\dot{r}_{2, k}= & \frac{1-r_{2, k}^{4}}{4\langle k\rangle} K_{2} k\left\langle r_{2, k^{\prime}} G_{2} k^{\prime} \cos \left(\psi_{2, k^{\prime}}-\psi_{2, k}\right)+r_{1, k^{\prime}} G_{1} k^{\prime} \cos \left(\psi_{1, k^{\prime}}-\psi_{2, k}\right)\right\rangle_{k}-r_{2, k} D \\
\dot{\psi}_{2, k}= & \frac{r_{2, k}^{-1}+r_{2, k}^{3}}{4\langle k\rangle} K_{2} k\left\langle r_{2, k^{\prime}} G_{2} k^{\prime} \sin \left(\psi_{2, k^{\prime}}-\psi_{2, k}\right)+r_{2, k^{\prime}} G_{2} k^{\prime} \sin \left(\psi_{1, k^{\prime}}-\psi_{2, k}\right)\right\rangle_{k},
\end{aligned}
$$

One clearly sees that in the network case the Gaussian approximation does not lead to a dimensional reduction as drastic as the one achieved in Sec. 5.2. More precisely, we derived in Eq. 5.44 a system with $4 N_{k}$ ODEs, where $N_{k}$ is the number of different degrees in the network. We should, however, not underestimate the value of this approach in view of this fact. It turns out that in many circumstances the reduced system can still be extremely useful to grasp the global dynamics such as in the cases of regular and binary networks (163), where a thorough bifurcation analysis is amenable. For practical purposes, the formulation $\left\{r_{1, k}, \psi_{1, k}, r_{2, k}, \psi_{2, k}\right\}$ further allows much more faster simulations compared to the original dynamics, since $N_{k} \ll N$. Besides, depending on the network topology under study, averages over different realizations of Eq. 5.39 must be performed in order to produce smooth data for the evolution of the order parameters - specially for couplings near the transition -, whereas one single run of the reduced system suffices.

To illustrate the impact of the coexistence of more elaborated connectivity patterns and asymmetric connections, let us consider random binary networks which provide the simplest example of a heterogeneous degree distribution. In this topology, a fraction $p$ is connected to $k_{1}$ nodes and the rest of the population has degree $k_{2}$. Thus, the degree distribution is simply given by

$$
P(k)=p \delta_{k, k_{1}}+(1-p) \delta_{k, k_{2}}
$$

Noteworthy, we are not assuming that the degrees are correlated with in- and out-coupling strengths. In other words, the subpopulation characterized by the coupling pair $\left(K_{1}, G_{1}\right)$ is allowed to posses oscillators with both degrees and the same is valid for subpopulation with couplings $\left(K_{2}, G_{2}\right)$.

In Fig. 26 we show the simulation results of the order parameters for a network with degree distribution given by Eq. 5.45. While there are many similarities with the dynamics in the fully connected graph, something strikingly different is noticed. Precisely, for negative average in-couplings, a hysteresis in the evolution of $r_{1}$ and $r_{2}$ appears. As 

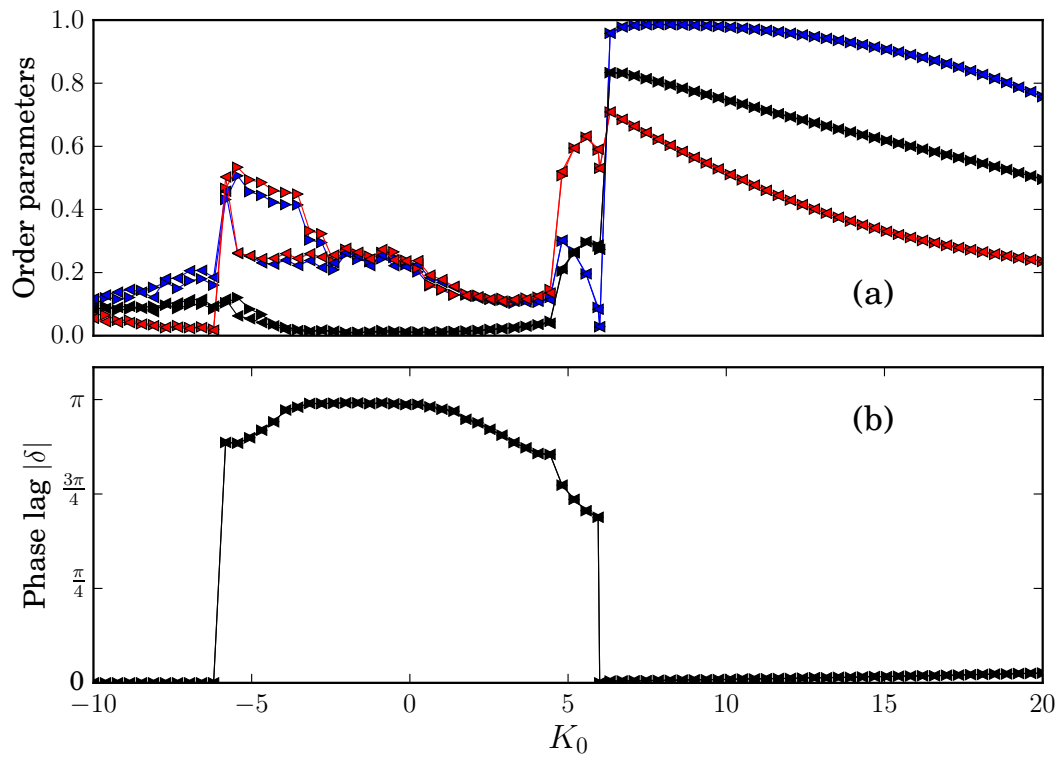

Figure 26 - (a) Order parameters $r_{1}, r_{2}$ and $r$; and (b) phase lag $\delta$ for a random binary network with degrees $k_{1}=10$ and $k_{2}=15$. Remaining parameters: $N=1000, \Delta K=12$, $\Delta G=2, G_{0}=3$ and $D=0.5$.

Source: By the author.

previously mentioned, hysteretic transitions of order parameters have been extensively reported to emerge in the synchronization of Kuramoto oscillators as a consequence of the bistability between incoherent and synchronous states. Surprisingly, this is not the case of the random binary network tackled here. What makes the phenomenon in Fig. 26 particularly special is that the hysteresis observed there is a result of the coexistence between two distinct coherent states; in the forward propagation of $K_{0}$, the oscillators collapse in a state where $r_{1,2} \approx 0.5$, whereas the same range of parameters yields $r_{1,2} \approx 0.2$ in the backward simulation. We also highlight that this intriguing behavior is manifested in a very simplistic network. What other dependencies alike the one in Fig. 26 can emerge in more sophisticated topologies is a subject that we leave for future investigations.

\subsection{Asymmetric networks of noisy chaotic attractors}

The discovery that coupled chaotic oscillators are able to synchronize their phase angles $(170,171)$ marked a milestone in the study of collective synchronization. The fascination around this phenomenon stems from the observation of phase locking among interacting chaotic units while their amplitude variables persist chaotic and uncorrelated and from each other. Interestingly, under sufficiently weak couplings, this collective phase dynamics turns out to be akin to the synchronization process of self-sustained periodic 
oscillators $(1,172)$; i.e. starting from a configuration where the phases rotate incoherently, as the coupling increases, a mean-field emerges entraining the phases and bringing the system to a partially phase synchronized state. This is somewhat surprising since, in contrast with periodic oscillators, the phases intrinsically depend on the chaotic evolution of the amplitudes. Given this unexpected similarity with Kuramoto-like models, could then the coupling setting investigated in the previous sections possibly lead to similar dynamical states in the phase dynamics of chaotic oscillators? If so, could we estimate the onset of synchronization based on conditions derived in Sec. 5.2? To answer these questions, here we will investigate two different formulations for the coupling setup between asmmetrically connected Rössler systems; namely, in the weighted $x$-coupled model, we consider the chaotic oscillators to be coupled through the $x$ coordinate, whereas in the phase-coupled model the oscillators are set to interact purely through the sine of the phase differences.

But before we delve into the collective dynamics of large ensembles of Rössler units, we need to address an issue that we have not faced when dealing with Kuramoto oscillators: How to define phases for trajectories that evolve on a chaotic attractor? These objects are usually high dimensional and this makes the search for a suitable phase and amplitude to be a highly non-trivial task whose feasibility will crucially depend on the attractor's topology.(172) A phase is simply a variable that parameterizes the motion of a given oscillator along a limit cycle. Thus, if the chaotic system exhibits periodic oscillations, a possible way of defining a phase is to look for a suitable projection of the attractor onto a plane so that it resembles a smeared limit cycle with well-defined oscillations around some coordinates. To be more concrete, let us illustrate this for the Rössler system (173)

$$
\begin{aligned}
& \dot{x}=-y-z, \\
& \dot{y}=x+a y, \\
& \dot{z}=b+z(x-c) .
\end{aligned}
$$

For $a<a_{c} \approx 0.186$ the $x y$-projection of the Rössler has a structure as depicted in Fig. 27(a). The attractor in this regime has an almost phase coherent dynamics in the sense that all trajectories oscillate around the origin $\left(x_{0}, y_{0}\right)=(0,0)$ with similar return periods. For phase-coherent attractors such as the one in Fig. 27(a) the phase associated to a given trajectory can be simply defined via transformation to the polar coordinates, i.e. $(172)$

$$
\theta(t)=\arctan \left[\frac{y(t)}{x(t)}\right]
$$

If, on the other hand, $a>a_{c}$, the topology of the attractor becomes more complex in a way that the trajectories no longer complete perfect cycles around $\left(x_{0}, y_{0}\right)$. Instead, small and large loops are created in the $x y$-plane causing the oscillator to return earlier to a certain reference point in its orbit (see Fig. 27). These "shortcuts" introduced by the funnel structure of the attractor make the phase definition in Eq. 5.47 completely 

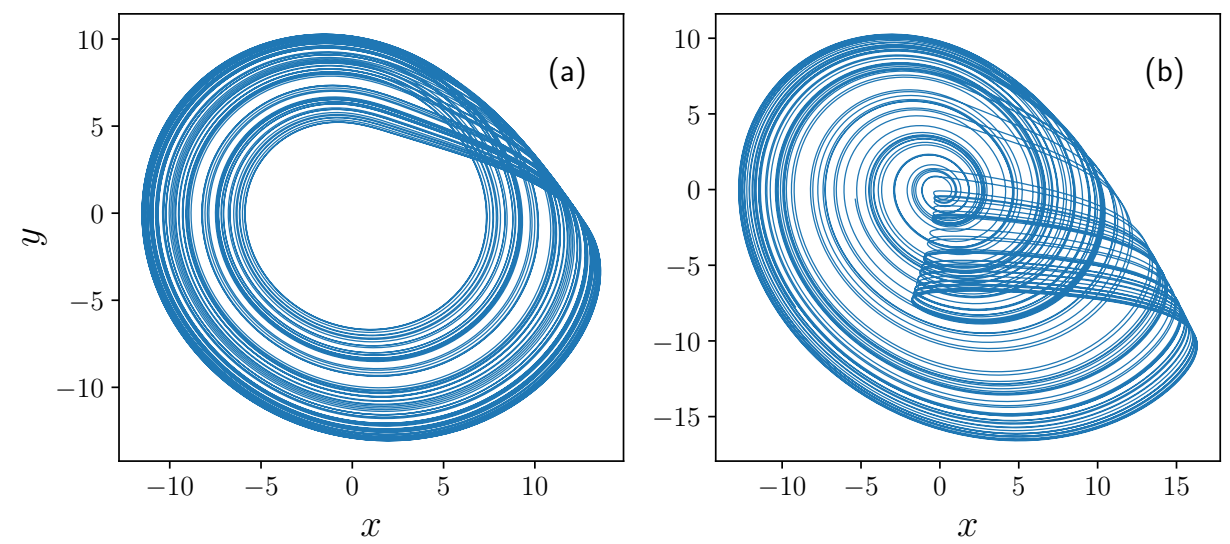

Figure 27 - Phase portrait of the dynamics of the Rössler system (Eq. 5.46) with (a) phasecoherent attractor $(a=0.15, b=0.4, c=8.5)$ and (b) funnel attractor $(a=0.2, b=$ $0.4, c=7.5)$.

Source: By the author.

inappropriate, once it is not clear how these induced phase shifts should be accounted in a polar coordinate transformation. An alternative way to define phases for any non phasecoherent attractor is found in the concept of analytic signal originally introduced by Gabor.(174) This general approach is based on the Hilbert transform and uniquely assign an instananeous phase and amplitude to a signal $s(t)$ via constructing its analytical signal as

$$
\zeta(t)=s(t)+i s_{\mathrm{H}}(t)=A(t) e^{i \theta_{H}(t)},
$$

where $s_{H}(t)$ is the Hilbert transform of $s(t)$ being calculated as

$$
s_{\mathrm{H}}(t)=\frac{1}{\pi} \int_{-\infty}^{\infty} \frac{s(\tau)}{t-\tau} d \tau
$$

where the integral is calculated in the sense of the Cauchy principal value. The instantaneous phase of $s(t)$ is then obtained through

$$
\theta_{\mathrm{H}}(t)=\arctan \left[\frac{s_{\mathrm{H}}(t)}{s(t)}\right] .
$$

To understand the idea behind this approach consider the simple case of $s(t)=\cos \left(\omega_{0} t\right)$. The Hilbert transform of $s(t)$ yields $s_{H}(t)=\sin \left(\omega_{0} t\right)$, meaning that the transformed signal is just given by the original one shifted in $-\pi / 2$. For a general signal, what the Hilbert transform effectively does is to shift every spectral component of $s(t)$ by $-\pi / 2$ creating, in this way, a signal in quadrature to $s(t)$ which, summed to it, converts the oscillating function to a rotating vector in the complex plane. Noteworthy, for phasecoherent Rössler attractors, although the phase definitions in Eqs. 5.47 and 5.50 do not match microscopically, one verifies that the average frequencies obtained through them coincide and lead precisely to the same observable phase dynamics.(1) In a experimental 
point a view, the Hilbert transform offers the advantage to be easily computed from any measureable scalar time series, not requiring the search for a suitable projection of the attractor.

In the sequel, we firstly address the problem of $N$ Rössler oscillators with phasecoherent and funnel attractors coupled through the $x$ coordinate. In this part we estimate the phases via Eq. 5.50 considering $x(t)$ as the observable time series. Subsequently, for Rössler systems coupled via their phases, the global parameters are calculated directly using the angles derived from the polar coordinate transformation. Our goal with this study is to determine whether asymmetric mixed interactions induce similar collective states as those in Secs. 5.1-5.4 in higher dimensional system with chaotic amplitude dynamics.

\subsubsection{Rössler systems with diffusive coupling}

Let us start considering the case where the oscillators interact through the $x$ coordinate. Translating the asymmetric coupling setting in Eq. 5.1 to this paradigm we have:

$$
\begin{aligned}
& \dot{x}_{i}=-\omega_{0} y_{i}-z_{i}+\frac{K_{i}}{N} \sum_{j=1}^{N} G_{j}\left(x_{j}-x_{i}\right)+\xi_{i}(t), \\
& \dot{y}_{i}=\omega_{0} x_{i}+a y_{i}, \\
& \dot{z}_{i}=b+z_{i}\left(x_{i}-c\right) .
\end{aligned}
$$

The phases are then defined via the Hilbert transform of $x_{i}(t)$, i.e.

$$
\theta_{i}^{\mathrm{H}}=\arctan \left[\frac{x_{i}^{\mathrm{H}}(t)}{x_{i}(t)}\right]
$$

with $x_{i}^{\mathrm{H}}$ being

$$
x_{i}^{\mathrm{H}}(t)=\frac{1}{\pi} \int_{-\infty}^{\infty} \frac{x_{i}(\tau)}{t-\tau} d \tau
$$

where the integral is taken in the sense of the Cauchy principal value.(175)

Similar as for the phase oscillators in the previous sections, coupling strength $K_{i}$ is responsible for how strongly node $i$ responds to the interaction with the rest of the population, whereas $G_{i}$ accounts for how strongly it influences the dynamics of other oscillators.

Having defined the phases, the level of phase synchronization is measured by the order parameter

$$
r=\frac{1}{N}\left\langle\left|\sum_{j=1}^{N} e^{i \theta_{j}^{\mathrm{H}}(t)}\right|\right\rangle_{t},
$$

where $|\cdot|$ is the absolute value and $\langle\cdots\rangle_{t}$ denotes temporal average. Similar as for the Kuramoto model analyzed in the previous section, we again divide the system into two subpopulations, organising the index labelling such that $j \in\{1, \ldots, N / 2\}$ refers to oscillators 

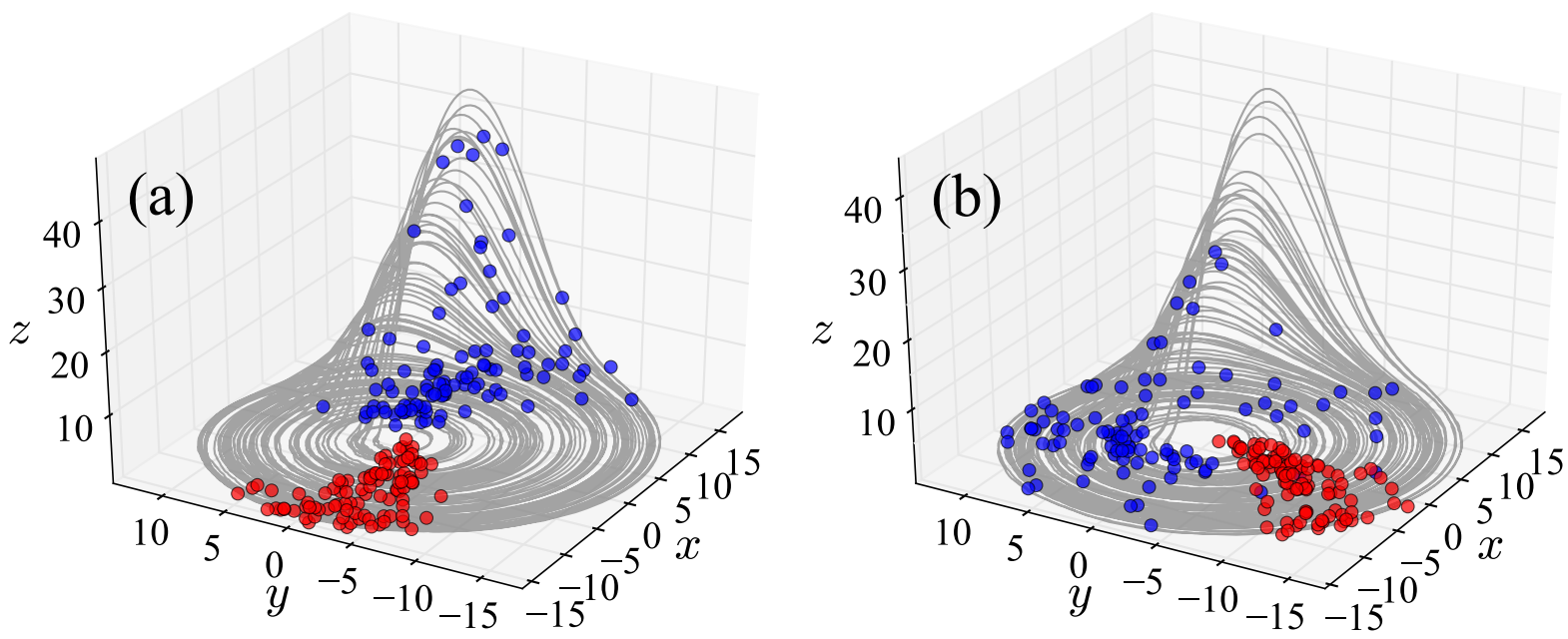

Figure 28 - Snapshots of the oscillators' trajectories of the weighted $x$-coupled Rössler model [Eq. 5.51 with phase-coherent attractor $(a=0.15, b=0.2$, and $c=10)$. (a) $\pi$-state where $\delta=\pi$ with $K_{0}=0$ and $G_{0}=0$. (b) Lag sync state for which $\delta<\pi$ with $K_{0}=0$ and $G_{0}=0.2$. Gray line in the background corresponds to a trajectory of a randomly selected oscillator in order to depict typical dynamics. Remaining parameters: $N=200, \Delta K=0.1, \Delta G=0.1$, and $D=0.03$. See the supplemental material of (161) for the animated version of the figure.

Source: Adapted from PERON et al. (161)

belonging to subpopulation 1 and $j \in\{N / 2+1, \ldots, N\}$ to subpopulation 2 . The coherence within each group is then calculated by parameters $r_{1} e^{i \psi_{1}(t)}=(N / 2)^{-1} \sum_{j=1}^{N / 2} \exp \left[i \phi_{j}^{\mathrm{H}}(t)\right]$ and analogously for $r_{2} e^{i \psi_{2}(t)}$, with $\psi_{1,2}(t)$ being the corresponding mean phases. The phase lag $\delta(t)$ is again defined as the difference between the mean phases $\delta(t)=\left|\psi_{1}(t)-\psi_{2}(t)\right|$. Moreover, the wave speed is calculated as

$$
\Omega=\frac{1}{N} \sum_{j=1}^{N}\left\langle\frac{d \theta_{i}^{\mathrm{H}}}{d t}\right\rangle_{t} .
$$

Many of the collective states that we will see below in the phase dynamics of Rössler systems are indeed equivalent to those uncovered in the beginning of this chapter for Kuramoto oscillators. This is already an interesting finding, since it shows that, despite the chaotic evolution of the amplitudes, a high dimensional system can resemble in global aspects a much more simple dynamics in the absence of chaos. However, fundamental differences arise concerning the emergence of traveling waves. In order to systematically compare the phase dynamics of Kuramoto and Rössler oscillators, in the following we summarize the results to come by stating the self-organized configurations attainable by the latter: (i) In the incoherent state, oscillators of both populations rotate independently so that $r_{1,2} \approx 0$. (ii) The oscillators are mutually attracted in the partial phase synchronized state yielding $r>0$ and $\delta=0$ which we denominate as "zero-lag sync", as previously. (iii) A similar state to (ii) is observed in system (5.51). Specifically, we refer to "lag sync" 
when partial global synchronization is achieved $(r>0)$ with $0<\delta<\pi$ in the absence of additional drift $\left(\left|\Omega-\omega_{0}\right| \simeq 0\right)$. The reader may recall that this description almost coincides with the definition of the traveling wave state in Sec. 5.2.2; however, as we shall see, non-vanishing values of $\delta$ no longer necessarily predict the emergence of spontaneous drifts. Instead, we will only refer to (iv) traveling wave states in cases where $\left|\Omega-\omega_{0}\right|>0$, regardless of other parameters. (v) Finally, in the $\pi$-state oscillators belonging to the same subpopulations are partially synchronized in phase $\left(r_{1,2}>0\right)$, while the mean phases are separated by $\delta=\pi$. Figure 28 depicts snapshots of the trajectories of $N=200$ weighted $x$-coupled Rössler oscillators (Eq. 5.51). Precisely, Fig. 28(a) shows a typical long-term configuration of a $\pi$-state, where the populations are diametrically opposed. A typical lag sync state in the weighted $x$-coupled Rössler model is exemplified in Fig. 28(b), where the centroids of the two clusters are separated by a phase-lag $\delta<\pi$.

Before continuing, we should remark on the possibility of facing trajectories that escape the attractor after a period of transient chaos. Divergent trajectories arise due to the dissipative character of the Rössler dynamics and are in general related to the conversion of a chaotic attractor into a nonattracting chaotic invariant set. $(176,177)$ In order to show how this is manifested with weighted $x$-coupled Rössler oscillators in the absence of noise, Fig. 29 depicts a sample of the plane $G_{0} \times K_{0}$ where trajectories that divergo to infinity are possible. For large values of $\left|G_{0} K_{0}\right|$, divergences from the attractor are inevitable, but still a wide region with nondivergent orbits is observed. Noteworthy, the collective escapes are induced by the coupling, since isolated oscillators do not escape for the parameters considered here as shown in Refs. $(176,177)$.

Taking Fig. 29 as a guide so that the parameters are chosen in a region free from escaping trajectories, in Fig. 30 we show the dependence of $r, \Omega$ and $\delta$ as a function of the average in- and out-couplings $K_{0}$ and $G_{0}$, considering oscillators with phase-coherent, $(a, b, c)=(0.15,0.2,10)$, and funnel attractors, $(a, b, c)=(0.2,0.2,7)$. Making use of the symmetry of the maps with respect to the main diagonal, the results concerning the phasecoherent case are depicted in the upper triangles, whereas results regarding oscillators with funnel attractors are shown in the lower one. For all simulations regarding system (5.51) the long-time behavior of these quantities is calculated by averaging data between $t \in[4700,5000]$ (see Appendix B). One notices the formation of a $\pi$-state in the region with $r \simeq 0$ [Fig. 3(a)] and $\delta \simeq \pi$ [Fig. 30(b)], which is surrounded by areas of partial synchronization with $0<\delta<\pi$ for both types of attractor, yet less prominent for funnel (values of $\delta$ were set to 0 in the incoherent region, since $\psi_{1}$ and $\psi_{2}$ are undefined in this state). Interestingly, in the dynamics of phase oscillators under attractive and repulsive interactions, one would expect the emergence of a collective rhythm different from the natural frequency in the lag-sync region (compare Figs. 24 and 30). Differently weighted $x$-coupled Rössler oscillators subjected to weak noise intensities present $\Omega \simeq \omega_{0}$ for $0 \leq$ $\delta \leq \pi$. On the other hand, for large negative values of $K_{0} G_{0}$, significant values of $\Omega \neq \omega_{0}$ 

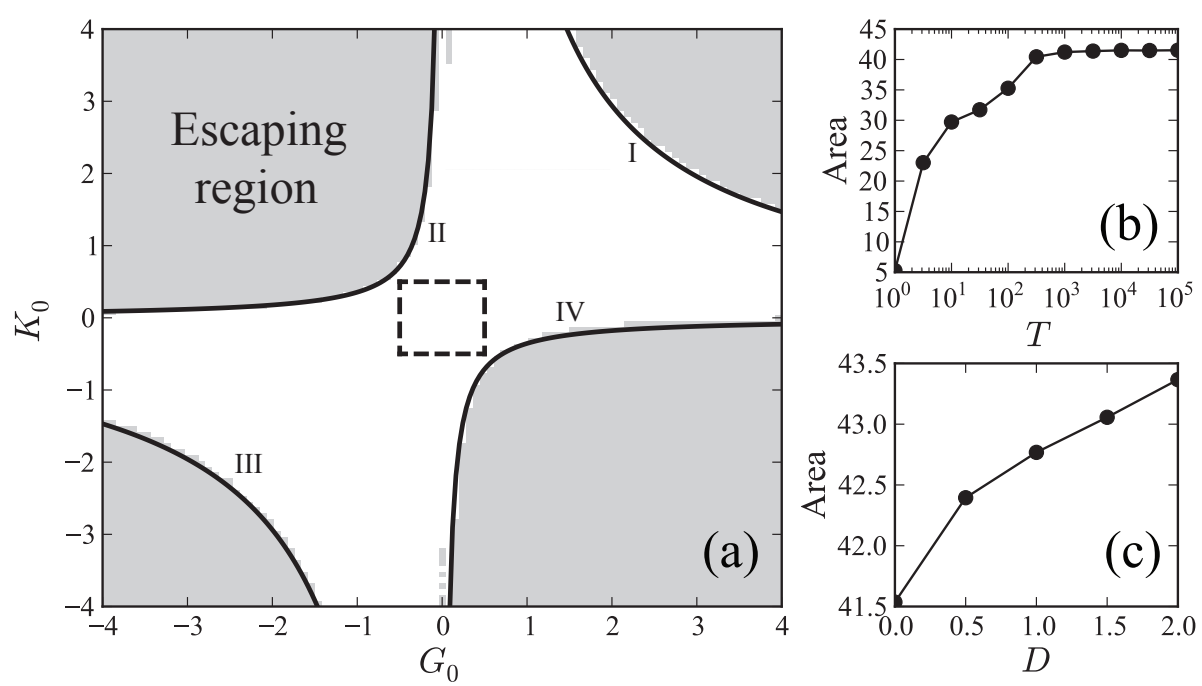

Figure 29 - (a) Map showing the regions with escaping trajectories for weighted $x$-coupled Rössler oscillators (Eq. 5.51) in the phase-coherent regime considering $N=500$, $D=0, \Delta K=0.1$, and $\Delta G=1$. For each pair $\left(G_{0}, K_{0}\right)$ the initial conditions $\left[x_{i}(0), y_{i}(0), z_{i}(0)\right] \forall i$ are randomly drawn according to a uniform distribution in the range $[-1,1]$. Simulation time $T=1 \times 10^{5}$. Solid black curves fitting the boundaries of the escaping region are obtained through the relation $\langle\langle K G\rangle\rangle=c$, where $c_{\mathrm{I}}=c_{\mathrm{III}}$ $=11.8$ and $c_{\mathrm{II}}=c_{\mathrm{IV}}=-0.66$. Square at the center depicts the parameter region of Fig. 30. Area of escaping region as function of (b) simulation time $T$ for fixed $D=0$ and (c) as function of $D$ for $T=5 \times 10^{3}$.

Source: Adapted from PERON et al. (161)

are observed in the incoherent region, as shown in Fig. 30(c). Correspondingly, we refer to this regime as incoherent traveling wave state.(161)

Comparing Fig. 29 and Fig. 30(c), we see that $\left|\Omega-\omega_{0}\right|$ increases as one approaches the boundaries of the escaping region, predicting in a way the occurrence of divergent trajectories. We stress though that this behavior is not a result due to fluctuations, but an emergent phenomenon that is yielded by the repulsive couplings considered in Eq. 5.51. Evidences of that can already be seen in Fig. 30. Note that deviations from the natural frequency $\omega_{0}$ are restricted to TW areas, while the $\pi$ and partially synchronized states are marked by an insignificant drift in the average frequency. Furthermore, as shown in Fig. 30(c), for the phase-coherent case, the drift in the frequency reaches a minimum before the wave sets in, a phenomenon not observed for oscillators with funnel attractors. In Appendix B we further show that in the system of Eq. 5.51, global and strong repulsive interactions without coupling mismatches suffice to generate incoherent traveling waves, even in populations with size up to $N=10^{6}$.

The influence of noise is shown in Fig. 30(d) considering phase-coherent oscillators. Of particular interest is the non-monotonic dependence on the noise strength by the $\pi$-state area. On the other hand, large noise completely extinguishes the traveling wave re- 

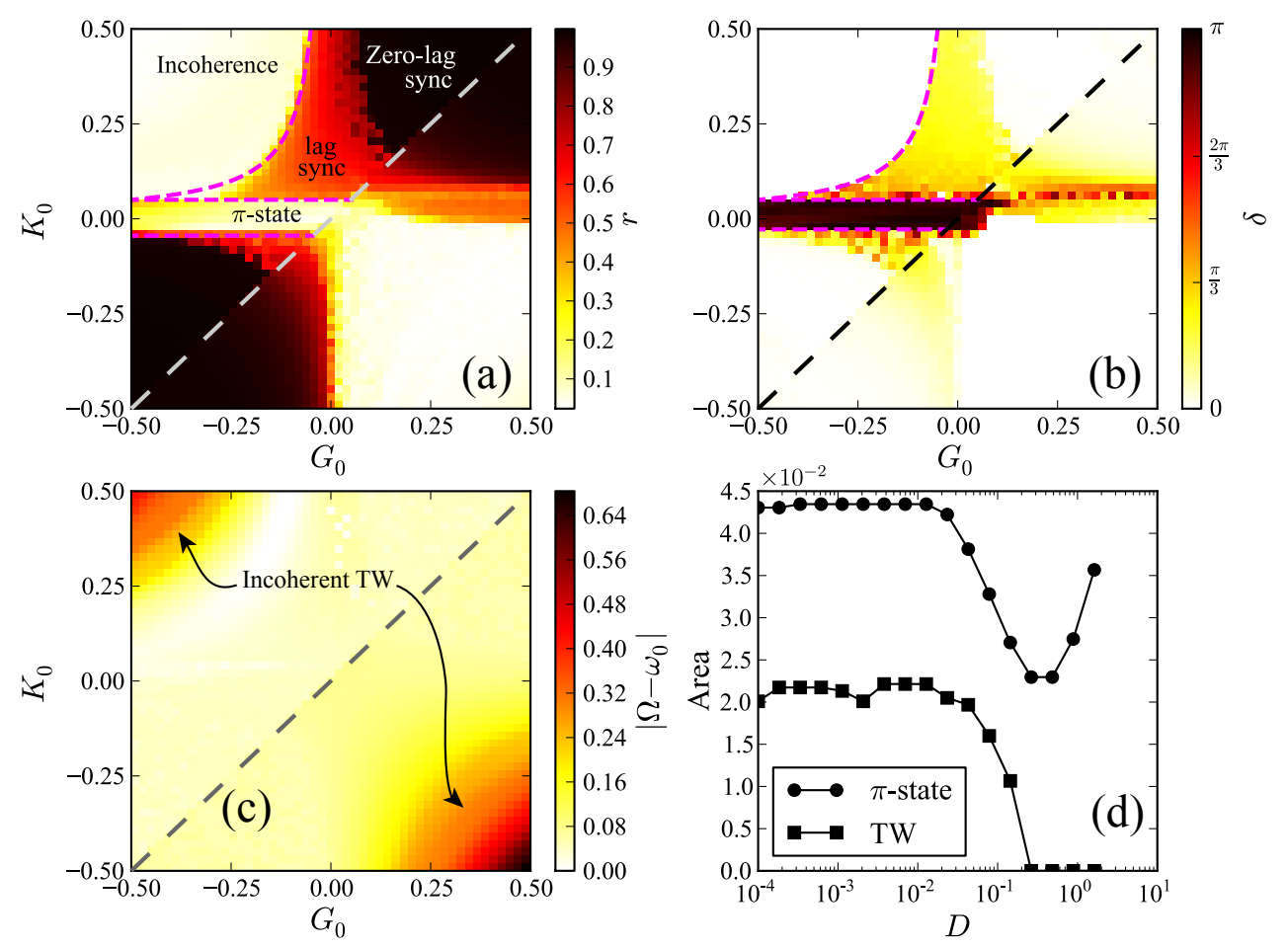

Figure 30 -Colormaps showing (a) order parameter $r$, (b) phase lag $\delta$ and (c) speed of phase wave $\left|\Omega-\omega_{0}\right|$ for weighted $x$-coupled Rössler oscillators (Eq. 5.51) with phase-coherent (upper triangles) and funnel (lower triangles) attractors considering $D=0.03$. (d) Area of TW state in which $\left|\Omega-\omega_{0}\right| \geq \varepsilon$, where we consider $\varepsilon=0.3$; and $\pi$-state for $\delta \geq \varepsilon$ with $\varepsilon=3$. Remaining parameters: $\Delta K=0.1$, $\Delta G=1, N=10^{3}, 50 \times 50$ grid, simulation time $T=5 \times 10^{3}$. Curves in the interface between incoherent and partial synchronized state for phase-coherent oscillators are given by the condition $2 D_{\text {eff }}=\langle\langle K G\rangle\rangle$, here with $D_{\text {eff }} \approx 0$. Horizontal dashed lines correspond to the critical points $K_{0 c}= \pm \Delta K / 2$. See also Appendix B for diagrams with $\Delta K=\Delta G=0$.

Source: Adapted from PERON et al. (161)

gions. Similar results are found for oscillators with funnel attractors (not shown). Despite the striking contrast between the nature of the traveling wave states observed in these systems, it is still possible to quantitatively describe certain aspects of the dynamics of chaotic oscillators with theories developed in the context of pure phase oscillators. Applying this condition in the weak noise case and considering the effective noise strength $D_{\text {eff }}{ }^{3}$, we see that the result uncovered for the Kuramoto model predicts with great accuracy the boundaries of incoherent states in networks of diffusively coupled Rössler oscillators. Conditions (5.37) are depicted by the horizontal lines in Figs. 30(a) and (b). As it is seen, the critical lines encompass the $\pi$-state area, similarly as in Fig. 24 for the Kuramoto model.

3 The effective noise strength is given by $D_{\text {eff }}=\frac{1}{2} \frac{d}{d t}\left[\left\langle\theta^{2}(t)\right\rangle-\langle\theta(t)\rangle^{2}\right] .(1$ 

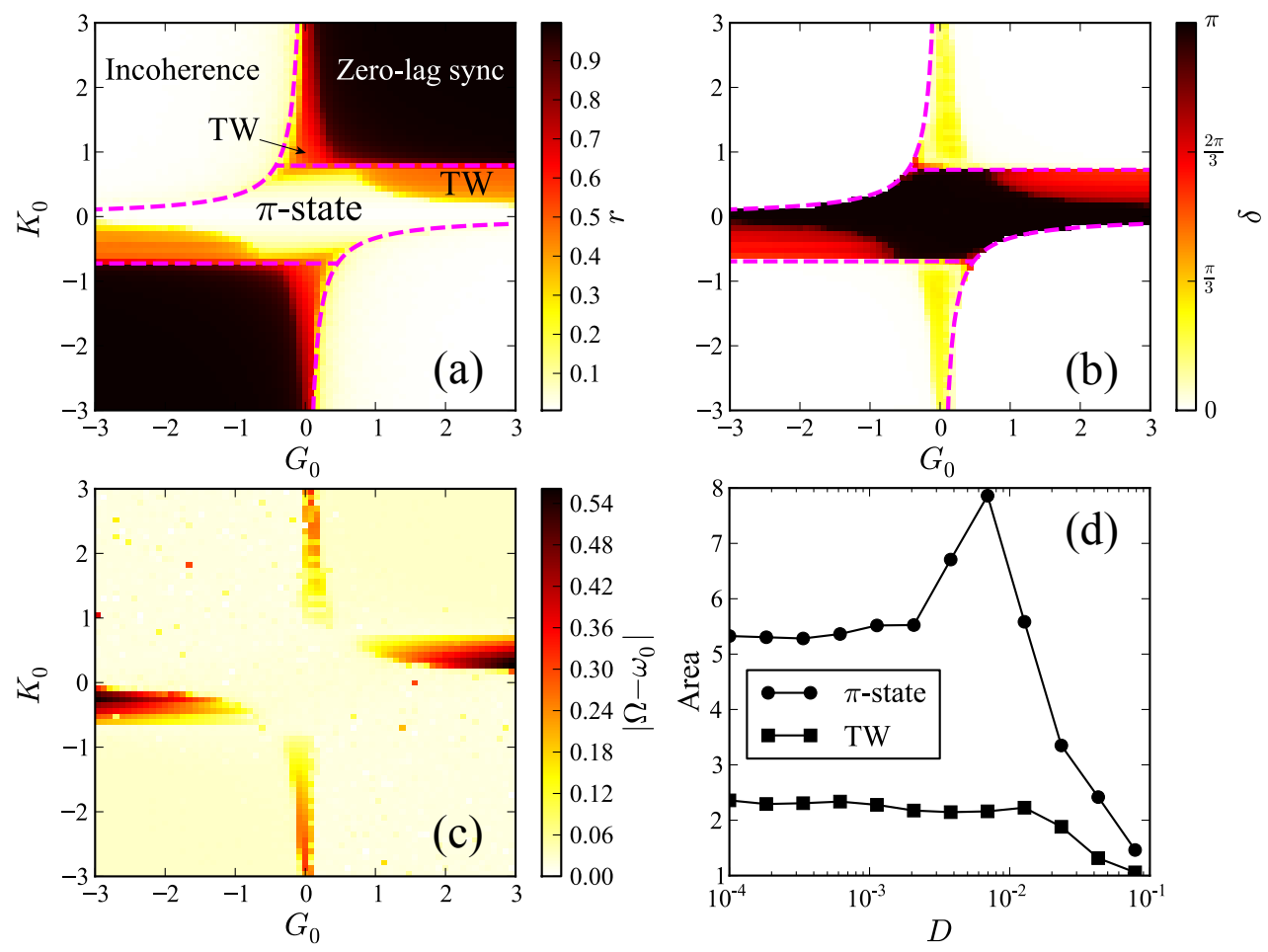

Figure 31 - Colormaps for the phase-coupled model (Eq. 5.57) showing (a) (total) order parameter $r$, (b) phase lag $\delta$, (c) the spontaneous drift measured by $\left|\Omega-\omega_{0}\right|$, where $\Omega$ is the wave speed (Eq. 5.55) and $\omega_{0}$ the natural frequency. (d) Area of TW state in which $\left|\Omega-\omega_{0}\right| \geq \varepsilon$, where we consider $\varepsilon=0.3$; and $\pi$-state for $\delta \geq \varepsilon$ with $\varepsilon=3$. For each point in the $70 \times 70$ grid, simulations are performed by evolving equations (5.57) considering initial conditions $\rho_{i}(0)$ and $z_{i}(0)$ randomly chosen from uniform distribution $[-1,1]$ and $\phi_{i}(0)$ uniformly distributed in the range $[-\pi, \pi]$. Other parameters: $N=10^{3}, \Delta K=1.45, \Delta G=1, D=0.03$, averaging over $t \in[4000,5000]$ and $d t=0.05$. Curves in the interface between incoherent and partial synchronized state are given by the condition $2 D=\langle\langle K G\rangle\rangle$. Horizontal dashed lines correspond to the critical points $K_{0 c}= \pm \Delta K / 2$. See Appendices B.4 and B.5 for additional diagrams of the phase-coupled model.

Source: Adapted from PERON et al. (161)

\subsubsection{Phase-coupled Rössler systems}

The levels of synchronization uncovered in the weighted $x$-coupled model share similarities with the ones found in ensembles of phase oscillators subjected to attractive and repulsive couplings of the previous sections. However, careful inspection of the collective frequency diagrams [Fig. 30(c)] reveals that the dynamical states in these systems are in fact different. Precisely, traveling waves among weighted $x$-coupled chaotic oscillators are manifested only in the absence of coherent oscillations, while partial synchronization is crucial for the emergence of such states in coupled phase oscillators (see Fig. 24). This poses the question of whether incoherent traveling waves are induced by the intrinsic chaotic dynamics or by the type of coupling adopted in Eq. 5.51. 
To shed light on this, we consider now a system of $N$ Rössler systems with $\mathrm{Ku}$ ramoto phase-couplings as considered in Sec. 5.1. In the phase-coherent regime it is valid to separate the original system into phase and amplitude dynamics. For this purpose, one goes to cylindrical coordinates $(\rho, \theta, z)$ via $x(t)=\rho(t) \cos \theta(t)$ and $y(t)=\rho(t) \sin \theta(t)$. This results in

$$
\begin{aligned}
& \dot{\theta}=\omega+a \sin \theta \cos \theta+\frac{z}{\rho} \sin \theta \\
& \dot{\rho}=a \rho \sin ^{2} \theta-z \cos \theta \\
& \dot{z}=b+\rho z \cos \theta-c z .
\end{aligned}
$$

We then formulate the phase-coupled model as (161)

$$
\begin{aligned}
& \dot{\theta}_{i}=\omega_{0}+a \sin \theta_{i} \cos \theta_{i}+\frac{z_{i}}{\rho_{i}} \sin \theta_{i}+\frac{K_{i}}{N} \sum_{j=1}^{N} G_{j} \sin \left(\theta_{j}-\theta_{i}\right)+\xi_{i}(t), \\
& \dot{\rho}_{i}=a \rho_{i} \sin ^{2} \theta_{i}-z_{i} \cos \theta_{i}, \\
& \dot{z}_{i}=b+\rho_{i} z_{i} \cos \theta_{i}-c z_{i} .
\end{aligned}
$$

We continue our analysis by getting a general view of the possible dynamical states of system Eqs. 5.57. In order to do so, Fig. 31 shows the simulation results of the total order parameter $r$, phase lag $\delta$ between the two populations, and the wave speed $\Omega$ which are now calculated with respect to the phases defined in Eq. 5.57. For each of the $70 \times 70$ points in the grid, system in Eq. 5.57 is numerically integrated by using the Heun's scheme with time step $d t=0.05$ and considering a population of $N=10^{3}$ oscillators for which the quantities of interest are averaged over $t \in[4000,5000]$. By inspecting panels (a)-(c) of Fig. 31, four well-defined states are uncovered, namely incoherent $\left(r_{1,2} \simeq 0, r \simeq 0\right.$ and $\left.\left|\Omega-\omega_{0}\right| \simeq 0\right)$, zero-lag sync $(r \simeq 1, \delta \simeq 0$ and $\Omega \simeq 0)$, $\pi$-state $(r \simeq 0, \delta \simeq \pi$ and $\left.\left|\Omega-\omega_{0}\right| \simeq 0\right)$ and traveling-wave $\left(r>0,0<\delta<\pi\right.$ and $\left.\Omega \neq \omega_{0}\right)$. Interestingly, in contrast to the weighted $x$-coupled model, traveling waves are not observed in the presence of incoherence if the oscillators are coupled only through their phase angles. Another difference between the coupling models is the fact that the areas of the $\pi$-state behave in a different manner as a function of the noise strength $D$ (compare panels $(\mathrm{d})$ of Fig. 30 and 31). Note that the critical condition $2 D=\langle\langle K G\rangle\rangle$ again predicts with high accuracy the boundaries of the incoherent state.

At last, let us give a brief overview on the effects of noise observed in the dynamics of coupled Rössler oscillators. (i) Noise enlarges the region of collective escape, but only in a surprisingly slight way, see Fig. 29(c). (ii) In the $x$-coupled case, the incoherent traveling waves disappear at a critical noise intensity, while the $\pi$-states are favored both by low and high noise intensities, as shown in Fig. 30(d). (iii) In the phase-coupled case, traveling wave states again disappear at large noise intensities, but $\pi$-states are most prominent at an intermediate noise intensity (see Fig. 31(d)). 


\subsection{Summary and outlook}

In this chapter we have slightly shifted the focus of our investigation to globally connected networks. Instead of posing the question of how the network structure shapes the dynamics, we asked: What other collective states can be observed if now the nature of the coupling between the oscillators is changed? In other words, is it possible for phase oscillators to manifest different dynamical states rather than incoherence and partial synchronization if the interactions are no longer purely attractive and symmetric as in standard formulations of the Kuramoto model? To tackle this issue we proposed a variation of the latter in which identical stochastic oscillators interact via in- and out-coupling strengths that were allowed to assume either positive and negative values. Heterogeneity in the interaction with the mean-field was introduced by separating the oscillators into two mutually coupled subpopulations, each one characterized by a distinct pair of couplings (cf. Fig. 20). By employing the Gaussian approximation technique, we were able to reduce the dimensionality of the original set of equations to a three-dimensional system of ODEs. The detailed bifurcation analysis of the reduced system allowed us to answer that yes, indeed other dynamical states take place if repulsive directed couplings are added to the model. We saw that these new self-organized patterns are characterized by the appearance of two in-phase clusters separated by a phase-lag whose value crucially impact on global oscillation rhythm. When the centroid of the phase distributions of each population are diametrically opposed, we obtain the so-called $\pi$-state. In such a configuration, the oscillators remain static in the co-rotating frame, i.e. the effective frequency at which the oscillators rotate coincides with the natural one. On the other hand, if the phase-lag between the population is less than $\pi$, the whole system agrees on a new rhythm - different from the intrinsinc one - and the traveling wave state sets in.

Comparing our results with previous fidings in the context of phase oscillators under the influence of attractive and repulsive interactions, we saw that the inclusion of in- and out-coupling strengths unfolded a whole new set of routes to the various collective states reported. More precisely, two paths towards traveling waves were uncovered, one via crossing $\pi$-state regions and the other by passing through areas of zero-lag sync. By analyzing the bifurcation diagrams in Fig. 22, we found that the latter route is only obtained if the oscillators interact under mixed out-couplings in the presence of non-mixed in-coupling strengths. This fact explains why traveling waves surrounded by partially synchronized states remained unnoticed in previous works.(156-158) Another surprising aspect observed in this chapter is the bistability found between all dynamical states for certain ranges of the parameters, highlighting a striking rich dynamical behavior given the simplicity of the phase oscillator model.

Inspired by the above findings, in the second part of this chapter, we asked whether 
mixed couplings are able to induce in chaotic systems analogous synchronization patterns as the ones reported for the Kuramoto model. To address this question, we analyzed Rössler oscillators in two distinct coupling scenarios. First, we connected the oscillators via a weighted linear coupling in the $x$ coordinates, and we considered thereby both chaotic regimes, the phase-coherent and the funnel attractor scenario. In both cases novel incoherent traveling waves were observed, which are characterized by the appearance of a new rhythm of oscillation without global synchronization. Secondly, we considered purely phase-coupled Rössler systems in the phase-coherent regime. In this second case, traveling waves were only found in conjunction with phase synchronization, namely in regions where the phase lag between the two synchronized groups fulfills $0<\delta<\pi$. These results on the one hand reinforce the potential that phase models possess in describing the dynamics of higher dimensional systems, but on the other hand they also highlight the limitations of phase models regarding other kinds of couplings, such as in the prediction of incoherent traveling waves. More precisely, in ensembles of phase oscillators and in phase-coupled Rössler systems introduced here, traveling waves are accessible if the subpopulations are separated by a phase lag whose existence is only possible under partial synchronization. However, the assumption that partial synchronization is necessary for the occurrence of traveling waves ceases to be true for diffusively coupled Rössler systems. Therefore, although the routes to phase synchronization of chaotic oscillators are qualitatively explained by phase models $(170,178)$, incoherent traveling waves cannot be described by such a decoupling of phases and amplitudes, since this phenomenon has no counterpart in ensembles of phase oscillators. This demonstrates a significant difference in the collective states observed in the phase dynamics of chaotic and phase oscillator systems.

Probably this chapter has raised more questions than it answers and, from the topics investigated here, we foresee many interesting new directions for future research. Regarding phase coupled oscillators, the first immeadiate continuation of our studies is to increase the level of complexity in the model by including heterogeneous connectitivity patterns. The first step towards this goal was already taken in Sec. 5.4, where we derived the reduced set of equations describing the collective dynamics of uncorrelated networks subjected to in- and out-coupling strengths. One promising line for future research is the determination of how the network topology alters the routes to the various states in the bifurcation diagrams of Fig. 22, or if topological features, such as degree-degree correlations, are able to favor or suppress a given dynamical state. Also in the matter of heterogeneous networks of attractive and repulsive oscillators, another topic deserves special interest. As mentioned before, one of the peculiarities that make the model so appealing is the existence of bistable areas that induce discontinuous jumps in the local and global parameters, as seen in Fig. 23. These results can potentially bring important new insights to investigations of the phenomenon of "explosive synchronization". In particular, 
great attention has been devoted to the determination of the mechanisms that trigger such a behavior in general complex networks.(32) Recently, it has been conjectured (179) that the necessary condition for the emergence of discontinuous transition is the existence of a suppressive rule that impedes the formation of a giant synchronous component. In this chapter, however, we showed - as an aside of our main results - that this conjecture may not be necessarily true. In fact, Fig. 23 provides a counterexample where we observe a discontinuous transition of the order parameters as function of the coupling strength in the absence of any suppressive rule that delays the emergence of a giant synchronous component. The resuls of Fig. 23 demonstrate instead that the discontinuity is a product of the bistability induced by the asymmetric interactions with the mean-field. It is important to remark though that our findings do not contradict previous results (179) concerning the emergence of explosive synchronization in the presence of rules that seek to undermine the formation of a synchronous component, but rather suggest that there could be different routes towards abrupt synchronization in networks.

Regarding coupled Rössler oscillators treated in Sec. 5.5.1, it remains as a future research to thoroughly determine the boundaries of the escaping region in Fig. 29 as well as the investigation of whether other chaotic systems also exhibit similar dynamical patterns as the ones described here. Likewise promising is the investigation of chimeralike states $(180,181)$ in the context of attractive and repulsive couplings. We further expect to observe the aforementioned states in real experiments with, for instance, electrochemical Belousov-Zhabotinsky oscillators.(182-184) 


\section{Conclusions}

The great goal of this thesis was to tackle fundamental issues regarding the dynamics of Kuramoto oscillators. The three major topics of our investigation were: (i) properly quantify the impact of clustering in network synchronization (Chapter 3), (ii) generalize the heterogeneous degree mean-field for networks of second-order oscillators (Chapter 4), and (iii) identify the role of asymmetric attractive and repulsive interactions between populations of identical oscillators (Chapter 5).

After briefly reviewing the evolution of network science and introducing the main concepts related to synchronization dynamics, we began Chapter 3 by discussing how the four classical network models are limited in reproducing topological features observed in real-world networks. We pointed out that, while each model is capable of individually capturing a specific characteristic - such as low shortest path lengths or heterogeneous degree distributions -, many others end up being overlooked. Among the properties left aside is the presence of triangles, subgraphs which can be seen as one of the simplest structural patterns that are pervasive in real systems but missing in random network models. It was then evident in our discussion that any attempt to thoroughly comprehend the dynamics of physical systems that exhibit realistic topologies is condemned to fail since an essential building block of their structure is in general neglected. We also saw that, in spite of the lack of a systematic way of controlling the occurrence of higher order features in network topology, alternative approaches considering stochastic rewiring algorithms have been employed in the literature in order to quantify how triangles affect the collective behavior of phase oscillators. The conclusions drawn in these studies suggested that clustering is pernicious for the emergence of coherent oscillations. However, as we discussed, the creation of triangles by these methods is always followed by a dramatic change of other network properties such as the formation of community structure and the appearance of degree-degree correlations. Thus, in this scenario, the sole impact of cycles of order three to the dynamics is potentially masked by side effects of the mechanism generating the networks. Our goal in Chapter 3 was to precisely elucidate this question, i.e. disentangle the influence of variations in the network transitivity from other metrics when assessing the collective behavior of Kuramoto oscillators. To this end, we considered the generalization of the configuration model by Newman and Miller which offers a practical way to vary the levels of transitivity while other metrics are kept constant. By adapting the heterogeneous degree mean-field we found that, surprisingly, the increasing of the number of triangles weakly affected the synchronization threshold of the networks. In other words, it was shown that one can, in fact, describe the phase dynamics of networks in terms of mean-field calculations conceived for locally tree-like networks even in 
the presence of significant values of clustering. This "unreasonable effectiveness" of treebased theories - as coined by Melnik et al. (71) - has actually been reported in other contexts, such as epidemic and rumor spreading, and in the Ising model.(32, 71, 93, 94) However, one needs to be careful and look at the limitations of the network model used before concluding on the innocuous effect of triadic connections. As argued in the end of Chapter 3, the networks generated by the Newman-Miller scheme are restricted to the low-clustering regime due to the fact that overlapping triangles are not contemplated in the construction process. While this leaves room for further studies considering models with more sophisticated topological structure, for now we can safely conclude that, at least in the low-clustering regime, clustered networks dynamically behave as unclustered ones. One could speculate that triangles start to play a role in network dynamics only when degree-degree correlations are no longer neglectable. In this case, it does not make sense to speak about the influence of one property disregarding contributions of the other since both are intrinsically related.(185) We therefore believe that future investigations on this matter should aim at developing field theories that simultaneously take into account different higher-order network properties.

The work in Chapter 3 was originated from our wish to understand real complex systems modeled by networks. However, the accurate description of their topology is only a part of the study; our motivation would remain vague if realistic dynamical aspects are not taken into account as well. This questioning brought us to Chapter 4 , where we have analyzed the second-order Kuramoto model, which turns out to reflect, to some extend, the global dynamics of power-grids. But instead of focusing on pratical applications to power systems, our main contribution from this chapter was to lay down the theoretical foundations of the first mean-field treatment of populations of second-order Kuramoto oscillators in uncorrelated networks. In fact, it appeared to us surprising that such a work had not been carried out many years before us given the popularity of network science and the growing interest in the modeling of power grids with the help of methods derived from statistical physics and dynamical systems theory.

The initial step of our endeavor was to adapt the approach by Tanaka et al. $(30,31)$ to heterogeneous topologies. Next, we applied our self-consistent analysis to the special case in which the natural frequencies depend on the degrees in scale-free networks. As expected from inertial phase oscillators, the system exhibited hysteretic synchronization transitions with abrupt variation of the order parameter, but with a particular difference from the first-order model under similar correlation between intrinsic dynamics and topology. More precisely, we verified that, despite the abrupt transition to the synchronous state, only a portion of the network becomes phase-locked, whereas in the first-order model (127) extreme values of the order parameter are achieved already at the critical coupling. Another interesting difference between the first- and second-order model is seen in the dependence of the transition on the amount of quenched disorder disturbing the 
natural frequencies. We saw that the greater the perturbation, the larger the hysteresis area, as it similarly occurs in the absence of inertia (144). However, the enhancement in the irreversible character of the order parameter comes along with the suppression of the discontinuity of the backward transition, in striking contrast with the results in (144). Finally, insights into practical domains can be gained from the investigation of secondorder Kuramoto oscillators in assortative networks. There, we revealed the interesting equivalence between dynamical and topological features with respect the stationary collective states achieved by the network. More specifically, we found that similar levels of synchronization can be obtained with different combinations of degree mixing and damping factors. This finding can be potentially relevant, for instance, in the design of power grids that are able to withstand structural failures more efficiently. In the face of a desynchronization threat, changes in the damping associated to certain power grid units could be performed in order to avoid massive outages. Such a strategy could be implemented in much more faster time scales than a reorganization of transmission lines which is a tremendous costly task. Interestingly, similar conclusions as ours were drawn in (27) via studying the stability conditions for the synchronous functioning of generators in realistic power grid networks. Overall, having set the scene for mean-field calculations of second-order Kuramoto oscillators in general uncorrelated networks, we believe the work in Chapter 4 may inspire further investigations on this topic in a similar way that the early works of Refs. $(70,73,186)$ helped to make the first-order Kuramoto model one of the most studied dynamical process in the complex network literature. $(32,69)$

In the last part of this thesis, instead of addressing the effects that the heterogeneity of network topology have on the synchronization dynamics, we focused on how phase oscillators behave if their interaction ceases to be symmetric and attractive. In other words, we were concerned with the question: what other collective states, other than the classical partially synchronized and incoherent, can take place if the nature of the network connections follows a different paradigm? By taking inspiration from other physical systems, we studied in Chapter 5 two intertwined populations of identical Kuramoto oscillators under the influence of asymmetric couplings which could either be negative or positive. Furthermore, a new ingredient in the model was also taken into account, namely sources of stochastic fluctuations in the phases' evolution which were modeled as white noise. At first glance, the inclusion of the latter feature may provoke the discomfort of probably increasing the complexity of the analysis; however, thanks to the inclusion of noise, we were able to employ the so-called Gaussian approximation technique whereby a dimensional reduction of the original system of equations was possible. More precisely, we showed that the original $N$ coupled equations of motion is reduced to a three-dimensional system of coupled ODE's, whose bifurcation diagram can be straightforwardly uncovered. By doing so, we then revealed two non-trivial arrangement of the oscillators - the so-called $\pi$ and traveling waves states. Both configurations are characterized by the existence of two 
partially synchronized clusters separated by a constant phase-lag. In the former, the two populations remain static and with the centroids anti-aligned in the space. However, if the phase-lag is less than $\pi$, the entire system agrees on a new rhythm of oscillation which is different from the natural frequency, defining then the traveling wave. Our bifurcation diagrams obtained through the reduced system revealed the existence of two routes to such states: one via crossing $\pi$-states and the other through classical partially synchronized states. We also showed that previous models reported in the literature can be obtained as particular cases from the coupling scheme of in- and out-coupling strengths proposed in this thesis, highlighting, despite its simplicity, the very rich dynamics of the model. Surprisingly, analogous collective states to the ones verified in the Kuramoto model were observed in ensembles of Rössler oscillators. In fact, not only a correspondence between the respective states were established, but also critical conditions obtained for pure phase oscillators were shown to predict boundaries in the parameter space of chaotic systems.

Something that contrasts Chapter 5 from the others is that its core is dedicated to the analysis of the dynamics on fully connected topologies. This seems to contradict the motto of this thesis that is to primarily focus on heterogeneous networks. However, such an approach was made necessary due to the novel character of the coupling scheme proposed. Essentially, we took the safest path of first isolating the effects of asymmetric attractive-repulsive couplings, permitting, in this way, the extension of the theory to general uncorrelated networks in Sec. 5.4. On the contrary, it would be troublesome to distinguish the influences of mixed couplings from the network topology itself. In fact, as it was clear in Sec. 5.4, the mere inclusion of what turns out to be the simplest heterogeneous topology (random binary networks) yields a bistable region in which two distinct partially synchronized states coexist - a phenomenon that remained unseen until the present work. Since this thesis needs to stop somewhere, we leave to further studies the thorough analysis of this peculiar behavior.

Suggestions for future research related to particular aspects of the findings reported here have already been given in the concluding parts of each chapter. Certainly, potential new explorations go far beyong the topics of this thesis. The Kuramoto model on complex networks has been a subject of intense scrutiny over the last two decades and yet there are still important issues to be tackled and other scenarios to be explored. The combination of different topologies and variations of the model dynamics are countless, leading us to believe that the years ahead are going to be as exciting as the last four.

$$
* * *
$$

The interplay between structure and dynamics is a fascinating problem that has driven a major part of the advances in network science. Examples of pertinent questions in the context of networked phase oscillators are: What are the most synchronizable net- 
works? And which are the ones that better sustain synchronization under perturbation? How does the order parameter scale with the system size? Does this scaling change with different choices of the degree distribution? What if the networks exhibit community structure or assortative properties? As we saw in this thesis, sometimes there is not a definite answer for questions of this nature and one should approach them carefully. On the one hand, this myriad of possible scenarios to be investigated creates an exciting atmosphere that certainly boosts the area by attracting more researchers and hence increasing its popularity. On the other hand, the many questions arising yield a propitious environment for the emergence of duplicated and even misleading reports, making it hard for these newcomers to be aware of the status quaestionis of a rapidly developing field. In other words, without a solid comprehension of the current state of the issues under study, new results cannot be properly discussed and, consequently, progress is difficult to be achieved. When we started this doctorate project almost five years ago, the collective dynamics of oscillatory networks was already a hot topic, with the first theoretical results on the Kuramoto model already featuring in classical texts of the complex network literature. $(20,39,69)$ However, since their publication the field has prospered greatly and, as in any popular area, one has witnessed the appearance of an overwhelming number of works addressing different facets of the model. Seeking to guide ourselves and to avoid misinterpreting certain results, we started a process of systematically organizing the contributions regarding the Kuramoto model in complex networks published in the last fifteen years. The outcome of our efforts finally culminated in a review paper published in 2016.(32) We sincerely hope this work inspires new debates and helps to orientate anyone interest on the field. 



\section{Bibliography}

1 PIKOVSKY, A.; ROSENBLUM, M.; KURTHS, J. Synchronization: a universal concept in nonlinear sciences. Cambridge: Cambridge University Press, 2003. (Cambridge nonlinear science series, v. 12).

2 STROGATZ, S. Sync: the emerging science of spontaneous order. New York: Hyperion, 2003.

3 BUCK, J.; BUCK, E. Mechanism of rhythmic synchronous flashing of fireflies. Science, v. 159, n. 3821, p. 1319-1327, 1968.

4 WONG, R.; TRAUB, R.; MILES, R. Cellular basis of neuronal synchrony in epilepsy. Advances in Neurology, v. 44, p. 583-592, 1985.

5 MORMANN, F. et al. Epileptic seizures are preceded by a decrease in synchronization. Epilepsy Research, v. 53, n. 3, p. 173-185, 2003.

6 HAMMOND, C.; BERGMAN, H.; BROWN, P. Pathological synchronization in parkinson's disease: networks, models and treatments. Trends in Neurosciences, v. 30, n. 7, p. 357-364, 2007.

7 KAPITANIAK, M. et al. Synchronization of clocks. Physics Reports, v. 517, n. 1, p. $1-69,2012$.

8 RAMIREZ, J. P. et al. The sympathy of two pendulum clocks: beyond Huygens' observations. Scientific Reports, v. 6, 2016. doi: 10.1038/srep23580.

9 WIENER, N. Nonlinear problems in Random theory. Cambridge: MIT Press, 1958.

10 WIENER, N. Cybernetics. Cambridge: MIT Press, 1961.

11 STROGATZ, S. H. From Kuramoto to crawford: exploring the onset of synchronization in populations of coupled oscillators. Physica D, v. 143, n. 1, p. 1-20.

12 WINFREE, A. T. Biological rhythms and the behavior of populations of coupled oscillators. Journal of Theoretical Biology, v. 16, n. 1, p. 15-42, 1967.

13 WINFREE, A. T. The geometry of biological time. New York: Springer, 1980.

14 KURAMOTO, Y. Self-entrainment of a population of coupled non-linear oscillators. In: ARAKI, H. (Ed.). INTERNATIONAL SYMPOSIUM ON MATHEMATICAL PROBLEMS IN THEORETICAL PHYSICS, 1975, Kyoto. Proceedings... New York: Springer-Verlag, 1975.

15 KURAmOTO, Y. Chemical oscillations, waves, and turbulence. Berlin: Springer, 1984 .

16 ACEBRÓN, J. A. et al. The Kuramoto model: a simple paradigm for synchronization phenomena. Reviews of Moderns Physics, v. 77, n. 1, p. 137, 2005. 
17 WATTS, D. J.; STROGATZ, S. H. Collective dynamics of small-world networks. Nature, v. 393, n. 6684, p. 440-442, 1998.

18 NEWMAN, M. Networks: an introduction. Oxford: Oxford University Press, 2010.

19 ALBERT, R.; BARABÁSI, A.-L. Statistical mechanics of complex networks. Reviews of Modern Physics, v. 74, n. 1, p. 47, 2002.

20 BOCCALETTI, S. et al. Complex networks: structure and dynamics. Physics Reports, v. 424, n. 4, p. 175-308, 2006.

21 COSTA, L. d. F. et al. Characterization of complex networks: a survey of measurements. Advances in Physics, v. 56, n. 1, p. 167-242, 2007.

22 COSTA, L. d. F. et al. Analyzing and modeling real-world phenomena with complex networks: a survey of applications. Advances in Physics, v. 60, n. 3, p. 329-412, 2011.

23 DOROGOVTSEV, S. N.; GOLTSEV, A. V.; MENDES, J. F. Critical phenomena in complex networks. Reviews of Modern Physics, v. 80, n. 4, p. 1275, 2008.

24 DOROGOVTSEV, S. N. Lectures on complex networks. Oxford: Oxford University Press, 2010. (Oxford master series in physics, v. 24).

25 BORNHOLDT, S.; SCHUSTER, H. G. Handbook of graphs and networks: from the genome to the internet. Berlin: John Wiley \& Sons, 2003.

26 ROHDEN, M. et al. Self-organized synchronization in decentralized power grids. Physical Review Letters, v. 109, n. 6, p. 064101, 2012.

27 MOTTER, A. E. et al. Spontaneous synchrony in power-grid networks. Nature Physics, v. 9, n. 3, p. 191-197, 2013.

28 MENCK, P. J. et al. How dead ends undermine power grid stability. Nature Communications, v. 5, p. 3969, 2014.

29 DÖRFLER, F.; BULLO, F. Synchronization in complex networks of phase oscillators: a survey. Automatica, v. 50, n. 6, p. 1539-1564, 2014.

30 TANAKA, H.-A.; LICHTENBERG, A. J.; OISHI, S. First order phase transition resulting from finite inertia in coupled oscillator systems. Physical Review Letters, v. 78, n. 11, p. 2104-2107, 1997.

31 TANAKA, H.-A.; LICHTENBERG, A. J.; OISHI, S. Self-synchronization of coupled oscillators with hysteretic responses. Physica D, v. 100, n. 3, p. 279-300, 1997.

32 RODRIGUES, F. A. et al. The Kuramoto model in complex networks. Physics Reports, v. 610, p. 1-98, 2016.

33 JI, P. et al. Cluster explosive synchronization in complex networks. Physical Review Letters, v. 110, n. 21, p. 218701, 2013.

34 DAIDO, H. Quasientrainment and slow relaxation in a population of oscillators with random and frustrated interactions. Physical Review Letters, v. 68, n. 7, p. 1073, 1992. 
35 BÖRGERS, C.; KOPELL, N. Synchronization in networks of excitatory and inhibitory neurons with sparse, random connectivity. Neural Computation, v. 15, n. 3, p. 509-538, 2003.

36 BINMORE, K. The breakdown of social contracts. In: DURLAUF, S.; YOUNG, P. (Ed.). Social dynamics. Cambridge: MIT Press, 2001. p. 213-236.

37 LINDNER, B. et al. Effects of noise in excitable systems. Physics Reports, v. 392, n. 6 , p. $321-424,2004$.

38 SONNENSCHEIN, B.; SCHIMANSKY-GEIER, L. Approximate solution to the stochastic Kuramoto model. Physical Review E, v. 88, n. 5, p. 052111, 2013.

39 BARRAT, A.; BARTHELEMY, M.; VESPIGNANI, A. Dynamical processes on complex networks. Cambridge: Cambridge University Press, 2008.

40 ESTRADA, E. When local and global clustering of networks diverge. Linear Algebra and Its Applications, v. 488, p. 249-263, 2016.

41 NEWMAN, M. E. The structure of scientific collaboration networks. Proceedings of the National Academy of Sciences, v. 98, n. 2, p. 404-409, 2001.

42 BOGUNA, M.; PASTOR-SATORRAS, R.; VESPIGNANI, A. Epidemic spreading in complex networks with degree correlations. 2003. Available at: $<$ https://arxiv.org/pdf/cond-mat/0301149.pdf>. Accessed in June 21, 2017.

43 NEWMAN, M. E. J. The structure and function of complex networks. SIAM Review, v. 45, n. 2, p. 167-256, 2003.

44 ERDÖS, P.; RÉNYI, A. On random graphs, I. Publicationes Mathematicae (Debrecen), v. 6, p. 290-297, 1959.

45 ERDOS, P.; RÉNYI, A. On the evolution of random graphs. Publication of the Mathematical Institute of the Hungarian Academy of Sciences, v. 5, n. 1, p. 17-60, 1960.

46 ERDOS, P.; RÉNYI, A. On the evolution of random graphs. Bulletin de l'Institut International de Statistique, v. 38, n. 4, p. 343-347, 1961.

47 ERDÖS, P.; RÉNYI, A. On the strength of connectedness of a random graph. Acta Mathematica Hungarica, v. 12, n. 1-2, p. 261-267, 1961.

48 SOLOMONOFF, R.; RAPOPORT, A. Connectivity of random nets. Bulletin of Mathematical Biology, v. 13, n. 2, p. 107-117, 1951.

49 GILBERT, E. N. Enumeration of labelled graphs. Canadian Journal of Mathematics, v. 8, n. 1, p. 05-411, 1956.

50 FORD, G.; UHLENBECK, G. Combinatorial problems in the theory of graphs. I. Proceedings of the National Academy of Sciences, v. 42, n. 3, p. 122-128, 1956.

51 AUSTIN, T. et al. The number of components in random linear graphs. Annals of Mathematical Statistics, v. 30, n. 3, p. 747-754, 1959.

52 BOLlOBÁS, B. Random graphs. Cambridge: Cambridge University Press, 2001. 
53 TRAVERS, J.; MILGRAM, S. An experimental study of the small world problem. Sociometry, v. 32, n. 4, p. 425-443, 1969.

54 REDNER, S. How popular is your paper? An empirical study of the citation distribution. European Physical Journal B, v. 4, n. 2, p. 131-134, 1998.

55 ALBERT, R.; JEONG, H.; BARABÁSI, A.-L. Internet: diameter of the world-wide web. Nature, v. 401, n. 6749, p. 130-131, 1999.

56 AMARAL, L. A. N. et al. Classes of small-world networks. Proceedings of the National Academy of Sciences, v. 97, n. 21, p. 11149-11152, 2000.

57 BARABÁSI, A.-L.; ALBERT, R. Emergence of scaling in random networks. Science, v. 286, n. 5439, p. 509-512, 1999.

58 MOLLOY, M.; REED, B. A critical point for random graphs with a given degree sequence. Random Structures 85 Algorithms, v. 6, n. 2-3, p. 161-180, 1995.

59 MILO, R. et al. Network motifs: simple building blocks of complex networks. Science, v. 298, n. 5594, p. 824-827, 2002.

60 NEWMAN, M. E. Random graphs with clustering. Physical Review Letters, v. 103, n. 5, p. 058701, 2009.

61 STROGATZ, S. H. From Kuramoto to Crawford: exploring the onset of synchronization in populations of coupled oscillators. Physica D, v. 143, n. 1, p. 1-20, 2000 .

62 Mirollo, R. E.; STROGATZ, S. H. The spectrum of the locked state for the Kuramoto model of coupled oscillators. Physica D, v. 205, n. 1, p. 249-266, 2005.

63 MIROLLO, R.; STROGATZ, S. H. The spectrum of the partially locked state for the Kuramoto model. Journal of Nonlinear Science, v. 17, n. 4, p. 309-347, 2007.

64 OTT, E.; ANTONSEN, T. M. Low dimensional behavior of large systems of globally coupled oscillators. Chaos, v. 18, n. 3, p. 037113, 2008.

65 LEE, T. E. et al. Vortices and the entrainment transition in the two-dimensional Kuramoto model. Physical Review E, v. 82, n. 3, p. 036202, 2010.

66 CHIBA, H. A proof of the Kuramoto conjecture for a bifurcation structure of the infinite-dimensional Kuramoto model. Ergodic Theory and Dynamical Systems, v. 35, n. 03, p. 762-834, 2015.

67 DIETERT, H. Stability and bifurcation for the Kuramoto model. Journal de Mathématiques Pures et Appliquées, v. 105, n. 4, p. 451-489, 2016.

68 FERNANDEZ, B.; GÉRARD-VARET, D.; GIACOMIN, G. Landau damping in the Kuramoto model. Annales Henri Poincaré, v. 17, n. 7, p. 1793-1823, 2016.

69 ARENAS, A. et al. Synchronization in complex networks. Physics Reports, v. 469, n. 3, p. 93-153, 2008.

70 RESTREPO, J. G.; OTT, E.; HUNT, B. R. Onset of synchronization in large networks of coupled oscillators. Physical Review E, v. 71, n. 3, p. 036151, 2005. 
71 MELNIK, S. et al. The unreasonable effectiveness of tree-based theory for networks with clustering. Physical Review E, v. 83, n. 3, p. 036112, 2011.

72 GLEESON, J. P. et al. Accuracy of mean-field theory for dynamics on real-world networks. Physical Review E, v. 85, n. 2, p. 026106, 2012.

73 ICHINOMIYA, T. Frequency synchronization in a random oscillator network. Physical Review E, v. 70, n. 2, p. 026116, 2004.

74 PASTOR-SATORRAS, R.; VESPIGNANI, A. Epidemic spreading in scale-free networks. Physical Review Letters, v. 86, n. 14, p. 3200, 2001.

75 PASTOR-SATORRAS, R. et al. Epidemic processes in complex networks. Reviews of Moderns Physics, v. 87, n. 3, p. 925, 2015.

76 MORENO, Y.; PACHECO, A. F. Synchronization of Kuramoto oscillators in scale-free networks. Europhysics Letters, v. 68, n. 4, p. 603, 2004.

77 KURAMOTO, Y. Kuramoto talks about the Kuramoto model. Available at: <https://youtu.be/lac4TxWyBOg>. Accessed in June 19, 2017.

78 HANSON, F. E. Comparative studies of firefly pacemakers. Federation Proceedings, v. 37 , n. 8, p. 2158-2164, 1978.

79 ERMENTROUT, B. An adaptive model for synchrony in the firefly Pteroptyx malaccae. Journal of Mathematical Biology, v. 29, n. 6, p. 571-585, 1991.

80 BUCK, J. Synchronous rhythmic flashing of fireflies. II. Quarterly Review of Biology, v. 63, n. 3, p. 265-289, 1988.

81 STROGATZ, S. H. Nonlinear dynamics and chaos: with applications to physics, biology, chemistry, and engineering. Cambridge: Westview Press, 2001.

82 GUCKENHEIMER, J.; HOLMES, P. Nonlinear oscillations, dynamical systems and bifurcations of vector fields. New York: Springer-Verlag, 1983.

83 MILLER, J. C. Percolation and epidemics in random clustered networks. Physical Review E, v. 80, n. 2, p. 020901, 2009.

84 PERON, T. K. D.; RODRIGUES, F. A.; KURTHS, J. Synchronization in clustered random networks. Physical Review E, v. 87, n. 3, p. 032807, 2013.

85 MCGRAW, P. N.; MENZINGER, M. Clustering and the synchronization of oscillator networks. Physical Review E, v. 72, n. 1, p. 015101, 2005.

86 MCGRAW, P. N.; MENZINGER, M. Analysis of nonlinear synchronization dynamics of oscillator networks by laplacian spectral methods. Physical Review E, v. 75, n. 2, p. 027104, 2007.

87 MCGRAW, P. N.; MENZINGER, M. Laplacian spectra as a diagnostic tool for network structure and dynamics. Physical Review E, v. 77, n. 3, p. 031102, 2008.

88 KIM, B. J. Performance of networks of artificial neurons: the role of clustering. Physical Review E, v. 69, n. 4, p. 045101, 2004. 
89 GÓMEZ-GARDENES, J.; MORENO, Y. Synchronization of networks with variable local properties. International Journal of Bifurcation and Chaos, v. 17, n. 07, p. 2501-2507, 2007.

90 XULVI-BRUNET, R.; SOKOLOV, I. Reshuffling scale-free networks: From random to assortative. Physical Review E, v. 70, n. 6, p. 066102, 2004.

91 ARRUDA, G. F. de et al. The influence of network properties on the synchronization of Kuramoto oscillators quantified by a bayesian regression analysis. Journal of Statistical Physics, v. 152, n. 3, p. 519-533, 2013.

92 HACKETT, A.; MELNIK, S.; GLEESON, J. P. Cascades on a class of clustered random networks. Physical Review E, v. 83, n. 5, p. 056107, 2011.

93 YOON, S. et al. Belief-propagation algorithm and the ising model on networks with arbitrary distributions of motifs. Physical Review E, v. 84, n. 4, p. 041144, 2011.

94 HERRERO, C. P. Ising model in clustered scale-free networks. Physical Review E, v. 91, n. 5, p. 052812, 2015.

95 SERRANO, M. Á.; BOGUNA, M. Clustering in complex networks. I. general formalism. Physical Review E, v. 74, n. 5, p. 056114, 2006.

96 SERRANO, M. Á.; BOGUNÁ, M. Clustering in complex networks. II. percolation properties. Physical Review E, v. 74, n. 5, p. 056115, 2006.

97 HUANG, X. et al. The robustness of interdependent clustered networks. Europhysics Letters, v. 101, n. 1, p. 18002, 2013.

98 HOLME, P.; KIM, B. J. Growing scale-free networks with tunable clustering. Physical Review E, v. 65, n. 2, p. 026107, 2002.

99 KLEMM, K.; EGUILUZ, V. M. Highly clustered scale-free networks. Physical Review E, v. 65, n. 3, p. 036123, 2002.

100 SERRANO, M. A.; BOGUNÁ, M. Tuning clustering in random networks with arbitrary degree distributions. Physical Review E, v. 72, n. 3, p. 036133, 2005.

101 BANSAL, S.; KHANDELWAL, S.; MEYERS, L. A. Exploring biological network structure with clustered random networks. BMC Bioinformatics, v. 10, n. 1, p. 405, 2009.

102 SHI, X.; ADAMIC, L. A.; STRAUSS, M. J. Networks of strong ties. Physica A, v. 378, n. 1, p. 33-47, 2007.

103 GLEESON, J. P. Bond percolation on a class of clustered random networks. Physical Review E, v. 80, n. 3, p. 036107, 2009.

104 KARRER, B.; NEWMAN, M. E. Random graphs containing arbitrary distributions of subgraphs. Physical Review E, v. 82, n. 6, p. 066118, 2010.

105 ZLATIĆ, V.; GARLASCHELLI, D.; CALDARELLI, G. Networks with arbitrary edge multiplicities. Europhysics Letters, v. 97, n. 2, p. 28005, 2012.

106 ALLARD, A. et al. Bond percolation on a class of correlated and clustered random graphs. Journal of Physics A, v. 45, n. 40, p. 405005, 2012. 
107 ALLARD, A. et al. Heterogeneous bond percolation on multitype networks with an application to epidemic dynamics. Physical Review E, v. 79, n. 3, p. 036113, 2009.

108 BERCHENKO, Y. et al. Emergence and size of the giant component in clustered random graphs with a given degree distribution. Physical Review Letters, v. 102, n. 13, p. 138701, 2009.

109 GHOSHAL, G. et al. Random hypergraphs and their applications. Physical Review E, v. 79, n. 6, p. 066118, 2009.

110 HÉBERT-DUFRESNE, L. et al. Percolation on random networks with arbitrary k-core structure. Physical Review E, v. 88, n. 6, p. 062820, 2013.

111 NEWMAN, M. E. J. Properties of highly clustered networks. Physical Review E, v. 68, n. 2, p. 026121, 2003.

112 SERRANO, M. A.; NÁ, M. B. Percolation and epidemic thresholds in clustered networks. Physical Review Letters, v. 97, n. 8, p. 088701, 2006.

113 VAZQUEZ, A. Spreading dynamics on heterogeneous populations: Multitype network approach. Physical Review E, v. 74, n. 6, p. 066114, 2006.

114 VÁZQUEZ, A.; MORENO, Y. Resilience to damage of graphs with degree correlations. Physical Review E, v. 67, n. 1, p. 015101, 2003.

115 SENDIÑA-NADAL, I. et al. Assortativity and leadership emerge from antipreferential attachment in heterogeneous networks. Scientific Reports, v. 6, 2016. doi: $10.1038 /$ srep21297.

116 RITCHIE, M.; BERTHOUZE, L.; KISS, I. Z. Generation and analysis of networks with a prescribed degree sequence and subgraph family: higher-order structure matters. Journal of Complex Networks, v. 5, n. 1, p. 1-31, 2017.

117 UNION FOR THE COORDINATION OF TRANSMISSION OF ELECTRICITY IN EUROPE. Final report-system disturbance on 4 november 2006. Technical report. Available from: <http://ecolo.org/documents/documents_in_english/ blackout-nov-06-UCTE-report.pdf>. Accessible at: 23 Mar. 2017.

118 KUndur, P.; BALU, N. J.; LAUBY, M. G. Power system stability and control. New York: McGraw-hill, 1994. (The EPRI power system engineering series, v.7).

119 MACHOWSKI, J.; BIALEK, J.; BUMBY, J. Power system dynamics: stability and control. Chichester: John Wiley \& Sons, 2011.

120 FIlATREllA, G.; NIELSEN, A. H.; PEDERSEN, N. F. Analysis of a power grid using a Kuramoto-like model. European Physical Journal B, v. 61, n. 4, 2008.

121 HILL, D. J.; CHEN, G. Power systems as dynamic networks. In: IEEE INTERNATIONAL SYMPOSIUM ON CIRCUITS AND SYSTEMS, 2006, Kos, Greece. Proceedings... New York: IEEE, 2006.

122 NISHIKAWA, T.; MOTTER, A. E. Comparative analysis of existing models for power-grid synchronization. New Journal of Physics, v. 17, n. 1, p. 015012, 2015. 
123 JI, P. et al. Cluster explosive synchronization in complex networks. Physical Review Letters, v. 110, n. 21, p. 218701. doi: 10.1103/PhysRevLett.110.218701.

124 JI, P. et al. Analysis of cluster explosive synchronization in complex networks. Physical Review E, v. 90, n. 6, p. 062810.

125 JI, P. et al. Low-dimensional behavior of Kuramoto model with inertia in complex networks. Scientific Reports, v. 4, p. 4783, 2014. doi: doi:10.1038/srep04783.

126 PERON, T. K. D. et al. Effects of assortative mixing in the second-order Kuramoto model. Physical Review E, v. 91, n. 5, p. 052805, 2015.

127 GÓMEZ-GARDEÑES, J. et al. Explosive synchronization transitions in scale-free networks. Physical Review Letters, v. 106, n. 12, p. 128701, 2011.

128 ZHU, L.; TIAN, L.; SHI, D. Criterion for the emergence of explosive synchronization transitions in networks of phase oscillators. Physical Review E, v. 88, n. 4, p. 042921. doi: 10.1103/PhysRevE.88.042921.

129 LI, M. et al. Effect of mixing parts of modular networks on explosive synchronization. Europhysics Letters, v. 104, n. 5, p. 58002, 2013.

130 LIU, W. et al. Effects of frequency-degree correlation on synchronization transition in scale-free networks. Europhysics Letters, v. 101, n. 3, p. 38002, 2013.

131 LI, P. et al. Reexamination of explosive synchronization in scale-free networks: The effect of disassortativity. Physical Review E, v. 87, n. 4, p. 042803, 2013.

132 SENDIÑA-NADAL, I. et al. Effects of degree correlations on the explosive synchronization of scale-free networks. Physical Review E, v. 91, n. 3, p. 032811, 2015.

133 SONNENSCHEIN, B.; SAGUÉS, F.; SCHIMANSKY-GEIER, L. Networks of noisy oscillators with correlated degree and frequency dispersion. European Physical Journal $B$, v. 86, n. 12 , p. 1-6, 2013.

134 PERON, T. K. D.; RODRIGUES, F. A. Determination of the critical coupling of explosive synchronization transitions in scale-free networks by mean-field approximations. Physical Review E, v. 86, n. 5, p. 056108, 2012.

135 PERON, T. K. D.; RODRIGUES, F. A. Explosive synchronization enhanced by time-delayed coupling. Physical Review E, v. 86, n. 1, p. 016102, 2012.

136 COUTINHO, B. et al. Kuramoto model with frequency-degree correlations on complex networks. Physical Review E, v. 87, n. 3, p. 032106, 2013.

137 SKARDAL, P. S. et al. Effects of degree-frequency correlations on network synchronization: Universality and full phase-locking. Europhysics Letters, v. 101, n. 2, p. 20001, 2013.

138 LEYVA, I. et al. Explosive synchronization in weighted complex networks. Physical Review E, v. 88, n. 4, p. 042808, 2013.

139 VARSHNEY, L. R. et al. Structural properties of the caenorhabditis elegans neuronal network. PLOS Computational Biology, v. 7, n. 2, p. e1001066, 2011. 
140 BREDE, M. Synchrony-optimized networks of non-identical Kuramoto oscillators. Physics Letters A, v. 372, n. 15, p. 2618-2622.

141 BUZNA, L.; LOZANO, S.; DÍAZ-GUILERA, A. Synchronization in symmetric bipolar population networks. Physical Review E, v. 80, n. 6, p. 066120, 2009.

142 SKARDAL, P. S.; TAYLOR, D.; SUN, J. Optimal synchronization of complex networks. Physical Review Letters, v. 113, n. 14, p. 144101, 2014.

143 OLMI, S. et al. Hysteretic transitions in the Kuramoto model with inertia. Physical Review E, v. 90, n. 4, p. 042905, 2014.

144 SKARDAL, P. S.; ARENAS, A. Disorder induces explosive synchronization. Physical Review E, v. 89, n. 6, p. 062811, 2014.

145 ROSAS-CASALS, M.; VALVERDE, S.; SOLÉ, R. V. Topological vulnerability of the european power grid under errors and attacks. International Journal of Bifurcation and Chaos, v. 17, n. 07, p. 2465-2475, 2007.

146 PAGANI, G. A.; AIELLO, M. The power grid as a complex network: a survey. Physica A, v. 392, n. 11, p. 2688-2700, 2013.

147 CHEN, H. et al. Explosive synchronization transitions in complex neural networks. Chaos, v. 23, n. 3, p. 033124, 2013.

148 NEWMAN, M. E. Assortative mixing in networks. Physical Review Letters, v. 89, n. 20, p. 208701, 2002.

149 GLEESON, J. P.; MELNIK, S.; HACKETT, A. How clustering affects the bond percolation threshold in complex networks. Physical Review E, v. 81, n. 6, p. 066114, 2010 .

150 ZHU, L.; TIAN, L.; SHI, D. Criterion for the emergence of explosive synchronization transitions in networks of phase oscillators. Physical Review E, v. 88, n. 4, p. 042921, 2013.

151 BARRÉ, J.; MÉTIVIER, D. Bifurcations and singularities for coupled oscillators with inertia and frustration. Physical Review Letters, v. 117, n. 21, p. 214102, 2016.

152 RESTREPO, J. G.; OTT, E. Mean-field theory of assortative networks of phase oscillators. Europhysics Letters, v. 107, n. 6, p. 60006, 2014.

153 SHERRINGTON, D.; KIRKPATRICK, S. Solvable model of a spin-glass. Physical Review Letters, v. 35, n. 26, p. 1792, 1975.

154 STILLER, J.; RADONS, G. Self-averaging of an order parameter in randomly coupled limit-cycle oscillators. Physical Review E, v. 61, n. 2, p. 2148, 2000.

155 DAIDO, H. Algebraic relaxation of an order parameter in randomly coupled limit-cycle oscillators. Physical Review E, v. 61, n. 2, p. 2145, 2000.

156 HONG, H.; STROGATZ, S. H. Kuramoto model of coupled oscillators with positive and negative coupling parameters: an example of conformist and contrarian oscillators. Physical Review Letters, v. 106, n. 5, p. 054102, 2011. 
157 HONG, H.; STROGATZ, S. H. Conformists and contrarians in a Kuramoto model with identical natural frequencies. Physical Review E, v. 84, n. 4, p. 046202, 2011.

158 HONG, H.; STROGATZ, S. H. Mean-field behavior in coupled oscillators with attractive and repulsive interactions. Physical Review E, v. 85, n. 5, p. 056210, 2012.

159 SONNENSCHEIN, B. Collective dynamics in complex networks of noisy phase oscillators: towards models of neuronal network dynamics. Berlin: Logos Verlag Berlin GmbH, 2016.

160 SONNENSCHEIN, B. et al. Collective dynamics in two populations of noisy oscillators with asymmetric interactions. Physical Review E, v. 91, n. 6, p. 062910, 2015.

161 PERON, T. K. D. et al. Traveling phase waves in asymmetric networks of noisy chaotic attractors. Physical Review E, v. 94, n. 4, p. 042210, 2016.

162 OTT, E.; ANTONSEN, T. M. Long time evolution of phase oscillator systems. Chaos, v. 19, n. 2, p. 023117, 2009.

163 SONNENSCHEIN, B. et al. Excitable elements controlled by noise and network structure. European Physical Journal Special Topics, v. 222, n. 10, p. 2517-2529, 2013.

164 SONNENSCHEIN, B. et al. Cooperative behavior between oscillatory and excitable units: the peculiar role of positive coupling-frequency correlations. European Physical Journal B, v. 87, n. 8, p. 1-11, 2014.

165 MARDIA, K. V.; JUPP, P. E. Directional statistics. Chichester: John Wiley \& Sons, 2009.

166 STROGATZ, S. H.; MIROLLO, R. E. Stability of incoherence in a population of coupled oscillators. Journal of Statistical Physics, v. 63, n. 3-4, p. 613-635, 1991.

167 SAKAGUCHI, H. Cooperative phenomena in coupled oscillator systems under external fields. Progress of Theoretical Physics, v. 79, n. 1, p. 39-46, 1988.

168 DHOOGE, A.; GOVAERTS, W.; KUZNETSOV, Y. A. Matcont: a matlab package for numerical bifurcation analysis of odes. ACM Transactions on Mathematical Software (TOMS), v. 29, n. 2, p. 141-164, 2003.

169 PETKOSKI, S. et al. Mean-field and mean-ensemble frequencies of a system of coupled oscillators. Physical Review E, v. 87, n. 3, p. 032908, 2013.

170 ROSEnBluM, M. G.; PIKOVSKY, A. S.; KURTHS, J. Phase synchronization of chaotic oscillators. Physical Review Letters, v. 76, n. 11, p. 1804, 1996.

171 ROSEnBluM, M. G.; PIKOVSKY, A. S.; KURTHS, J. From phase to lag synchronization in coupled chaotic oscillators. Physical Review Letters, v. 78, n. 22, p. 4193, 1997.

172 OSIPOV, G. V.; KURTHS, J.; ZHOU, C. Synchronization in oscillatory networks. Berlin: Springer, 2007.

173 RÖSSLER, O. E. An equation for continuous chaos. Physics Letters A, v. 57, n. 5, p. 397-398, 1976. 
174 GABOR, D. Theory of communication. Part 1: the analysis of information. Journal of the Institution of Electrical Engineers-Part III, v. 93, n. 26, p. 429-441, 1946.

175 FREUND, J. A.; SCHIMANSKY-GEIER, L.; HÄNGGI, P. Frequency and phase synchronization in stochastic systems. Chaos, v. 13, n. 1, p. 225-238, 2003.

176 LETELliER, C.; DUTERTRE, P.; MAHEU, B. Unstable periodic orbits and templates of the Rössler system: toward a systematic topological characterization. Chaos, v. 5, n. 1, p. 271-282, 1995.

177 BARRIO, R.; BLESA, F.; SERRANO, S. Unbounded dynamics in dissipative flows: Rössler model. Chaos, v. 24, n. 2, p. 024407, 2014.

178 ROSEnBlum, M. G.; PIKOVSKY, A. S.; KURTHS, J. From phase to lag synchronization in coupled chaotic oscillators. Physical Review Letters, v. 78, n. 22, p. 4193, 1997.

179 ZHANG, X. et al. Explosive synchronization in adaptive and multilayer networks. Physical Review Letters, v. 114, n. 3, p. 038701, 2015.

180 OMELCHENKO, I. et al. Loss of coherence in dynamical networks: spatial chaos and chimera states. Physical Review Letters, v. 106, n. 23, p. 234102, 2011.

181 MAISTRENKO, Y.; PENKOVSKY, B.; ROSENBLUM, M. Solitary state at the edge of synchrony in ensembles with attractive and repulsive interactions. Physical Review E, v. 89, n. 6, p. 060901, 2014.

182 KISS, I. Z. et al. Engineering complex dynamical structures: sequential patterns and desynchronization. Science, v. 316, n. 5833, p. 1886-1889, 2007.

183 TAYLOR, A. F. et al. Phase clusters in large populations of chemical oscillators. Angewandte Chemie, v. 123, n. 43, p. 10343-10346, 2011.

184 TOTZ, J. F. et al. Phase-lag synchronization in networks of coupled chemical oscillators. Physical Review E, v. 92, n. 2, p. 022819, 2015.

185 ESTRADA, E. Combinatorial study of degree assortativity in networks. Physical Review E, v. 84, n. 4, p. 047101, 2011.

186 LEE, D.-S. Synchronization transition in scale-free networks: clusters of synchrony. Physical Review E, v. 72, n. 2, p. 026208, 2005. 



\section{APPENDIX A - Contribution of drifting oscillators in the second-order Kuramoto model}

In this Appendix we shall estimate the contribution of drifting oscillators to the total order parameter in the second-order Kuramoto model. First we will present in detail the estimation for the model in the fully connected graph discussed in Sec. 2.2.2 and initially considered by Tanaka et al. $(30,31)$ In the sequence we will discuss how to generalize the procedure to the case treated in Chapter 4 of oscillators coupled in uncorrelated networks.

The contribution of the drifting oscillators derived in Sec. 2.2.2.1 is given by

$$
R_{\mathrm{drift}}^{\mathrm{I}, \mathrm{II}}=\frac{1}{\pi} \int_{|\omega|>\omega_{\mathrm{P}, \mathrm{D}}} \int_{0}^{\tilde{T}} \cos \theta(t, \omega)|\tilde{\omega}| g(\omega) d t d \omega .
$$

Thus, the whole problem relies on the estimation of the integral $\int_{0}^{\tilde{T}} \cos (t, \omega) d t$, where $\tilde{T}$ is the period of the running periodic solution of

$$
m \ddot{\theta}+\dot{\theta}+\lambda R \sin \theta=\omega .
$$

One possible way to proceed is to consider the underdamped case and introduce a small parameter $\varepsilon=(m \lambda)^{-1} \ll 1$. This motivates the following change of time scale $t \rightarrow \lambda^{-1} \tau$, which leads to

$$
\frac{d^{2} \theta}{d \tau^{2}}+\varepsilon \frac{d \theta}{d \tau}+\varepsilon^{2} \tilde{r} \sin \theta=\varepsilon \frac{\omega}{\lambda},
$$

where $R=\varepsilon \tilde{r}$, with $\tilde{r}$ being an arbitrary constant. At first, one could seek solutions of (A.2) in the form of a power series in $\varepsilon \theta(\tau)=\theta_{0}(\tau)+\varepsilon \theta_{1}(\tau)+\varepsilon^{2} \theta_{2}(\tau)+\ldots$, and perform the integration $\int_{0}^{\tilde{T}} \cos (\tau) d \tau$ perturbatively. However, the application of the regular pertubation theory produces solutions for the unknown functions $\theta_{k}(\tau)$ that are proportional to secular terms of the form $\tau \sin \tau$ and $\tau \cos \tau$, i.e. terms that will grow without bounds as $\tau \rightarrow \infty$ and that definitely do not reflect the oscillatory behavior of system (A.2). The solution for this is to consider the improved version of the standard perturbation analysis known as the Poincaré-Lindstead method.(81) The method consists in setting a new time scale

$$
s=\tilde{\omega}(\varepsilon) \tau
$$

where we also assume a perturbation expansion of $\tilde{\omega}(\varepsilon)$ as

$$
\tilde{\omega}(\varepsilon)=\omega_{0}+\varepsilon \omega_{1}+\varepsilon^{2} \omega_{2}+\cdots .
$$


In the new time scale $s$, Eq. A.2 reads

$$
\tilde{\omega}^{2}(\varepsilon) \frac{d^{2} \theta}{d s^{2}}+\varepsilon \tilde{\omega}(\varepsilon) \frac{d \theta}{d s}+\varepsilon^{2} \tilde{r} \sin \theta(\varepsilon)=\varepsilon \frac{\omega}{\lambda} .
$$

Next, we seek a perturbation expansion of $\theta(s)$ as

$$
\theta(s, \varepsilon)=\theta_{0}(s)+\varepsilon \theta_{1}(s)+\varepsilon^{2} \theta_{2}(s)+\cdots
$$

whose period is $\tilde{T}(\varepsilon)=2 \pi / \tilde{\omega}(\varepsilon)$. Substituting Eqs. A.4 and A.6 in Eq. A.5 we obtain

$$
\begin{array}{r}
\left(\omega_{0}+\varepsilon \omega_{1}+\varepsilon^{2} \omega_{2}+\varepsilon^{3} \omega_{3}+\cdots\right)^{2} \times\left(\theta_{0}^{\prime \prime}+\varepsilon \theta_{1}^{\prime \prime}+\varepsilon^{2} \theta_{2}^{\prime \prime}+\varepsilon^{3} \theta_{3}^{\prime \prime}+\cdots\right) \\
+\left(\varepsilon \omega_{0}+\varepsilon^{2} \omega_{1}+\varepsilon^{3} \omega_{2}+\varepsilon^{4} \omega_{3}+\cdots\right) \times\left(\theta_{0}^{\prime}+\varepsilon \theta_{1}^{\prime}+\varepsilon^{2} \theta_{2}^{\prime}+\varepsilon^{3} \theta_{3}^{\prime}+\cdots\right) \\
+\tilde{r}\left[\varepsilon^{2} \sin \theta_{0}+\left(\varepsilon^{3} \theta_{1}+\varepsilon^{4} \theta_{2}+\varepsilon^{5} \theta_{3}+\cdots\right) \cos \theta_{0}\right]=\varepsilon \frac{\omega}{\lambda}
\end{array}
$$

where the primes denote the differentiation with respect to variable $s$. Note also that the term $\sin \left[\theta_{0}(s)+\varepsilon \theta_{1}(s)+\varepsilon^{2} \theta_{2}(s)+\cdots\right]$ was expanded around $\theta_{0}(s)$. The task left is to determine the functions $\theta_{k}(s)$ and the terms $\omega_{k}$ by matching the coefficients at each power of $\varepsilon$.

Let the initial conditions be $\theta_{0}(0)=1$ and $\theta_{k}(0)=0$ for $k>0$. For order $\mathcal{O}(1)$ we have that

$$
\mathcal{O}(1): \omega_{0}^{2} \theta_{0}^{\prime \prime}=0 \Rightarrow \theta_{0}(s)=s
$$

Next, the matching of coefficients with terms $\varepsilon$ yields

$$
\begin{gathered}
\mathcal{O}(\varepsilon): \omega_{0}^{2} \theta_{1}^{\prime \prime}+2 \omega_{0} \omega_{1} \theta_{0}^{\prime \prime}+\omega_{0} \theta_{0}^{\prime}=0 \\
\theta_{1}^{\prime \prime}=\frac{1}{\omega_{0}^{2}}\left(\frac{\omega}{\lambda}-\omega_{0}\right) .
\end{gathered}
$$

Note that the above equation for $\theta_{1}^{\prime \prime}$ leads to an unbounded solution proportional to $s^{2}$, which eventually will become greater than the solution of $\theta_{0}(s)$. This is precisely the inconsistency we want to avoid and the only way of preventing it is by setting

$$
\omega_{0}=\frac{\omega}{\lambda}
$$

which together with the initial condition $\theta_{1}(0)=0$ gives us $\theta_{1}=0$. Continuing for higher order of $\varepsilon$ with this procedure of adjusting the terms $\omega_{k}$ so that potential divergent terms 
are eliminated leads to

$$
\begin{gathered}
\mathcal{O}\left(\varepsilon^{2}\right): \omega_{0}^{2} \theta_{2}^{\prime \prime}+2 \omega_{0} \omega_{1} \theta_{1}^{\prime \prime}+\omega_{1}^{2} \theta_{0}^{\prime}+2 \omega_{0} \omega_{2} \theta_{0}^{\prime \prime}+\omega_{0} \theta_{1}^{\prime}+\tilde{r} \sin \theta_{0}=0 \\
\omega_{0}^{2} \theta_{2}^{\prime \prime}+\omega_{1}^{2} \theta_{0}^{\prime}+\tilde{r} \sin \theta_{0}=0 \\
\Rightarrow \theta_{2}=\frac{\tilde{r}}{\omega_{0}^{2}} \sin \theta_{0}(s) \text { with } \omega_{1}=0 \\
\mathcal{O}\left(\varepsilon^{3}\right): \omega_{0}^{2} \theta_{3}^{\prime \prime}+2 \omega_{0} \omega_{2} \theta_{1}^{\prime \prime}+2 \omega_{0} \omega_{3} \theta_{0}^{\prime \prime}+\omega_{0} \theta_{2}^{\prime}+\omega_{2} \theta_{0}^{\prime}+\tilde{r} \theta_{1} \cos \theta_{1}=0 \\
\omega_{0} \theta_{3}^{\prime \prime}+\omega_{0} \theta_{2}^{\prime}+\omega_{2}+\tilde{r} \theta_{1} \cos \theta_{0}=0 \\
\Rightarrow \theta_{3}=\frac{\tilde{r}}{\omega_{0}^{3}}\left(\cos \theta_{0}-1\right) \text { and } \omega_{2}=0 \\
\mathcal{O}\left(\varepsilon^{4}\right): \omega_{0}^{2} \theta_{4}^{\prime \prime}+\omega_{0} \theta_{3}^{\prime}+\omega_{3} \theta_{0}^{\prime}+\tilde{r} \theta_{2} \cos \theta_{0}=0 \\
\omega_{0} \theta_{4}^{\prime \prime}-\frac{\tilde{r}}{\omega_{0}^{2}} \sin \theta_{0}+\frac{\tilde{r}^{2}}{\omega_{0}^{2}} \sin \theta_{0} \cos \theta_{0}+\omega_{3}=0 \\
\Rightarrow \theta_{4}=-\frac{\tilde{r}}{\omega_{0}^{4}} \sin \theta_{0}+\frac{1}{8} \frac{\tilde{r}^{2}}{\omega_{0}^{4}} \sin 2 \theta_{0} \text { and } \omega_{3}=0
\end{gathered}
$$

Finally, for order $\varepsilon^{5}$ :

$$
\begin{aligned}
\mathcal{O}\left(\varepsilon^{5}\right): & \omega_{0}^{2} \theta_{5}^{\prime \prime}+\omega_{0} \theta_{4}^{\prime}+\theta_{0}^{\prime} \omega_{4}+\tilde{r} \theta_{3} \cos \theta_{0}=0 \\
& \omega_{0}^{2} \theta_{5}^{\prime \prime}-\frac{\left(\tilde{r}+\tilde{r}^{2}\right)}{\omega_{0}^{3}} \cos \theta_{0}+\frac{\tilde{r}^{2}}{\omega_{0}^{3}}\left[\frac{1}{4} \cos 2 \theta_{0}+\cos ^{2} \theta_{0}\right]+\omega_{4}=0
\end{aligned}
$$

which integrated in $s$ yields

$$
\omega_{0}^{2} \theta_{5}^{\prime}-\frac{\left(\tilde{r}+\tilde{r}^{2}\right)}{\omega_{0}^{3}} \sin \theta_{0}+\frac{3}{4} \frac{\tilde{r}^{2}}{\omega_{0}^{3}} \cos 2 \theta_{0}+\underbrace{\frac{1}{2} \frac{\tilde{r}^{2}}{\omega_{0}^{3}} \theta_{0}+\omega_{4} s}_{=0 \text { (due to secular terms) }}=0
$$

Integrating once more we obtain

$$
\theta_{5}=\frac{\tilde{r}+\tilde{r}^{2}}{\omega_{0}^{3}}[\cos \theta(s)-1]+\frac{3}{16} \frac{\tilde{r}^{2}}{\omega_{0}^{3}}\left[\cos 2 \theta_{0}(s)-1\right] \text { and } \omega_{4}=-\frac{1}{2} \frac{\tilde{r}^{2}}{\omega_{0}^{3}}
$$

Defining $\Delta=\varepsilon / \omega_{0}$ and neglecting higher order terms, the expansion of $\theta(\tau)$ reads

$$
\begin{aligned}
\theta(\tau)=\tilde{\omega} \tau+\Delta^{2} \tilde{r} \sin \tilde{\omega} \tau+\Delta^{3} \tilde{r}(\cos \tilde{\omega} \tau-1)+\frac{1}{8} \Delta^{4} \tilde{r}^{2} \sin 2 \tilde{\omega} \tau-\Delta^{4} \tilde{r} \sin \tilde{\omega} \tau \\
+\frac{3}{16} \Delta^{5} \tilde{r}(\sin 2 \tilde{\omega} \tau-1)-\Delta^{5}\left(\tilde{r}+\tilde{r}^{2}\right)(\cos \tilde{\omega} \tau-1)+\cdots
\end{aligned}
$$

and

$$
\tilde{\omega}=\frac{\omega}{\lambda}-\frac{1}{2} \tilde{r} \Delta^{3} \varepsilon-\cdots .
$$

Now, we have everything set for the estimation of $\int_{0}^{\tilde{T}} \cos \theta(\tau) d \tau$. Discarding terms $\varepsilon^{n}$ with $n \geq 4$, we can express the $\cos \theta(\tau) d \tau$ in terms of Bessel functions as follows

$$
\begin{aligned}
\cos \theta(s) & \simeq \cos \left[\tilde{\omega} \tau+a \sin \left(\tilde{\omega} \tau+C+\Delta^{3} \tilde{r}\right)\right] \\
& =\cos (\tilde{\omega} \tau) \cos \left[a \sin \left(\tilde{\omega} \tau+C+\Delta^{3} \tilde{r}\right)\right]-\sin (\tilde{\omega} \tau) \sin \left[a \sin \left(\tilde{\omega} \tau+C+\Delta^{3} \tilde{r}\right)\right] \\
& =J_{0}(a) \cos (\tilde{\omega} \tau)-2 J_{1}(a) \sin (\tilde{\omega} \tau) \sin \left[\tilde{\omega} \tau+C+\Delta^{3} \tilde{r}\right]+\text { (higher harmonics) }
\end{aligned}
$$


where we used the relations

$$
\begin{aligned}
& \cos [x \sin \theta]=J_{0}(x)+2 \sum_{n=1}^{\infty} J_{2 n}(x) \cos (2 n \theta) \\
& \sin [x \sin \theta]=2 \sum_{n=1}^{\infty} J_{2 n-1}(x) \sin [(2 n-1) \theta]
\end{aligned}
$$

with $J_{n}(x)$ being the Bessel functions and

$$
\begin{gathered}
\Delta^{2} \tilde{r} \sin \tilde{\omega} \tau+\Delta^{3} \tilde{r} \cos \tilde{\omega} \tau=a \sin \left(C+\Delta^{3} \tilde{r}\right) \\
a=\Delta^{2} \tilde{r} \sqrt{1+\Delta^{2}}, \sin C=\frac{\Delta}{\sqrt{1+\Delta^{2}}}, \cos C=\frac{1}{\sqrt{1+\Delta^{2}}} .
\end{gathered}
$$

Integrating Eq. A.18 leads to

$$
\begin{aligned}
\int_{0}^{\tilde{T}} \cos \theta(\tau) d \tau & =-\int_{0}^{\tilde{T}} 2 J_{1}(a) \sin (\tilde{\omega} \tau) \sin \left[\tilde{\omega} \tau+C+\Delta^{3} \tilde{r}\right] d \tau \\
& =-2 J_{1}(a) \int_{0}^{\tilde{T}} \sin (\tilde{\omega} \tau)\left[\sin (\tilde{\omega} \tau) \cos \left(C+\Delta^{3} \tilde{r}\right)+\cos (\tilde{\omega} \tau) \sin \left(C+\Delta^{3} \tilde{r}\right)\right] d \tau \\
& =-\frac{2 \pi}{\tilde{\omega}} J_{1}(a) \cos \left(C+\Delta^{3} \tilde{r}\right)
\end{aligned}
$$

The analogous computation of $\int_{0}^{\tilde{T}} \sin \theta(\tau) d \tau$ yields

$$
\int_{0}^{\tilde{T}} \sin \theta(\tau) d \tau=\frac{2 \pi}{\tilde{\omega}} J_{1}(a) \sin \left(C+\Delta^{3} \tilde{r}\right)
$$

Since $\Delta \ll 1$, we can approximate $J_{1}(a) \simeq \frac{1}{2} a=\frac{1}{2} \Delta^{2} \tilde{r} \sqrt{1+\Delta^{2}}$, which together with the expansion of $\cos \left(\Delta^{3} \tilde{r}\right)$ and $\sin \left(\Delta^{3} \tilde{r}\right)$ gives

$$
\begin{aligned}
J_{1}(a) \cos \left(C+\Delta^{3} \tilde{r}\right) & \simeq \frac{1}{2} \Delta^{2} \tilde{r} \sqrt{1+\Delta^{2}}\left[\cos \left(\Delta^{3} \tilde{r}\right) \frac{1}{\sqrt{1+\Delta^{2}}}-\sin \left(\Delta^{3} \tilde{r}\right) \frac{\Delta}{\sqrt{1+\Delta^{2}}}\right] \\
& =\frac{1}{2} \Delta^{2} \tilde{r}\left[1-\frac{\left(\Delta^{3} \tilde{r}\right)^{2}}{2}+\ldots-\Delta\left(\Delta^{3} \tilde{r}-\frac{\left(\Delta^{3} \tilde{r}\right)^{3}}{6}+\ldots\right)\right] .
\end{aligned}
$$

Hence, neglecting terms of order $\Delta^{4}$ we obtain

$$
J_{1}(a) \cos \left(C+\Delta^{3} \tilde{r}\right) \simeq \frac{1}{2} \Delta^{2} \tilde{r} .
$$

Repeating the calculations for $J_{1}(a) \sin \left(C+\Delta^{3} \tilde{r}\right)$ :

$$
\begin{aligned}
J_{1}(a) \sin \left(C+\Delta^{3} \tilde{r}\right) & \simeq \frac{1}{2} \Delta^{2} \tilde{r} \sqrt{1+\Delta^{2}}\left[\cos \left(\Delta^{3} \tilde{r}\right) \frac{\Delta}{\sqrt{1+\Delta^{2}}}+\sin \left(\Delta^{3} \tilde{r}\right) \frac{1}{\sqrt{1+\Delta^{2}}}\right] \\
& =\frac{1}{2} \Delta^{2} \tilde{r}\left[\Delta\left(1-\frac{\left(\Delta^{3} \tilde{r}\right)^{2}}{2}+\ldots\right)+\left(\Delta^{3} \tilde{r}-\frac{\left(\Delta^{3} \tilde{r}\right)}{6}+\ldots\right)\right],
\end{aligned}
$$

which neglecting terms of order $\Delta^{4}$ leads to

$$
J_{1}(a) \sin \left(C+\Delta^{3} \tilde{r}\right) \simeq \frac{1}{2} \Delta^{3} \tilde{r} .
$$


Finally, with Eq. A.19, A.22, A.20, A.24 we arrive at the following estimations

$$
\begin{aligned}
\int_{0}^{\tilde{T}} \cos \theta(\tau) d \tau & =-\frac{\pi}{\tilde{\omega}} \Delta^{2} \tilde{r} \\
\int_{0}^{\tilde{T}} \sin \theta(\tau) d \tau & =\frac{\pi}{\tilde{\omega}} \Delta^{3} \tilde{r} .
\end{aligned}
$$

Recalling that $\delta=(\lambda m)^{-1}, \Delta=(m \omega)^{-1}, \tilde{r}=\lambda m R$, and substituting Eq. A.25 in A leads to the contribution to the order parameter due to drifting oscillators

$$
R_{\text {drift }}^{\mathrm{I}, \mathrm{II}}=-m \lambda R \int_{\omega_{\mathrm{P}, \mathrm{D}}} \frac{1}{(m \omega)^{2}} g(\omega) d \omega
$$

which is Eq. 2.46 of the main text.

After these steps, the calculation of the contribution due to drifting oscillators for the network case becomes straightforward; once the equations of motion are written in an equivalent form of Eq. A.2, the previous results related to the estimation of the running periodic solution can be simply extended by relating the parameters in the new problem with the ones above. To this end, let us consider Eq. 4.5 of the main text representing the dynamics of second-order Kuramoto oscillators in uncorrelated networks:

$$
\ddot{\phi}+\alpha \dot{\phi}+k \lambda r \sin \phi=D[k-\langle k\rangle-C(\lambda r)]
$$

Note that in the previous equation the equivalent coupling is proportional to $\lambda k$ and that the mass $m$ was set to $m=1$, while the damping factor $\alpha$ is considered as a free parameter in the model. These modifications with respect the original formulation make it convenient the adoption of the new time-scale $\tau=(k \lambda) t$ and, supposing again an underdamped regime, the introduction of the parameter $\varepsilon=\alpha / k \lambda \ll 1$. Thus, Eq. A.28 is transformed into

$$
\frac{d^{2} \phi}{d \tau^{2}}+\varepsilon \frac{d \phi}{d \tau}+\varepsilon^{2} \tilde{r} \sin \phi=\varepsilon \Gamma
$$

where $r / \alpha=\tilde{r} \varepsilon$ with $\tilde{r}$ being an arbitrary constant, and $\Gamma=D[k-\langle k\rangle-C(\lambda r)] / k \alpha \lambda$. Under these definitions, the constants $\Delta$ and $\tilde{r}$ assume the form $\Delta=\alpha^{2} /[D(k-\langle k\rangle-$ $C(\lambda r))]$ and $\tilde{r}=r k \lambda / \alpha^{2}$. Hence, the estimations for $\sin \phi(\tau)$ and $\cos \phi(\tau)$ become

$$
\begin{aligned}
\int_{0}^{\tilde{T}} \cos \phi(\tau) d \tau & =-\frac{\pi}{\tilde{\omega}} \frac{r k \lambda \alpha^{2}}{D^{2}[k-\langle k\rangle-C(\lambda r)]^{2}}, \\
\int_{0}^{\tilde{T}} \sin \phi(\tau) d \tau & =\frac{\pi}{\tilde{\omega}} \frac{r k \lambda \alpha^{4}}{D^{3}[k-\langle k\rangle-C(\lambda r)]^{3}} .
\end{aligned}
$$

Inserting Eq. A.30 into Eq. A.2 we then obtain the analytic approximation for the contribution of drifting oscillators in uncorrelated networks with natural frequencies proportional to degrees:

$$
r_{\mathrm{drift}}=\left(-\int_{k_{\min }}^{k_{1}}+\int_{k_{2}}^{k_{\max }}\right) \frac{-r k^{2} \lambda \alpha^{2} P(k)}{D^{2}[k-\langle k\rangle-C(\lambda r)]^{2}\langle k\rangle} d k .
$$


At last, substituting Eq. A.31 in Eq. 4.14 leads to the implicit equation for $C(\lambda r)$ :

$$
\begin{aligned}
0= & \frac{1}{\langle k\rangle} \int_{k_{1}}^{k_{2}} k P(k) \frac{D(k-\langle k\rangle-C(\lambda r))}{k \lambda r} d k \\
& +\frac{1}{2\langle k\rangle}\left(-\int_{k_{\min }}^{k_{1}}+\int_{k_{2}}^{k_{\max }}\right) \frac{r k^{2} \lambda \alpha^{4} P(k)}{D^{3}[k-\langle k\rangle-C(\lambda r)]^{3}} d k .
\end{aligned}
$$




\section{APPENDIX B - Additional diagrams for coupled Rössler oscillators}

\section{B.1 Incoherent traveling waves in the absence of coupling mis- match}

In Sec. 5.5.1 of the main text, it was shown that incoherent traveling waves emerge for large negative values of $K_{0} G_{0}$ in the presence of in- and out-coupling mismatches. However, this poses the question about whether such states are also attainable in the absence of coupling mismatches between the oscillators. Figure 32 shows the order parameter $r$ and the drift in the frequencies $\left|\Omega-\omega_{0}\right|$ of weighted $x$-coupled Rössler oscillators (Eq. 5.51) with phase-coherent and funnel attractors as function of $K_{0}$ and $G_{0}$ considering $\Delta K=\Delta G=0$. Again, as in the case where $\Delta K \neq 0, \Delta G \neq 0$, traveling waves are found in regions with sufficiently negative values of $K_{0} G_{0}$. Interestingly, this shows that the presence of a phase-lag between two populations is not a necessary condition for the emergence of a spontaneous drift different from the ensemble's mean frequency, contrary to what is observed in the collective dynamics of conformists and contrarians phase oscillators reported in Sec. 5.3. In fact, as shown there, not only the condition $\delta \neq 0$, but also asymmetric coupling is required for the existence of TWs in ensembles of phase oscillators. This suggests that asymmetric coupling is not a necessary condition neither for the observation of incoherent TWs. Note that for $\Delta K=\Delta G=0$ only a single coupling strength exists, namely $K_{0} G_{0}$. However, in order to better compare with the main text, we show the results of Fig. 32 in the $G_{0} \times K_{0}$ plane, similarly as in Figs. 30 and 31 .

\section{B.2 Dependence of Incoherent TWs on system size}

Another pertinent question in our study is the verification of whether incoherent TWs persist in larger populations. In order to address this issue, in Fig. 33 we report $\mid \Omega-$ $\omega_{0} \mid$ and $r$ as function of the system size $N$ considering $\left(G_{0}, K_{0}\right)=(-0.5,0.5)$, parameters for which extreme values of $\left|\Omega-\omega_{0}\right|$ are observed in Fig. 30. Interestingly, no sensitive dependence on the number of oscillators is found for the magnitude of the TWs, showing that the phenomenon is indeed not a consequence of finite-size fluctuations. 

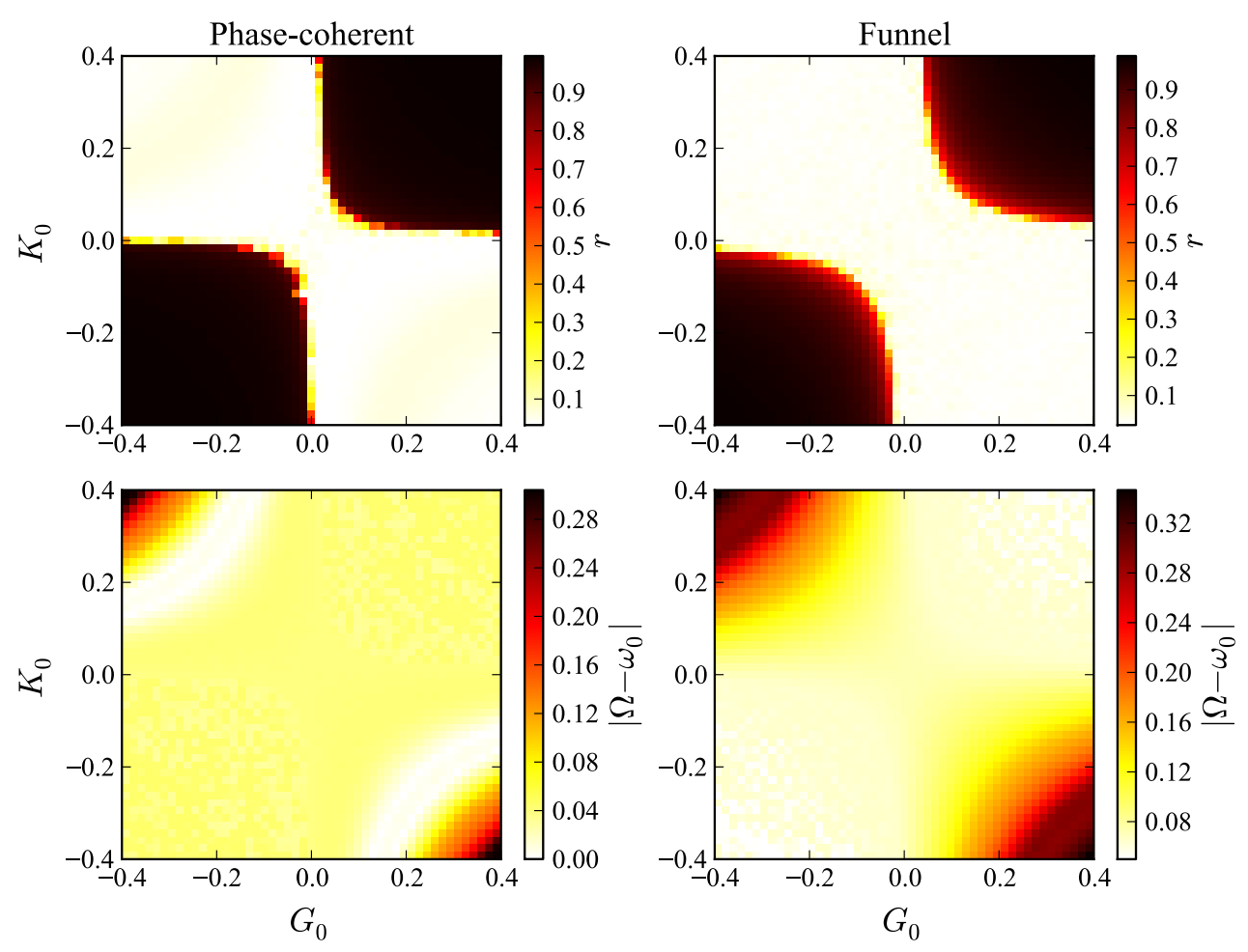

Figure 32 - Colormaps for weighted $x$-coupled Rössler oscillators with phase-coherent $(a=0.15$, $b=0.2$ and $c=10)$ and funnel attractors $(a=0.2, b=0.2$ and $c=7)$ in the absence of coupling mismatches, i.e. $\Delta K=0, \Delta G=0$. Parameters: $N=10^{3}, d t=0.05$. For each $\left(G_{0}, K_{0}\right)$ in the $50 \times 50$ grid, the quantities are averaged over $\mathrm{t} \in[4700,5000]$. Initial conditions $\left(x_{i}(0), y_{i}(0), z_{i}(0)\right)$ are selected from an uniform distribution in the range $[-1,1]$.

Source: Adapted from PERON et al. (161)

\section{B.3 Evolution of order parameters}

In this section we report on the relaxation dynamics of weighted $x$-coupled Rössler oscillators. Figure 34 shows typical evolutions of the order parameters observed in lag sync and $\pi$-states. Similar results are obtained for zero-lag sync and incoherent states as well as for phase-coupled oscillators.

\section{B.4 Local order parameters}

In order to also characterize the dynamics within the subpopulations, in this section we present diagrams for the local order parameters $r_{1,2}$ for weighted $x$-coupled and phase-coupled models, shown in Fig. 35 and 36, respectively. We repeat the respective maps for variables $r, \delta$ and $\Omega$ shown in Figs. 30 and 31. Noteworthy, we remark that one does not need to rely on parameters $r_{1}$ and $r_{2}$ when detecting TWs. The latter states 


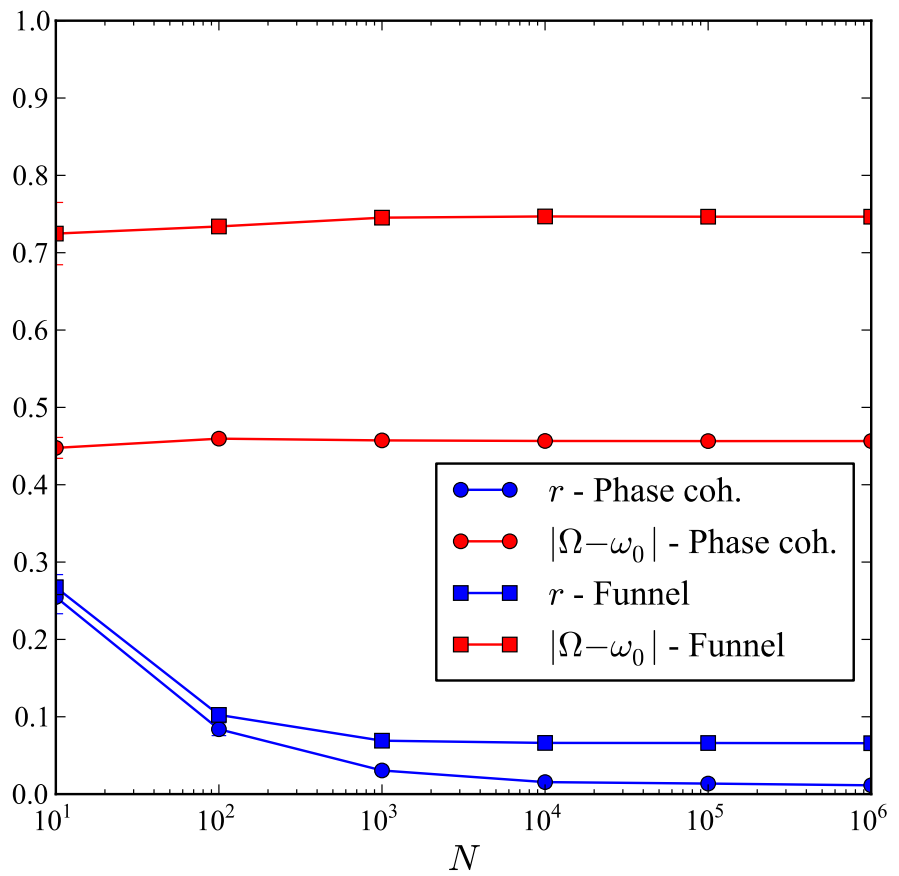

Figure 33 - Dependence of incoherent TWs on the system size for $K_{0}=0.5, G_{0}=-0.5, \Delta K=$ $0.1, \Delta G=1$ and $D=0.03$. Each point is an average over 100 different realizations. Size of errorbars is smaller than the dots' sizes.

Source: Adapted from PERON et al. (161)

are solely described by the emergence of a new frequency different from the natural one, i.e. when the condition $\left|\Omega-\omega_{0}\right|>0$ is fulfilled. The consideration of other parameters is necessary only when distinguishing incoherent from coherent TWs.(161)

\section{B.5 Additional diagrams for the phase-coupled model}

Figure 37 shows zoomed colormaps around the TW regions of Fig. 31 related to the phase-coupled model (Eq. 5.57). Precisely, Fig. 37 (a) depicts the TW state surrounded by the $\pi$-state region, whereas Fig. 37 (b) highlights the TW state reached via the zerolag state. We recall that the emergence of TW and $\pi$-states as observed in Fig. 37 in the phase-coupled Rössler model bears a striking similarity with the stochastic Kuramoto model in Chapter 5, despite the fact that in the former the phases evolution explicitly depend on the chaotic amplitudes. 

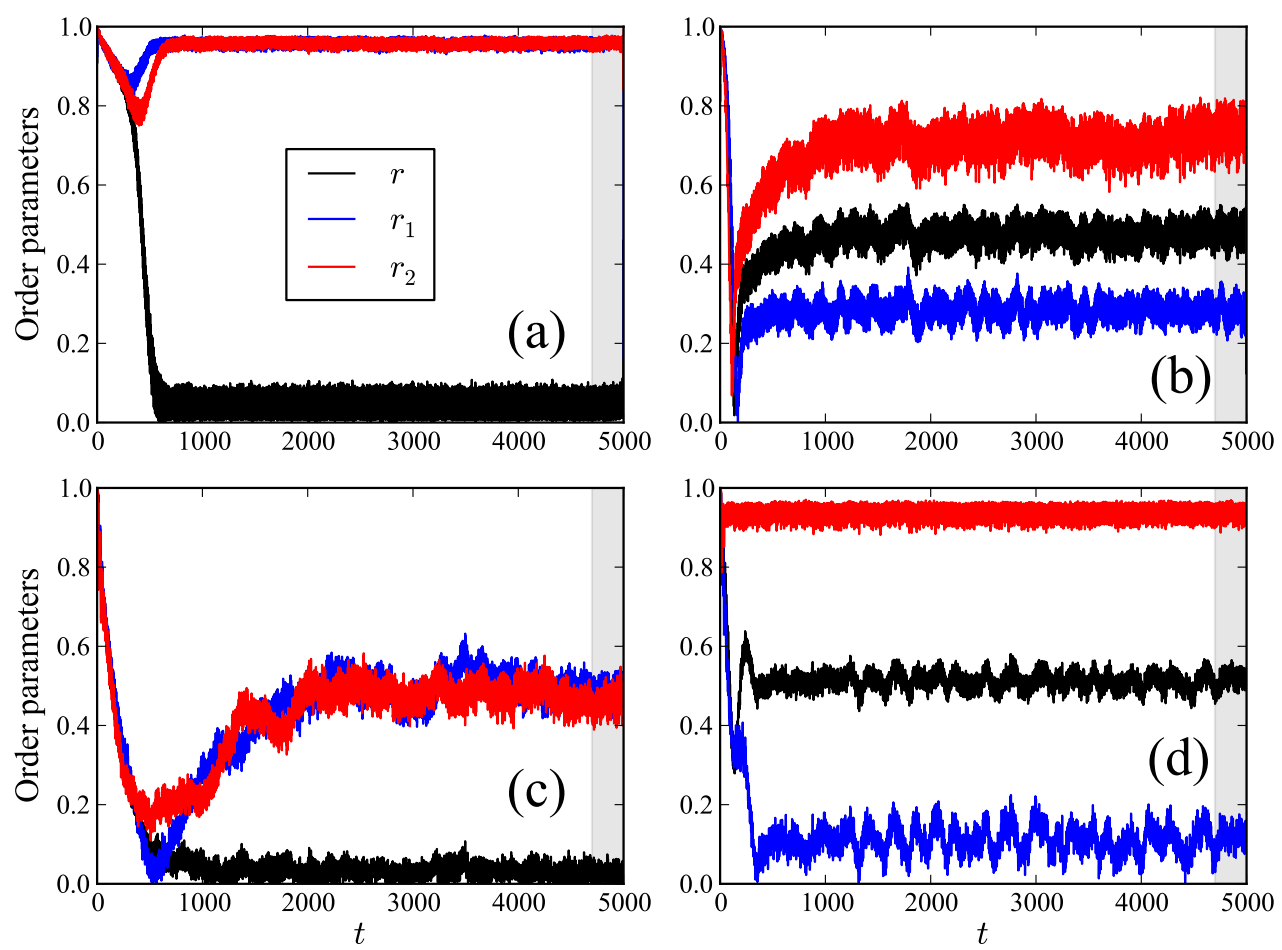

Figure 34 - Examples of time evolution of order parameters for phase-coherent oscillators in (a) $\pi$-state $\left(K_{0}=G_{0}=0\right)$, (b) lag sync $\left(K_{0}=0.25, G_{0}=-0.1\right)$; and oscillators with funnel attractor also in (c) $\pi$-state $\left(K_{0}=G_{0}=0\right)$ and (d) lag sync $\left(K_{0}=0.03\right.$, $\left.G_{0}=0.5\right)$. Other parameters: $\Delta K=0.1, \Delta G=1, D=0.03$ and $N=10^{3}$. Shaded area corresponds to the time averaging window considered in Fig. 30.

Source: Adapted from PERON et al. (161)

\section{B.6 Comparison between locking and mean ensemble frequencies}

Traveling phase waves are generally detectable either via the mean ensemble frequency $\Omega$ (Eq. 5.55) or in terms of the locking frequency $\dot{\psi}$ (see also the discussion at the end of Sec. 5.3). Here we compare the performance of both measures when evaluating the TW regions for the phase-coupled Rössler model. Figure 38(a) reproduces the colormap of Fig. 31(c), while Fig. 38(b) shows the collective drifts in the frequency measured by $\left|\langle\dot{\psi}\rangle_{t}-\omega_{0}\right|$. As it is seen, the same patterns are uncovered with the particular difference that higher values for the latter measure are obtained. This fact is expected, since only oscillators synchronized with the mean-field contribute in the sum of Eq. 5.55. In Fig. 39 we compare in more detail the measures considering a small region of the parameter space in Fig. 38 for fixed $G_{0}$. As shown, the onset of the TW region is equally captured by both definitions. Finally, for the sake of comparison between coherent and incoherent TWs, Fig. 40 depicts, for fixed $K_{0}$, the variation of $\left|\Omega-\omega_{0}\right|$ in regions of incoherence and zero-lag synchronization considering oscillators with funnel attractors. 

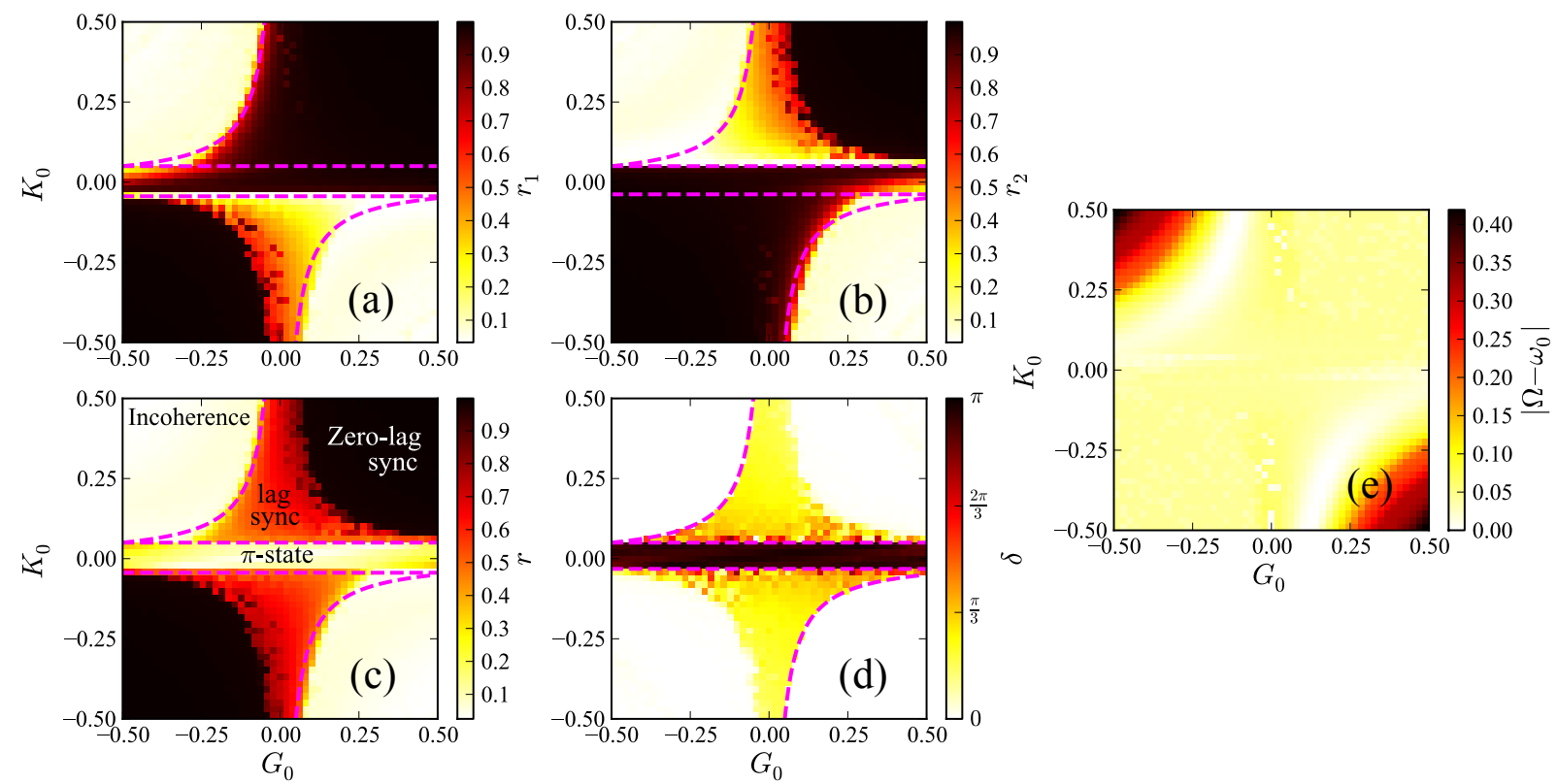

Figure 35 - Colormaps for weighted $x$-coupled Rössler oscillators with phase-coherent attractor ( $a=0.15, b=0.2$ and $c=10$ ). (a) $r_{1}$, (b) $r_{2}$, (c) $r$, (d) $\left|\Omega-\omega_{0}\right|$ and (e) $\delta$. Remaining parameters: $\Delta K=0.1, \Delta G=1, D=0.03$ and $N=10^{3}$. Notice in panel (d) that the white upper left and lower right regions are undefined since the phase lag is undefined. On the other hand, the frequency difference [(e)] defined via the Hilbert transform (Eq. 5.55) indicates a strict acceleration.

Source: Adapted from PERON et al. (161)
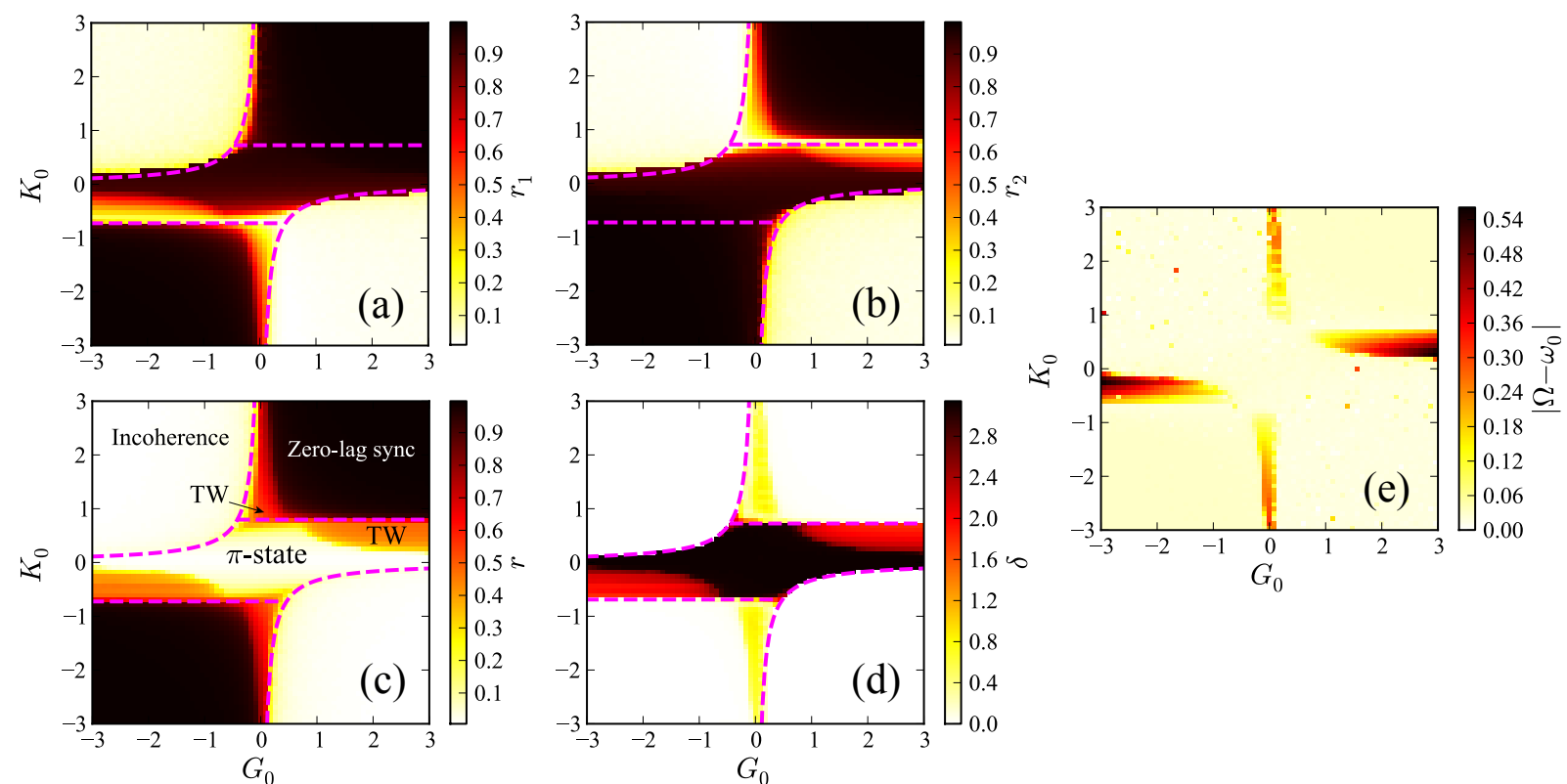

Figure 36 - Colormaps for phase-coupled Rössler oscillators with phase-coherent attractor $(a=$ $0.15, b=0.2$ and $c=10)$ for $\Delta K=1.45, \Delta G=1, D=0.03$ and $N=10^{3}$. Concerning (d) see the remark on the phase lag in Fig 35.

Source: Adapted from PERON et al. (161) 

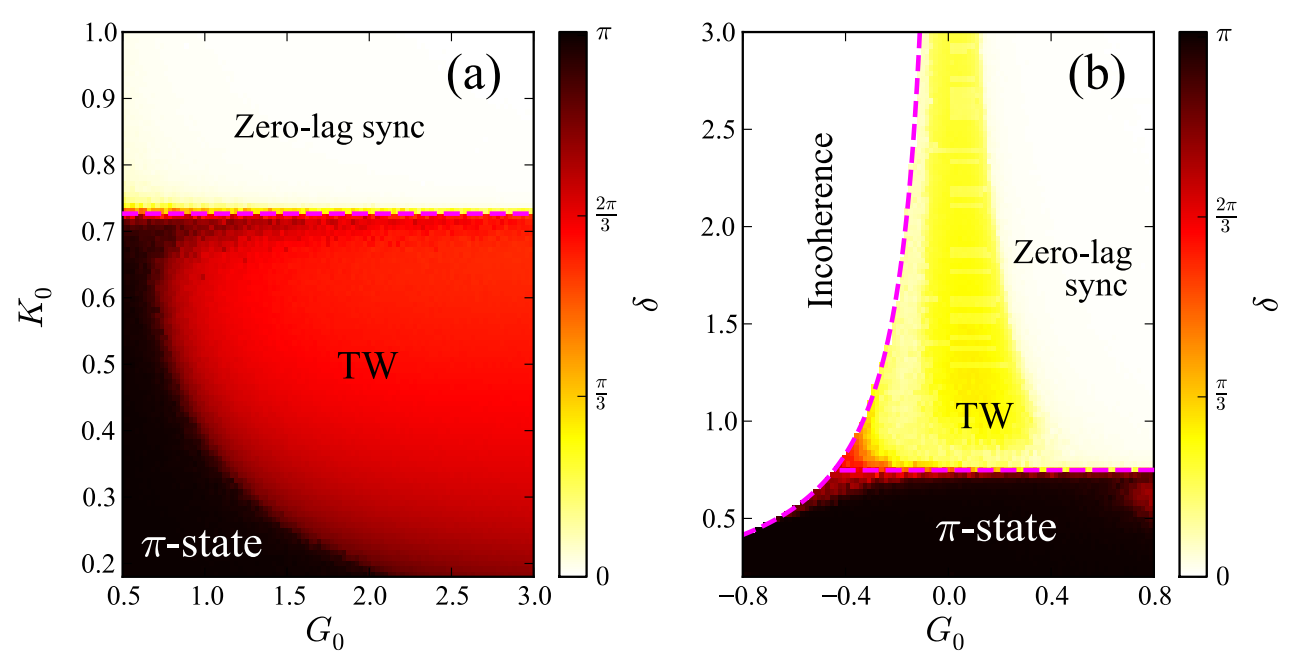

Figure 37 - The absolute value of the difference between the mean phases of the two populations, $\delta$, in dependence on the mean in- and out-coupling strengths, $K_{0}$ and $G_{0}$, respectively. The two panels show zoomed regions from Fig. 31, with different transition scenarios into the TW states. In (a) the TW region is surrounded by zero-lag states, whereas in (b) the TW states are surrounded by $\pi$-states. Parameters: $N=10^{3}$, $\Delta K=1.45, \Delta G=1, D=0.03, d t=0.05$. For each $\left(G_{0}, K_{0}\right)$ in the $100 \times 100$ grid, the quantities averaged over $t \in[4000,5000]$. Dashed magenta line in panel (a) marking the interface between Incoherence and Zero-lag sync is obtained by the critical condition $2 D=\langle\langle K G\rangle\rangle$, where $\langle\langle\ldots\rangle\rangle=\int d K^{\prime} \int d G^{\prime} \ldots P\left(K^{\prime}, G^{\prime}\right)$ and $P(K, G)$ is the joint probability distribution of in- and out-couplings. Horizontal dashed lines in both panels correspond to the critical points $K_{0 c}= \pm \Delta K / 2$.

Source: Adapted from PERON et al. (161)
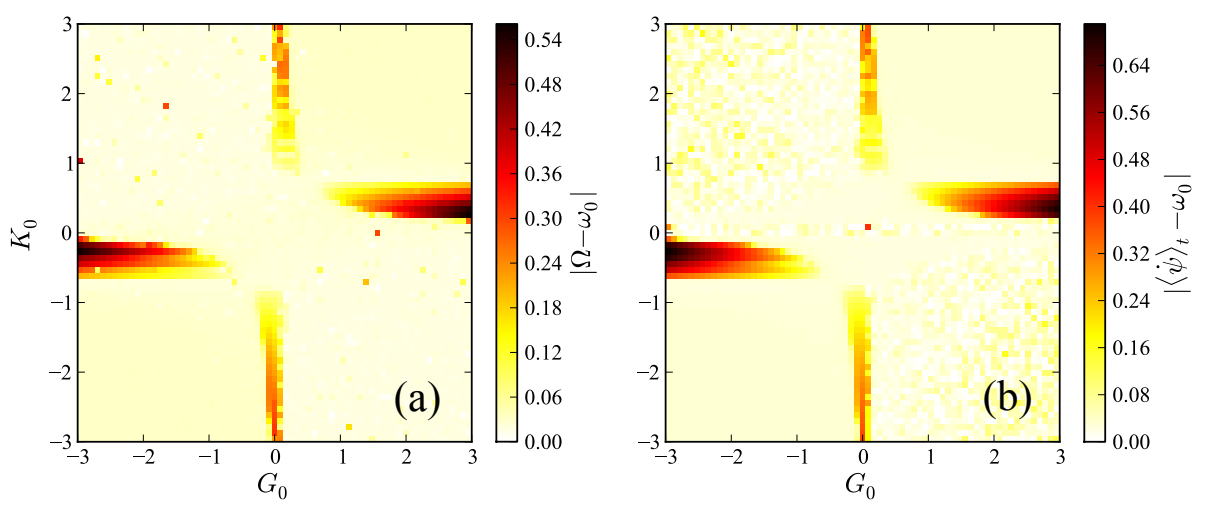

Figure 38 - Colormaps for (a) $\left|\Omega-\omega_{0}\right|$ and (b) $\left|\langle\dot{\psi}\rangle_{t}-\omega_{0}\right|$ of phase-coupled Rössler oscillators. Parameters: $N=10^{3}, \Delta K=1.45, \Delta G=1, D=0.03$, averaging over $t \in[4000,5000]$ and $d t=0.05$.

Source: Adapted from PERON et al. (161) 


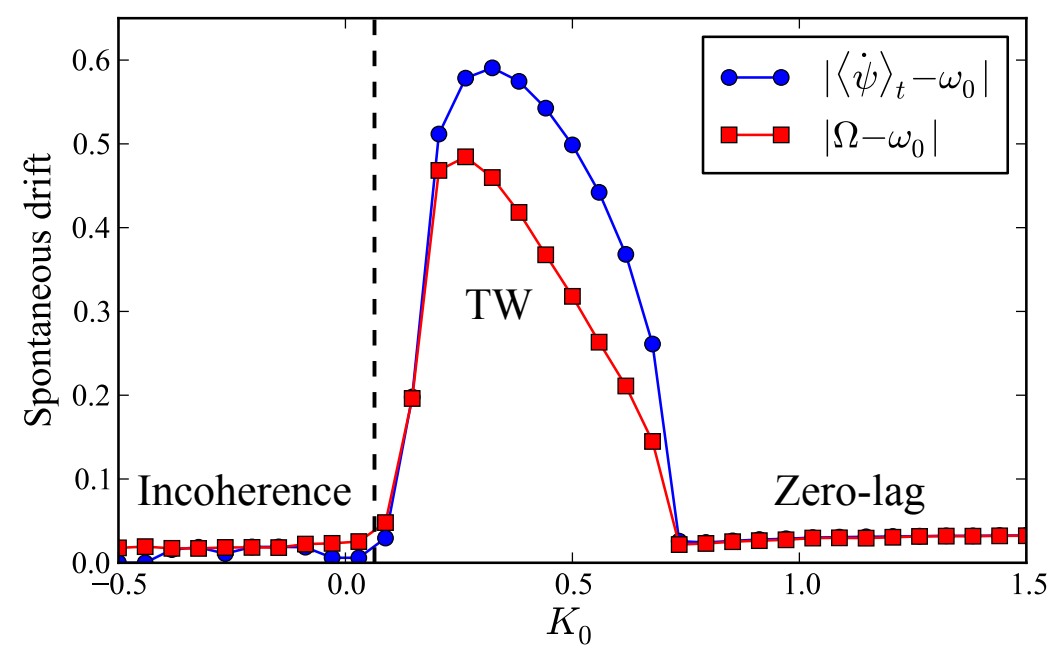

Figure 39 - Spontaneous drift measured by locking frequency $\langle\dot{\psi}\rangle_{t}$ and mean ensemble frequency $\Omega$. Parameters: $N=10^{4}, \Delta K=1.45, \Delta G=1, G_{0}=2.5, D=0.03$, averaging over $t \in[4000,5000]$ and $d t=0.05$.

Source: Adapted from PERON et al. (161)

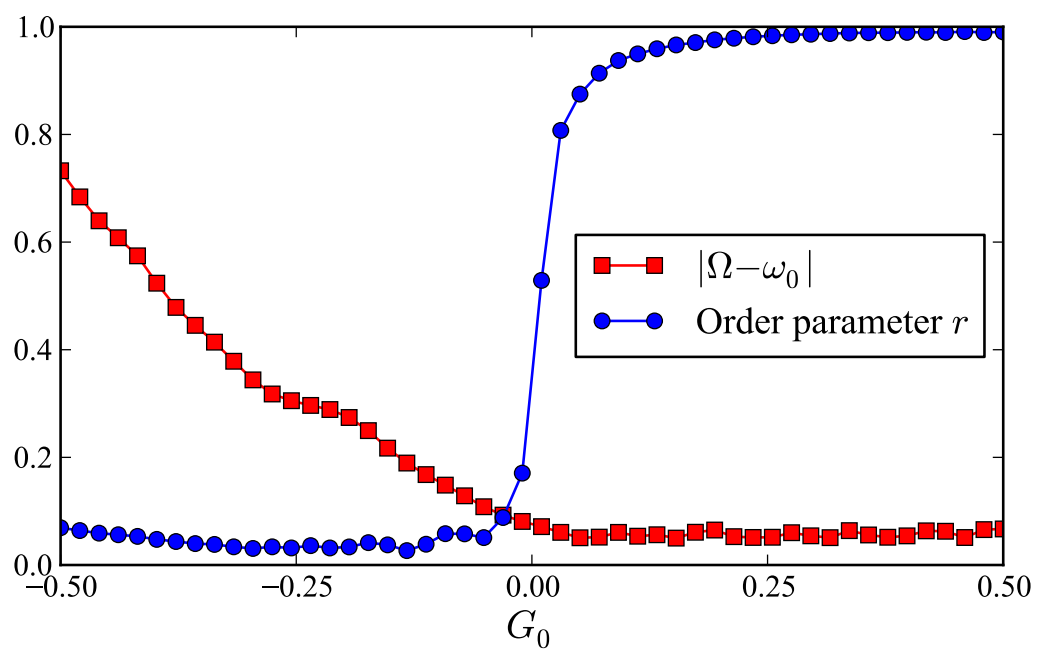

Figure 40 - Order parameter $r$ and mean ensemble frequency $\Omega$ as functions of $G_{0}$ in regions of incoherence and zero-lag synchronization for oscillators with funnel attractors. Parameters: $N=10^{3}, \Delta K=0.1, \Delta G=1, K_{0}=0.5$ and $D=0.03$.

Source: Adapted from PERON et al. (161) 



\section{APPENDIX C - List of publications}

This thesis is partially based on the following publications:

Thomas K. DM. Peron, Francisco A. Rodrigues, Jürgen Kurths. Synchronization of clustered random networks. Physical Review E, 87(3):032807, 2013.

Peng Ji, Thomas K. DM. Peron, Peter J. Menck, Francisco A. Rodrigues, and Jürgen Kurths. Cluster explosive synchronization in complex networks. Physical Review Letters, 110(21):218701, 2013.

Peng Ji, Thomas K. DM. Peron, Francisco A. Rodrigues, and Jürgen Kurths. Analysis of cluster explosive synchronization in complex networks. Physical Review E, 90(6):062810, 2014 .

Thomas K. DM. Peron, Peng Ji, Francisco A. Rodrigues, and Jürgen Kurths. Effects of assortative mixing in the second-order Kuramoto model. Physical Review E, 91(5):052805, 2015 .

Bernard Sonnenschein, Thomas K. DM. Peron, Francisco A. Rodrigues, Lutz SchimanskyGeier. Collective dynamics in two populations of noisy oscillators with asymmetric interactions. Physical Review E, 91(6):062910, 2015.

Thomas K. DM. Peron, Jürgen Kurths, Francisco A. Rodrigues, Lutz Schimansky-Geier, and Bernard Sonnenschein. Traveling phase waves in asymmetric networks of noisy chaotic attractors. Physical Review E, 94(4):042210, 2016.

Francisco A. Rodrigues, Thomas K. DM. Peron, Peng Ji, Jürgen Kurths. The Kuramoto model in complex networks. Physics Reports, 610:1-98, 2016.

During the course of this thesis we also had the opportunity to study other topics that we decide to not contemplate in the present text. Part of this work appeared in the following publications:

Guilherme F. de Arruda, Thomas K. DM. Peron, Marinho G. de Andrade, Jorge A. Achcar, Francisco A. Rodrigues. The influence of network properties on the synchronization of Kuramoto oscillators quantified by a Bayesian regression analysis, Journal of Statistical Physics, 152(3): 519-533, 2013.

Thomas K. DM. Peron, Cesar Comin, Diego Amancio, Luciano da F. Costa, Francisco A. Rodrigues, and Jürgen Kurths. Correlations Between Climate Network and Relief Data, Nonlinear Processes in Geophysics, 21: 1127-1132, 2014. 
Peng Ji, Thomas K.DM. Peron, Francisco A. Rodrigues, Jürgen Kurths. Low-dimensional behavior of Kuramoto model with inertia in complex networks, Scientific Reports, 4:4783, 2014 .

Bernard Sonnenschein, Thomas K. DM. Peron, Francisco A. Rodrigues, Jürgen Kurths, and Lutz Schimansky-Geier. Cooperative behavior between oscillatory and excitable units: the peculiar role of positive coupling-frequency correlations. European Physical Journal $B, 87: 182,2014$.

Deniz Eroglu, Thomas K. DM. Peron, Norbert Marwan, Francisco A. Rodrigues, Luciano da F. Costa, Michael Sebek, Istvan Kiss, and Jürgen Kurths. Entropy of weighted recurrence plots. Physical Review E, 90(4):042919, 2014.

J. A. Méndez-Bermúdez,A. Alcazar-López, A. J. Martínez-Mendoza, Francisco A. Rodrigues and Thomas K. DM. Peron. Universality in the spectral and eigenfunction properties of random networks. Physical Review E, 91(3):032122, 2015.

Cheng Ye, Richard C. Wilson, Cesar Comin, Thomas K. DM. Peron, Filipi N. Silva, Francisco A. Rodrigues, Luciano da F. Costa, Andrea Torsello, and Edwin R. Hancock. Thermodynamic characterization of networks using graph polynomials. Physical Review E, 92(3):032810, 2015.

Filipi N. Silva, Cesar Comin, Thomas K. DM. Peron, Francisco A. Rodrigues, Cheng Ye, Richard C. Wilson, Edwin Hancock, Luciano da F. Costa. Concentric network symmetry, Information Sciences, 333:61-80, 2016.

Paul Schultz, Thomas Peron, Deniz Eroglu, Thomas Stemler, G. Marcelo R. Ávila, Francisco A. Rodrigues, Jürgen Kurths. Tweaking synchronization by connectivity modifications. Physical Review E, 93(6):062211, 2016. 